Aus der Poliklinik für Zahnärztliche Prothetik

(Prof. Dr. med. dent. R. Bürgers)

im Zentrum Zahn-, Mund- und Kieferheilkunde

der Medizinischen Fakultät der Universität Göttingen

\title{
Tageszeitabhängige Abweichungen der statischen und dynamischen Okklusion
}

\author{
INAUGURAL-DISSERTATION \\ zur Erlangung des Doktorgrades \\ der Zahnmedizin \\ der Medizinischen Fakultät der \\ Georg-August-Universität zu Göttingen \\ vorgelegt von
}

Bernhard Gerd Christian Wiechens

aus

Kassel

Göttingen 2020 
Dekan:

\section{Betreuungsausschuss}

Betreuer/in

Ko-Betreuer/in:

\section{Prüfungskommission}

Referent/in

Ko-Referent/in:

Drittreferent/in:

Datum der mündlichen Prüfung:
Prof. Dr. med. W. Brück

Prof. Dr. med. dent. R. Bürgers

Prof. Dr. med. dent. P. Meyer-Marcotty

Prof. Dr. med. dent. R. Bürgers

Prof. Dr. med. dent. P. Meyer-Marcotty

Prof. Dr. med. M. Oppermann 
Hiermit erkläre ich, die Dissertation mit dem Titel "Tageszeitabhängige Abweichungen der statischen und dynamischen Okklusion" eigenständig angefertigt und keine anderen als die von mir angegebenen Quellen und Hilfsmittel verwendet zu haben.

Göttingen, den

(Unterschrift) 


\section{Inhaltsverzeichnis}

$\underline{\text { Seite }}$

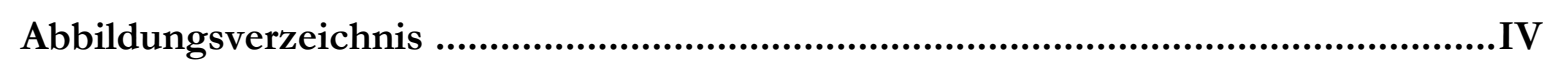

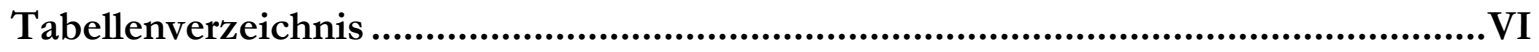

Abkürzungsverzeichnis............................................................................................... VIII

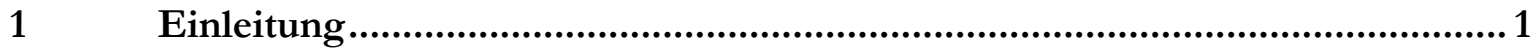

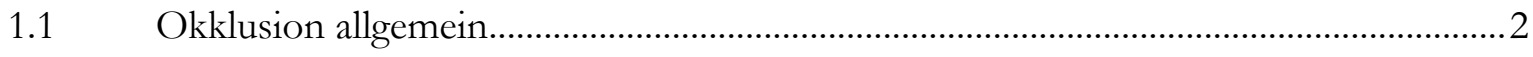

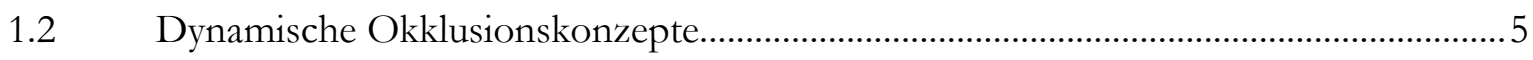

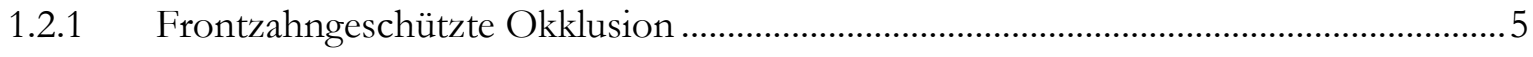

1.2.2 Eckzahngeschützte Okklusion …………..................................................................

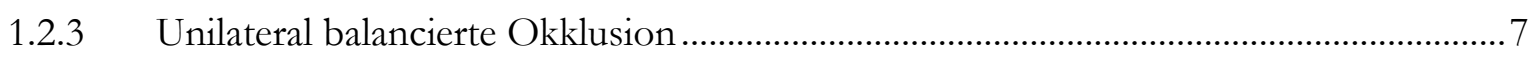

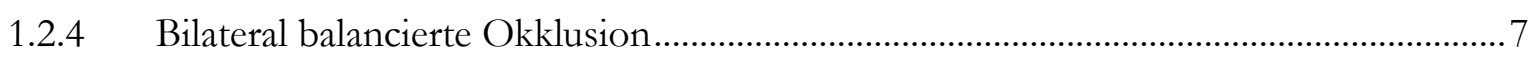

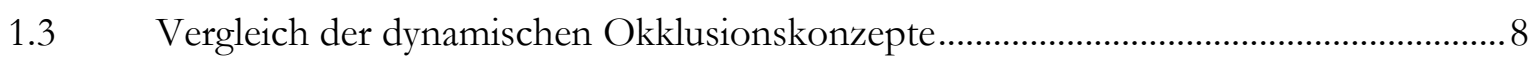

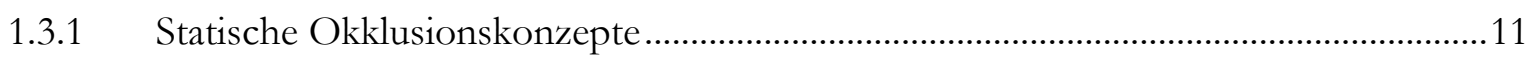

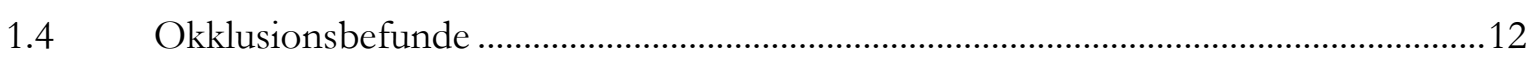

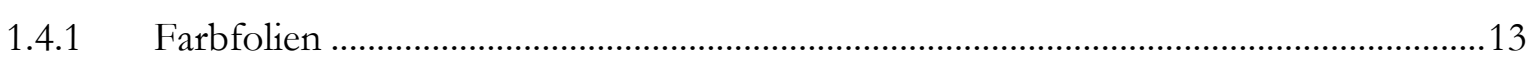

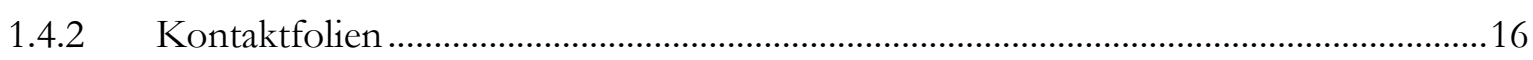

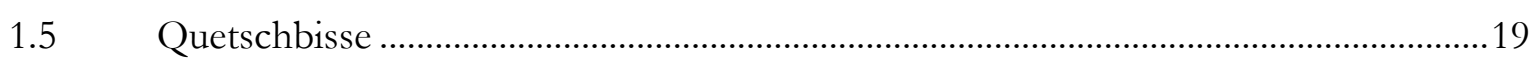

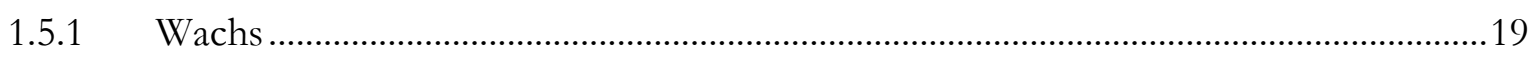

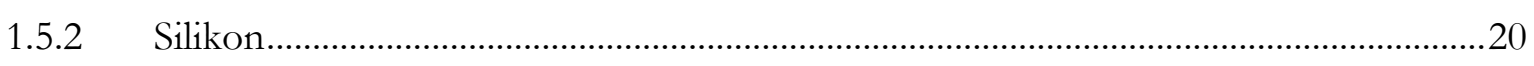

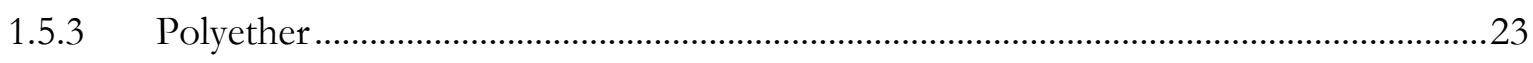

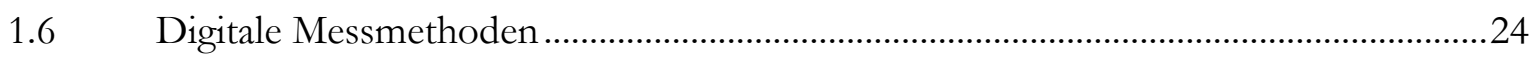

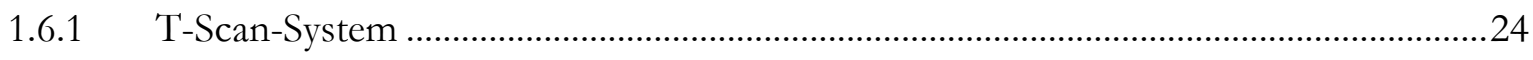

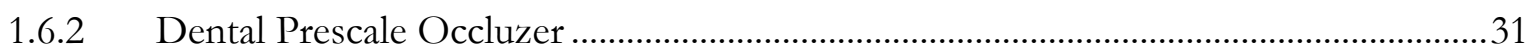

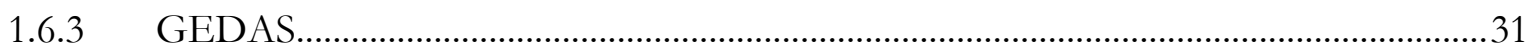

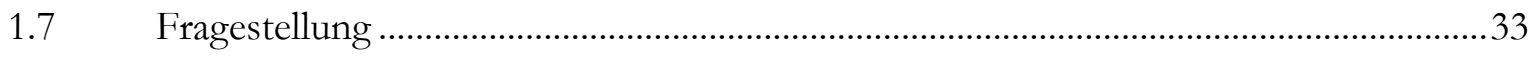

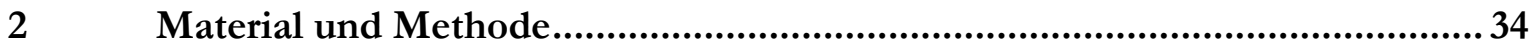

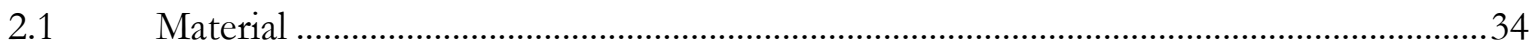

2.1.1 Digitale Okklusionsdiagnostik ........................................................................................

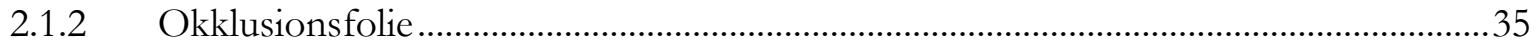

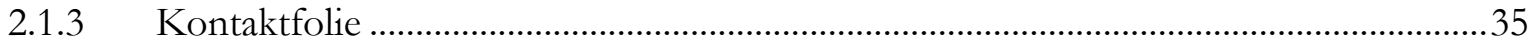

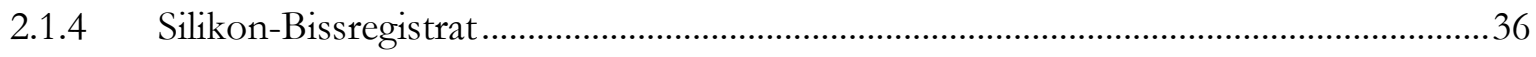

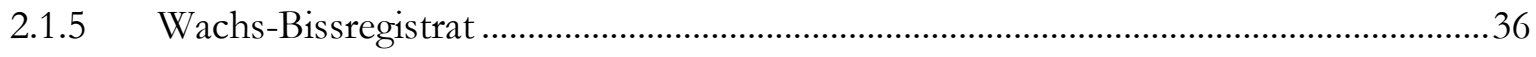

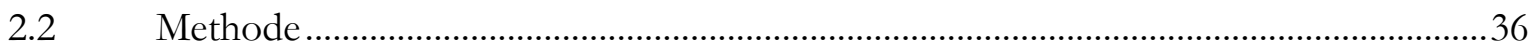




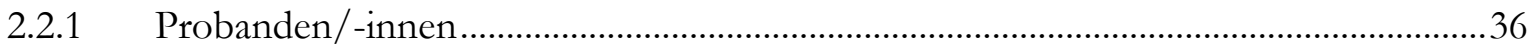

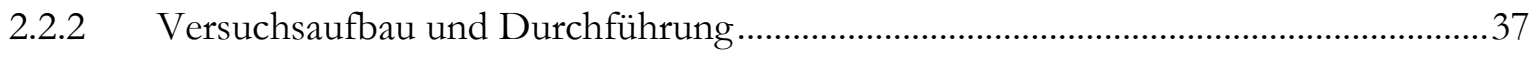

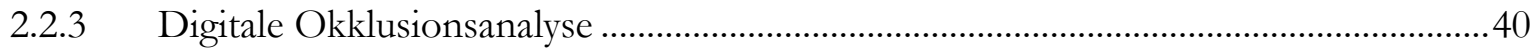

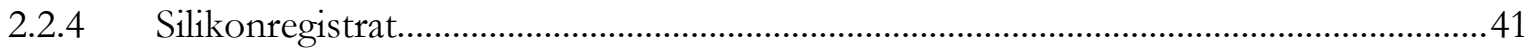

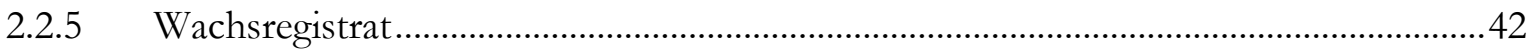

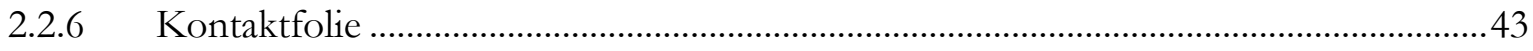

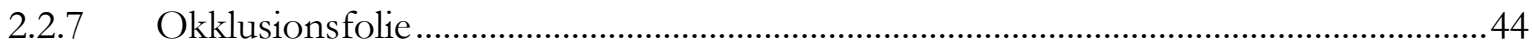

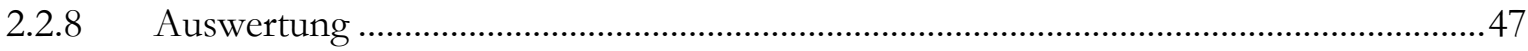

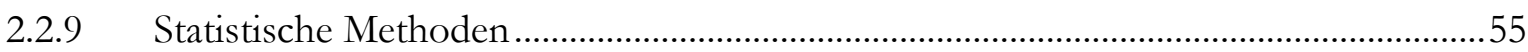

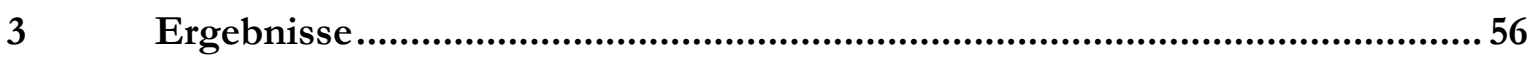

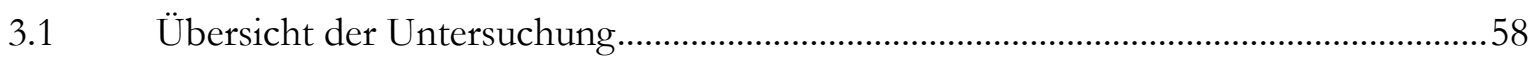

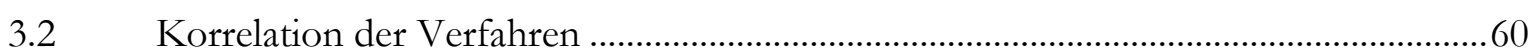

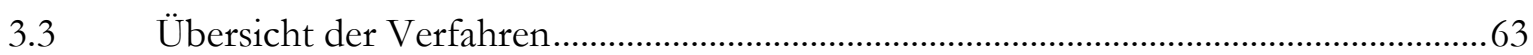

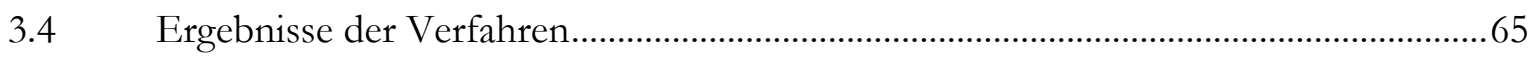

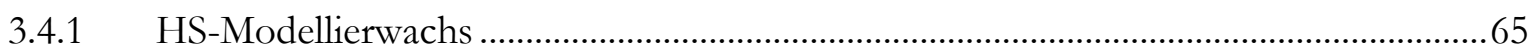

3.4.2 Okklusionsfolie $12 \mu \mathrm{m}$ zur Erhebung der statischen Okklusion ..................................66

3.4.3 Okklusionsfolie $12 \mu \mathrm{m}$ zur Erhebung der dynamischen Okklusion nach links..........67

3.4.4 Okklusionsfolie $12 \mu \mathrm{m}$ zur Erhebung der dynamischen Okklusion nach rechts .......68

3.4.5 Silikonregistrierung Registrado X-tra zur Erhebung der statischen Okklusion ..........69

3.4.6 Kontaktfolie Hanel Shimstock-Folie $8 \mu \mathrm{m}$ zur Erhebung der statischen

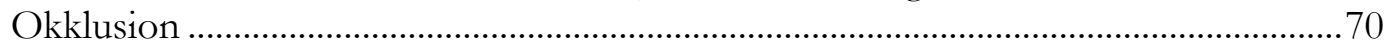

3.4.7 T-Scan-III-System zur Erhebung der statischen Okklusion .......................................... 70

3.4.8 T-Scan-III-System zur Erhebung der dynamischen Okklusion nach links ..................71

3.4.9 T-Scan-III-System zur Erhebung der dynamischen Okklusion nach rechts................72

3.5 Übersicht der wahrscheinlichen Veränderungen nach Verfahren....................................73

3.5.1 Wahrscheinlichkeiten für Veränderungen in der Okklusionsmessung pro Kiefer .....75

3.5.2 Einfluss der Tageszeit auf die Wahrscheinlichkeit eine Veränderung zu messen........76

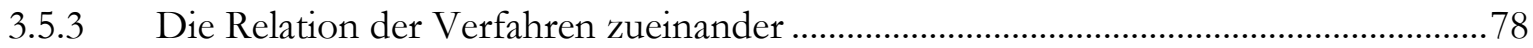

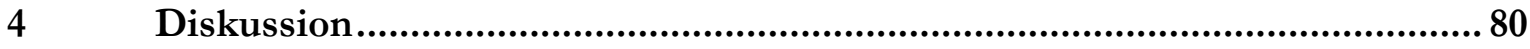

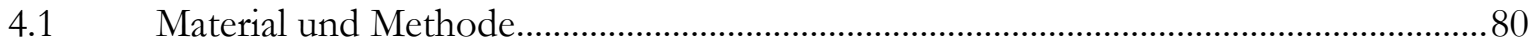

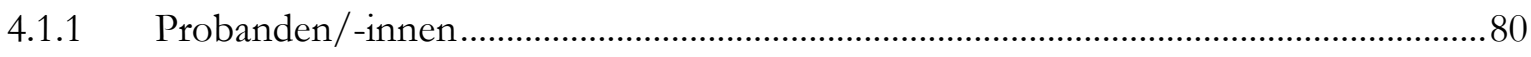

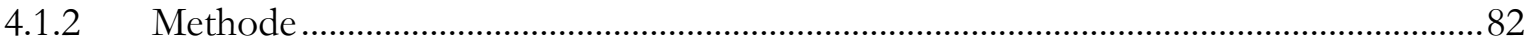

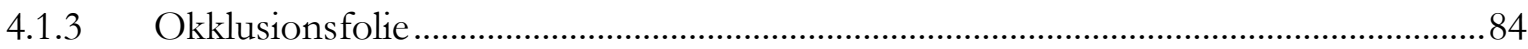

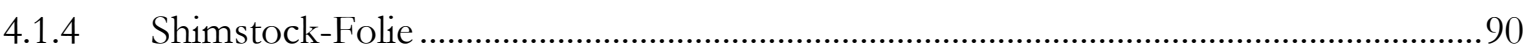

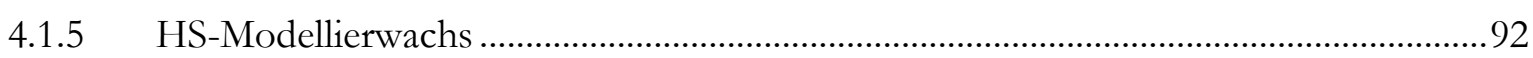

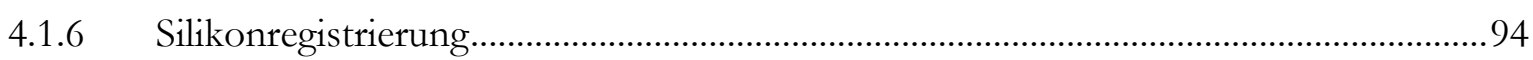




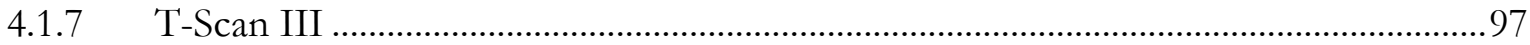

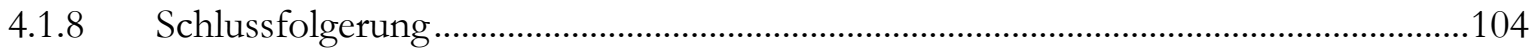

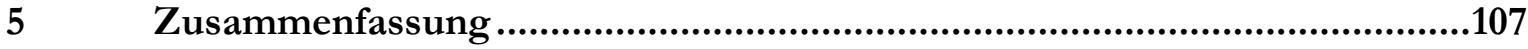

$6 \quad$ Anhang

6.1 Deskriptive Werte der erhobenen Daten.....................................................................109

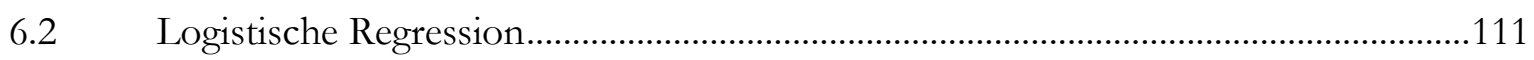

6.3 Logistische Regression ohne Tageszeitinteraktion ......................................................114

6.4 Logistische Regression zur Okklusionsänderung pro Kiefer.....................................115

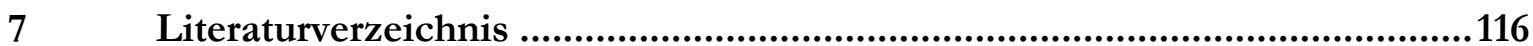




\section{Abbildungsverzeichnis}

$\underline{\text { Seite }}$

Abbildung 1: Frontzahngeschützte Okklusion. ........................................................................

Abbildung 2: Eckzahngeschützte Okklusion. ……..................................................................6

Abbildung 3: Unilateral balancierte Okklusion in vivo...................................................................

Abbildung 4: Unilateral balancierte Okklusion. ..............................................................................

Abbildung 5: Bilateral balancierte Okklusion in vivo ....................................................................

Abbildung 6: Bilateral balancierte Okklusion............................................................................. 8

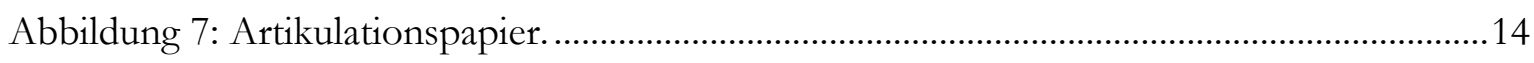

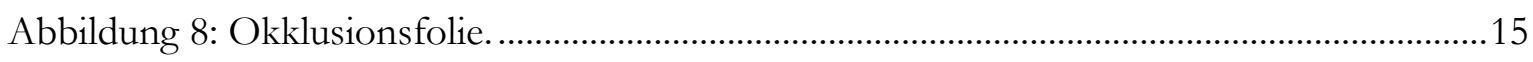

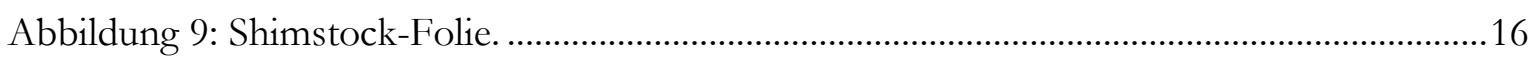

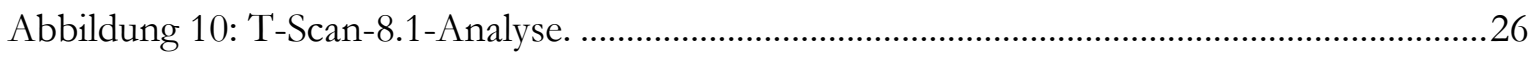

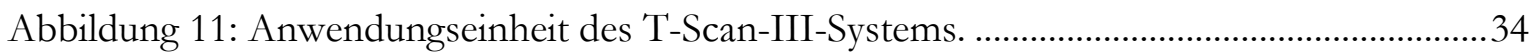

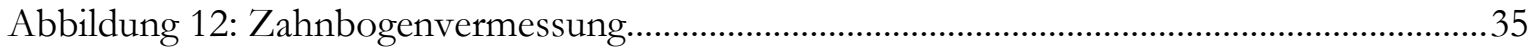

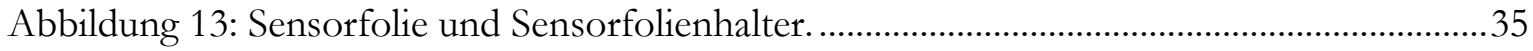

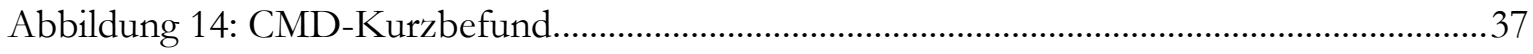

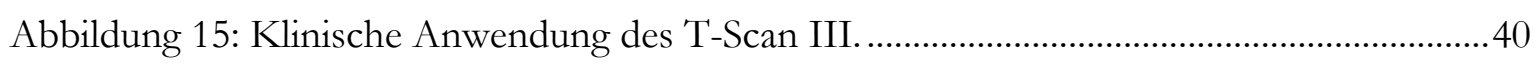

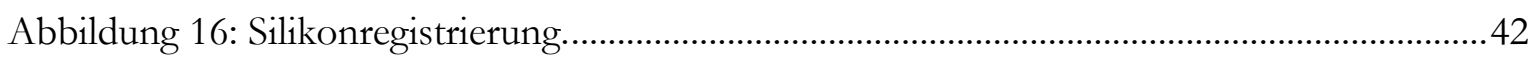

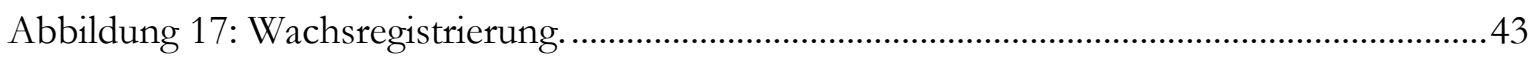

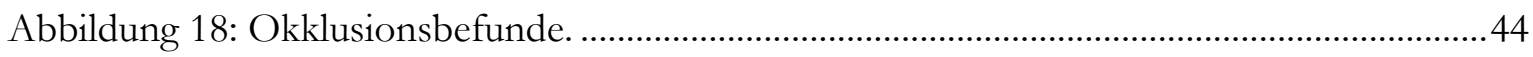

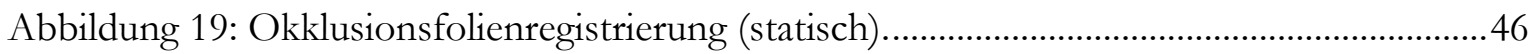

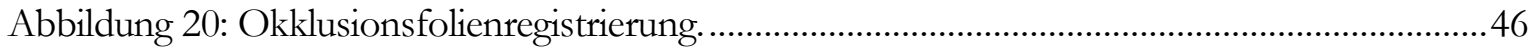

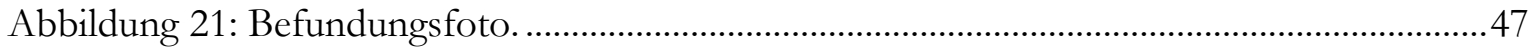

Abbildung 22: T-Scan-III-Analyseoberfläche der statischen Okklusion.......................................48

Abbildung 23: T-Scan-III-Analyseoberfläche der dynamischen Okklusion nach links.............49

Abbildung 24: T-Scan-III-Analyseoberfläche der hyperbalancierten Okklusion..........................49

Abbildung 25: Justierung der T-Scan-III-Analyseoberfläche........................................................ 50

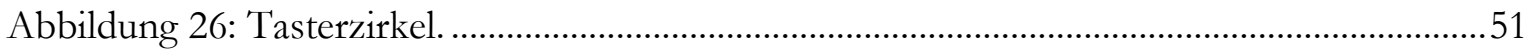


Abbildung 27: Auswertung der Silikonregistrierung. ........................................................................52

Abbildung 28: Auswertung der Wachsregistrierung..........................................................................53

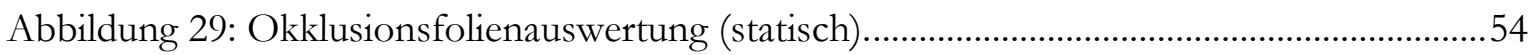

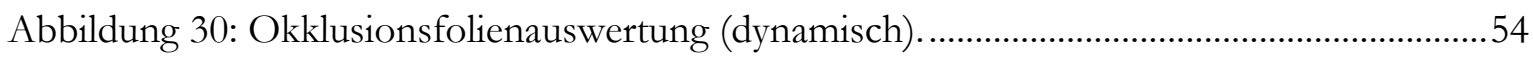

Abbildung 31: Paarweise Korrelation der verschiedenen Okklusionsregistrierungen. ................60

Abbildung 32: Hierarchisches Clustering der Okklusionsregistrierungen nach Korrelation....62

Abbildung 33: Erkannte Okklusionsveränderungen nach Messverfahren und Zahn.................63

Abbildung 34: Änderungsausmaß nach Messverfahren und Zahn............................................65 


\section{Tabellenverzeichnis}

$\underline{\text { Seite }}$

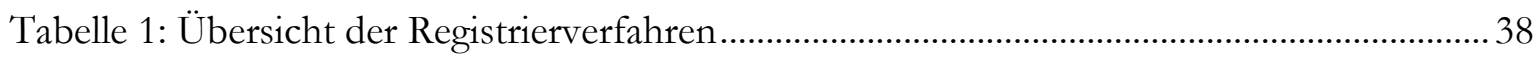

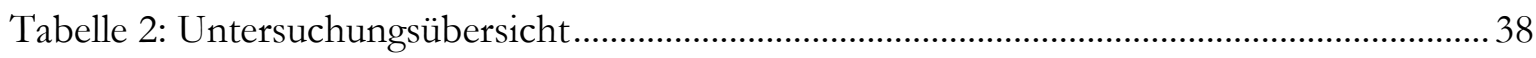

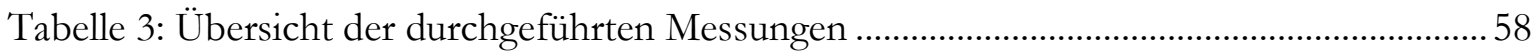

Tabelle 4: Messwertverteilung je Quadrant ....................................................................................... 59

Tabelle 5: Ermittelte Kontaktpunktanzahl je Zahn mittels Wachsregistrierung ............................ 66

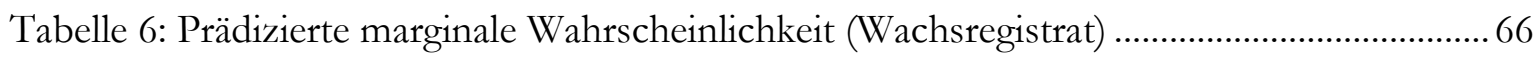

Tabelle 7: Ermittelte Kontaktpunktanzahl je Zahn in statischer Okklusion mittels

Okklusionsfolie.

Tabelle 8: Prädizierte marginale Wahrscheinlichkeit (Okklusionsfolie stat.)

Tabelle 9: Ermittelte Kontaktpunktanzahl je Zahn in dynamischer Okklusion nach links mittels Okklusionsfolie

Tabelle 10: Prädizierte marginale Wahrscheinlichkeit (Okklusionsfolie dyn. li.)

Tabelle 11: Ermittelte Kontaktpunktanzahl je Zahn in dynamischer Okklusion nach rechts mittels Okklusionsfolie.

Tabelle 12: Prädizierte marginale Wahrscheinlichkeit (Okklusionsfolie dyn. re)

Tabelle 13: Ermittelte Kontaktpunktanzahl je Zahn in statischer Okklusion mittels Silikonregistrat

Tabelle 14: Prädizierte marginale Wahrscheinlichkeit (Silikon)

Tabelle 15: Befunde des Zugtests mittels Kontaktfolie 70

Tabelle 16: Prädizierte marginale Wahrscheinlichkeit (Kontaktfolie)........................................... 70

Tabelle 17: Ermittelte prozentuale Kaukraftbeteiligung der Gesamtkraft je Zahn in statischer Okklusion mittels T-Scan III

Tabelle 18: Prädizierte marginale Wahrscheinlichkeit (T-Scan stat.)

Tabelle 19: Ermittelte prozentuale Kaukraftbeteiligung der Gesamtkraft je Zahn in dynamischer Okklusion nach links mittels T-Scan III ............................................ 72

Tabelle 20: Prädizierte marginale Wahrscheinlichkeit (T-Scan dyn. li.). 72

Tabelle 21: Ermittelte prozentuale Kaukraftbeteiligung der Gesamtkraft je Zahn in dynamischer Okklusion nach rechts mittels T-Scan III 73

Tabelle 22: Prädizierte marginale Wahrscheinlichkeit (T-Scan dyn. re.) 73 
Tabelle 23: Prädizierte marginale Wahrscheinlichkeiten der Verfahren für eine

Okklusionsänderung an einem beliebigen Zahn 73

Tabelle 24: Prädizierte marginale Wahrscheinlichkeit für Okklusionsänderungen pro Zahn 74

Tabelle 25: Prädizierte marginale Wahrscheinlichkeit für Okklusionsänderungen pro Kiefer 75

Tabelle 26: Marginale Wahrscheinlichkeiten der Messzeitpunkte .................................................... 76

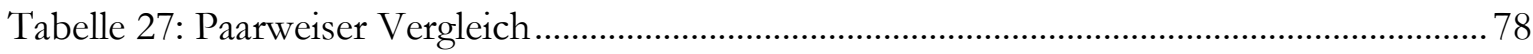

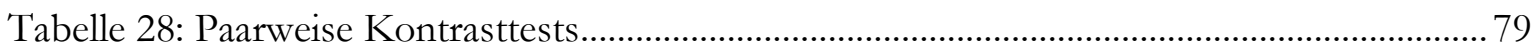

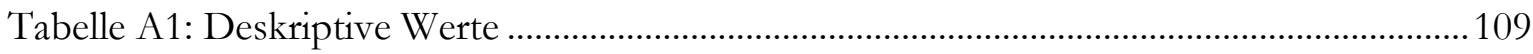

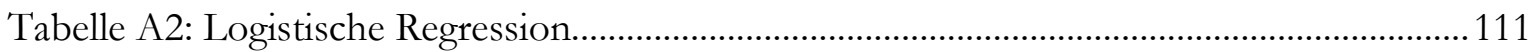

Tabelle A3: Logistische Regression ohne Interaktion mit der Tageszeit .....................................114

Tabelle A4: Logistische Regression zur Okklusionsänderung pro Kiefer ...................................115 


\section{Abkürzungsverzeichnis}

CMD Craniomandibuläre Dysfunktion

DGFDT Deutsche Gesellschaft für Funktionsdiagnostik und -therapie

DGZMK Deutsche Gesellschaft für Zahn-, Mund- und Kieferheilkunde

GEDAS Greifswald Digital Analyzing System

SHIP Study of Health in Pomerania 


\section{Einleitung}

Der Begriff „Okklusion“ beschreibt alle antagonistischen Zahnkontakte zwischen Ober- und Unterkiefer. Die Okklusion und Störungen der Okklusion spielen in der Ätiologie von craniomandibulären Dysfunktionen und in der zahnärztlich-restaurativen Therapie eine entscheidende Rolle. Zur Okklusionsaufzeichnung und -diagnostik kommen in der täglichen zahnärztlichen Routine verschiedene klinisch etablierte Verfahren wie Farbfolien, Kontaktfolien, und Bissregistrate zum Einsatz. Neue digitale Diagnoseverfahren können Okklusionskontakte vermeintlich noch präziser und zeitabhängig aufzeichnen.

Der Beurteilung von statischen und dynamischen Okklusionskontakten am Patienten liegt die Annahme zugrunde, dass diese reproduzierbar sind und reproduzierbar durch die beschriebenen Maßnahmen abgebildet werden können. Geringste Abweichungen der Okklusionsmuster von vermeintlichen Idealen werden häufig als Basis zur Diagnose von Malokklusionen verwendet oder dienen auch der Ableitung von therapeutischen Strategien, wie beim Einschleifen von Zahnersatz oder im Rahmen der Einschleiftherapie bei Patienten mit Funktionsstörungen.

Ziel der vorliegenden Untersuchung war es, mithilfe der digitalen Okklusionsanalyse die Aussagekraft traditioneller Methoden der Okklusionsaufzeichnung (Farbfolien, Kontaktfolien und Bissregistrate) zu untersuchen. Zusätzlich sollte anhand von mehreren aufeinanderfolgenden Okklusionsanalysen (tageszeitabhängig und an unterschiedlichen Tagen) bei gesunden Probanden/-innen überprüft werden, ob die Okklusion per se tatsächlich reproduzierbar und konstant ist. 


\subsection{Okklusion allgemein}

Der Begriff „Okklusion“ beschreibt jeden Kontakt zwischen Zähnen bzw. Zahnersatz des Ober- und Unterkiefers (Ahlers et al. 2006; Clark und Evans 2001; Keith 2017; Türp et al. 2008). Hierbei werden Kontakte der Zähne ohne Bewegung des Unterkiefers (statische Okklusion) von jenen bei Exkursionsbewegung des Unterkiefers (dynamische Okklusion, früher: Artikulation) unterschieden (Ahlers et al. 2006; Strub et al. 2011b). Wird unter Einnahme der statischen Okklusion ein maximaler Vielpunktkontakt zwischen Ober- und Unterkieferzähnen erreicht, ist von maximaler Interkuspidation die Rede (Ahlers et al. 2006; Schmitter et al. 2010). Bei gewohnter Einnahme der Schlussbisslage ist der Begriff der habituellen Interkuspidation bezeichnend, der wiederum in fakultativer Relation zur maximalen Interkuspidation steht (Ahlers et al. 2006; Fukushima 2016). Daraus lässt sich folgern, dass nicht jede Schlussbisslage mit einem maximalen Vielpunktkontakt einhergehen muss (Strub et al. 2011b). Die Okklusion ist als essenzielle Funktion des stomatognathen Systems einzuordnen, das durch seine Konfiguration eine komplexe Funktionseinheit darstellt (Ciancaglini et al. 2002; Kijak et al. 2017; Kirveskari et al. 1989; Sessle 2005).

Zähne, Zahnhalteapparat, Kiefermuskulatur sowie obere Zungenbeinmuskulatur und Kiefergelenke bilden mit übergeordneten, steuernden nervalen Terminalen des zentralen Nervensystems den stomatognathen Apparat (Kijak et al. 2017; Nelson 2015; Sessle 2005). Ein besonderer Zusammenhang ist zwischen der Okklusion und den Kiefergelenken zu sehen. So wird eine bestimmte Unterkieferposition definiert, bei der sich die seitengleich lokalisierten Kondylen in einer physiologischen Kondylus-Diskus-Relation bei ebenso physiologischer Belastung der assoziierten Gewebe gegenüber dem dorsalen Abhang des Tuberculum articulare befinden (sog. Gelenkzentrik) (Ahlers et al. 2006; Keith 2017). Hierbei ist zu berücksichtigen, dass die zentrische Kondylenposition in über $90 \%$ der Fälle nicht mit der Kondylenposition übereinstimmt, bei der meistens anteriore oder retrale Kondyluslagen zu beobachten sind (Alexander 1996; Gauder 2010; Kirveskari et al. 1989; Nelson 2015). Unter Berücksichtigung der vereinfacht dargestellten Zusammenhänge des stomatognathen Systems, ist der Okklusion eine integrale Aufgabe zuzuschreiben, da sie das Zusammenspiel aller Bestandteile des Kausystems zu einer terminalen, definitiven Bewegung bündelt (Kijak et al. 2017; Sessle 2005). Eine physiologisch fungierende Okklusion dissoziiert entstehende Kaukräfte harmonisch auf die gesamte Zahnreihe und erhält unter Verhütung von Zahnwanderungen oder Elongationen eine gesicherte Bissrelation (Alexander 1996; Gauder 2010; Kirveskari et al. 1989; Nelson 2015).

Edward Hartley Angle widmete sich bereits in den 80er-Jahren des 19. Jahrhunderts der sogenannten Malokklusion, also dem unphysiologischen Antagonismus falsch positionierter Zähne, 
indem er die Einordnung in eine physiologische Bogenform mittels modifizierter, festsitzender Apparatur nach Dr. McGill vornahm (1887). Bedeutend später wurden Überlegungen zu allgemeingültigen Okklusionskonzepten vornehmlich durch die häufige Problematik instabil gelagerter dentaler Totalprothesen vorangetrieben (Nelson 2015; Schuyler 1929). Die Lösung der Problematik wurde im Konzept der „balancierten Okklusion“ gesehen, die bilaterale Kontakte in allen funktionellen Exkursionen der Mandibula gewährleistete und ein Abheben der Prothese hierdurch verhinderte (Nelson 2015). Schnell befürwortete man auch die Übertragung dieses für Totalprothesen funktionierenden Konzeptes auf die Okklusion der natürlichen Bezahnung, kehrte jedoch aufgrund mangelnder wissenschaftlicher Evidenz und Akzeptanz ebenso schnell wieder zu den anfänglichen Überlegungen von Angle zurück (1987).

Später nahm vornehmlich Hellman (Gregory et al. 1941) die ursprüngliche Ambition wieder auf, Konzepte für eine ideale bzw. optimale Okklusion der natürlichen Dentition zu entwickeln. Darauf folgten Überlegungen von Beyron (1969, 1954), D’Amico (1961), Friel (1954), Lucia (1962), Ramfjord und Ash (1967), Stallard und Stuart (1963), Andrews (1972) und Okeson (2019). Sechs dieser aufgeführten Autoren ist in den letzten Jahrzehnten eine größere Bedeutung zugekommen. Hierzu sind Stallard und Stuart, Ramfjord und Ash, Beyron, Andrews und Okeson zu zählen. Stallard und Stuart (1963) fassten drei Prinzipien der ,guten Okklusion“ in einem „gnathologischen Okklusionskonzept“ zusammen. Hierbei weisen alle Seiten- und Eckzähne bei Kieferschluss in maximaler Interkuspidation gleichzeitig Okklusionskontakte auf, wobei der Unterkiefer eine maximal retrale Lage einnimmt (rearmost, upmost, midmost position) (Stallard und Stuart 1963). Weiterhin beschreiben diese Autoren, dass bei Kieferschluss in maximaler Interkuspidation weder Führungs- noch Deflexionskontakte auftreten und bei Exkursionsbewegungen aus dieser Position, die Eckzahnführung eine sofortige Disklusion der Seitenzähne bewirkt (Stallard und Stuart 1963).

Ramfjord und Ash (1966) definierten eine „normale Okklusion“ als jede Situation, die unter expliziter Berücksichtigung der physiologischen Adaptionsfähigkeit des gnathologischen Systems in Abwesenheit von Krankheit gefunden werden kann. Eine teilweise Abkehr von starren Okklusionskonzepten wird hierdurch bereits verdeutlicht. Dennoch sehen Ramfjord und Ash (1966), genau wie Stallard und Stuart (1963), die Position der Unterkieferkondylen „in ihrer obersten und rückwärtigsten Position“ als „Grundlage für eine korrekte Ausrichtung des gesamten Kausystems“. Außerdem fordern die Autoren stabile Kieferverhältnisse bei Okklusionskontakten in retraler Kieferlage, Freiheit in Zentrik für den Unterkiefer, geringfügige, gleithindernisfreie exzentrische Bewegungen aus Interkuspidations- wie auch retraler Position in geringem Maße, den Erhalt der vorhandenen Vertikaldimension, die okklusale Führung von der Arbeitsseite in die maximale Interkuspidation, Okklusionskontakte ohne Weichteilbehinderung, 
horizontale Schub- und Druckfreiheit der Zähne. Ein vertikaler Überbiss und die sagittale Frontzahnstufe stellen ebenso wie die Steilheit von Schneidezahn- und Höckerführung keine wichtigen Faktoren dar (Ramfjord und Ash 1967).

Beyrons Konzept einer funktionell optimalen Okklusion erweist sich bis heute vor allem als Grundlage der prothetischen Behandlung als aktuell (Hugger et al. 2006). Nach diesem Konzept solle der Unterkieferschluss in maximale Interkuspidation bei zentrischer Unterkieferposition und okklusaler Störungsfreiheit zu vollführen sein und alle Seitenzähne gleichmäßige Kontakte aufweisen. Die okklusal eintreffenden Kräfte verteilen sich über Approximal- und gleichmäßige Seitenzahnkontakte. Aus der maximalen Interkuspidation ist ein geradliniger Rückschub des Unterkiefers von durchschnittlich etwa $1 \mathrm{~mm}$ möglich. Jeder Zahn wird nur soweit belastet, wie es für eine physiologische Stimulation der parodontalen Gewebe möglich und nötig ist. Die Krafteinwirkung eines Zahnes erfolgt längsaxial. Bei Laterotrusionen weisen nur die Zähne der Arbeitsseite (Gruppenführung) und bei Vorschub nur die Frontzähne Kontakt auf. Exzentrische Bewegungen des Unterkiefers sind störungsfrei möglich, da die Kauflächen flach konfiguriert sind. Es ist von einem ausreichenden Interokklusalraum auszugehen (Beyron 1969, 1954). Andrews (1972) prägte vor allem aus kieferorthopädischer Sicht den Begriff der „,idealen Okklusion“ und stellte mit seinem Werk „Sechs Schlüssel der Okklusion“ eine Orientierung für eine stabile (statische) Okklusion nach kieferorthopädischer Behandlung auf. Hiernach müsste eine korrekte Molarenbeziehung der Ober- und Unterkiefermolaren gegeben sein, eine interindividuelle Angulation der Zahnkronen berücksichtigt werden, Zahnrotationen ausgeschlossen sein, enge Approximalkontakte (kein Lückenstand) vorliegen und die Spee'sche Kurve flach konfiguriert sein (Andrews 1972). Gegenwärtig zeigt sich zunehmend die Auffassung, dass eine störungsfreie Kieferfunktion und die Abwesenheit von Krankheitsbildern eine gute Okklusion definieren.

Okeson (2019) definiert für eine „optimale funktionelle Okklusion“ unter diesem Aspekt folgende Kriterien: Bei geschlossenem Kiefer liegt der Condylus mandibulae in seiner kranialsten und anteriorsten Position gegenüber dem distalen Abgang des Tuberculum articulare. Zwischen den gegenüberliegenden Strukturen befindet sich der Discus articularis. In dieser Kieferlage treten gleichzeitig und gleichermaßen statische Okklusionskontakte aller Zähne auf, wobei die Frontzähne schwächere Kontakte als Seitenzähne zeigen. Bei Zahnkontakt auftretende Kräfte führen zu einer axialen Belastung. Bei Seitwärtsbewegung des Unterkiefers kommt es zu zahngeführten Kontakten der Arbeitsseite und damit zur sofortigen Disklusion der Nichtarbeitsseite. Die Eckzahnführung wird bevorzugt. Bei Unterkiefervorschub führen die Frontzähne, was ebenfalls eine 
sofortige Disklusion der Seitenzähne erzeugt. Eine aufrechte Kopfposition führt zu stärkeren Okklusionskontakten der Seitenzähne und zu schwächeren der Frontzähne (Okeson 2019).

Bis heute herrscht Uneinigkeit hinsichtlich einer korrekten Registrierung und Analyse der verschiedenen Okklusionsformen, was die teilweise unterschiedlichen, gar konträren Definitionen bzw. stetigen Veränderungen der Terminologie zur Okklusion erklärt (Becker und Kaiser 1993; Türp et al. 2008). Die aktuell etablierten Okklusionskonzepte sind rein theoretischer Natur und formulieren dadurch fiktive Richtlinien, die bei Abweichungen von der Norm vielfach eine Therapieindikation fordern (Alt et al. 2017). Die Vielzahl der aufgeführten Überlegungen macht deutlich, dass allgemeingültige Konzepte schwerlich in der Lage sind, die „physiologische“ Okklusion des funktionsgesunden Individuums mit sämtlichen Normvarianten darzustellen (Alt et al. 2017; Hugger et al. 2006).

\subsection{Dynamische Okklusionskonzepte}

Insgesamt können vier dynamische Okklusionskonzepte bzw. Okklusionsmuster unterschieden werden. Hierbei handelt es sich um die frontzahngeschützte, die eckzahngeschützte, die unilateral balancierte und bilateral balancierte Okklusion (Ahlers et al. 2006; Strub et al. 2011b).

\subsubsection{Frontzahngeschützte Okklusion}

Die frontzahngeschützte Okklusion, auch Frontzahn- oder Front-Eckzahn-Führung, beschreibt das Zustandekommen derjenigen dynamischen Okklusionskontakte, die bei der Bewegung des Unterkiefers nach ventral (Protrusion) sowie bei Bewegungen einer Unterkieferseite von der Medianebene weg (Seitschub) an Ober- und Unterkiefer-Frontzähnen entstehen (Ahlers et al. 2006; Beyron 1954; Okeson 2019; Strub et al. 2011b) (Abb. 1).

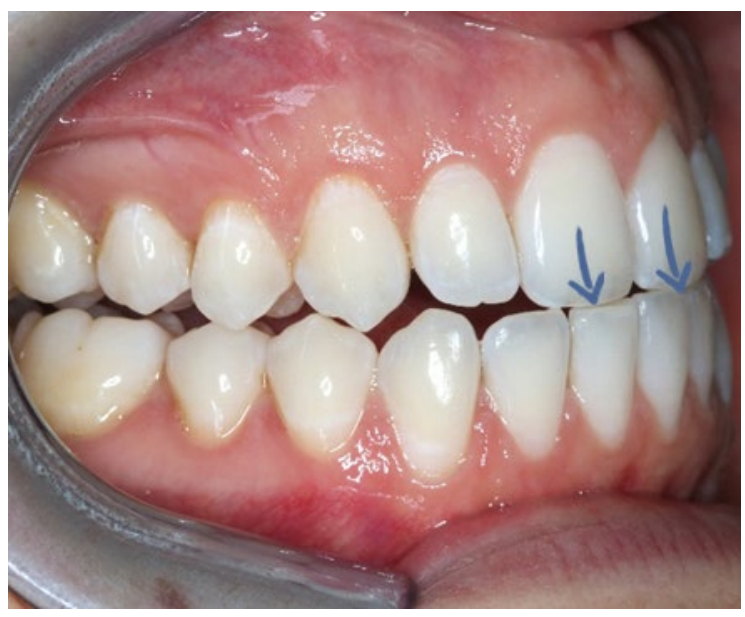

\section{Abbildung 1: Frontzahngeschützte Okklusion.}


Hierbei ist festzuhalten, dass alle Zähne bis auf die Front- bzw. Front- und Eckzähne bei der Latero- und Protrusion sofort diskludieren und daher von nicht-axialen Schubkräften weitestgehend geschützt sind (Koeck et al. 1995; Ramfjord und Ash 1967; Strub et al. 2011a). Eine ausschließlich front-/eckzahngeschützte Okklusion wird bei etwa $10 \%$ (Utz 1982) bis $50 \%$ (Frömder 1989) aller natürlichen Gebisse beobachtet (Koeck et al. 1995). In den meisten Fällen findet sich die kombinierte Front-Eckzahn-Führung, deren Funktion auf die Disklusion der Seitenzähne bei jeglicher exkursiver Bewegung des Unterkiefers abzielt. In der restaurativen Zahnmedizin kommt dieses Okklusionsschema häufig als therapeutisches Okklusionskonzept zum Einsatz (Alt et al. 2017; Wiskott und Belser 1995). Die Zielsetzung dieses Führungskonzeptes ist die Ausschaltung der schädigenden, transversal gerichteten Belastungskomponenten. Zusätzlich wird ein wechselseitiger Schutz der Front- bzw. Eckzähne durch die Seitenzähne erreicht (Okeson 2019), die bei maximaler Interkuspidation für eine geringfügige Disklusion der Frontzähne $(30 \mu \mathrm{m})$ sorgen (Koeck et al. 1995).

\subsubsection{Eckzahngeschützte Okklusion}

Die eckzahngeschützte Okklusion, auch Eckzahn-Führung, beschreibt die ausschließliche dynamische Okklusion der Ober- und Unterkiefer-Eckzähne bei Protrusion und Laterotrusion (Ahlers et al. 2006; Okeson 2019). Hierbei diskludieren alle übrigen Zähne sofort, wodurch ebenfalls der Schutz vor nicht axialen Schubkräften bei allen Zähnen außer den Eckzähnen gewährleistet wird (Okeson 2019; Pasricha et al. 2012) (Abb. 2). Eine rein bilaterale Eckzahnführung wurde in der Untersuchung von Panek et al. (2008), die 834 Probanden/-innen untersuchten, bei $26 \%(\mathrm{n}=217)$ Teilnehmerinnen und Teilnehmern beobachtet. Unilateral eckzahngeschützte Okklusionen waren bei $18 \%$, also bei 150 Probanden/-innen zu beobachten (Ahlers et al. 2006; Bernhardt et al. 2017; Panek et al. 2008). Diese Okklusionsmuster kommen ebenfalls in der restaurativen Zahnmedizin als therapeutisches Konzept - vor allem bei festsitzenden Versorgungen - zum Einsatz.

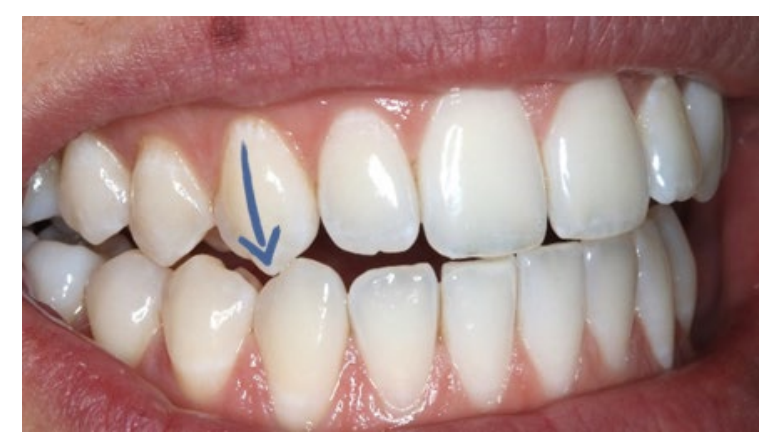

Abbildung 2: Eckzahngeschützte Okklusion. 


\subsubsection{Unilateral balancierte Okklusion}

Die unilateral balancierte Okklusion, auch Gruppenführung genannt, beschreibt die dynamische Okklusion aller sich auf der Arbeitsseite (Laterotrusionsseite) befindlichen Antagonistenpaare (Spee 1890). Eckzähne, Prämolaren und Molaren führen in sogenannten Gruppenkontakten die Unterkieferbewegung zur Seite, während die Frontzähne und sämtliche Zähne der kontralateralen Seite diskludieren (Ogawa et al. 2001, 1998; Singh et al. 2013; Strub et al. 2011b) (Abb. 3 \& 4). In der Studie von Panek et al. (2008) konnte dieses Okklusionsmuster mit einer Prävalenz von $41 \%$ festgestellt werden, was zugleich den größten Anteil darstellte. Ogawa (1998, 2001) und Singh (2013) beschrieben eine vorliegende Gruppenführung in $45 \%$ des untersuchten Probandengutes.

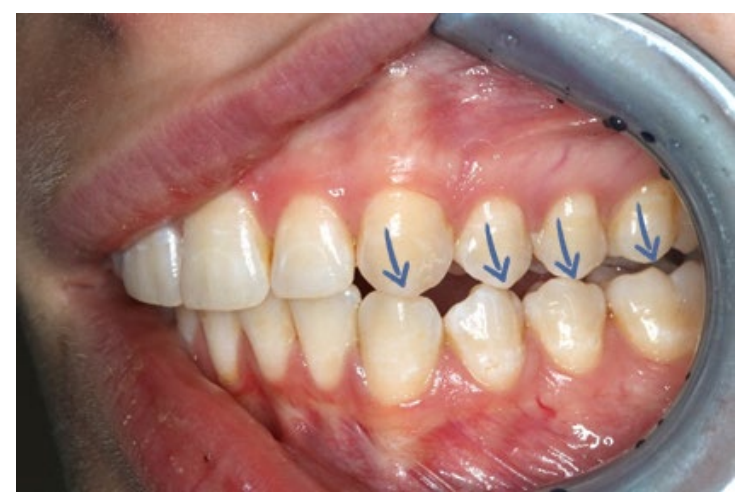

\section{Abbildung 3: Unilateral balancierte Okklusion in vivo.}
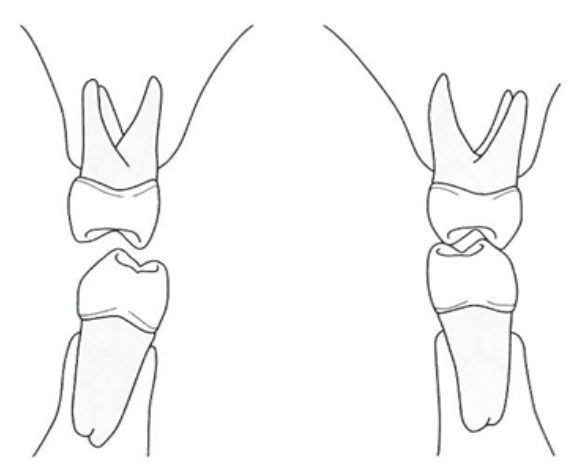

\subsubsection{Bilateral balancierte Okklusion}

Die bilateral balancierte Okklusion wird dadurch charakterisiert, dass alle Unterkieferbewegungen von gleichzeitigen und gleichmäßigen Zahnkontakten begleitet werden. Dies gilt sowohl für protrusive als auch für laterotrusive Bewegungen, in denen sogenannte Protrusionsfacetten der Seitenzähne in der Protrusionsbewegung und Laterotrusions- bzw. Mediotrusionsfacetten in der Laterotrusions- bzw. Mediotrusionsseite (Balanceseite) die Okklusion stabilisieren (Utz 1982) (Abb. 5 \& 6). Das beschriebene Konzept erweist sich im natürlichen Gebiss als nur schwer umsetzbar und kommt vornehmlich für die Totalprothetik infrage, da ein dentaler Totalersatz bei exzentrischen Bewegungen zusätzlich okklusal stabilisiert werden kann (Rateitschak und Wolf 2015; Strub et al. 2011b). In seltenen Fällen kann jedoch eine bilateral balancierte Okklusion einen gelenkprotektiven Charakter haben (Rateitschak und Wolf 2015). 
Panek et al. (2008) konnten lediglich bei $9 \%$ ihrer Versuchspersonen das bilateral balancierte Okklusionskonzept feststellen.
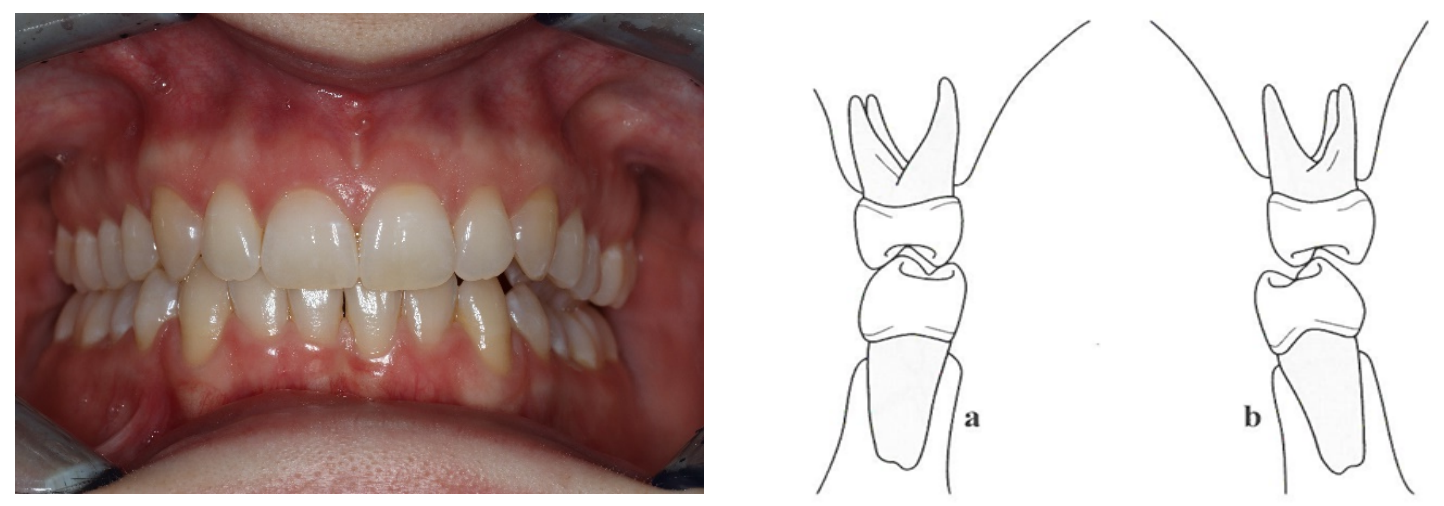

\section{Abbildung 5: Bilateral balancierte Okklu- Abbildung 6: Bilateral balancierte Okklu- sion in vivo. \\ sion. Quelle: Strub et al. (2011). Die Verwen- dung erfolgt mit freundlicher Genehmigung der Quintessenz Verlags-GmbH.}

\subsection{Vergleich der dynamischen Okklusionskonzepte}

Die vorangestellten Definitionen der verschiedenen Okklusionskonzepte auf Basis klinischer Untersuchungen mit unterschiedlichsten Ergebnissen machen vor allem unter Berücksichtigung der vergangenen zwei Jahrzehnte deutlich, welche Rolle die Erforschung der Okklusion für die Funktion des Kauorgans gespielt hat und auch fortlaufend spielen wird. Die Überprüfung bzw. Neubewertung alter Dogmen ist hierbei sicherlich bedeutsam (Bernhardt et al. 2017; Ogawa et al. 1998).

Die Studie der Arbeitsgruppe Panek et al. (2008) verdeutlicht die Prävalenz der verschiedenen Okklusionsmuster und konnte zeigen, dass die bis dato als physiologische Variante bezeichnete und therapeutisch geforderte front-/eckzahngeschützte Okklusion unter laterotrusiver Bewegung des Unterkiefers nicht die höchste Prävalenz im untersuchten Probandengut aufweist. Aufgrund dieser Ergebnisse ist die Forderung nach einer neuen Bewertung der physiologischen Okklusion und entsprechender Pathologien gerechtfertigt (Bernhardt et al. 2017). Dennoch muss im Vergleich der verschiedenen Konzepte immer der kurative Grundgedanke berücksichtigt werden. So ist die grundsätzliche Forderung der front-/eckzahngeschützten Okklusion sowohl mechanisch als auch klinisch betrachtet die schonendste und auch gelenkprotektivste Konzeption eines neutral verzahnten Gebisses, da sie jegliche exzentrische Belastung des kautragenden Anteils bei Unterkieferexkursion entkoppelt (Pasricha et al. 2012; Sidana et al. 2012). 
Wie zuvor beschrieben, kann jedoch auch in Einzelfällen eine sehr seltene Führungsvariante, nämlich die bilateral balancierte Okklusion, hinsichtlich des Kiefergelenkes protektiven Charakter haben (Rateitschak und Wolf 2015). Aus dieser Aussage muss abermals gefolgert werden, dass die grundsätzliche Zielsetzung, jedes Gebiss durch die Erstellung einer front-/eckzahngeschützten Okklusion zu therapieren, obsolet ist (Ogawa et al. 1998; Pasricha et al. 2012; Rinchuse et al. 2007). Vielmehr muss die Therapie individuell auf den Patienten zugeschnitten sein und eine eventuell pathologische Okklusion zu einem Anfangsbefund rekonstruiert werden, um kausal zu behandeln und langfristig stabile Verhältnisse schaffen zu können (Sidana et al. 2012). Die Transferleistung, eine ursprüngliche Okklusion vor allem unter dynamischen Aspekten zu rekonstruieren, ist jedoch vielfach sehr schwierig und in bestimmten Fällen gar unmöglich, was wohl initial die Forderung der allgemeingültigen kurativen Konzepte erklärt (Sidana et al. 2012).

Heute muss initial vor jeder konservierenden, prothetischen, kieferorthopädischen und chirurgischen Therapie die Frage nach der Gestaltungsmöglichkeit der dynamischen Okklusion gestellt werden (Parker 1993). In der Therapieplanung ist bereits häufig festzustellen, dass eine Front-/Eckzahnführung nicht ohne Weiteres realisierbar ist und sie darüber hinaus in vielen Fällen auch zuvor nicht vorlag (Bernhardt et al. 2017; Pasricha et al. 2012; Sidana et al. 2012). Panek et al. (2008) untersuchten 834 Probanden/-innen aus einer randomisierten Gruppe von 1.500 Breslauer Arbeitern im Rahmen einer prophylaktisch-medizinischen Untersuchung. Registriert wurde die zahngeführte exkursive Unterkieferbewegung mit einer herkömmlichen Okklusionsfolie, die farbliche Markierungen auf den beteiligten Zähnen hinterlässt. Das Probandengut wurde hinsichtlich folgender fünf verschiedenen Okklusionsschemata untersucht: bilaterale- und unilaterale Eckzahnführung, Gruppenführung, balancierte Okklusion und andere Okklusionstypen. Mit der höchsten Prävalenz von 41 \% wurde die Gruppenführung festgestellt, eine rein bilaterale Eckzahnführung konnte bei $26 \%$, die unilaterale in $18 \%$, die bilateral balancierte Okklusion bei $9 \%$ und andere Okklusionsmuster bei $6 \%$ der untersuchten Probanden/-innen festgestellt werden. Neben der Tatsache, dass zwischen den Geschlechtern keine signifikanten Unterschiede festzustellen waren, fiel vor allem die Abnahme der bi- und unilateralen Eckzahnführung mit dem zunehmenden Alter der Probanden/-innen auf. Umgekehrt stieg der Anteil der gruppengeführten dynamischen Okklusion anteilig mit dem Alter an. Das heißt also, dass die Eckzahn-Führung eher bei jüngeren Probanden/-innen festzustellen war, wohingegen bei älteren Probanden/-innen zunehmend eine Gruppenführung vorlag ( $\mathrm{Pa}$ nek et al. 2008). 
Anders verhielt es sich in einer japanischen Studie von Ogawa et. al (1998) an 86 jungen Erwachsenen mit dem Durchschnittsalter von 24,2 Jahren. Alter, Neutralverzahnung, Neutralbezahnung bis zum 2. Molaren, Abwesenheit kieferorthopädischer Behandlungen sowie Abwesenheit höckerersetzender Restaurationen waren Bedingungen für den Studieneinschluss. Lediglich bei 9,3\% der untersuchten Probanden/-innen konnte eine eckzahngeschützte Okklusion festgestellt werden. 45,3\% der jungen Erwachsenen wiesen hier eine Gruppenführung und 41,9\% eine balancierte Okklusion auf (Ogawa et al. 1998). Die Untersuchung wurde in dynamischer Okklusion nach rechts und nach links in den Abständen 0,5, 1, 2 und 3 mm Unterkieferexkursion mittels einer sog. Shimstock-Folie der Dimensionierung 10-15 $\mu \mathrm{m}$ erfasst. Grundsätzlicher Unterschied zur Studie von Panek et. al (2008) lag im strengen Altersrahmen, in der untersuchten Ethnie sowie in der vergleichsweise geringen Probanden/-innenzahl, die womöglich die divergenten Ergebnisse erklären könnten.

Wiegelmann et. al (2015) untersuchten in Angliederung an die SHIP-Studie (Study of Health in Pomerania) 558 Probanden/-innen zwischen 20 und 49 Jahren mit mindestens 20 Zähnen bleibender Dentition. Bei einem Viertel des Probandengutes konnte eine front-/eckzahngeschützte Okklusion festgestellt werden, was den Ergebnissen von Panek et al. (2008) näherkommt. Die Unterschiede dieser tendenziell divergierenden Ergebnisse lassen sich eventuell mit der niederländischen Studie um van't Spijker erklären, die verschiedene Positionierungen der Probanden/-innen bei der Laterotrusionsanalyse untersuchte (van't Spijker et al. 2011). Hierzu registrierte ein geschulter Untersucher bei 52 Probanden/-innen mittels einer $8 \mu \mathrm{m}$ dünnen Okklusionsfolie die Führungskontakte bei seitlicher Unterkieferexkursion in aufrecht sitzender, im Winkel von 45 Grad zurückgelehnter und liegender Körperposition. Die zu untersuchenden Okklusionsbefunde wurden in Front-/Eckzahnführung, Gruppenführung und andere Okklusionsmuster unterteilt. Es konnte festgestellt werden, dass sich das gesamte Okklusionsmuster bei Lageänderung zwar nicht gänzlich veränderte, jedoch die Anzahl der Zahnkontaktänderungen von 0 bis 22 variierte. Bei fast allen Untersuchten waren somit signifikante Veränderungen der Antagonistenpaare in einem gleichbleibenden Okklusionsmuster feststellbar. Vergleichend dazu registrierten Ogawa et. al (1998) ihre Probanden/-innen ausschließlich aufrecht sitzend mit Ausrichtung der Frankfurter-Horizontalen parallel zur Grundebene.

Zusammenfassend kann festgestellt werden, dass die unilateral balancierte und front-/eckzahngeschützten Okklusionsmuster in den populationsorientierten Studien die höchste Prävalenz aufweisen. Zusätzlich ist die Annahme berechtigt, dass sich im Verlauf des Alterungsprozesses vom jungen bis zum späten erwachsenen Gebiss eine Änderung des Okklusionsmusters vom front-/eckzahngeführten zum gruppengeführten Typus vollzieht. 
Bei jeglicher Inspektion des Okklusionsmusters muss neben der Körperhaltung auch der Verzahnungstypus in Ruhe berücksichtigt werden (Baldini et al. 2013; van't Spijker et al. 2011). Unterkieferrück- und vorlagen wirken sich durch die für eine Neutralokklusion konzipierten Zahnreihen völlig divergent zum neutralen dynamischen Verzahnungsmuster aus (Parker 1993). Hierbei sind natürlich bereits im jungen Erwachsenenalter Gruppenführungen festzustellen, weil es die Zahnmorphologie und -zuordnung gar nicht anders zulässt (Koeck et al. 1995). Auf die Studie von Ogawa et al. (1998) Bezug nehmend kann vermutet werden, warum besonders in Studien aus Japan und Korea, wo zu hohen prozentualen Anteilen besonders Unterkiefervorlagen anzutreffen sind, zu diesem Thema häufig andere Ergebnisse erscheinen als in europäischen Untersuchungen (Graber und Vanarsdall 1999). Hierbei sei jedoch betont, dass Ogawa et al. (1998) nur Probanden/-innen mit natürlicher Verzahnung eines bereits beschriebenen selektierten Probandenkollektivs untersucht haben.

Unter Berücksichtigung der Studienlage zeigt sich, dass nicht wie zuvor gefordert ein einziges Okklusionskonzept für jeden Patienten gleichermaßen zielführend ist (Pasricha et al. 2012; Sidana et al. 2012). Vielmehr sind das Alter (Panek et al. 2008), die geografische Herkunft (Graber und Vanarsdall 1999), die individuelle Konfiguration des kompletten Kauorgans und unbedingt die akribische Exploration des patientenspezifischen Verzahnungsmusters für die Diagnostik und Therapie wegweisend (Panek et al. 2008; Pasricha et al. 2012; Sidana et al. 2012; Wiegelmann et al. 2015).

\subsubsection{Statische Okklusionskonzepte}

Definitionsgemäß beschreibt die statische Okklusion jene Zahnkontakte, die ohne exkursive Bewegungen des Unterkiefers auftreten (Ahlers et al. 2006; Strub et al. 2011b; Türp et al. 2008). Hierbei wird die maximale Interkuspidation von der habituellen Okklusion unterschieden. Erstere beschreibt den maximal erreichbaren Vielpunktkontakt zwischen Ober- und Unterkieferzähnen, zweitere die gewohnheitsmäßig eingenommene Okklusion, auch habituelle Interkuspidation genannt (Engelhardt et al. 2002; Schmitter et al. 2010; Strub et al. 2011b). Grundlage dieser dezidierten Definition ist die Tatsache, dass die maximale Interkuspidation nicht mit der gewohnheitsmäßig eingenommenen Okklusion zusammenfallen muss (Schmitter et al. 2010; Strub et al. 2011b).

Weiterhin kann die statische Okklusion in Zusammenhang mit dem Kiefergelenk beurteilt werden. Hierbei ist die sogenannte Gelenkzentrik von besonderem Interesse. So wird diejenige Unterkieferposition beschrieben, in der sich die bilateral nicht seitenverschobenen Kondylen zum einen in einer physiologischen Kondylus-Diskus-Relation zeigen und zum anderen eine physi- 
ologische Belastung der beteiligten Gewebe vorliegt (Schmitter et al. 2010). Der Kondylus befindet sich im Bezug zur Fossa mandibularis kranio-ventral, also gegenüber dem Tuberculum articulare. Diese zentrische Kondylenposition wird per definitionem unabhängig von den Zahnkontakten definiert (Rateitschak und Wolf 2015; Schmitter et al. 2010; Strub et al. 2011b). Ursächlich für diese solitäre Betrachtung ist die bereits in 1.1 „Definitionen zur Okklusion“ angeführte Gegebenheit, dass sich in mehr als 90 \% der Fälle (Strub et al. 2011b), bzw. in der Regel die zentrische Kondylenposition von der Kondyluslage bei habitueller Interkuspidation unterscheidet (Böhm et al. 1995a, 1995b; Lentner et al. 1997; Strub et al. 2011b; Utz et al. 1990). Bei dieser sogenannten Interkuspidationsposition des Kondylus befindet sich der gelenktragende Anteil bei den meisten Individuen weiter anterior oder retral (Strub et al. 2011b). Unter Berücksichtigung der Kondylusposition können statische Okklusionskonzepte definiert werden.

Stimmt die zentrische Kondylenposition mit der Interkuspidationsposition überein, liegt eine sogenannte Punkt-Zentrik (point centric) vor (Sander et al. 2011; Weber 2016). In der überwiegenden Anzahl der Fälle ist der gelenktragende Anteil bei Interkuspidation jedoch weiter retral führbar, als es der eigentlichen Gelenkzentrik entspricht. Hierbei handelt es sich um einen unphysiologischen Zustand, der als „RUM-Position“ des Kondylus definiert wird (Palaskar et al. 2013; Weber 2016). Diese Rearmost-uppermost-midmost (RUM)-Position beschreibt die Führbarkeit oder Eigenbewegung des Kondylus zur Fossa articularis. Somit wird hier die hinterste, mittigste und oberste Lagebeziehung des Kondylus zur Fossa beschrieben, die vor allem in der hintersten/obersten Richtung das sogenannte retrodiskale Gewebe komprimiert (Palaskar et al. 2013; Strub et al. 2011b; Weber 2016). Das Konzept der Freiheit in Zentrik, auch freedom in centric, versteht sich als therapeutisches Okklusionskonzept (Sander et al. 2011; Weber 2016). Hierbei lässt sich der Unterkiefer ohne Veränderung der Vertikaldistanz der Ober- und Unterkieferdentition und unter Wahrung der Okklusionskontakte etwa $1 \mathrm{~mm}$ nach anterior schieben, bevor die Seitenzähne diskludieren (Sander et al. 2011; Strub et al. 2011b). Dieses Konzept wird vornehmlich in der Schienentherapie berücksichtigt und findet besonders bei der sogenannten MichiganSchiene Anwendung (Strub et al. 2011b; Weber 2016).

\subsection{Okklusionsbefunde}

Die Befundung der Okklusion stellt jeden Behandler unter Berücksichtigung der zuvor angeführten komplexen und ausdifferenzierten Zusammenhänge vor eine große Herausforderung (Patel und Alani 2015; Sharma et al. 2013). Nicht nur die elementare Kenntnis über die komplexen gnathologischen Zusammenhänge in der Theorie, sondern unbedingt auch die korrekte klinische Sicherung der okklusalen Verhältnisse sowie die Katalogisierung der Ergebnisse zur 
Reevaluation gewährleisten einen patientenorientierten Therapieansatz (Sharma et al. 2013; Takai et al. 1995, 1993). Hinsichtlich der Funktionsdiagnostik ist die Darstellung der Okklusion obligat (Okeson 1995), zugleich stellt sie aber auch einen unverzichtbaren Bestandteil in der Qualitätskontrolle restaurativer oder konservierender Arbeiten aus zahnärztlicher und -technischer Sicht dar (Sharma et al. 2013; Thumati 2015). So vielfältig die Nomenklatur des Oberbegriffs „Okklusion“ erscheint, so zahlreich sind auch die Erfassungsmethoden (Sharma et al. 2013). Hierbei gilt es sicherlich immer die Historie der Okklusionsforschung von den Anfängen bis zur heutigen Zeit zu berücksichtigen, da einige Methoden den gegenwärtigen Ansprüchen hinsichtlich der Genauigkeit nicht mehr genügen (Anderson et al. 1993; Kerstein 2008a). Im nachfolgenden sollen die gängigsten Verfahren aus der klinischen Anwendung beschrieben werden.

\subsubsection{Farbfolien}

Das gängigste Mittel zur klinischen Analyse der okklusalen Kontaktpunktverhältnisse stellen sicherlich Farbfolien dar, die bereits 1953 durch industrielle Fertigung in die tägliche zahnärztliche Behandlung implementiert werden konnten (Okeson 2019). Ziel dieser Folien war von Beginn an die einfache klinische Sichtbarmachung der Kontaktpunktbeziehung mit der Herausforderung, die Okklusion durch dieselbe Maßnahme nicht nennenswert zu verändern (Reiber et al. 1989). Grundvoraussetzung war somit die kleinstmögliche Materialstärke (Schelb et al. 1985) mit bestmöglichen Markierungseigenschaften und die In-vivo-Anwendbarkeit (Saraçoğlu und Ozpinar 2002). Der Ursprung aller farblich markierender Okklusionsfilme liegt im sogenannten Okklusionspapier (Nayar und Babu 2007). Es wurde vor allem mit dem Anspruch entwickelt, drucksensitiv verschiedene Kaukräfte in verschiedenen farblichen Schattierungen darzustellen (Saraçoğlu und Ozpinar 2002; Sharma et al. 2013). Diese progressive Farbtönung sollte störende und zu starke Kontakte sicher detektieren. Aufgrund der fortschreitenden Produktionsentwicklung und immer höheren Anforderungen seitens der Wissenschaft, konnte eine stetige Weiterentwicklung der Materialien hinsichtlich Dimensionstreue und zuverlässiger Markierungseigenschaft gelingen (Okeson 2019). Laut Herstellerangaben soll sich die Urform der Farbfolien, das Artikulationspapier (Abb. 7), besonders zur Darstellung der statischen Okklusion eignen (Okeson 2019). Durch die spongiöse Struktur eines weichen Vliesträgers soll die Möglichkeit gegeben sein, die gespeicherte Farbe druckadaptiert auf die Kontaktflächen abzugeben. So werde bei starken Kontakten, die einen hohen Aufbissdruck implizieren, mehr Farbe abgegeben (Saraçoğlu und Ozpinar 2002; Schelb et al. 1985; Sharma et al. 2013). Leichte Kontakte hingegen lösen durch einen geringeren Kaudruck nur wenige Farbpartikel aus dem Material (Reiber et al. 1989). 
Aus der klinischen Anwendung geht unter Verwendung dieses Materials ein farbschattiertes Kontaktpunktmuster hervor, in dem Kontakte mit hohem Druck als dunkle und Kontakte mit niedrigem Druck als helle Markierungen zu erkennen sind (Saraçoğlu und Ozpinar 2002). Besonderes Augenmerk wurde bezüglich der Materialzusammensetzung auf die Anwendung in der speichelbenetzten Mundhöhle gelegt. Um Kontaktpunkte auf befeuchteten Okklusalflächen sichtbar zu machen, behilft sich der Marktführer Bausch des Haftvermittlers Translucase (Schelb et al. 1985; Sharma et al. 2013).

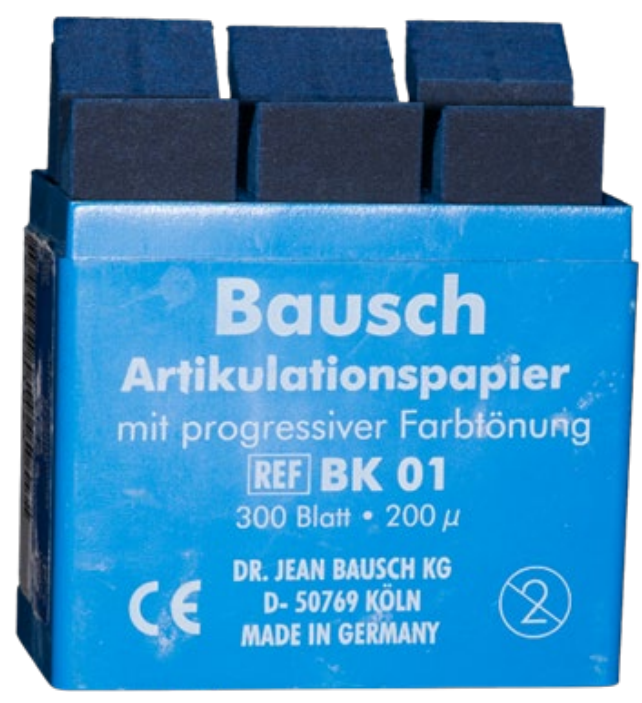

Abbildung 7: Artikulationspapier. Artikulationspapier $200 \mu \mathrm{m}$ (Dr. Jean Bausch GmbH \& Co. KG, Köln, Deutschland)

Hierdurch soll ein zuverlässiges Kontaktpunktmuster auf speichelbenetzten Keramik- und Metalloberflächen gewährleistet sein (Schelb et al. 1985). Die klinisch üblichen Materialstärken belaufen sich auf 100-200 $\mu \mathrm{m}$, was einer annähernden Bisssperrung in dieser Größenordnung gleichkommt und ebenfalls eine Darstellungsverzerrung um diesen Betrag von der tatsächlichen Situation bedeutet (Reiber et al. 1989; Schelb et al. 1985; Sharma et al. 2013). Aus diesem Grund liegt die Forderung eines dünner konfigurierten Materials mit annähernd gleichen Farbmarkierungseigenschaften nahe (Schelb et al. 1985). Die Industrie reagierte mit der Entwicklung eines dünneren $(40 \mu \mathrm{m})$, reißfesten Okklusionspapieres (Schelb et al. 1985). Anders als die höherdimensionierten Derivate arbeitet dieses Papier mit beidseitig beschichteten Liquidfarben, die ein präziseres Zeichnungsverhalten aufgrund ihrer geringen Bisssperrung aufweisen sollten (Schelb et al. 1985). Der Hersteller empfiehlt dieses Material auch für metallische und keramische Werkstoffe sowie Kunststoffe in speichelbenetztem Zustand. Die aus Mikrokapseln bestehende Liquidfarbe soll bei geringen Kaudrücken ein kontrolliertes Zerplatzen der Kapseln ermöglichen, was eine topografische Zuordnung auf den Okklusalflächen erlauben soll (Schelb et al. 1985). 
Ein weiteres Material zur instrumentellen Okklusionsanalyse stellt die Okklusionsseide dar. Aufgrund ihrer natürlichen Herkunft ist auch ihre Dimensionierung limitiert (Reiber et al. 1989; Saraçoğlu und Ozpinar 2002). Dennoch bietet die natürliche Basis der Seide durch ihre fibrilläre Architektur aus tubulären Proteinen auch entscheidende Vorteile (Nayar und Babu 2007; Sharma et al. 2013). So zeichnet sie sich neben einer hohen Reißfestigkeit ebenfalls durch eine gute Flexibilität und eine sehr hohe Farbspeicherkapazität aus und liefert durch ihre Textur keine Pseudo-Kontaktpunkte (Nayar und Babu 2007; Sharma et al. 2013). Trotzdem kann auch Seide, wie andere flexible Indikatoren, bei Speichelzutritt keine zuverlässigen Kontaktmuster darstellen (Reiber et al. 1989; Saraçoğlu und Ozpinar 2002; Sharma et al. 2013). Das $80 \mu \mathrm{m}$ dünne Material findet vor allem in der Herstellung von Totalprothesen Anwendung. Der Hersteller verspricht durch die Flexibilität des Materials eine bessere Eingliederung in das Kaurelief mit geringerer Artefaktabzeichnung auf nicht okkludierenden Flächen, empfiehlt jedoch die Anwendung auf labortechnischer Ebene (Nayar und Babu 2007). Neben der Markierungseigenschaft steht, wie bereits erwähnt, unbedingt die Raumforderung des verwendeten Materials im Mittelpunkt (Schelb et al. 1985). Okklusale Prüfmittel können nur dann annähernd zuverlässige Auskünfte über die individuelle Bisssituation liefern, wenn sie nahezu keine Veränderung der Situation in vertikaler Richtung auslösen (Schelb et al. 1985). Aus der Natur rekrutierte Materialien, wie sie zuvor beschrieben wurden, limitieren sich hinsichtlich der Forderung entweder aufgrund ihrer eigenen Beschaffenheit, oder aber aus Gründen der technischen Herstellung (Sharma et al. 2013). Aus diesem Grund forderten Schelb et al. (1985) die Verwendung okklusaler Prüfmittel mit einer Materialstärke von $<21 \mu \mathrm{m}$. Durch die Verwendung von metallisierten und nicht metallisierten Polyestern oder Polyethylenen kann gegenwärtig eine bedarfsorientierte Folienstärke von minimal $8 \mu \mathrm{m}$, die ,ultradünne“ Artikulationsfolie (Abb. 8), über $12 \mu \mathrm{m}$, die klassischen Shimstock-Folie, bis zu $16 \mu \mathrm{m}$, der flexible Gnatho-Film, angeboten werden (Halperin et al. 1982; Sharma et al. 2013). Aufgrund ihrer geringen Dimension lösen synthetisch hergestellte Farbfolien den geringsten Grad der Bisssperrung aus, was die vornehmliche Verwendung in der alltäglichen Praxis begründet (Anderson et al. 1993; Halperin et al. 1982; Schelb et al. 1985).

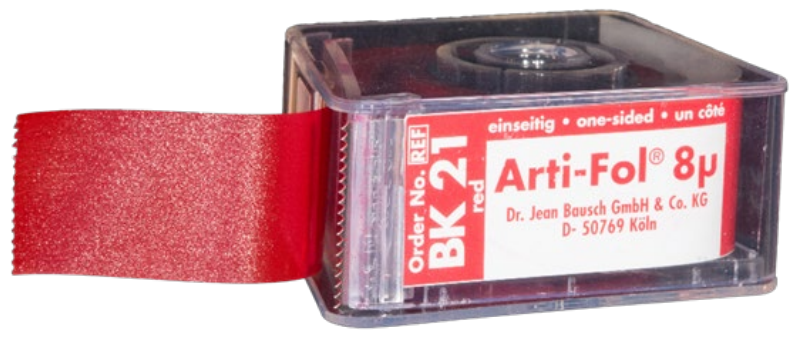

Abbildung 8: Okklusionsfolie. Bausch Arti-Fol $8 \mu \mathrm{m}$ (Dr. Jean Bausch GmbH \& Co. KG, Köln, Deutschland) 


\subsubsection{Kontaktfolien}

Als Alternative zu den zuvor beschriebenen Okklusionsfolien ist die bereits erwähnte Shimstock-Folie gesondert zu betrachten. Bestehend aus einer metallisierten Polyesterfolie können reißfeste Materialstärken von minimal $8 \mu \mathrm{m}$ Stärke angeboten werden (Halperin et al. 1982; Sharma et al. 2013). Als Kontaktfolie besitzt die Shimstock-Folie keine Markierungseigenschaften (Anderson et al. 1993; Solberg 1986). Sie findet besonders in der finalen Justierung von direkten und indirekten prothetischen Versorgungen Anwendung. Der Patient wird hierbei ebenfalls wie bei den markierenden Folien zum Zubiss aufgefordert, jedoch zur Beibehaltung der Schlussbisslage angehalten. Im Anschluss wird die Kontaktfolie unter Zugspannung gesetzt, wodurch das Vorhandensein eines Kontaktpunktes detektiert werden kann (Anderson et al. 1993; Solberg 1986)(Abb. 9). So können durch Verwendung einer $8 \mu \mathrm{m}$ dicken Folie drei Befunde entstehen: die Kontaktfolie lässt sich bei Anbringung der Zugspannung nicht aus dem Kontaktareal entfernen, sie lässt sich unter gewissem Widerstand bei Kontinuitätserhalt der Folie entfernen oder die Entfernung der Folie ist widerstandslos möglich (Engelhardt et al. 2002).

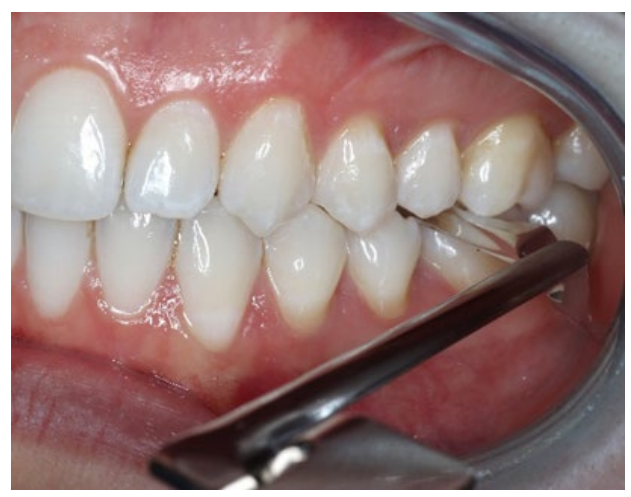

Abbildung 9: Shimstock-Folie. Zugtest der Kontaktfolie (Coltène/Whaledent AG, Schweiz)

Je nach Befund sind folgende Rückschlüsse festzustellen: Lässt sich die Folie bei Anbringung der Zugspannung nicht entfernen, so ist eine antagonistische Kontaktpunktbeziehung von $\leq 8 \mu \mathrm{m}$ anzunehmen. Neben dem Positivbefund des Behandlers ist auch der Patient in der Lage, einen Kontakt wahrzunehmen. Lässt sich die Folie unter Widerstand aus dem Kontaktraum der antagonistischen Zähne entfernen, so ist eine Kontaktpunktbeziehung von > aber annähernd $8 \mu \mathrm{m}$ anzunehmen. Das Ausgleiten der Folie wird zwar vom Patienten registriert, jedoch wird kein Fremdkörpergefühl in vertikaler Dimension wahrgenommen. Lässt sich die Folie widerstandslos aus dem Kontaktraum entfernen, so ist eine wesentlich größere Kontaktpunktbeziehung als $8 \mu \mathrm{m}$ anzunehmen. In diesem Fall ist es dem Patienten eventuell nicht möglich, das ledigliche Ausgleiten der Folie zu registrieren (McDevitt und Warreth 1997). 
Wie bereits erwähnt, fanden und finden die vorgestellten Folien in ihren verschiedenen Konfigurationen in der klinischen Praxis Anwendung, jedoch nicht, ohne zuvor im Rahmen klinischer Studien auf ihre Tauglichkeit und Nutzen untersucht worden zu sein (Anderson et al. 1993; DeLong et al. 2007; Kerstein 2008a; Malta Barbosa et al. 2018; Qadeer et al. 2012; Reiber et al. 1989; Schelb et al. 1985; Sharma et al. 2013). Besonderes Augenmerk lag hierbei damals wie auch heute auf der individuellen Kaukraft. So wurde durch Riise (1982) erstmals in einer klinischen Studie versucht, eine Differenz der okklusalen Kontaktpunktmenge bei starker und schwacher Interkuspidation in verschiedenen Altersklassen festzustellen. Hierzu wurde das Probandengut vier Gruppen zugeordnet, wobei sich die erste Gruppe (junge Erwachsene) aus 30 Probanden/-innen zusammensetzte, deren Altersspanne zwischen 19 und 29 Jahren lag. Die zweite Gruppe (Erwachsene) stellte sich aus 61 Probanden/-innen der Altersspanne 30 bis 69 Jahre zusammen. Die dritte Gruppe (Erwachsene C) wurde von 27 Teilnehmern im Alter von 30 bis 56 Jahren gebildet. Die letzte Gruppe, Erwachsene A, bestand aus 106 Probanden/-innen der Altersspanne 23 bis 69 Jahre. Für die Kontaktpunktanalyse wurde eine $8 \mu \mathrm{m}$ starke markierende Folie verwendet. Mit hoher Signifikanz konnte die Korrelation der Kontaktpunktquantität bei starker Interkuspidation festgestellt werden. Die Kontaktpunkte nahmen also bei Zunahme der aufgebrachten Kaukraft ebenfalls zu (Riise 1982). Weiterhin interessant war die Beobachtung, dass in der Gruppe der jungen Erwachsenen eine signifikant kleinere Kontaktpunktanzahl bei schwacher Interkuspidation gegenüber der Gruppe „Erwachsene“ vorlag (Riise 1982). Überraschenderweise konnte jedoch bei starker Interkuspidation kein signifikanter Unterschied zwischen den Gruppen festgestellt werden (Riise 1982).

In einer weiteren Studie von Riise und Ericsson (1983) wurde untersucht, ob sich ein signifikanter Unterschied bezüglich der Kontaktpunktlokalisation bei habitueller und starker Interkuspidation feststellen lässt. Hierzu wurde das Probandengut in drei Gruppen aufgeteilt, wobei sich die erste Gruppe der, ,jungen Erwachsenen“ aus 30 Teilnehmen zwischen 19 und 29 Jahren, wovon 20 weiblich und zehn männlich waren, zusammensetzte. Die zweite Gruppe der „Erwachsenen“ bestand aus 63 Probanden/-innen im Alter von 30 bis 58 Jahren, wovon 23 weiblichen und 40 männlichen Geschlechts waren. In der letzten Gruppe „Erwachsene A“ befanden sich 42 weibliche und 64 männliche Probanden (insgesamt 106) im Alter von 23 bis 69 Jahren. Alle Teilnehmer wiesen ein vollständig bezahntes und kariesfreies Gebiss auf. Wie auch in der vorhergegangenen Studie, stellten Riise und Ericsson (1983) die Kontaktflächen mit $8 \mu \mathrm{m}$ dicker Markierungsfolie dar. Für die Auswertung wurden die Zahnreihen in Frontzähne, Prämolaren und Molaren gruppiert. 
In der Gesamtheit der Gruppen konnte eine Zunahme der Kontaktflächen bei starker Interkuspidation in Relation zur schwachen Interkuspidation festgestellt werden. Hinsichtlich der Verteilung der Kontaktflächen bei starker und schwacher Interkuspidation konnte kein Unterschied zur Gesamtheit der Kontaktflächen festgestellt werden (Riise und Ericsson 1983). Bezüglich der Symmetrie der Kontaktflächenverteilung konnte ebenfalls keine Differenz gesehen werden. Weiter konnte kein signifikanter Unterschied in den Fällen festgestellt werden, in denen eine höhere Kontaktpunktzahl auf einer Seite vorlag, wenn eine starke oder schwache Interkuspidation durchgeführt wurde (Riise und Ericsson 1983). Riise und Ericsson (1983) folgerten aufgrund ihrer Studienerkenntnisse, dass vor allem die Stabilität der Okklusion bei restaurativen Eingriffen erreicht werden müsse und das Streben nach dem maximalen Vielpunktkontakt nicht mehr als Hauptaufgabe anzusehen sei. Den Autoren zufolge liegt die wahre Restitutio ad integrum in der Rehabilitation der okklusalen Struktur, die sowohl bei starker als auch schwacher Interkuspidation dieselbe Kontaktpunktmenge liefert (Riise und Ericsson 1983).

Basierend auf diesen Erkenntnissen untersuchten McDevitt und Warreth (1997) ihre Patienten hinsichtlich einer bilateralen Kontaktpunktsymmetrie, wie sie in den gängigen Okklusionsmodellen gefordert wurde. Hierzu untersuchten sie 38 Probanden/-innen mit mindestens 24 Zähnen in physiologischer Okklusion. Keine/-er der untersuchten Probanden/-innen wies jedoch eine symmetrische Kontaktpunktverteilung auf. Augenscheinlich war zusätzlich die geringe Anzahl der Kontaktflächen im posterioren Bereich (McDevitt und Warreth 1997).

Bereits Anfang der 70er-Jahre führten Anderson und Myers eine der heutigen instrumentellen Okklusionsanalyse nahekommenden Untersuchung durch. Darin wurden 32 erwachsene Probanden/-innen in der Altersspanne von 20 bis 59 Jahre einbezogen, deren Gebiss zwar vollständig zu sein hatte, jedoch mit konservierenden und/oder prothetischen Versorgungen versehen sein durfte. Hinsichtlich der Auswertung muss angemerkt werden, dass die Befundung der Okklusion bei Vorhandensein von dritten Molaren nicht berücksichtigt wurde. Myers und Anderson führten bei allen Probanden/-innen Alginatabformungen des Ober- und Unterkiefers durch und registrierten die habituelle Interkuspidation mittels Wachsaufbissplatte. Im Anschluss wurden die gewonnenen Alginatabformungen mit Gips ausgegossen und Kiefermodellpaare hergestellt. Diese Modelle wurden unter Berücksichtigung der registrierten habituellen Okklusion mittels Wachsbiss in einen Kiefergelenkssimulator (Artikulator) eingebracht und instrumentell mittels Farbfolie ausgewertet. Es konnte nach Untersuchung aller Modellsituationen keine optimale Interkuspidation festgestellt werden. Auffallend war lediglich die häufig nicht antagonistisch abgestützte Frontzahnsituation. Hinsichtlich der Kontaktflächensymmetrie wurden keine Erkenntnisse formuliert (Myers und Anderson 1971). 


\subsection{Quetschbisse}

\subsubsection{Wachs}

Als klassisches Registriermaterial kann hartes oder weiches Modellierwachs z. B. Beauty Pink Wax (Integra LifeSciences, Princeton, NJ, USA), oder HS-Modellierwachs (Schuler Dental, Ulm, Deutschland) bzw. HS-Modellierwachs (Henry Schein Inc., Melville, USA) verwendet werden. HS-Modellierwachs ist unter anderem in einer 175 x $80 \mathrm{~mm}$ messenden und 1,25 $\mathrm{mm}$ starken Wachsplatte erhältlich. Das Material setzt sich aus einer Mischung von Kohlenwasserstoff- und Paraffinwachsen zusammen. HS-Modellierwachs zeichnet sich durch leichte Elastizität und geringes Rückstellvermögen aus. Der Erstarrungspunkt liegt bei $55^{\circ} \mathrm{C}$. Da der Schmelzpunkt des Wachses zwischen 54 und $61^{\circ} \mathrm{C}$ liegt, wird ein Wasserbad mit niedrigerer Temperaturkalibrierung $\left(42^{\circ} \mathrm{C}\right)$ zur Anwärmung des Materials empfohlen. Anschließend lässt sich die leicht angewärmte Wachsplatte vorab an der Oberkieferzahnreihe adaptieren bis der/die Patient/-in unmittelbar danach zum habituellen Zahnreihenschluss aufgefordert wird. Nach kurzer Verweildauer kann das Wachsregistrat entnommen und die erfassten Zahnabdrücke zur dreidimensionalen Darstellung der Okklusalfläche herangezogen werden. Hinsichtlich der Okklusionsbeurteilung ist vor allem die Impressionstiefe der Zahnabdrücke interessant, die vor einer Lichtquelle beurteilt wird (Ehrlich und Taicher 1981). Myers und Anderson (1971) unterteilten die Kontaktqualitäten in Suprakontakte, Kontakte und annähernde Kontakte. Impressionen mit geringer oder erahnbarer Tiefe wurden als annähernde Kontakte angesehen. Solche Impressionen, die bei Durchleuchtung der Lichtquelle einen kontinuierlich-transluzenten Bereich aufwiesen, wurden als Kontakte gewertet. Suprakontakte wurden anhand einer perforierten Wachsschicht identifiziert. Myers und Anderson (1971) konnten im Rahmen dieser Registrierung die zuvor genannte und häufig beobachtete fehlende Frontzahnkontaktbeziehung aufzeigen. Ehrlich und Traicher (1981) beobachteten im Zuge der Wachsregistrierung die Zunahme von Kontakten in posteriorer Richtung von Prämolaren zu Molaren, indem sie 29 Probanden/-innen mit einer vollbezahnten Angle-Klasse-I-Verzahnung auf Wachsregistrate beißen ließen. Ciancaglini et al. (2003) ermittelten unter Verwendung von Wachsregistraten das Vorliegen von asymmetrischen Kontaktpunktverteilungen und formulierten basierend auf ihren Ergebnissen die These, dass eine asymmetrische Kontaktpunktverteilung möglicherweise in Assoziation mit der Ätiologie von Kiefergelenksbeschwerden stehe. 


\subsubsection{Silikon}

Auch Silikone eignen sich zur klinischen Okklusionsuntersuchung (DeLong et al. 2007). Hierbei kommen schnellabbindende Registriersilikone z. B. Regisil PB (Dentsply Caulk, Milford, DE, USA), Futar D (Kettenbach, Eschenburg, Deutschland), Registrado X-tra (Voco, Cuxhaven, Deutschland), Exabite II NDS (GC, Tokyo, Japan) zum Einsatz (DeLong et al. 2007; Rateitschak und Wolf 2015). Bei Registrado X-tra (Voco GmbH, 27472 Cuxhaven, Deutschland) handelt es sich um ein additionsvernetzendes Silikon-Abformmaterial zur Bissregistrierung. Es zeichnet sich durch eine hohe Endhärte und eine geringe Abbindezeit aus. Das Silikon verfügt über eine spezielle temperaturgesteuerte Aushärteformel und bindet 40 Sekunden nach intraoraler Applikation ab. Im Anschluss lässt es sich bruchsicher aus der Mundhöhle entnehmen und kann bedarfsgerecht zugeschnitten oder beschliffen werden. Zur okklusalen Bissregistrierung wird das vermengte Silikon auf den oberen oder unteren Zahnkranz des Patienten intraoral appliziert. Das Material lässt sich vor der Abbindereaktion 30 s bei $23^{\circ} \mathrm{C}$ Raumtemperatur verarbeiten und bindet im Anschluss $40 \mathrm{~s}$ ab. Bis zur vollendeten 30. Sekunde muss der Patient folglich die gewünschte Interkuspidationsposition eingenommen haben und dort bis zur vollständigen Abbindung des Materials verweilen. Anschließend kann das ausgehärtete Registrat aus dem Patientenmund entnommen und in eine wässrige Desinfektionslösung getaucht werden. Unter Einhaltung der lösungsspezifischen Einwirkzeit kann das Registrat anschließend entnommen, luftgetrocknet und begutachtet werden. Es ist bei der Verwendung darauf zu achten, dass Materialien wie Latex, Mundspül- oder sonstige Reaktionslösungen die Abbindung des Silikons inhibieren können. Materialspezifisch handelt es sich bei Voco Registrado X-tra um Polydimethylsiloxane mit Platinkatalysatoren. Überempfindlichkeiten gegenüber dieser Inhaltsstoffe sind vor Gebrauch zu erfragen und auf die Anwendung zu verzichten, sofern eine allergische Reaktion zu erwarten ist.

Die aufgeführten, prominentesten Vertreter sind in Gesamtheit den additionsvernetzenden Silikonen zuzuordnen und variieren hinsichtlich ihrer Endhärte, Dimensionstreue, intraoraler Haftbarkeit, Beschleifbarkeit sowie Elastizität und Abbindezeit. Als Mittel der Wahl verschiedenster Studien, erscheinen die Silikone trotz unterschiedlicher Zusätze oder Kompositionen in ihrer klinischen Anwendung immer gleich: Der/Die Proband/-in wird nach Applikation der niedrig viskösen Silikonspur auf die Zahnreihe oder einen individualisierten Materialträger zum Schlussbiss aufgefordert, in dem bis zur Aushärtung des Materials verweilt wird (DeLong et al. 2007; Ziebert und Donegan 1979). Nach abgeschlossener Abbindezeit kann das Registrat entnommen und direkt beurteilt werden. 
Entgegen der Beurteilung des Wachsbisses, wird das Silikonregistrat in einigen Studien mittels Gipskiefermodell und Indikatorfarbe quantitativ visuell befundet. Hierzu erfolgte nach Aufbringen des Silikonschlüssels auf die Zahnreihe des Gipsmodells die Anfärbung der perforierten Areale mittels Farbe (Millstein 1983; Ziebert und Donegan 1979). Auch für Silikonregistrate stellt die transilluminative Befundung heute noch eine etablierte Methode dar (DeLong et al. 2007).

Die Arbeitsgruppe um Woda (1987) untersuchte die okklusalen Kontaktpunktverhältnisse bei 22 erwachsenen, kariesfreien und vollständig bezahnten Probanden/-innen. Mittels SilikonbissRegistraten sollte die individuelle antagonistische Zahnkontaktbeziehung mit möglichst geringer Bisssperrung ermittelt werden. Wie zur Wachregistrierung beschrieben, erfolgte die visuelle Auswertung wiederum vor einer Lichtquelle. Hierbei wurden Perforationen im Bissregistrat als sog. Suprakontakte und Areale dünnster Konfiguration, jedoch ohne Kontinuitätsunterbrechung, als Kontakte bewertet. Hinsichtlich der Ergebnisse konnte eine asymmetrische Kontaktpunktverteilung bei deutlich geringerer Gesamtanzahl festgestellt werden. Vor allem Bezug nehmend auf die bis dato gängigen Okklusionstheorien, deren Forderung eine maximal mögliche Vielpunkt-Kontaktbeziehung implizierte, ließen diese Ergebnisse die Forderung nach weiterer Untersuchung der Okklusion zu (Woda et al. 1987).

Anschließend zeigte Korioth (1990) mittels eines fast identischen Versuchsaufbaus den von Woda (1987) zuvor beschriebenen Sachverhalt einer deutlich reduzierteren Kontaktpunktbeziehung in vivo. Sein Probandengut belief sich mit 54 Teilnehmenden auf mehr als das Doppelte der vorhergegangenen Studie. Anders als Woda (1987) bediente sich Korioth (1990) eines differenten Registriermaterials. Wie bereits im Vorfeld beschrieben, stellt eine algenbasierte Abdruckmasse, das sogenannte Alginat, bis heute das Abdruckmaterial der Wahl bei Anfertigung von Situationsmodellen dar. Korioth (1990) verwendete das Abdruckmaterial im Sinne einer Registriermasse und bewertete perforierte Areale als okklusale Kontakte. Sowohl Woda (1987) als auch Korioth (1990) zeigten eine deutliche Abweichung von Kontaktpunktlage und -anzahl der In-vivo-Situation zu der von Beyron (1969) geforderten „guten Okklusion“. Bei Korioth (1990) wichen die untersuchten Kontaktpunktbeziehungen in den meisten Fällen sogar erheblich von dem durch Beyron postulierten Modell ab. In den meisten Fällen wichen die untersuchten Kontaktpunktbeziehungen sogar erheblich von dem durch Beyron postulierten Modell ab (Korioth 1990). 
Millstein (1984) nahm sich der Tatsache an, dass es unter Berücksichtigung aller bis dato gelieferten Ergebnisse, immer noch an einem sicheren System mangelte, das eine dauerhafte und lagerfähige Okklusionserfassung liefern konnte (Davies et al. 2005; DeLong et al. 2007; Millstein 1984). Aus diesem Grund arbeitete Millstein an einem dreidimensionalen Verfahren, das eine suffiziente und langzeitstabile Okklusionsanalyse ermöglichen sollte. Hierbei stand die Etablierung des Verfahrens selbst im Mittelpunkt, wobei die okklusalen Kontaktpunktbeziehungen in reduzierter Form analysiert wurden. Lediglich die Prämolaren und Molaren der linken Seite des/der Probanden/-in wurden in diesem Versuch hinsichtlich ihrer Kontaktpunktbeziehung untersucht. Millstein (1984) bediente sich eines Registriermaterials aus Silikon, da die Eigenschaften einer geringen Bissbeeinflussung durch ebenso geringen Widerstand beim Zubiss sowie die kurze Abbindezeit des Silikons für seinen Versuchsaufbau ideal erschienen. Die Silikonbisse wurden für die Modelljustierung des jeweiligen Probanden/-innen in einem Artikulator verwendet. Die geschaffene Modellsimulation lieferte anschließend die Basis zur Herstellung von drei Acryl-Harz-Registraten. Die gewonnenen Registrate wurden wiederum unter Zuhilfenahme einer Lichtbox von der Unterseite durchleuchtet und fotografiert. Anschließend erfolgte die Digitalisierung der Filmträger mittels Mikrodensitometrie sowie die computergestützte Befundung. Auf diese Weise konnte der Forscher ein zuverlässiges und reproduzierbares Verfahren etablieren, das neben der Darstellung der Okklusion auch die Archivierungsmöglichkeit lieferte (Millstein 1984).

Aufgrund der vielen Studienergebnisse zur Okklusionsanalyse, drängte sich die Forderung auf, die Okklusion weniger topisch zu betrachten. Vor allem wurde die Implementierung der anderen Kompartimente - wie artikuläre und muskuläre Einheiten des stomatognathen Systems zur Erstellung eines neuen Okklusionsmodells essenziell (Gurdsapsri et al. 2000). Aus diesem Grund versuchte die Arbeitsgruppe um Gurdsapsri (2000) die Auswirkung differenter Kaukräfte auf die antagonistischen Kontaktpunktbeziehungen sowie der Kontaktflächenveränderung der verschiedenen Areale des Zahnbogens zu untersuchen. Hierzu konnte ein Probandenkreis aus zwölf weiblichen und 13 männlichen Teilnehmern im Alter von 24 bis 32 Jahren gewonnen werden. Die Gesamtheit aller Teilnehmenden zeigte eine kariesfreie, suffiziente Dentition. Vorliegende craniomandibuläre Dysfunktionen (CMD) sowie Parodontopathien führten zum Studienausschluss. Der Herausforderung, die individuelle Kaukraft quantitativ reliabel zu untersuchen, wurde mittels elektromyograpischer Kontrolle Rechnung getragen. Die Arbeitsgruppe stellte für den/die jeweiligen Probanden/-in vier Bissregistrate bei differenten Kaukräften her. Die Okklusion wurde bei $10 \%, 30 \%$, $70 \%$ sowie $100 \%$ der maximalen Kaukraft registriert. 
Auch in dieser Untersuchung wurde unter Zuhilfenahme einer Lichtquelle befundet. Bereiche, die $\leq 50 \mu \mathrm{m}$ Sichtstärke im Registrat aufwiesen, wurden als Kontakte bewertet. Zur dezidierten Analyse wurde der Zahnbogen in Frontzähne, Prämolaren und Molaren gruppiert. Die Arbeitsgruppe konnte in ihrer Untersuchung signifikant belegen, dass die Kontaktareale der Prämolaren und Molaren in positiver Korrelation zur Kaukraft stehen. Gurdsapsri et al. (2000) bewiesen damit, dass eine Erhöhung der Kaukraft eine Vergrößerung des Kontaktareals impliziert. Hinsichtlich des Frontzahnsegmentes konnte dieser Zusammenhang nicht festgestellt werden. Weiterhin konnte bezüglich der symmetrischen Kontaktflächenverteilung des Prämolaren- und Molarenbereichs der linken und rechten Seite keine signifikante Differenz befundet werden (Gurdsapsri et al. 2000).

\subsubsection{Polyether}

Polyether finden universell für sämtliche Bereiche der Präzisionsabformung Anwendung. Sie zeichnen sich vor allem durch ihre relativ geringe Schrumpfung und gute Dimensionsstabilität aus (Baba et al. 2000). Durch ihre initial hydrophile Eigenschaft wird vor allem das exakte Anfließen des Materials an feuchte Zahnoberflächen unterstützt (Strub et al. 2011a). Das polymerisationsvernetzende Material weist gegenüber Silikonen eine längere Abbindedauer auf, was eine unbedingte Lagestabilität der Abformmasse obligat macht (Durbin und Sadowsky 1986). Aufgrund der hohen Steifigkeit des Materials, die letztlich das ausgezeichnete Dimensionsverhalten sichert, kommt es bei unvorsichtiger Handhabung häufig zu Rupturen bzw. Kontinuitätsunterbrechungen feinster abgeformter Strukturen. Da neben der exakten, nahezu widerstandslosen Eigenschaft des Registriermaterials vor allem die Befundbarkeit eben dieser feinen okklusalen Kontaktareale gefordert wird, finden Polyether für die Bissregistrierung seltener Anwendung. Der prominenteste Vertreter der polyetherbasierten Bissregistrierungsmaterialien ist Ramitec (3M ESPE, Seefeld, Deutschland) (Durbin und Sadowsky 1986).

Ghazal et al. (2008) konnten im Rahmen ihrer Untersuchung eine nahezu gleiche Dimensionstreue zwischen dem untersuchten Polyether und Silikon feststellen. Nach einer Stunde Lagerung wiesen die getesteten Silikone Futar D (Kettenbach, Eschenburg, Deutschland) und Ramitec (3M ESPE, Seefeld, Deutschland) $23 \pm 4 \mu \mathrm{m}$ bzw. $25 \pm 5 \mu \mathrm{m}$ vertikale Diskrepanz auf. Nach 48 Stunden zeigte Futar D $33 \pm 5 \mu \mathrm{m}$ und Ramitec $30 \pm 5 \mu \mathrm{m}$ vertikale Diskrepanz (Ghazal et al. 2008). Ghazal et al. (2008) kamen daher zu der Schlussfolgerung, dass Polyether - genau wie Silikone - die beste Dimensionstreue gegenüber sämtlichen Materialien aufweisen.

Ähnlich dem zuvor beschriebenen Versuchsaufbau von Gurdsapsri et al. (2000) untersuchten Kumagai et al. (1999) die okklusale Kontaktbeziehung in Relation zu verschiedenen Kaukräften. 
Auch diese Arbeitsgruppe bediente sich einer elektromyographischen Untersuchung zur Ermittlung der individuellen Kaukräfte. 13 männliche und drei weibliche Probanden/-innen stellten sich mit kariesfreiem, vollbezahntem Gebiss unter Ausschluss einer CMD für die Untersuchung zur Verfügung. Auf Basis der vorab registrierten, individuell maximalen Kaukraft wurden fünf Polyether-Bissregistrate bei verschiedenen Kaukräften gewonnen. Bei 20-, 40-, 60-, 80- und $100 \%$ der maximalen Kaukraft wurde für jede/-n Probandin/-en ein Bissregistrat hergestellt. In der Auswertung wurden die Kontaktpunktstärke, die Kontaktarealverteilung bei differenter Kraftaufwendung sowie die Kontaktlokation allgemein berücksichtigt. Des Weiteren wurde eine topische Zuordnung in fünf Areale des Zahnbogens vorgenommen, wobei zwischen anterioren Zähnen, rechter und linker Prämolaren- sowie rechter und linker Molarenregion differenziert wurde. Kumagai (1999) konnte Kontaktpunktveränderungen feststellen, die sich am deutlichsten im Molarenareal manifestierten. Weiterhin konnte eine Änderung geringeren Grades in der Prämolarenregion festgestellt werden. Die kleinste Änderungsrate wurde im anterioren Zahnsegment beobachtet. Die Untersuchung verdeutlichte einen linearen Zusammenhang hinsichtlich der Zunahme der okklusalen Kontaktareale bei zunehmender Kaukraftentwicklung (Kumagai et al. 1999).

\subsection{Digitale Messmethoden}

\subsubsection{T-Scan-System}

Der T-Scan III wird von der Firma Tekscan (Tekscan Inc., South Boston, USA) produziert und erschien 2006 als in dritter Generation weiterentwickeltes System des erstmalig 1987 eingeführten T-Scans (Maness et al. 1987). Ein mit dem Computer verbundenes Handstück nimmt über eine größenvariable Bissgabel Sensorfolien auf, die Kontaktpunkte eines/-r auf den Folienträger beißenden/-er Probanden/-in mittels kleiner Sensoren registrieren. Die zusätzliche Software macht die registrierten Kontaktpunkte auf einem virtualisierten Zahnbogen sichtbar.

Die erste Generation des T-Scans erlaubte initial nur kompromittierte reproduzierbare Ergebnisse. Ursächlich war hierbei sicherlich die Solidität der Sensorfolie, die zur vielfach beschriebenen, deutlichen Verschiebung des Bisses im Sinne einer Latero-Exkursion der Mandibel führte (Boening und Walter 1992; Garrido Garcia et al. 1997; Harvey et al. 1991; Lyons et al. 1992b; Patyk et al. 1989a; Patyk et al. 1989b; Wang et al. 2011; Yamamura et al. 1990). Die folgende Generation des Systems, T-Scan II, sollte explizit diese Unterkieferexkursion unterbinden. Durchgeführte Studien konnten zeigen, dass bei Laterotrusionen sechs differente Kontaktschemata zu finden waren, die in Gänze nicht mit den Modellen der Front-Eckzahn- bzw. Gruppenführung vereinbar waren (Wang et al. 2011). 
Das T-Scan-III-System zeichnet sich durch technische Verbesserungen wie USBSensorhandstück sowie Sensorfolien mit vertikal und horizontal angeordneten Leitern, die einfallende Kräfte in einem Matrixschema erfassen können, aus. Das System setzt sich aus mehreren Komponenten zusammen. Das manuelle Instrument erscheint als Handstück im Sinne einer elektronischen Bissgabel, das via USB-Kabel mit einem stationären oder mobilen Rechner verbunden ist. Variabel sind die mitgelieferten Sensorfolienhalter. Je nach Patientensituation kann aus den zwei verschiedenen Konfigurationen, nämlich small und large, ausgewählt werden. Komplementär zur jeweiligen Haltergröße werden Sensorfolien der genannten Dimensionierungen angeboten. Die erfassten Informationen werden digital über die mitgelieferte Systemsoftware „T-Scan 8.1“ verarbeitet. Hinsichtlich der Funktionsweise des Systems nimmt die Sensorfolie eine wesentliche Aufgabe ein. Sie weist eine Materialstärke von $100 \mu \mathrm{m}$ auf und ist je nach Foliendimension (small/large) mit 1122 bzw. 1370 drucksensitiven Messzellen (Leiterbahnen) bestückt, die in sogenannten rows (Zeilen) und columns (Spalten) angeordnet sind (Kerstein et al. 2006). Die Kraftaufzeichnung erfolgt mit 100 Hertz. Befindet sich die Sensorfolie inzwischen einer antagonistischen Krafteinwirkung, wie es in einem Zahn-Zahn-Kontakt der Fall ist, kommt es zu einem Spannungsabfall in den betroffenen Messzellen. Jede Messzelle ist einem Übertragungspol des Handstücks zugeordnet, der den Spannungsabfall registriert und an die Systemsoftware zur Verarbeitung weiterleitet. Zusätzlich verfügt das Handstück über KurzKommando-Bedienelemente, die eine Fernsteuerung des Programms ermöglichen. Aufzeichnungsbeginn und -ende sind so ohne Ablegen des Handstücks durchführbar. Wurde ein vollständiger Datensatz im Sinne eines Bissregistrates ermittelt, kann dieser über verschiedene Menüoptionen analysiert werden (Abb. 10). 


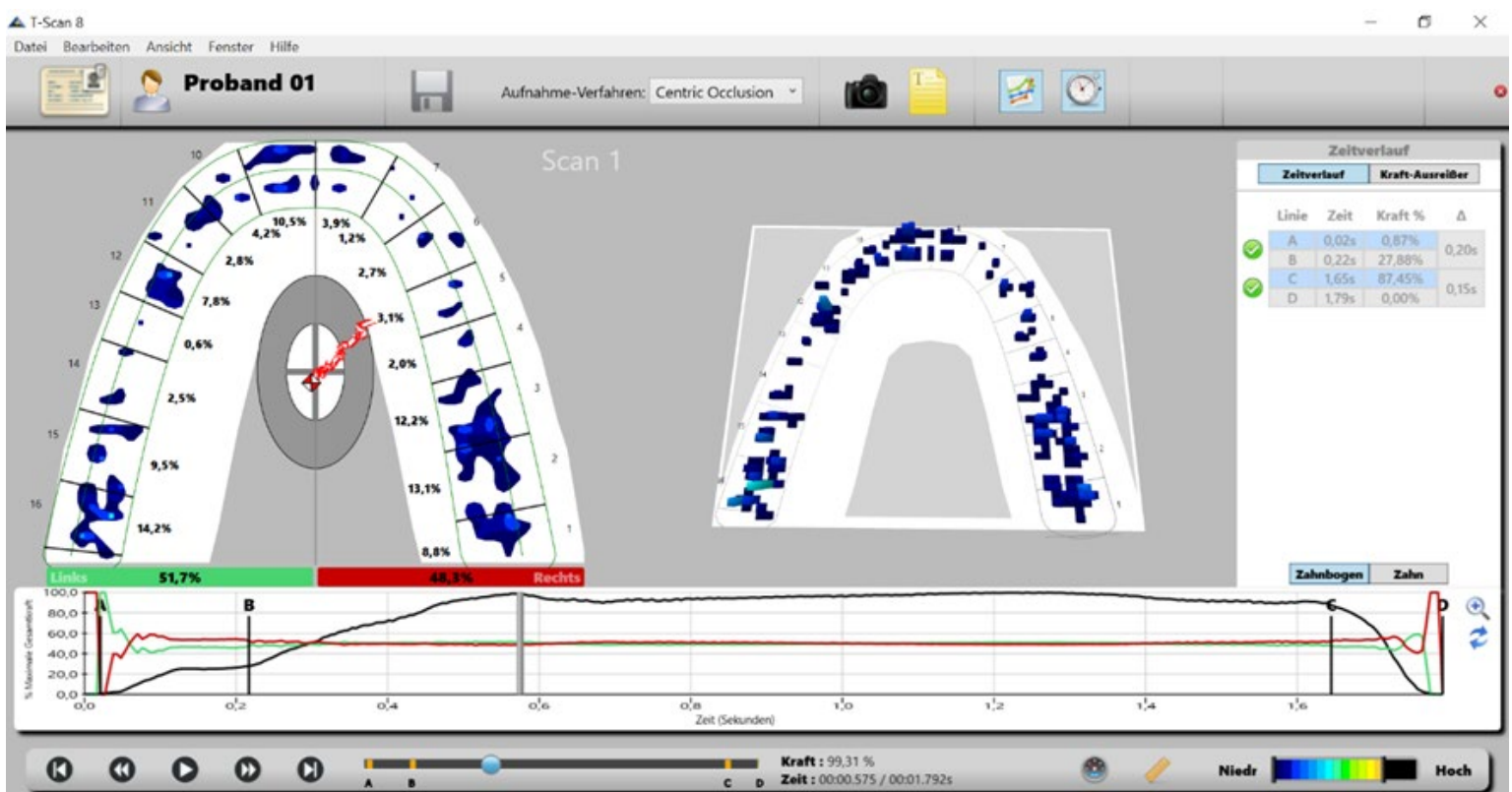

Abbildung 10: T-Scan-8.1-Analyse. Analyseoberfläche für die statische Okklusion der Software T-Scan 8.1 (Tekscan, South Boston, USA)

Das System liefert Auskünfte über die Okklusionskontakte unter Zuordnung der beteiligten Zahnoberflächen. Die genaue zeitliche Abfolge der Kontaktentwicklung, wie auch die prozentuale Beteiligung an der Gesamtkaukraft, können abgebildet werden. Vor der Anwendung des Gerätes besteht die Möglichkeit, den virtualisierten Zahnbogen mittelwertig über die Zahnbreite des ersten Oberkieferinzisiven zu individualisieren. Nach Angabe einer bestimmten Frontzahnbreite berechnet das System die zu erwartenden Zahnbreiten der übrigen Zähne des Oberkieferzahnbogens. Zusätzlich besteht die Möglichkeit, sämtliche Zahnbreiten für die/den jeweilige/-n Probandin/-en einzugeben. Das erneuerte Auswertungsprogramm erlaubt eine Echtzeiterfassung der Messung und macht eine Videografie möglich. Das Programm liefert Auskünfte über die Kontaktflächenverteilung via Balkendiagrammen und kann dem aufgezeichneten Biss dezidierte Zeitintervallpunkte mit individuellen Kraftschwerpunkten zuordnen. Die erhobenen Kraftwerte werden in Relation zur aufgewandten Gesamtkraft dargestellt, die wiederum nicht in einer absoluten Größe ermittelbar ist. Topografisch können die Kontaktflächen, durch vorhergegangene Vermessung der jeweiligen Zahnbreiten des Oberkiefers, nach Eingabe in das Programm zugeordnet werden. Jede Messung besitzt ein zeitliches Auflösungsvermögen von einer Millisekunde und kann einzeln oder mit einer weiteren Aufnahme im geteilten Bildschirmmodus analysiert werden. Das System stellt die erhobenen Okklusionskontakte sowohl als Säulendiagramm also auch in 2-D auf einem virtualisierten Zahnbogen dar. 
Die unterschiedlich hoch abgebildeten Säulen geben Auskunft über die prozentuale Beteiligung an der gesamt gemessenen Kaukraft. Beide Schemata verfügen zusätzlich über eine Spektralfarben-Codierung zur farblichen Abstufung der relativen Kontaktpunktstärke. Blau markierte Areale weisen hierbei auf die relativ geringste, rot markierte auf die relativ größte Kraft hin. Neben der 2- und 3-D-Darstellung der Bisssituation wird ein Kraft-Zeit-Diagramm (Abb. 10) dargestellt, das über die Gesamtdauer der Aufzeichnung sowohl vorwärts als auch rückwärts sowie in verschiedenen Geschwindigkeiten abgefahren werden kann. Das Diagramm liefert drei verschiedene, jedoch miteinander korrespondierende Graphen, die sich aus der relativen Kraft (yAchse) und der absoluten Zeit (x-Achse) bilden. Der schwarze Graph gibt die prozentuale Gesamtkraft zum jeweiligen Zeitpunkt an. Der in Rot abgebildete Graph kennzeichnet parallel dazu die prozentuale Beteiligung der rechtsseitigen Okklusion, der grüne Graph die der linksseitigen. Eine symmetrische Kaukraftverteilung der rechten und linken Seite liegt vor, sobald sich der grüne und der rote Graph bei 50 \% schneiden und aufeinander weiterlaufen.

Die klinische Anwendung der relativ dünnen Folie zeigte, dass vor allem tiefe Bisse mit starken Frontzahnüberbissen zu einer Verwindung der Folie im anterioren Zahnsegment führten und dadurch teilweise keine zuverlässige Zuordnung möglich war. Diverse Studien berichteten dazu ihre Erfahrungen unter besonderer Berücksichtigung prothetisch und implantologisch versorgter Patienten (Afrashtehfar und Qadeer 2016; Garg 2007; Kerstein 2008c; Koos et al. 2010; Throckmorton et al. 2009).

Hinsichtlich der Genauigkeit und Reproduzierbarkeit von Kontaktarealen, die durch das TScan-System ermittelt wurden, führte auch Freesmeyer (1991) eine Studie durch. Der T-Scan sollte mit der Darstellung einer üblichen Okklusionsfolie verglichen werden. 15 vollbezahnte Probanden/innen ohne prothetische Versorgungen wurden hierzu untersucht. Die Ergebnisse zeigten, dass sich die erhobenen Kontaktareale lediglich mit einer Übereinstimmung von 50 \% in Hinblick auf Topografie und Häufigkeit glichen. Darüber hinaus konnte lediglich eine Reproduzierbarkeit mit 70 \% Übereinstimmung festgestellt werden, die Freesmeyer für unbefriedigend befand (Freesmeyer 1991).

Die Arbeitsgruppe um Garrido Garcia et al. (1997) verwendete die nächste Generation des TScans in einer Untersuchung mit 18 erwachsenen Probanden/-innen der Altersspanne 28 bis 50 Jahre. Als Einschlusskriterium wurde die physiologische Vollbezahnung vorausgesetzt. Die Arbeitsgruppe registrierte bei jedem/-er Probanden/-in viermal den Schlussbiss unter maximaler Kraftentfaltung. Die Ergebnisse zeigten eine höhere Varianz zwischen den verschiedenen Probanden/-innen als bei den Messungen des/der jeweiligen Probanden/-in. Zu 90,3 \% stimmten die vier erhobenen digitalen Bissregistrate der Probanden/-innen überein. 
Die Arbeitsgruppe bewertete auf Basis dieser Erkenntnisse die Verwendung des T-Scans zur Okklusionserfassung als nützlich. Weiterhin konnte auch in dieser Untersuchung eine deutlich geringere Kontaktpunktanzahl, als in der ursprünglichen Modellform gefordert, festgestellt werden. Die höchste Kontaktpunktanzahl wurde an den zweiten Molaren, die geringste an den unteren mittleren Inzisivi beobachtet (Garrido Garcia et al. 1997).

Eine weitere Studie derselben Arbeitsgruppe fokussierte sich in ihrer Untersuchung besonders auf die Varianz der Kontaktflächenverteilung innerhalb einzelner Probanden/-innen unter Verwendung des T-Scan-Systems (González Sequeros et al. 1997). Hierzu stand dasselbe Probandengut, nämlich 18 Teilnehmer der Altersspanne 28 bis 50 Jahre, zur Verfügung. Genau wie in der vorigen Studie dieser Arbeitsgruppe beschrieben, wurde bei jedem/-er Probanden/-in viermal die maximale Interkuspidation mittels T-Scan aufgezeichnet und ausgewertet. Hierbei fanden die dritten Molaren keine Berücksichtigung. Die Arbeitsgruppe konnte zeigen, dass die Kontaktflächendarstellung innerhalb aller vier registrierten Bisse, bis auf drei Zähne keine signifikanten Unterschiede aufwies. Ebenfalls wie zuvor festgestellt, fanden sich auch in dieser Untersuchung die meisten Kontakte auf den zweiten Molaren. Die Arbeitsgruppe kam erneut zu dem Schluss, dass sich das T-Scan-System als einfaches Verfahren zur reproduzierbaren Okklusionsanalyse eignet (González Sequeros et al. 1997).

Eine weitere Studie mit höherer Probandenzahl wurde durch Maness und Podoloff (1989) durchgeführt. 93 adulte, vollbezahnte Probanden/-innen wurden einmalig mit dem T-Scan-System untersucht. Der Versuchsaufbau sah die Registrierung der habituellen Interkuspidation in aufrechter Sitzposition vor. Nach Auswertung aller Daten konnte festgehalten werden, dass die registrierte Kontaktflächenverteilung mittels T-Scan - hinsichtlich Reproduzierbarkeit und Genauigkeit etablierter Verfahren - gleichwertige Ergebnisse lieferte. Im Gegensatz zu bereits beschriebenen Studien konnte hier eine symmetrische Kontaktpunktverteilung im untersuchten Probandengut festgestellt werden (Maness und Podoloff 1989).

Die Arbeitsgemeinschaft um Koos (2010) untersuchte ebenfalls die Messgenauigkeit und Reliabilität des T-Scan-Systems, genauer des T-Scan III. Motivation für diese Untersuchung war die Tatsache, dass die gegenwärtige Okklusionsanalyse im klinischen Verfahren nach wie vor auf die Verwendung von markierenden Folien reduziert würde. Koos et al. (2010) formulierten ebenfalls klar, dass die etablierten Verfahren - im Vergleich zu digitalen Messverfahren - nicht in der Lage seien, eine genaue, temporär aufgelöste Kraftverteilung sowie Kraftentwicklung darzustellen. Auf Basis dieser Forderung stellte der Arbeitskreis eine Studienpopulation aus 42 Probanden/-innen, bestehend aus 23 männlichen und 19 weiblichen Teilnehmern, zusammen. Das mediane Alter der Untersuchten lag bei 26 Jahren. 
Die Untersucher führten je Proband/-in insgesamt sechs Messaufzeichnungen mit zwei Folien durch. Insgesamt wurden so 30 Kauzyklen aufgezeichnet. Die Auswertung der Ergebnisse widmete sich besonders der Messgenauigkeit und Reliabilität des Verfahrens sowie dem Einfluss des Folienwechsels auf die Untersuchung. Koos et al. (2010) konnten eine deutliche Kraftverteilungsspanne der jeweiligen Zähne von 0 bis $41 \%$ feststellen, wobei der mittlere Messwert pro Zahn 6,9 \% der entwickelten Gesamtkraft betrug. Es konnten keine statistisch signifikanten Einflüsse durch Folienwechsel und Wiederholungsmessungen auf den erhobenen Messwert festgestellt werden. Die Arbeitsgruppe sah aus Gründen der Differenzierbarkeit der Kraftentfaltung pro Zahn eine deutliche Überlegenheit dieser Methode gegenüber anderen Verfahren. Als Ergänzung zur Routinediagnostik, und besonders mit adjuvanter Verwendung markierender Folien zur intraoralen Navigation, sei das Verfahren ideal (Koos et al. 2010).

Basierend auf dieser positiven Evaluation des T-Scan-Systems, wurde von Koos et al. (2012) eine weitere Studie zur Untersuchung des temporären Kraftaufbaus in Assoziation mit der Kontaktflächendistribution publiziert. Zielsetzung darin war es, sich der bis dato schwachen Datenlage hinsichtlich der detaillierten Analyse des genauen zeitlichen Ablaufs von Kraftaufbau und der Kontaktpunktverteilung in der Okklusion anzunehmen. Die Studienpopulation setzte sich aus 38 Probanden/-innen zusammen, die sich 190 registrierten Kauzyklen mit je fünf Wiederholungen unterzogen. Evaluiert wurden die initialen 0,15 Sekunden nach dem primär registrierten Antagonistenkontakt. Die weitere Registrierung erfolgte in 0,01-Sekunden-Schritten unter Berücksichtigung der in zuvor beschriebener Studie formulierten Messgenauigkeit des verwendeten Systems. Die Arbeitsgruppe konnte bei $44 \%$ der Probanden/-innen zum Zeitpunkt t1 einen initialen Molarenkontakt registrieren. Weitere $40 \%$ wiesen jedoch zum selben Zeitpunkt einen initialen Frontzahnkontakt auf. Lediglich 16 \% zeigten sowohl einen gleichzeitigen Seitenund Frontzahnkontakt. Zum Zeitpunkt t10, also nach 0,1 Sekunden, fand sich die überwiegende Kontaktpunktanzahl auf den Molaren und Prämolaren. Die relative Kaukraftdistribution änderte sich im Zeitverlauf von initial relativ großer Kraft auf den mittleren Inzisiven zu terminal eher posteriorer Lokation. Hinsichtlich der Symmetrie der Kaukraftverteilung konnte ebenfalls eine Seitendifferenz festgestellt werden, die sich über den Verlauf der Messpunkte tendenziell nivellierte. So konnte zum Zeitpunkt t2 eine Seitenverteilung von rechts 57,4 \% zu links 42,6 \% festgestellt werden, die sich zum Zeitpunkt t10 signifikant auf rechts (52,6\%) und links (47,4\%) umverteilte. Die Seitendifferenz verringerte sich damit von t2 zu t10 um 9,6\%. Schlussfolgernd konnte die Arbeitsgruppe feststellen, dass die erhobenen theoretischen Daten einer sich gleichzeitig und gleichmäßig aufbauenden Interkuspidation als Norm widersprechen. 
Weiterhin wurde formuliert, dass die detaillierte Verlaufsbetrachtung den Schluss zulässt, die initialen Inzisivenkontakte möglicherweise als Führungsfunktion anzusehen, um im Anschluss eine kontrollierte Distribution der Kaukräfte nach posterior stattfinden zu lassen (Koos et al. 2012).

Die durch Koos et al. (2010) formulierte Idealisierung der klinischen Okklusionsanalyse unter Verwendung des T-Scans mit markierenden Okklusionsfolien zu Navigationszwecken, wurde bereits durch Kerstein (2008a) im Dental Implantology Journal diskutiert. Hierbei stand allerdings vielmehr die Misskonzeption der Verwendung von markierenden Okklusionsfolien im Vordergrund (Kerstein 2008a). Der Autor kritisierte das nicht wissenschaftlich belegte Auswertungsschema der verschiedenen Kontaktpunktkonfigurationen, das seit mehr als 100 Jahren bestand habe. Der Anwender sei mittels Okklusionsfolien lediglich in der Lage, Vermutungen hinsichtlich der Klassifizierung einer leichten oder starken Kontaktpunktkonfiguration anzustellen (Kerstein 2008a). Weiterhin erwähnt der Autor andere Studien zur Okklusionsanalyse mittels Okklusionsfolie, die keine wissenschaftlichen Beweise für die Aussagekraft des verwendeten Materials hinsichtlich der okklusalen Krafteinwirkung geliefert hätten. Weiterhin erwähnt der Autor die Studien von Halperin et al. (1982) und Schelb et al. (1985), die sich der Okklusionsanalyse mittels -folie gewidmet hatten, ohne wissenschaftliche Beweise für die Aussagekraft des verwendeten Materials hinsichtlich der okklusalen Krafteinwirkung zu liefern. Außerdem verweist Kerstein (2008a) auf die von Millstein (2008) publizierte Arbeit „Know your indicator“, in der festgestellt wird, dass die Literatur keine evidenzbasierten Richtlinien zum Gebrauch von Okklusionsfolien liefert.

Qadeer und Kerstein (Qadeer et al. 2012) konnten im Rahmen einer anderen Untersuchung zeigen, dass die Größe der dargestellten Kontaktareale mittels Okklusionsfolie keine reliable Auskunft über die einwirkende Kraft liefert, was zuvor auch von Carey et al. (2007) in Frage gestellt wurde. Carey et al. (2007) konnten feststellen, dass nur $21 \%$ der untersuchten Kontaktpunktmarken tatsächlich mit der okklusal aufgebrachten Krafteinwirkung korrelierten; $79 \%$ der klinisch feststellbaren und nach üblichen Kriterien ausgewerteten Kontaktpunkte wiesen keine der Kontaktpunktform und -größe gerecht werdende Kraft auf. Anhand eines Fallbeispiels stellte Kerstein (2008a) die Problemstellung unter Zuhilfenahme des T-Scans III vor. Eine Patientin zeigte sich im Zustand post insertionem einer Brückenversorgung über Monate mit schmerzender Okklusion. Zahlreiche Justierungsversuche unter Zuhilfenahme markierender Okklusionsfolien wurden unternommen, blieben jedoch ohne Erfolg. Die Verwendung des T-Scans III zeigte einen Kontaktpunkt im posterioren Brückensegment mit einer 48\%igen Kraftaufnahme der Maximalkraft. Somit lag also annähernd die Hälfte der ausgeübten Kaukraft auf nur einem Zahn. 
Die parallele Verwendung einer markierenden Okklusionsfolie lieferte unter fokussierter Befundung des Kontaktpunktmusters einen Kontaktpunkt, der kleiner als sämtliche Kontakte der restlichen Versorgung war (Kerstein 2008a). Kerstein bezeichnete den Informationsgehalt digitaler Messmethoden als essenziell und für die zeitgerechte Patientenbehandlung unabdingbar (Kerstein 2008a, 2001).

\subsubsection{Dental Prescale Occluzer}

Ähnlich der Funktionsweise des T-Scan-Systems, arbeitet der aus Japan stammende Dental Prescale Occluzer ebenfalls mit einer drucksensitiven Folie die jedoch mit farbstoffhaltigen Microkapseln arbeitet (Kinoshita et al. 1982). Die verwendeten Folienträger bieten ein Auflösungsvermögen von über 2000 Messpunkten pro $\mathrm{mm}^{2}$ (Hidaka et al. 1999; Matsui et al. 1996; Suzuki et al. 1997; Suzuki et al. 1994). Die Arbeitsgruppe um Suzuki (1994) konnte jedoch feststellen, dass häufig höhere Kräfte gemessen als tatsächlich appliziert wurden. Hinsichtlich der Reproduzierbarkeit der Ergebnisse eignet sich der Dental Prescale Occlužer dennoch gut zu Verlaufskontrollen und war in Japan Gegenstand zahlreicher kieferorthopädischer Untersuchungen (Alkan et al. 2008; Alkan et al. 2006; Aras et al. 2009; Iwase et al. 2006; Kinoshita et al. 1982; Sodeyama et al. 1998; Yamamura et al. 2005).

\subsubsection{GEDAS}

Das „Greifswald Digital Analyzing System“ (GEDAS) wurde an der Universität Greifswald etabliert. Hierbei wird ein Silikonregistrat mittels Dokumentenscanner in ein digitales Format überführt. Die Silikonbisse werden sowohl durchleuchtend als auch aufleuchtend eingescannt. Sehr enge okklusale Kontaktbeziehungen weisen im Durchleuchtungsscan hierbei helle Areale auf. Der Auflicht-Scan ermöglicht ein weiteres Bild mit exakten Impressionsstrukturen. Anschließend können beide Bilder überlagert werden und die hellen Areale den Zahnimpressionen zugeordnet werden. Essenziell für die Durchführung des Verfahrens ist die Festlegung eines Helligkeitsschwellenwertes. Im Rahmen der Pilotstudie von Hützen et al. (2006) wurde das Vorliegen eines Kontaktpunktes ab einer Silikonschichtstärke von $\leq 20 \mu \mathrm{m}$ festgelegt. Kontaktflächen, die eine höhere oder gleiche Helligkeit aufwiesen, wurden als echte Kontakte bewertet und konnten für weitere qualitative und quantitative Untersuchungen durch ihre korrekte Lokalisation und Dimension auf den Zahnflächen weiterverarbeitet werden (Hützen et al. 2006). Hinsichtlich der Reliabilität der erhobenen Daten konnte eine gute Übereinstimmung der Analyseergebnisse erzielt werden (Hützen et al. 2006). Im Rahmen der SHIP-Studie fand GEDAS in den Jahren von 2002 bis 2006 an 2.597 Probanden/-innen in einer Altersspanne von 23 bis 86 Jahre Anwendung. 
Es konnte festgestellt werden, dass sich sowohl auf der rechten als auch linken Kieferhälfte annähernd gleich viele Zähne in antagonistischem Kontakt befanden (rechts 8,4, links 8,3 Zähne). Die untersuchten herausnehmbaren und festsitzenden Restaurationen wiesen mit hohem Prozentsatz keine antagonistische Kontaktbeziehung im Sinne des veranschlagten Schwellenwertes auf (Oberkiefer: 41 \%, Unterkiefer: 39 \%) (Hützen et al. 2007). 


\subsection{Fragestellung}

Eine möglichst präzise und reproduzierbare Aufzeichnung der statischen und dynamischen Okklusion ist elementarer Bestandteil der diagnostischen Prozedere in allen Fachdisziplinen der Zahnheilkunde. Die etablierten Methoden zur Visualisierung und Analyse von Okklusionsmustern zeigen deutliche Limitationen in der klinischen Anwendung; ein Goldstandard existiert nicht. Trotzdem finden die vorhandenen Prozedere zur Okklusionsdiagnostik breite Anwendung und werden beispielsweise zur Ableitung von funktionell-restaurativen Therapien als entscheidende Parameter angewendet.

In all diesen diagnostischen und therapeutischen Strategien werden die statische und dynamische Okklusion traditionell als konstant und reproduzierbar angesehen, Abweichungen im Rahmen zahnärztlicher Maßnahmen werden dementsprechend häufig auf zahntechnische und zahnärztliche Fehler zurückgeführt. Die vorliegende Untersuchung sollte klären, ob die Okklusionsmuster und die Methoden zur Okklusionsaufzeichnung bei funktionsgesunden Probanden/-innen tatsächlich konstant und reproduzierbar sind, oder ob die dynamische und statische Okklusion beispielweise tageszeitlichen Schwankungen unterliegt. Hierzu kamen verschiedene Methoden zur Okklusionsdiagnostik (Okklusionserhebung mittels markierender Okklusionsfolien, Kontaktfolien, Wachsregistrate, Silikonregistrate und Sensorfolienregistrate) zum Einsatz, um diese auch untereinander deskriptiv zu vergleichen. 


\section{Material und Methode}

\subsection{Material}

\subsubsection{Digitale Okklusionsdiagnostik}

Zur digitalen Messung der Okklusion wurde das System T-Scan III (Tekscan, South Boston, USA) unter Verwendung eines Dell Latitude E5430 Laptops (Dell Inc., Texas, USA) verwendet (Abb.11). Zur möglichst exakten Darstellung wurde vor der ersten Registrierung jeder Oberkieferzahn der Probanden/-innen in seiner mesio-distalen Breite vermessen (Abb. 12 a) und in der Software T-Scan 8.1 (Tekscan, South Boston, USA) eingegeben (Abb. 11). Zusätzliche Individualmerkmale wie etwaige Lückensituationen, fehlende Zähne oder vorhandene Implantate, wie auch prothetische oder konservierende Restaurationen jeglicher Art, wurden ebenfalls in der Software hinterlegt. Die Vermessung erfolgte intraoral mittels Schiebelehre Münchner Modell (Dentaurum, Ispringen, Deutschland) (Abb. 12 a \& b). Anschließend wurde die transversale Breite des Oberkieferzahnbogens von der breitesten Stelle der bukkalen Molarenfläche zur korrespondierenden Gegenseite gemessen (Abb. 12 b), um die korrekte Dimensionierung von Sensorfolien und -halterung (Tekscan, South Boston, USA) zu ermitteln. Nach erfolgter Vermessung wurden die passende Sensorfolie (Abb. 13 a) sowie der korrespondierende Sensorfolienhalter (Abb. 13 b) in das T-Scan-Handstück eingespannt.

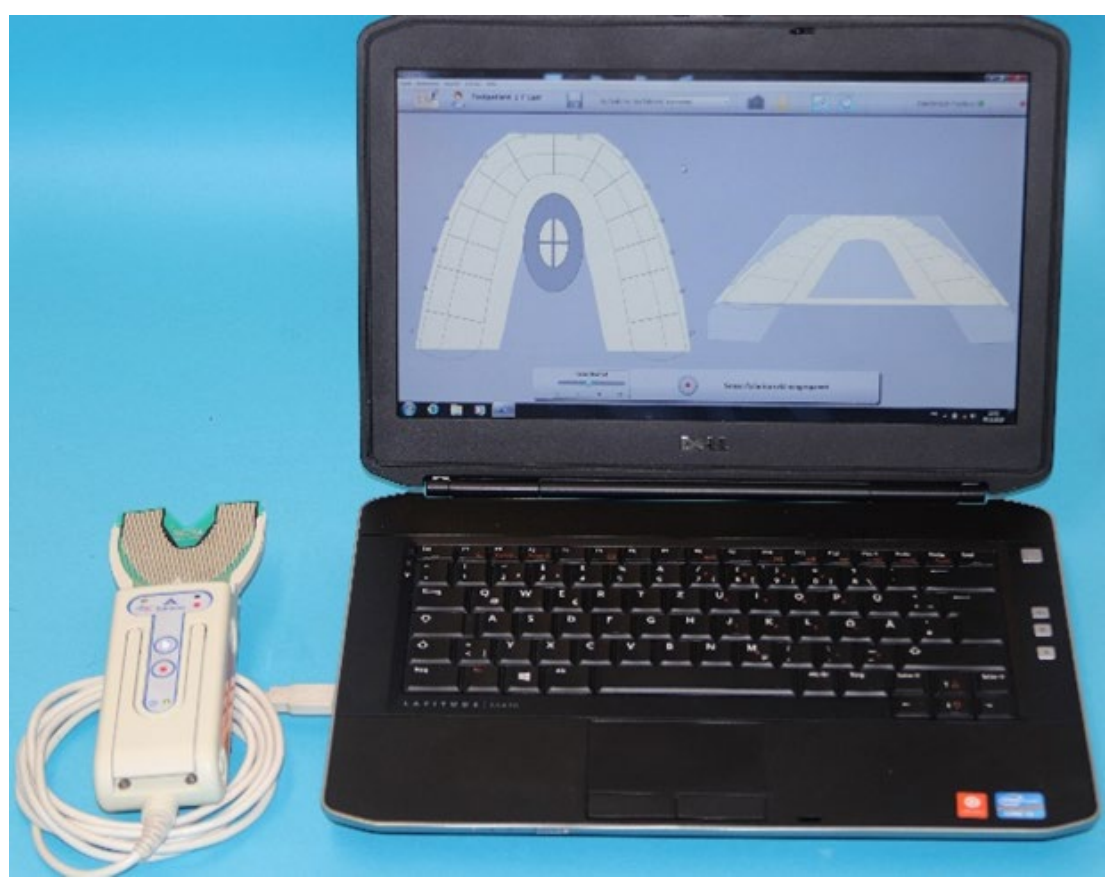

Abbildung 11: Anwendungseinheit des T-Scan-III-Systems. Bestehend aus Sensorhandstück, Software (Tekscan, South Boston, USA) und Laptop Dell Latitude E5430 (Dell Inc., Texas, USA). 


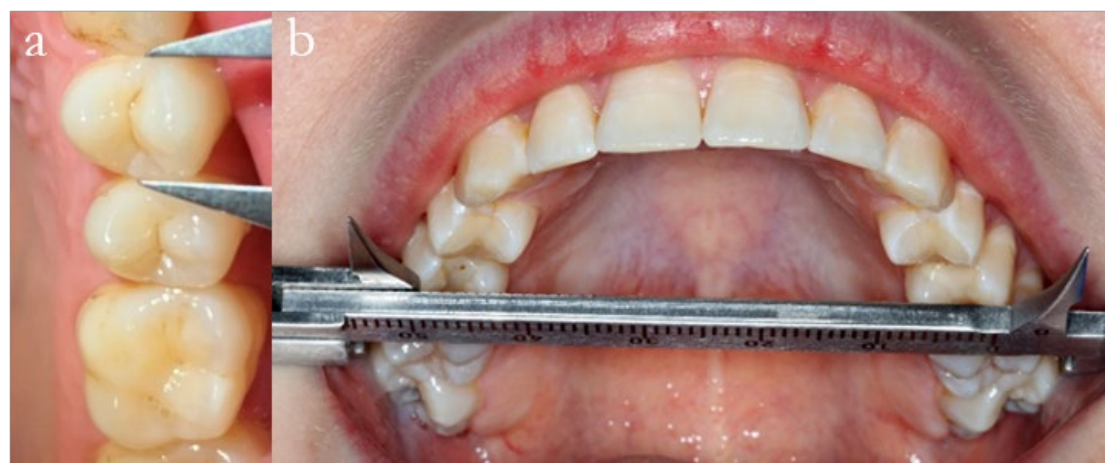

Abbildung 12: Zahnbogenvermessung. Vermessung der Zähne in mesio-distaler Breite (a) und des Zahnbogens (b).

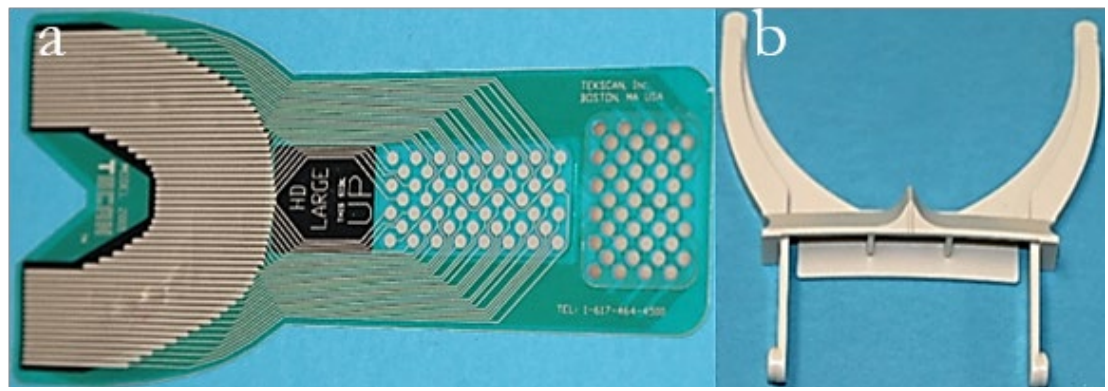

Abbildung 13: Sensorfolie und Sensorfolienhalter. Die primäre Registrierungseinheit bestehend aus Sensorfolie (a) und Sensorfolienhalter (b).

\subsubsection{Okklusionsfolie}

Zum Einsatz kam die Hanel Okklusionsfolie $12 \mu \mathrm{m}$ (Coltène/Whaledent GmbH \& Co. KG, Langenau, Deutschland) mit einer Breite von $22 \mathrm{~mm}$ und einseitiger schwarzer bzw. roter Färbung. Die schwarz markierende Folie wurde für die Erhebung der statischen-, die rot markierende Folie für die Erhebung der dynamischen Okklusion verwendet. Die Folien wurden in Folienpinzetten (Aesculap AG, Tuttlingen, Deutschland) eingespannt.

\subsubsection{Kontaktfolie}

Verwendet wurde die Hanel Shimstock-Folie $8 \mu \mathrm{m}$ (Coltène/Whaledent GmbH \& Co. KG, Langenau, Deutschland). Sie ist in 5-Meter-Rollen der Breite $8 \mathrm{~mm}$ erhältlich und wurde bedarfsgerecht zugeschnitten. Zur intraoralen Anwendung werden die Folienzuschnitte in eine Folienpinzette (Aesculap AG, Tuttlingen, Deutschland) eingespannt. 


\subsubsection{Silikon-Bissregistrat}

Das verwendete Silikon war Voco Registrado X-tra (Voco GmbH, Cuxhaven, Deutschland). Das Produkt ist in einer Zwei-Kammern-Kartusche erhältlich und wird mittels Kartuschenpistole über einen Stempel ausgetrieben. Über eine aufschraubbare Mischkanüle an der Kartuschenöffnung werden beide Silikonkomponenten bei Stempeldruck ausgetrieben und über die Kanülenspule homogenisiert.

\subsubsection{Wachs-Bissregistrat}

Zum Einsatz kam HS-Modellierwachs (Henry Schein INC., Melville, NY 11747, USA) in einer 175 x $80 \mathrm{~mm}$ messenden und 1,25 mm starken Wachsplatte. Das Material setzt sich aus einer Mischung von Kohlenwasserstoff- und Paraffinwachsen zusammen. Der Erstarrungspunkt liegt bei $55^{\circ} \mathrm{C}$. Da der Schmelzpunkt des Wachses zwischen 54 und $61^{\circ} \mathrm{C}$ liegt, wurde ein Wasserbad (Julabo TW2, Julabo GmbH, Seelbach, Deutschland) mit niedrigerer Temperaturkalibrierung $\left(42^{\circ} \mathrm{C}\right)$ zur Erwärmung des Materials verwendet.

\subsection{Methode}

\subsubsection{Probanden/-innen}

Für die vorliegende Studie wurden 19 freiwillige Probandinnen und Probanden davon 14 Frauen und fünf Männer untersucht. Die Untersuchung wurde von der Ethikkommission genehmigt (Nummer Antrag 18/9/17). Jede/-r Proband/-in wurde im Rahmen eines Aufklärungsgesprächs über die Studiendurchführung aufgeklärt und willigte in vollem Umfang mündlich und schriftlich in die Studienteilnahme ein. Einschlusskriterium für die Teilnahme war neben dem vollendeten 18. Lebensjahr ein funktionsgesundes Kausystem, das im Rahmen des CMDKurzbefundes (Abb. 14) nach Ahlers und Jakstat (2015) und dem klinischen Funktionsstatus der deutschen Gesellschaft für Funktionsdiagnostik und -therapie (DGFDT) in der DGZMK überprüft wurde. 


\section{CMD-Kurzbefund dentaConcept}

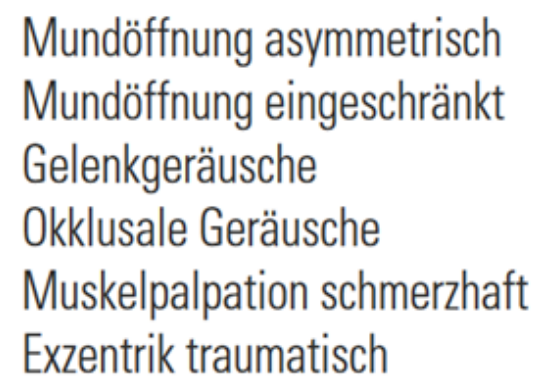

Mundöffnung asymmetrisch

Mundöffnung eingeschränkt

Gelenkgeräusche

Okklusale Geräusche

Muskelpalpation schmerzhaft

Exzentrik traumatisch

\section{CMD \\ O unwahrscheinlich $(\leq 1)$ \\ O wahrscheinlich $\quad(\geq 2)$}

Abbildung 14: CMD-Kurzbefund. Nach Ahlers und Jakstat (2015). Die Verwendung erfolgt mit freundlicher Genehmigung von PD Dr. M. Oliver Ahlers, Copyright (C) dentaConcept.

Zum Ausschluss der Studienteilnahme führten Funktionsstörungen des craniomandibulären Systems. Darüber hinaus wurden Probanden/-innen mit allgemein-zahnärztlichem Behandlungsbedarf (Karies, Parodontitis etc.) ebenfalls von der Studie ausgeschlossen. Die Befundung genannter Ausschlusskriterien zog die Einleitung notwendiger Therapiemaßnahmen nach sich. Patienten mit umfangreichen prothetischen Versorgungen im Sinne eines herausnehmbaren Zahnersatzes und Patienten mit Brücken wurden von der Untersuchung ausgeschlossen. Probanden/-innen, welche die Einverständniserklärung nicht unterzeichnet haben oder nicht volljährig oder entscheidungsfähig waren, wurden ebenfalls nicht in die Studie aufgenommen.

\subsubsection{Versuchsaufbau und Durchführung}

Alle Methoden zur Okklusionsaufzeichnung kamen für jeden Patienten mehrmals und unter strenger Einhaltung des vorgegebenen Zeitplans zum Einsatz. Der Versuchsaufbau sah die Registrierung der habituellen Interkuspidation jedes/-r Probanden/-in an drei Tageszeiten, nämlich um 9-, 12- und 16 Uhr, mit einer Wiederholung des Prozederes in 14-tägigem Abstand vor. Die Okklusion wurde zu den jeweiligen Zeitpunkten digital mittels T-Scan-III-System (statisch und dynamisch) sowie konventionell anhand von Bissregistraten mit Registrado X-tra und HSModellierwachs (statisch), Shimstock-Okklusionsfolie (statisch) und Okklusionsfolie (schwarz: statisch, rot: dynamisch) erhoben (siehe Tab. 1 \& 2). Alle klinischen Messungen wurden durch einen approbierten Zahnarzt durchgeführt. 
Tabelle 1: Übersicht der Registrierverfahren

\begin{tabular}{|c|c|}
\hline Verfahren & Okklusionstyp \\
\hline \multicolumn{2}{|c|}{ Digital } \\
\hline T-Scan III & statische Okklusion \\
\hline T-Scan III & dynamische Okklusion n. links \\
\hline T-Scan III & dynamische Okklusion n. rechts \\
\hline \multicolumn{2}{|c|}{ Konventionell } \\
\hline Registrado X-tra & statische Okklusion \\
\hline HS-Modellierwachs & statische Okklusion \\
\hline Shimstock-Folie $8 \mu \mathrm{m}$ & statische Okklusion \\
\hline Okklusionsfolie $12 \mu \mathrm{m}$ schwarz & statische Okklusion \\
\hline Okklusionsfolie $12 \mu \mathrm{m}$ rot & dynamische Okklusion n. links \\
\hline Okklusionsfolie $12 \mu \mathrm{m}$ rot & dynamische Okklusion n. rechts \\
\hline
\end{tabular}

Tabelle 2: Untersuchungsübersicht

\begin{tabular}{|l|l|}
\hline \multicolumn{2}{|c|}{ Untersuchungstag 1 und 2 } \\
\hline Uhrzeit & \multicolumn{1}{|c|}{ Verfahren } \\
\hline \multirow{2}{*}{9 Uhr } & \multicolumn{1}{|c|}{ Digital } \\
\cline { 2 - 3 } & $\begin{array}{l}\text { T-Scan III: stat. Okklusion I } \\
\text { T-Scan III: dyn. Okklusion n. links I } \\
\text { T-Scan III: dyn. Okklusion n. rechts I }\end{array}$ \\
\cline { 2 - 3 } & $\begin{array}{l}\text { Kegistrado X-tra: stat. Okklusion I } \\
\text { HS-Modellierwachs: stat. Okklusion I } \\
\text { Shimstock-Folie 8 } \mu \text { m: stat. Okklusion I } \\
\text { Okklusionsfolie (schwarz): stat. Okklusion I } \\
\text { Okklusionsfolie (rot): dyn. Okklusion li. I }\end{array}$ \\
\hline
\end{tabular}




\begin{tabular}{|c|c|}
\hline \multicolumn{2}{|c|}{ Untersuchungstag 1 und 2} \\
\hline & Okklusionsfolie (rot): dyn. Okklusion re. I \\
\hline \multirow{9}{*}{$12 \mathrm{Uhr}$} & Digital \\
\hline & $\begin{array}{l}\text { T-Scan III: stat. Okklusion II } \\
\text { T-Scan III: dyn. Okklusion n. links II } \\
\text { T-Scan III: dyn. Okklusion n. rechts II }\end{array}$ \\
\hline & Konventionell \\
\hline & Registrado X-tra: stat. Okklusion II \\
\hline & HS-Modellierwachs: stat. Okklusion II \\
\hline & Shimstock-Folie $8 \mu \mathrm{m}$ : stat. Okklusion II \\
\hline & $\begin{array}{l}\text { Okklusionsfolie (schwarz): stat. Okklusion } \\
\text { II }\end{array}$ \\
\hline & Okklusionsfolie (rot): dyn. Okklusion li. II \\
\hline & Okklusionsfolie (rot): dyn. Okklusion re. II \\
\hline \multirow{11}{*}{$16 \mathrm{Uhr}$} & Digital \\
\hline & T-Scan-III: stat. Okklusion III \\
\hline & T-Scan III: dyn. Okklusion n. links III \\
\hline & T-Scan III: dyn. Okklusion n. rechts III \\
\hline & Konventionell \\
\hline & Registrado X-tra: stat. Okklusion III \\
\hline & HS-Modellierwachs: stat. Okklusion III \\
\hline & Shimstock-Folie $8 \mu \mathrm{m}$ : stat. Okklusion III \\
\hline & Okklusionsfolie (schwarz): stat. Okklusion III \\
\hline & Okklusionsfolie (rot): dyn. Okklusion li. III \\
\hline & Okklusionsfolie (rot): dyn. Okklusion re. III \\
\hline
\end{tabular}

Übersicht der durchgeführten Registrierungen je Untersuchungstag und Proband/-in. 


\subsubsection{Digitale Okklusionsanalyse}

Die Messungen erfolgten in aufrechter Sitzposition mit entspannt am Kopfteil angelegtem Kopf (Abb. 15 a). Für die folgenden digitalen Messungen wurden alle Teilnehmer vorab über den Ablauf der Aufnahme wie folgt instruiert: Für die Erhebung der statischen Okklusion initiierte das Kommando „Zu!“ den Zubiss bzw. Kieferschluss. Die Teilnehmer sollten bei diesem Kommando solange zubeißen, bis der maximale Vielpunktkontakt erreicht wurde. Es wurde explizit darauf hingewiesen, nur so fest wie nötig zuzubeißen.

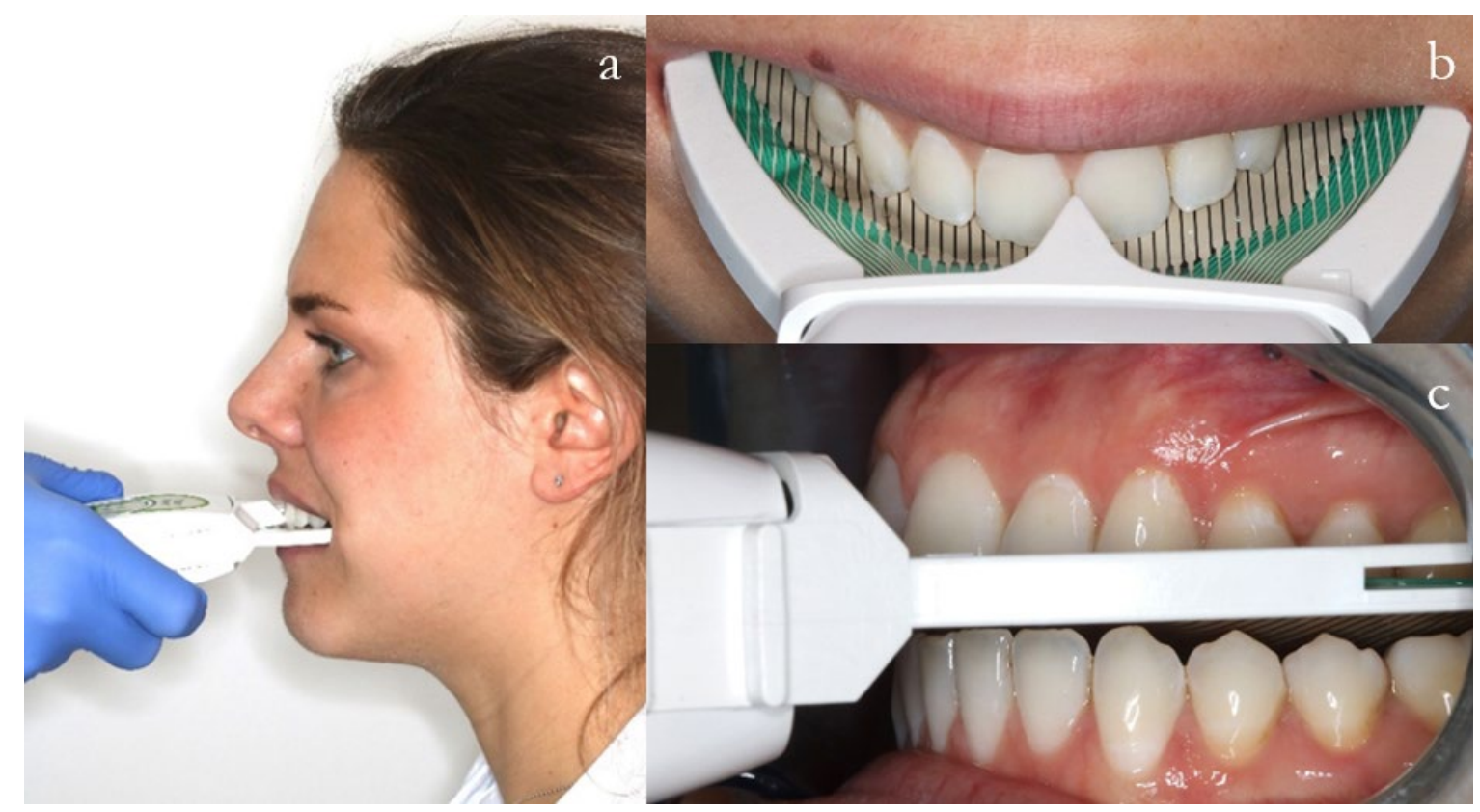

Abbildung 15: Klinische Anwendung des T-Scan III. Die Darstellungen zeigen die korrekte Anwendung des T-Scan III in aufrechter Kopfhaltung (a), parallel zum Okklusionsplanum (b) und während der Registrierung der dynamischen Okklusion nach links (c).

Nach Erreichen der individuellen maximalen Interkuspidation wurde der Patient gebeten, selbstständig den Mund wieder weit zu öffnen, was die Aufnahme terminierte. Für die Erhebung der dynamischen Okklusion nach links und rechts wurde der/die Proband/-in gebeten auf das Kommando „Zu, links!“ bzw. „Zu, rechts!“ zuzubeißen und anschließend eine Exkursionsbewegung des Unterkiefers unter Zahnkontakt zur linken und rechten Seite zu vollführen. Unmittelbar nach Erreichen der Maximalauslenkung des Unterkiefers unter Zahnkontakt zur betroffenen Seite sollte der/die Proband/-in den Mund wieder weit öffnen, was die Aufzeichnung terminierte. Nach abgeschlossener Instruktion wurde mit der digitalen Messung begonnen. Dazu wurde das Handstück nun so zum/-r Probanden/-in geführt, dass der Folienhalter mit der aufgenommenen Sensorfolie parallel zur Okklusionsebene am Oberkieferzahnbogen anlag (Abb. 15 b und c). Hierbei wurde darauf geachtet, dass sich der zentral-anterior angebrachte Inzisivensporn des Folienhalters am Approximalkontakt der beiden Oberkieferinzisiven abstützte (Abb. 15 b). 
Weiterhin wurde berücksichtigt, dass der Ausleger des Folienhalters der linken Seite im selben Abstand zu den Bukkalflächen der Seitenzähne positioniert wird, wie jener der rechten Seite (Abb. 15 b). Vor Beginn der Messung wurde die für den Versuch notwendige Sitzposition eingenommen. Hierbei befindet sich der/die Proband/-in aufrecht im Behandlungsstuhl sitzend, wenn das Sensorfolien-Handstück angehalten wird (Abb. 15 a). Besondere Berücksichtigung wurde der näherungsweisen Oberkieferinklination beigemessen. Bei stark posterior oder anterior inklinierter Maxilla führte eine $90^{\circ}$-Zuführung des Messinstruments nicht zu einer Parallelität der Sensorfolie zur Okklusionsebene. Aus diesem Grund wurde von einer $90^{\circ}$-Zuführung Abstand genommen und die individuelle Okklusionsebene des/der Probanden/-in berücksichtigt, um die Verwindung der Sensorfolie während des Bisses möglichst gering zu halten. Gemäß der Herstellerempfehlung wurde vor der Hauptmessung eine Kalibrierung der individuellen Kaukraft des/der Probanden/-in durchgeführt, im Zuge welcher der/die Proband/-in auf das Kommando „Zu!“ einmalig mit maximaler Kaukraft auf die Folie biss. Nach stattgefundener Kalibrierung konnte die Hauptmessung vorgenommen werden. Der/Die Proband/-in führte jeweils nach dem Kommando „Zu!“ den Bissschluss aus und öffnete nach Erreichen der maximalen Interkuspidation selbstständig. Im Anschluss erfolgte die Messung der dynamischen Okklusion nach links (Abb. 15 c), abschließend die Registrierung der dynamischen Okklusion nach rechts. Zu den verschiedenen Messzeitpunkten (9, 12 und 16 Uhr) wurden also jeweils drei Aufzeichnungen je Proband/-in und Untersuchungstag angefertigt und digital archiviert. Um gleichbleibend reproduzierbare und zuverlässige Ergebnisse zu erhalten, wurde die Sensorfolie nach maximal zehn Aufnahmen gewechselt.

\subsubsection{Silikonregistrat}

Nach der digitalen Erfassung der statischen und dynamischen Okklusion schloss sich die konventionelle Bissregistrierung der statischen Okklusion mittels Quetschbiss mit dem Silikon Registrado X-tra (Voco GmbH, Cuxhaven, Deutschland) an. Wie zuvor wurde auch in diesem Verfahren die aufrechte Sitzposition des Patienten sichergestellt. Unter Berücksichtigung der Verarbeitungszeit von 30 Sekunden wurde der/die Proband/-in gebeten, den Mund zu öffnen. Direkt darauf schloss sich die Applikation einer Silikonspur auf die Unterkieferzahnreihe an (Abb. 16 a). Anschließend wurde der/die Proband/-in über das Kommando „Zu!“ gebeten, den Bissschluss in habitueller Okklusion bis zum maximalen Vielpunktkontakt durchzuführen, und über die verbliebene Verarbeitungszeit hinaus bis zur Aushärtung des Materials nach $40 \mathrm{~s}$ in dieser Situation zu verweilen (Abb. 16 b). 
Nach der durch das Kommando „Auf!“ implizierten weiten Mundöffnung konnte das Bissregistrat nach stattgefundener Abbindephase sicher aus dem geöffneten Patientenmund entnommen und der Tauchdesinfektion MD 520 (Dürr Dental, Bietigheim-Bissingen, Deutschland) zugeführt werden, in der es für 5 Minuten verblieb.

Im Anschluss wurden die Registrate entnommen, luftgetrocknet und zur späteren Analyse und Auswertung dunkel, bei $23^{\circ} \mathrm{C}$ Raumtemperatur gelagert.

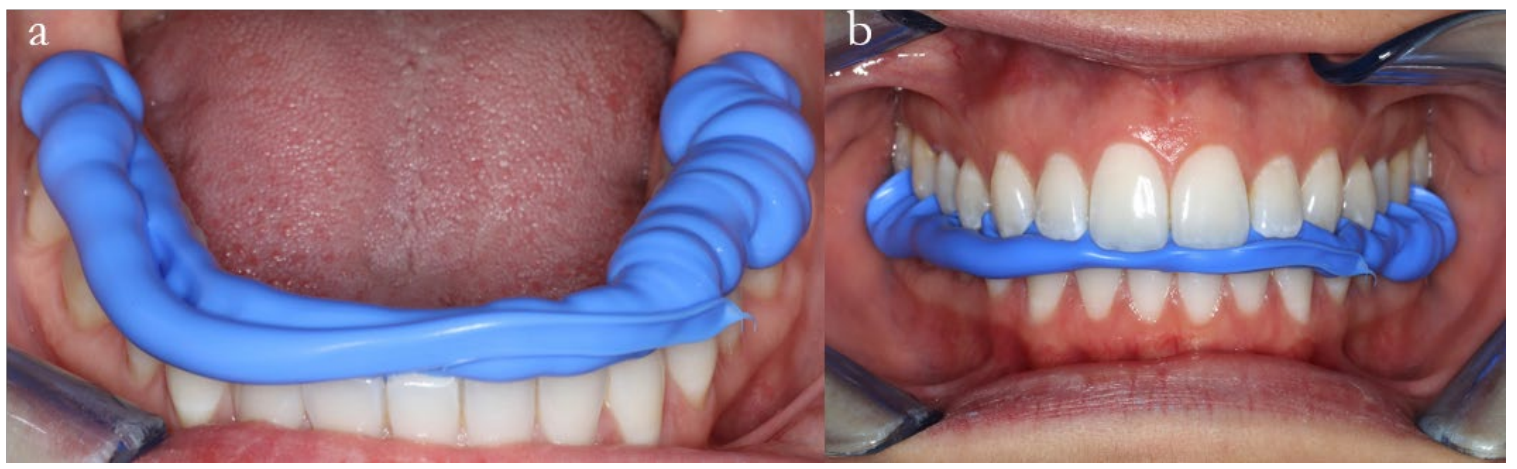

Abbildung 16: Silikonregistrierung. Registrierung der statischen Okklusion mittels Silikon-Quetschbiss (Registrado X-tra): Applikation einer Silikonspur auf den Unterkieferzähnen (a) und Aushärten des Materials nach Einnehmen der habituellen Okklusion durch den Patienten (b).

\subsubsection{Wachsregistrat}

Zur Anwendung kam HS-Modellierwachs (Henry Schein INC., Melville, NY, USA) in einer 175 x $80 \mathrm{~mm}$ messenden, 1,25 mm starken Wachsplatte. Dieses wurde mithilfe eines thermoregulierbaren Wasserbades (Julabo TW2, Julabo GmbH, Seelbach, Deutschland) auf die gewünschte Temperatur von $42^{\circ} \mathrm{C}$ erwärmt. Nach abgeschlossener Aufwärmphase von 5 Minuten, konnte die vorab manuell halbierte Wachsplatte entnommen und - unter Einhaltung der gewünschten, aufrechten Sitzposition - in den geöffneten Patientenmund eingebracht werden (Abb. 17 a). Die Applikation des Wachses erfolgte über leichtes Andrücken am Oberkieferzahnbogen bis zur eigenen Lagestabilität. Aufgrund der schnellen temperaturinduzierten Erstarrung des Materials außerhalb des Wasserbades wurde der Patient umgehend nach Applikation mit dem Kommando „Zu!“ zur habituellen Interkuspidation bis zum maximalen Vielpunktkontakt aufgefordert, um dort zu verharren, bis die Temperaturreduktion zur weiteren Verfestigung des Materials führte (Abb.17 b). Das Kommando „Auf!“ implizierte dann abermals die weite Mundöffnung, wonach das Wachsregistrat aus dem geöffneten Patientenmund entnommen und für 5 Minuten der Tauchdesinfektion zugeführt werden konnte. Der Desinfektion folgten die Lufttrocknung und trockene, dunkle Lagerung (kartoniert) bei $23^{\circ} \mathrm{C}$ Raumtemperatur zur späteren Analyse und Auswertung. 


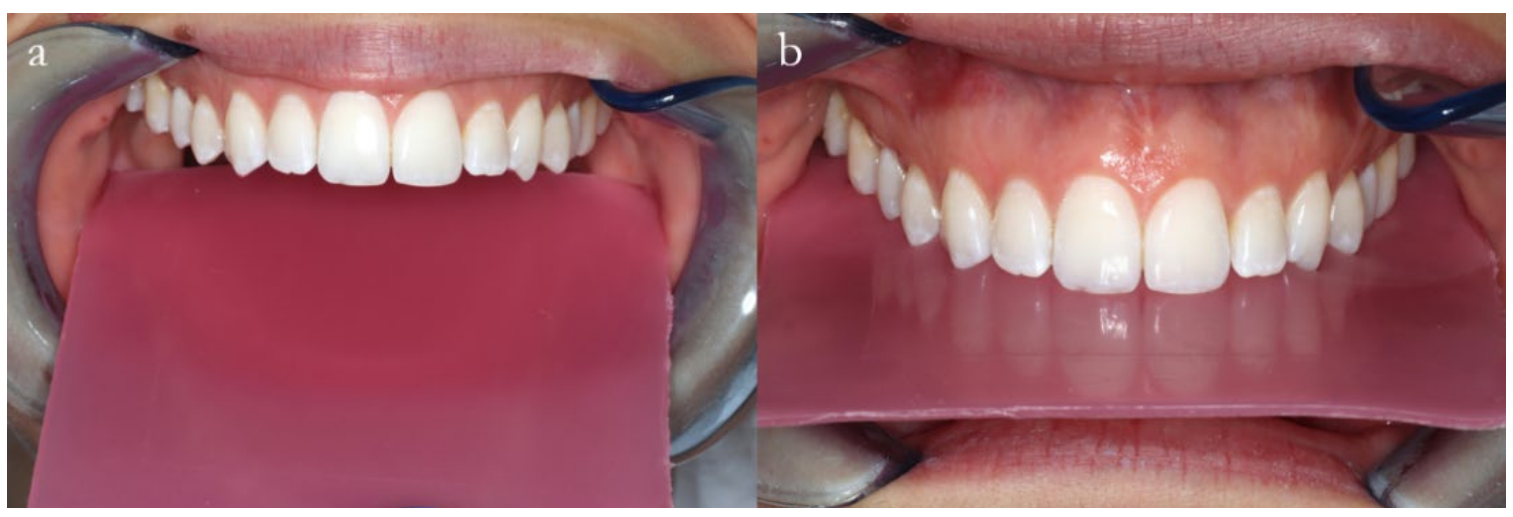

Abbildung 17: Wachsregistrierung. Die Applikation der Wachsplatte (a) mit anschließender Registrierung (b).

\subsubsection{Kontaktfolie}

Für die konventionelle Okklusionsanalyse mittels Kontaktfolie wurde Shimstock-Folie (Coltène/Whaledent AG, Schweiz) verwendet. Hierzu wurden $8 \mu \mathrm{m}$ starke Folienzuschnitte im rechtwinkeligen Maß von 20 x 5 mm in eine Folienpinzette (Aesculap AG, Tuttlingen, Deutschland) (Abb. 18 a) eingespannt. Anschließend wurde die Folienpinzette intraoral am aufrecht sitzenden Teilnehmenden parallel zum Okklusionsplanum zwischen die geöffneten Zahnreihen gehalten. Gleichzeitig wurde die Wange durch die Rückseite der Pinzette abgehalten. Durch das Kommando „Zu!“ führte der/die Proband/-in den Zahnreihenschluss in habitueller Interkuspidation bis zum Erreichen des maximalen Vielpunktkontaktes zur Erhebung der statischen Okklusion durch. In der gewünschten Okklusionsposition wurde auf die im Kontaktareal zu liegen gekommene Folie einer Zugprobe unterzogen, um das Vorhandensein des Zahnantagonismus zu prüfen (Abb. 18 a).

Ließ sich die Folie auf Zug reibungsfrei zwischen den Antagonisten herausziehen, wurde keine direkte Kontaktpunktbeziehung angenommen. Konnte die Folie unter Reibung gerade noch aus dem Kontaktareal gezogen werden, sprach dies für einen regelrechten Kontaktpunkt. Bei vollständiger Arretierung der Folie im Kontaktareal waren starke Kontakte zu befunden. Nach jedem Zugtest öffnete der/die Proband/-in auf das Kommando „Auf!“ den Mund. Dieses Vorgehen wurde für jedes antagonistische Zahnpaar des/der Probanden/-in wiederholt und in einer Befundtabelle (Ottl et al. 2018) (Abb. 18 b) notiert. 

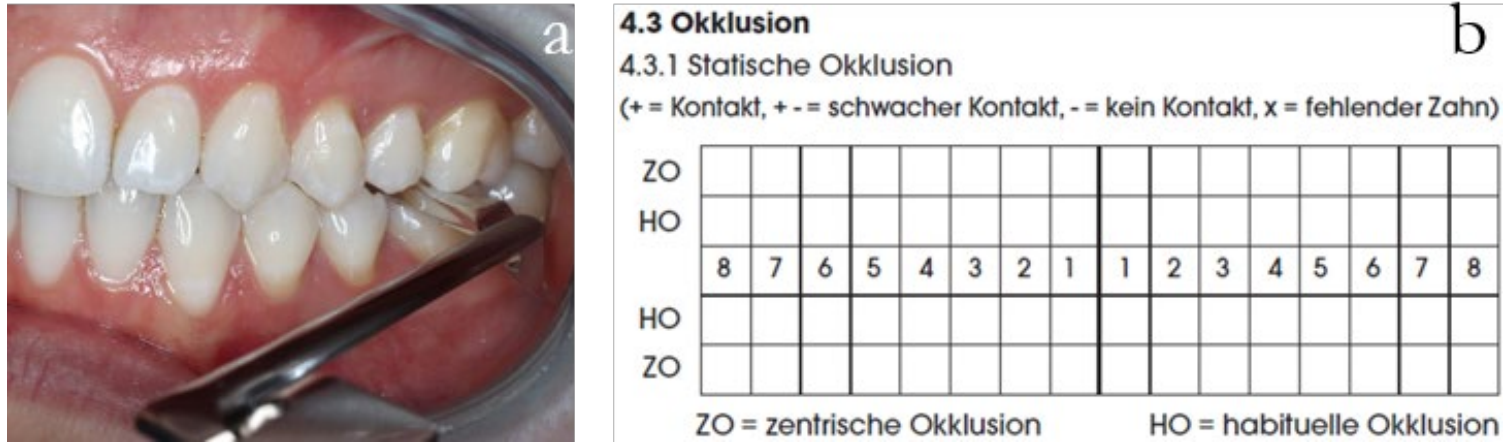

Abbildung 18: Okklusionsbefunde. Die klinische Situation der Kontaktfolien-Registrierung (a) mit dem klinischen Funktionsstatus der DGFDT in der DGZMK (b). Die Verwendung erfolgt mit freundlicher Genehmigung der DGFDT, Copyright (C) Ottl/Ahlers/Lange/Utz/Reiber 2011.

\subsubsection{Okklusionsfolie}

Den Abschluss der Okklusionserfassung stellte die Registrierung der statischen und dynamischen Okklusion mittels Hanel Okklusionsfolie $12 \mu \mathrm{m}$ (Coltène/Whaledent GmbH + Co. KG, Langenau, Deutschland) mit einseitiger roter oder schwarzer Färbung dar. Hierzu wurden Folienzuschnitte der Dimension 22 x 40 mm längs in einer Folienpinzette (Aesculap AG, Tuttlingen, Deutschland) eingespannt. Die Messungen wurden ebenfalls in aufrechter Sitzposition durchgeführt. Zuerst wurden die Zahnreihen im Ober- und Unterkiefer durch einmaliges Aufbeißen auf ein Vlies-Tuch direkt vor der Messung getrocknet. Die Probanden/-innen wurden vorab instruiert, für die Registrierung der statischen Okklusion die habituelle Schlussbisslage bis zum Erreichen eines maximalen Vielpunktkontaktes einzunehmen und anschließend (nach Erreichen der maximalen Interkuspidation) selbstständig den Mund wieder zu öffnen. Da die Okklusionsfolie eingespannt eine rechteckige Konfiguration aufweist, war zur Registrierung des gesamten Zahnbogens ein Umsetzen der Folie nötig. So wurde die Folie nach erstmaliger Registrierung des linken Seitenzahnsegmentes (Abb. 20 b) nach Mundöffnung zum Frontzahnsegment (Abb. 19) (Eckzahn zu Eckzahn) umgesetzt und sofort ein weiterer Schlussbiss in maximaler Interkuspidation registriert. Nach abermaliger Mundöffnung erfolgte die Umsetzung der Folie zum rechten Seitenzahnsegment (Abb. 20 a), wo bei nochmaligem Schlussbiss die maximale Interkuspidation der rechten Seite registriert wurde. Die anschließende Mundöffnung terminierte die Erhebung der statischen Okklusion. Das Kommando „Zu!“ initiierte für jede Registrierung der statischen Okklusion den Schlussbiss in maximaler Interkuspidation. Der/Die Proband/-in terminierte die Messung nach Erreichen des maximalen Vielpunktkontaktes selbstständig. 
Die Farbmarkierungen wurden dann über einen intraoralen Spiegel (RMO Corporate Office/Plant, Denver, USA) unter Zuhilfenahme eines Lippenretraktors (Pelz \& Companion GmbH, Lindenberg, Deutschland) mit einer Spiegelreflexkamera Canon EOS 200D (Canon INC. Tokyo, Japan) fotografiert und am Laptop Dell Latitude E5430 (Dell Inc., Texas, USA) mittels Fotobetrachter des Betriebssystems Windows 732 Bit (Microsoft Corporation, Redmond, USA) in einfacher Vergrößerung Zahn für Zahn analysiert.

Anschließend folgte die Registrierung der dynamischen Okklusion nach links und nach rechts. Hierzu wurden die Zahnreihen wiederum gründlich mit einem Vlies-Tuch gereinigt und durch Aufbiss getrocknet. Nach Einspannen der Okklusionsfolie rot $12 \mu \mathrm{m}$ in die Folienpinzette erfolgte erneut die erste Registrierung im linken Seitenzahngebiet (Abb. 20 d). Die Probanden/innen wurden hierzu vorab instruiert, auf das Kommando „Zu, links!“ und „Zu, rechts!“ den Schlussbiss in maximale Interkuspidation zu vollführen, um anschließend in die maximal mögliche Unterkieferexkursion unter Zahnkontakt nach links, und - über einen Zwischenschritt in der statischen Okklusion - abschließend in die maximal unter Zahnkontakt mögliche Unterkieferexkursion nach rechts zu führen. Nach Erreichen der Maximalbewegung nach rechts führten die Probanden/-innen zurück in die statische Okklusion und terminierten die Registrierung des jeweiligen Zahnsegmentes selbstständig durch weite Mundöffnung, woran sich die Umsetzung der Folie für das Frontzahnsegment anschloss und das Prozedere für das nächste Segment erneut durchgeführt wurde. Terminal erfolgte die Registrierung des rechten Seitenzahnsegmentes (Abb. 20 c) im beschriebenen Ablauf. Nach Registrierung aller Zahnsegmente erfolgten Fotodokumentation, Archivierung und Auswertung der statischen (Abb. 19, 20 a und b, 21 a und b) und dynamischen (Abb. 19, 20 c und d, 21 c und d) Okklusion mittels zuvor definierter Standards. 


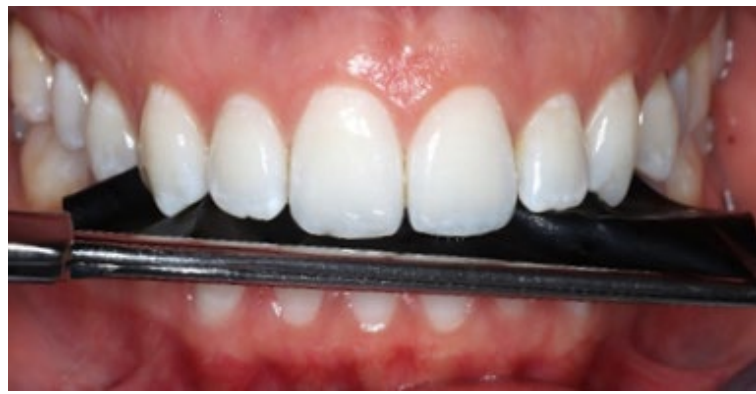

Abbildung 19: Okklusionsfolienregistrierung (statisch). Erhebung der statischen Okklusion der Front mittels Okklusionsfolie $12 \mu \mathrm{m}$.
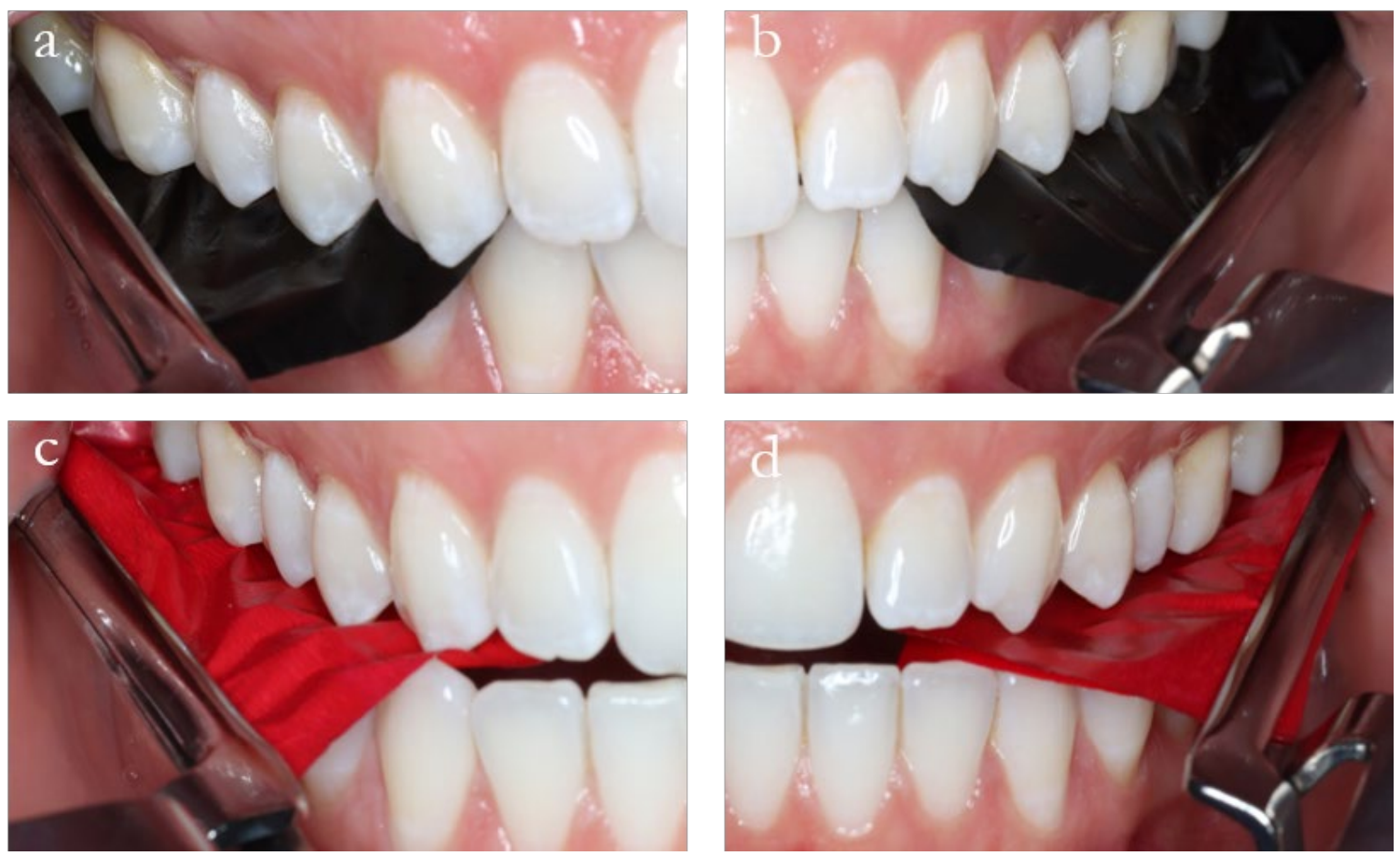

Abbildung 20: Okklusionsfolienregistrierung. Erhebung der statischen (a und b) und dynamischen (c und d) Okklusion mittels Okklusionsfolie $12 \mu \mathrm{m}$. 

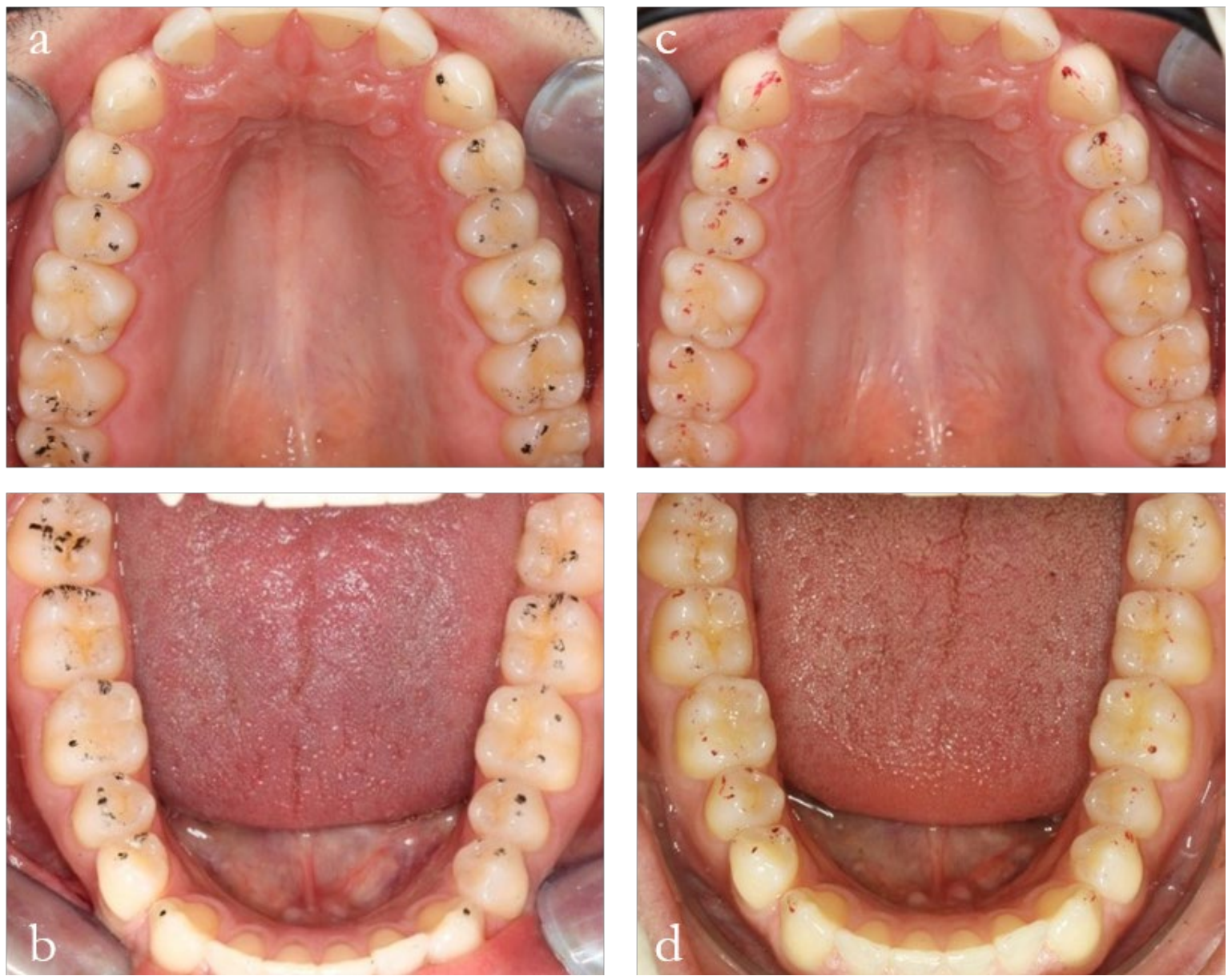

Abbildung 21: Befundungsfoto. Archivierte Auswertungssituation der statischen (a und b) und dynamischen ( $\mathrm{c}$ und d) Okklusionsregistrierung mittels Okklusionsfolie $12 \mu \mathrm{m}$ im Oberkiefer.

\subsubsection{Auswertung}

Die Auswertung sämtlicher Daten wurde durch ein und denselben Untersucher vorgenommen. Alle Untersuchungen fanden unter Einhaltung der Hersteller-Gebrauchsanweisungen statt.

\subsubsection{Auswertung der digitalen Okklusionsanalyse}

Um eine etwaige tageszeitabhängige Varianz in der Okklusion festzustellen, wurde im Rahmen der Auswertung jede Änderung der prozentualen Kaukraftbeteiligung aller Oberkieferzähne an der relativen Gesamtkaufkraft zu einem definierten Zeitpunkt erfasst. Bei Erhebung sämtlicher Okklusionsmuster je Untersuchungszeitraum wurden drei digitale Okklusionsregistrate durchgeführt, nämlich die Analyse der statischen Okklusion, der dynamischen Okklusion rechts und der dynamischen Okklusion links. Je Untersuchungstag wurden demnach neun und in Gesamtheit folglich 18 digitale Okklusionsregistrate für jede Probandin bzw. jeden Probanden analysiert. 
Um eine möglichst reproduzierbare und genaue Analyse der mit dem T-Scan-III-System registrierten Kontaktareale vorzunehmen, wurden die aufgezeichneten Okklusionsmuster aller Probanden/-innen immer mit dem gleichen Auswertungsschema und nach Standardprozedere wie folgt befundet:

Nach Aufruf der jeweiligen Probandenkarte wurde die Justierung des individuell normierten Zeitpunktes der Aufnahme vorgenommen. Hierzu wurde der Zeitpunkt der maximalen Interkuspidation gewählt (Abb. 22).

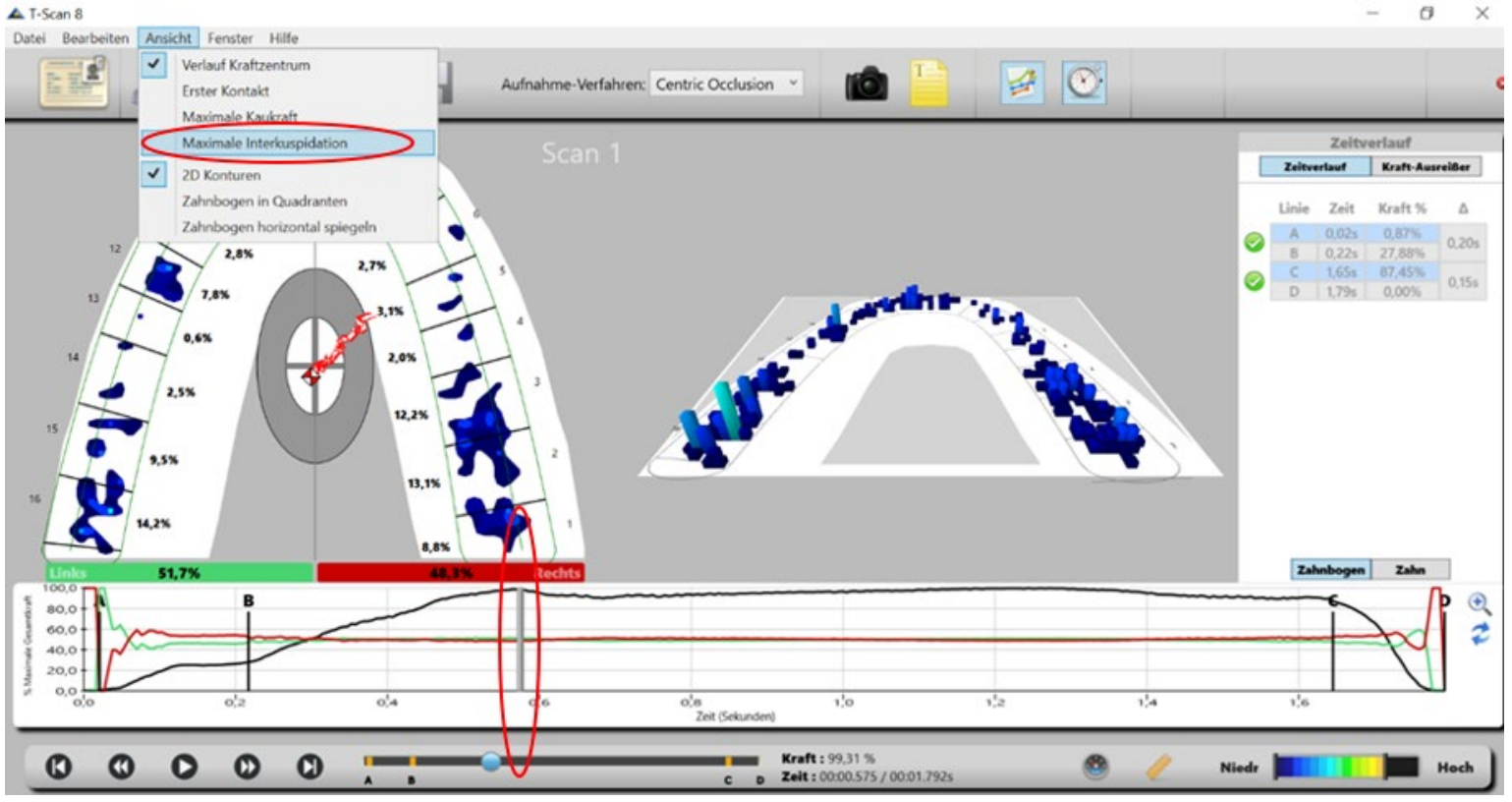

Abbildung 22: T-Scan-III-Analyseoberfläche der statischen Okklusion. Auswertungsansicht der statischen Okklusion zum Zeitpunkt der maximalen Interkuspidation (rot) eines/-er Probanden/-in in der Software T-Scan 8.1.

Um die dynamische Okklusion mittels T-Scan-III-System analysieren zu können, war die Festlegung eines weiteren Zeitpunktes vorzunehmen. Hierbei musste der Zeitpunkt der Exkursionsbewegung des Unterkiefers aufgesucht werden, an dem die prozentuale Beteiligung ipsilateraler Führungszähne beim erstmaligen Erreichen von 100 \% Gesamtkaukraft auf der Seite der durchgeführten Bewegungsrichtung festzustellen war (Abb. 23). 


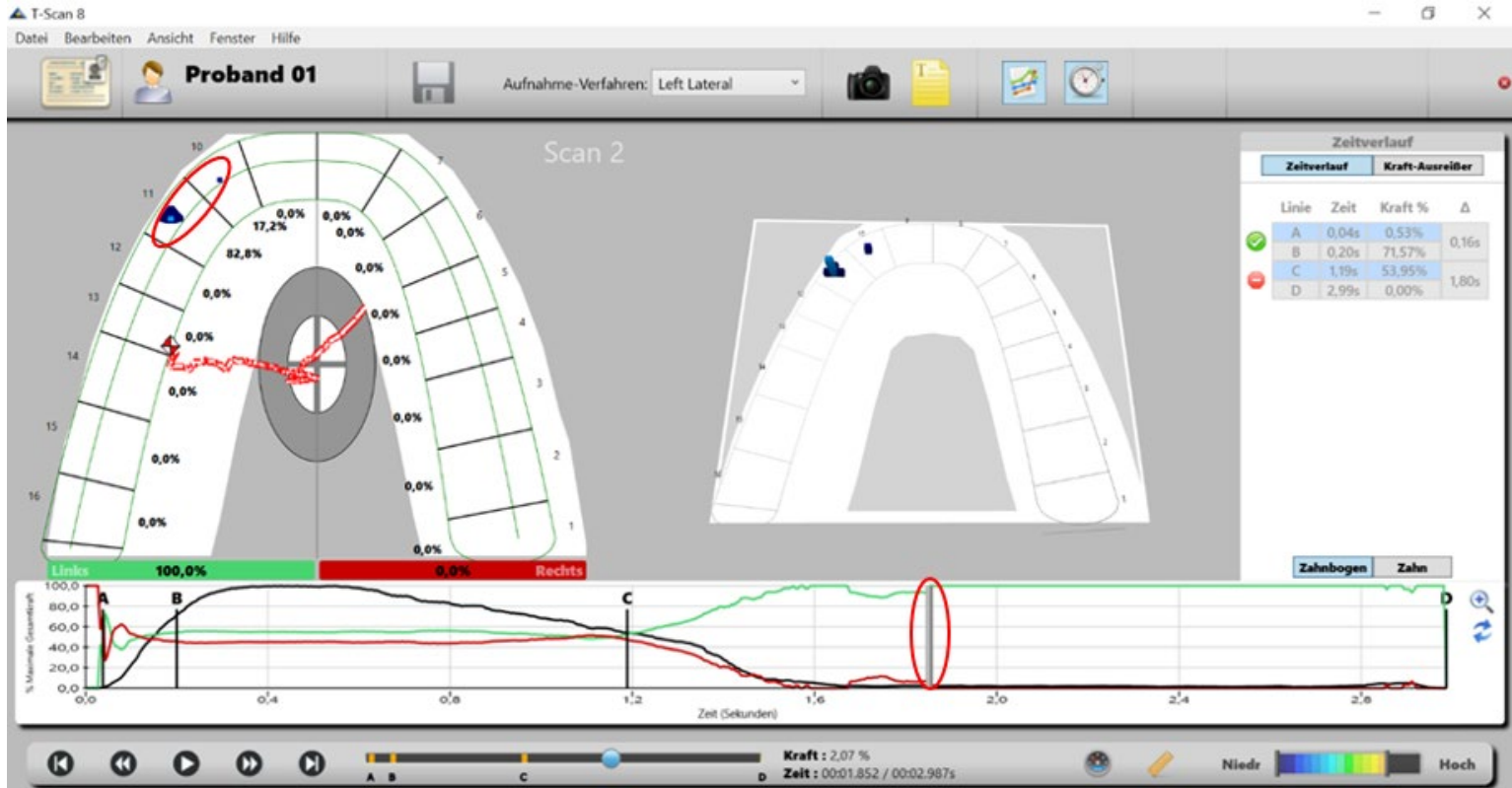

Abbildung 23: T-Scan-III-Analyseoberfläche der dynamischen Okklusion nach links. Auswertungsansicht der dynamischen Okklusion nach links eines/-er Probanden/-in bei erstmaligem Erreichen von 100\% Gesamtkaukraft der Arbeitsseite (rot), in der Software T-Scan 8.1 .

Konnte die Führungsfunktion kontralateraler Zähne nicht ausgeschlossen werden, wurde folgendermaßen vorgegangen:

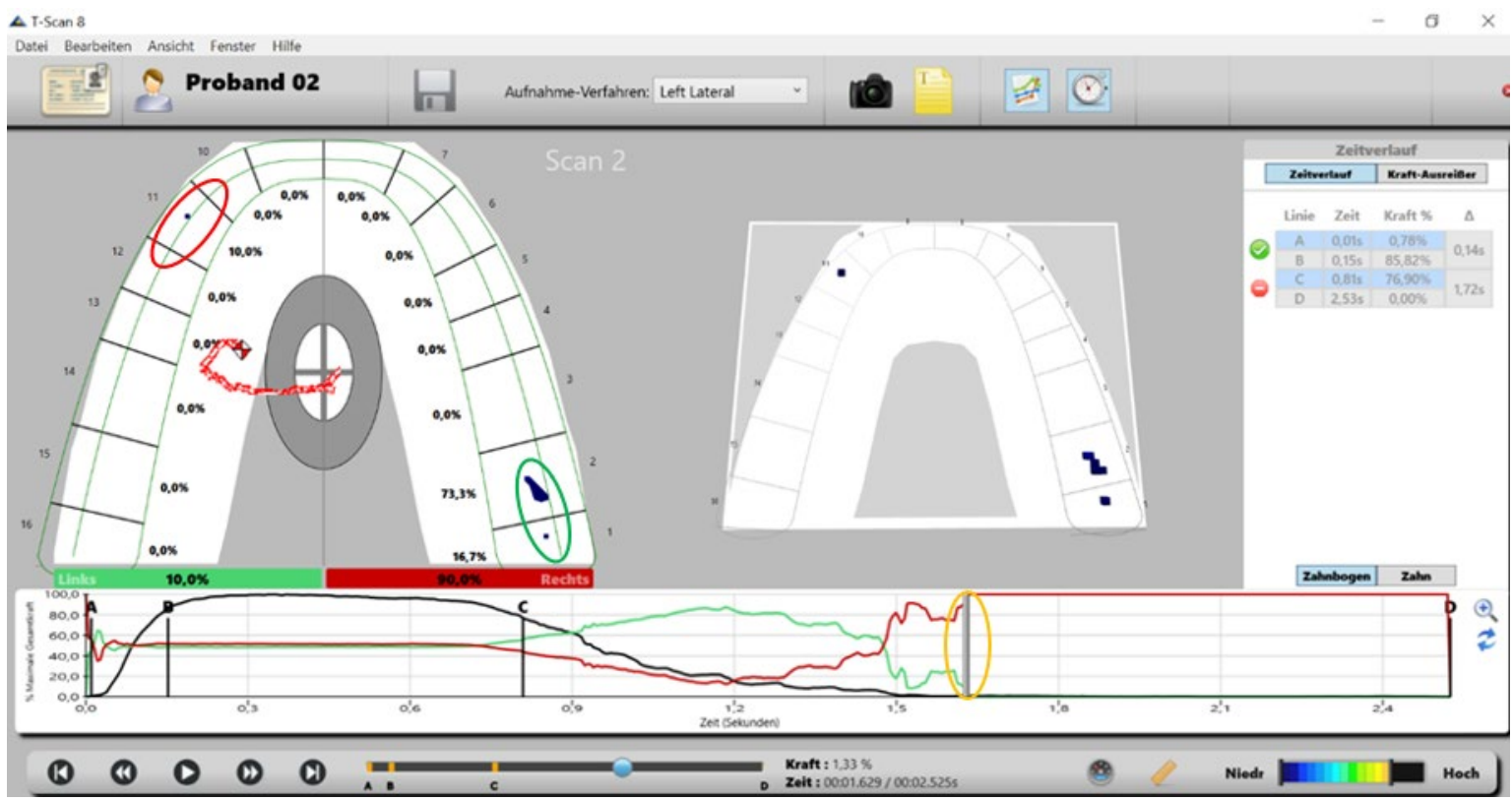

Abbildung 24: T-Scan-III-Analyseoberfläche der hyperbalancierten Okklusion. Auswertungsansicht der dynamischen Okklusion nach links bei erstmaligem Erreichen von $100 \%$ Gesamtkaukraft (orange) mit Führung des Eckzahnes (rot) der Arbeitsseite und der Molaren (grün) der Gegenseite eines/-er Probanden/-in in der Software T-Scan 8.1. 
In Abbildung 24 dargestelltes Registrat zeigt die zahngeführte Unterkieferbewegung nach links. Hierbei sollte normalerweise der linke, grüne Graph am Ende der Laterotrusion bei 100 \% liegen.

In aufgeführtem Beispiel wird die vornehmliche Führungsrolle aber durch die Zähne der Balanceseite übernommen (Abb. 24 grün), da trotz linksgerichteter Bewegung der rechte, rote Graph gegen $100 \%$ strebt. Bei weiterer Verlaufsbetrachtung ist die finale Führungsrolle deutlich in der kontralateralen Molarenregion zu sehen (unilateral balancierte Okklusion durch die Nichtarbeitsseite) (Abb. 25 rot):

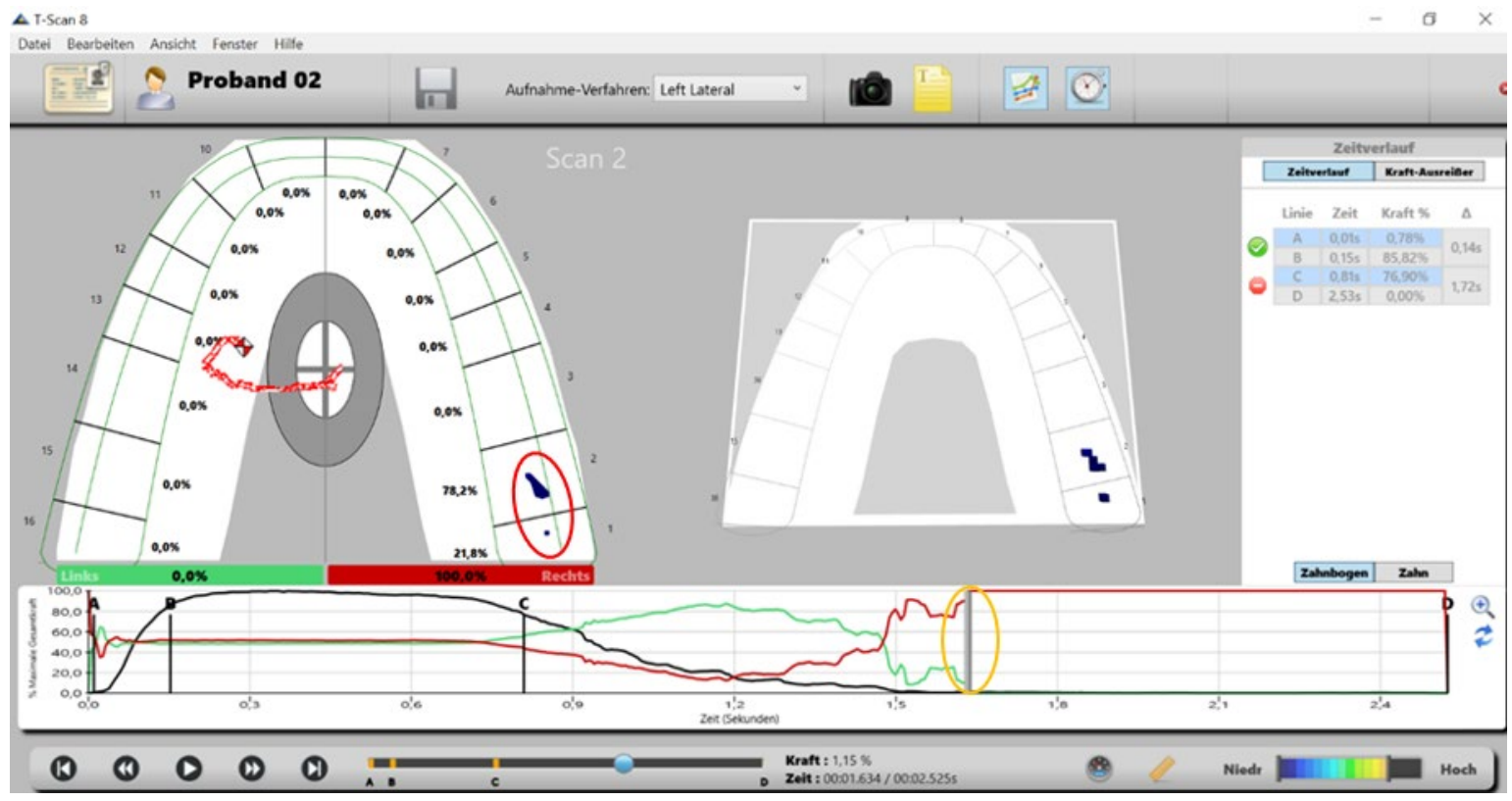

Abbildung 25: Justierung der T-Scan-III-Analyseoberfläche. Justierung der Auswertungsansicht der dynamischen Okklusion nach links mit Führung der Molaren der Gegenseite eines/-er Probanden/-in in der Software T-Scan 8.1 (Tekscan, South Boston, USA)

Lag ein derartiges Verzahnungsmuster vor, wurde der Graphenschlitten (Abb. 25 orange) derart platziert, dass der gerade noch registrierbare Führungszahn der eigentlichen Arbeitsseite kurz vor Erreichen der $100 \%$ der Gegenseite erfasst werden konnte. Die genaue Position ist auf Abbildung 25 erkennbar.

Im Anschluss erfolgte die Erfassung der prozentualen Beteiligung jedes Oberkieferzahnes an der gesamt applizierten relativen Kaukraft zum definierten Zeitpunkt der maximalen Interkuspidation, sowie die prozentuale Kraftverteilung der rechten und linken Seite des Zahnbogens zur Analyse der statischen Okklusion. Die dynamische Okklusion wurde unter Berücksichtigung der vorgestellten Kriterien erfasst. Sämtliche erhobenen Werte wurden anschließend auf dem Tagesprotokoll des/der jeweiligen Probanden/-in festgehalten. 


\subsubsection{Auswertung der Silikonregistrate}

Die Auswertung der Silikon-Bissregistrate zur Erhebung der statischen Okklusion erfolgte mit einem Tasterzirkel Kroeplin E110W (Kroeplin GmbH, Schlüchtern, Deutschland) (Abb. 26). Hierzu wurden sämtliche durchscheinende Areale des Bissregistrates auf ihre Materialstärke überprüft. Ein Kontaktpunkt lag gemäß des verwendeten Studienprotokolls immer dann vor, wenn die zu untersuchende Kontaktpunktstelle eine Stärke von $\leq 20 \mu \mathrm{m}$ aufwies. Durchscheinende Areale mit einer Schichtstärke von $\geq 20 \mu \mathrm{m}$ wurden nicht aufgenommen. Das hohe Auflösungsvermögen des Registrates ermöglichte eine klare Zuordnung der Kontaktareale des betroffenen Zahnes. Ein Kontaktpunkt lag immer dann vor, wenn eine homogene, runde oder ovale Fläche ohne Septierung (Abb. 27), also solider Abgrenzung zu sehen war. Vorliegende Septierungen (Abb. 27) unterteilten ein Kontaktflächenareal in mehrere Kontaktpunkte, sofern die Schichtstärke von $20 \mu \mathrm{m}$ nicht überschritten wurde. Sämtliche Kontaktpunkte wurden je Untersuchung auf dem Tagesprotokoll fixiert und nach Komplettierung der statistischen Analyse zugeführt.

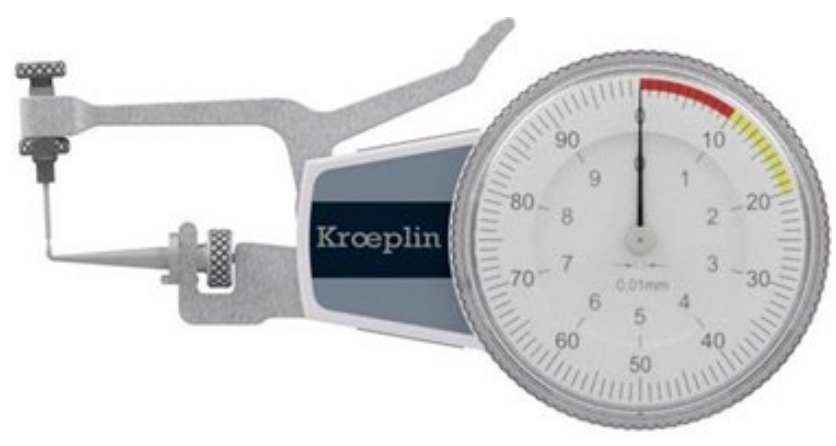

Abbildung 26: Tasterzirkel. E110W (Kroeplin GmbH, Schlüchtern, Deutschland). Die Verwendung erfolgt mit freundlicher Genehmigung der Firma Kroeplin. 


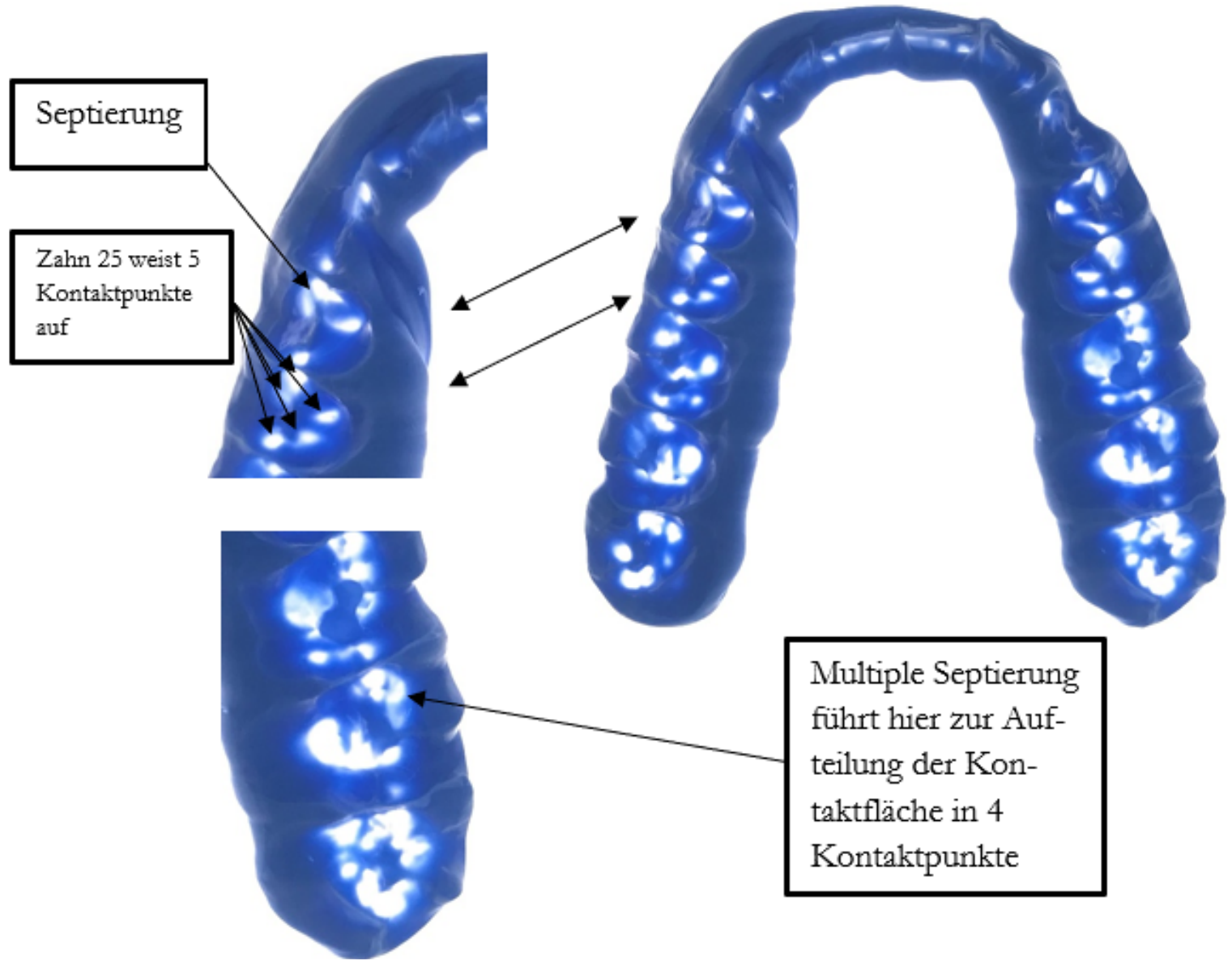

Abbildung 27: Auswertung der Silikonregistrierung.

\subsubsection{Auswertung der Wachsregistrate}

Die Auswertung der Wachsregistrate zur Erhebung der statischen Okklusion wurde ebenfalls mit dem Tasterzirkel Kroeplin E110W vorgenommen (Abb. 26). Sämtliche durchscheinende Areale des Bissregistrates wurden auf ihre Materialstärke hin überprüft. Ein Kontaktpunkt lag in dem verwendeten Studienprotokoll immer dann vor, wenn die zu untersuchende Kontaktpunktstelle eine Stärke von $\leq 20 \mu \mathrm{m}$ aufwies. Durchscheinende Areale mit einer Schichtstärke $\geq 20 \mu \mathrm{m}$ wurden nicht aufgenommen. Ein Kontaktpunkt lag immer dann vor, wenn eine homogene, runde oder ovale Fläche ohne Septierung (Abb. 28), also ohne solide Abgrenzung, zu sehen war. Vorliegende Septierungen (Abb. 28) unterteilten ein Kontaktflächenareal in mehrere Kontaktpunkte, sofern die Schichtstärke von $20 \mu \mathrm{m}$ nicht überschritten wurde. Sämtliche Kontaktpunkte wurden je Untersuchung auf dem Tagesprotokoll fixiert und nach Komplettierung der statistischen Analyse zugeführt. 


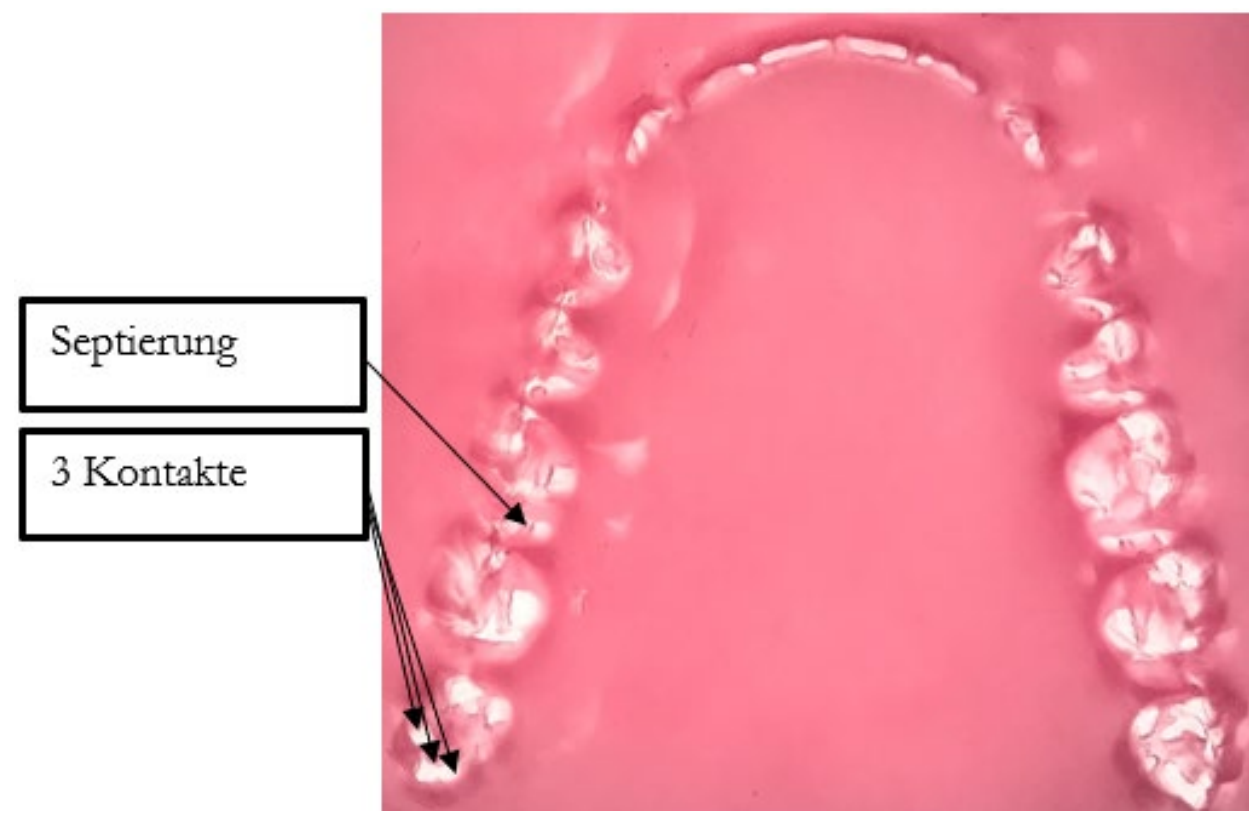

Abbildung 28: Auswertung der Wachsregistrierung.

\subsubsection{Auswertung der Kontaktfolienmessung}

Nach Durchführung des bereits beschriebenen Shimstock-Okklusionsprotokolls wurden auf Zug registrierte Kontakte, die zu einer Verklemmung zwischen den Zahnreihen führten (Abb. 18 a), als „positiv“ (+) deklariert (Abb. 18 b). War die Folie reibungslos aus dem Okklusionsspalt zu führen, wurden keine Kontakte registriert und der Zahnantagonismus folglich als „negativ“ $(-)$ notiert. Konnte die Folie unter deutlichem Widerstand aus dem Zahnantagonismus entfernt werden, lag ein regelrechter Kontaktpunkt vor. Dieser wurde zur besseren Differenzierung als „positiv/negativ“ (+/-) bezeichnet. Sämtliche Befunde wurden tabellarisch notiert (Abb. 18 b).

\subsubsection{Auswertung der Farbfolienmessung}

Die zuvor erhobenen Okklusionsmuster wurden fotografiert und katalogisiert. Zur Beurteilung der statischen und dynamischen Okklusion wurden die verschiedenfarbigen Kontaktareale zuerst der jeweiligen Okklusion zugeordnet. Die schwarz färbende Folie registrierte hierbei die statische Okklusion in habitueller Interkuspidation (Abb. 29). Die rot färbende Folie markierte die dynamische Okklusion (Abb. 30). In der Betrachtung wurden neben eindeutigen Kontaktpunkten auch Linien berücksichtigt, die üblicherweise in der dynamischen Okklusion feststellbar waren (Abb. 30). 


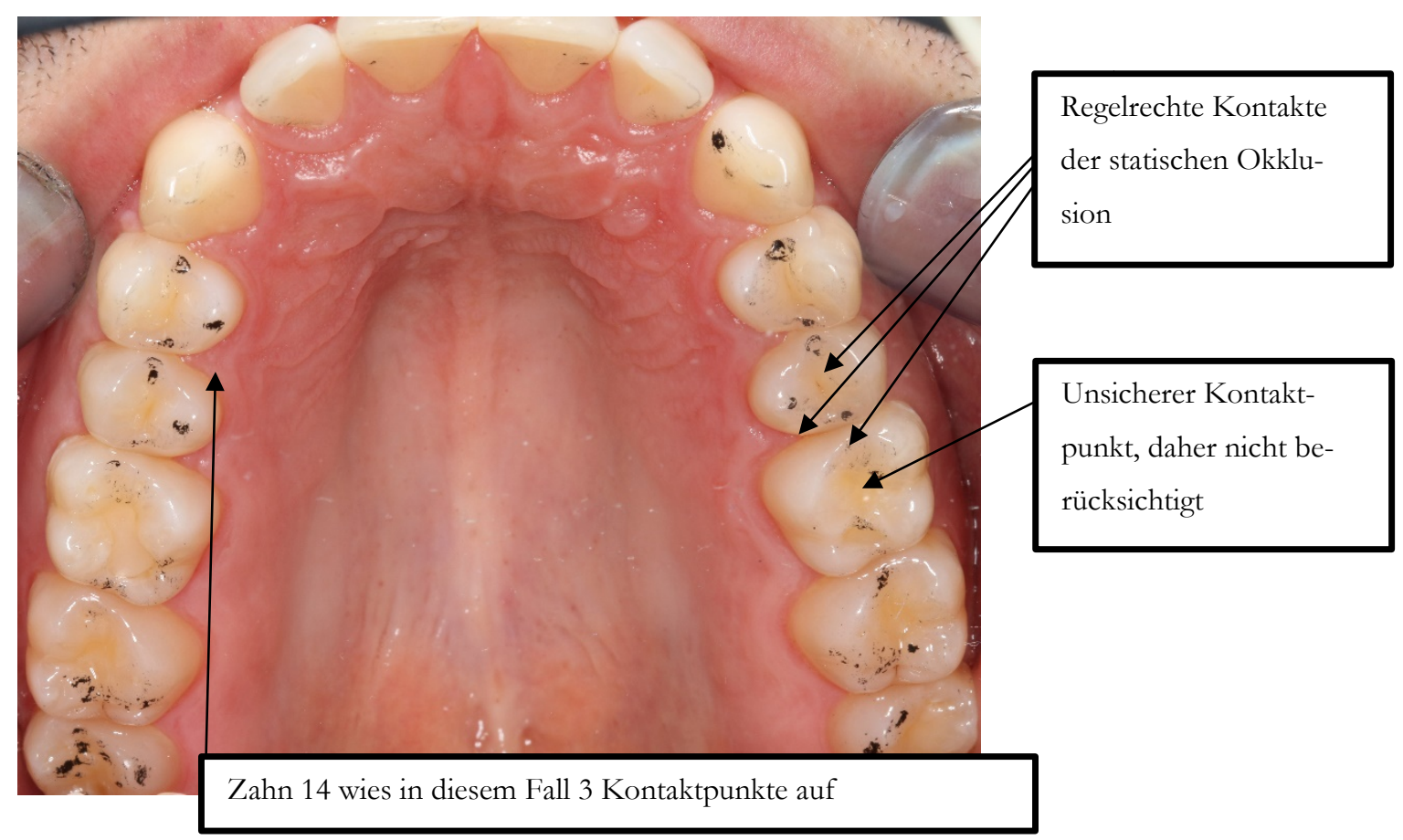

Abbildung 29: Okklusionsfolienauswertung (statisch). Auswertung der statischen Okklusion mittels Okklusionsfolie schwarz $12 \mu \mathrm{m}$.

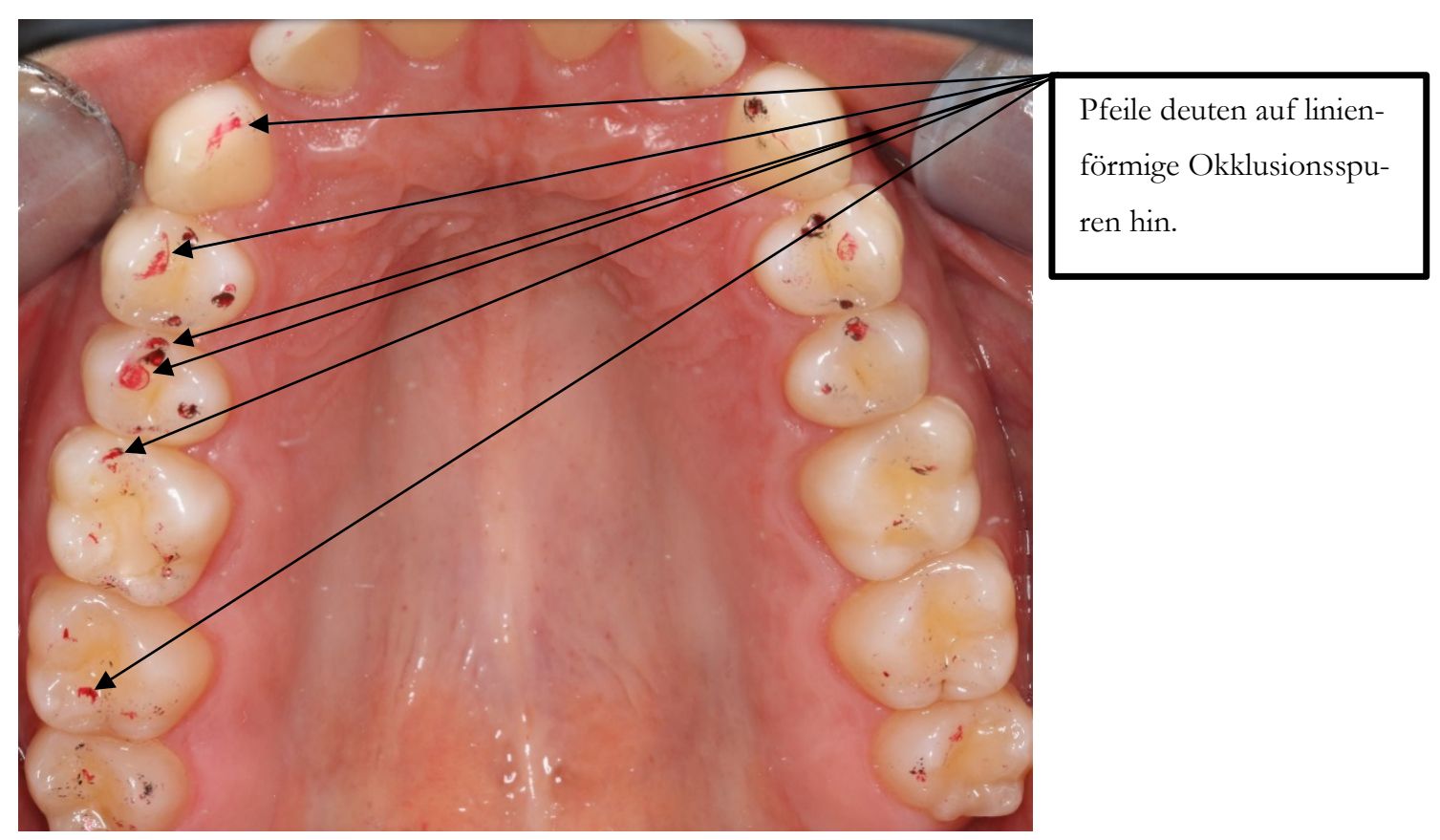

Abbildung 30: Okklusionsfolienauswertung (dynamisch). Auswertung der dynamischen Okklusion nach rechts mittels Okklusionsfolie rot $12 \mu \mathrm{m}$.

Abbildung 30 zeigt die Erfassung der dynamischen Okklusion nach rechts. Hierbei sind ausschließlich die Markierungen im Sinne einer Kontaktlinie zu registrieren, die aus der habituellen Interkuspidationsposition entspringen und nachverfolgbar linien- oder bogenförmig abgehen. 
Das dargestellte Beispiel zeigt an sechs Markierungen die zahngeführte Unterkieferexkursion nach rechts. Führungskontakte der kontralateralen Seite im Sinne einer Hyperbalance waren nicht feststellbar.

Die Übertragung der registrierten Kontaktpunkte in statischer und dynamischer Okklusion komplettierte das Tagesprotokoll. Die Gesamtheit aller Daten wurde in eine zusammenhängende Excel-Tabelle aller Probanden/-innen eingepflegt.

\subsubsection{Statistische Methoden}

Die statistische Auswertung wurde von der zentralen Serviceeinheit Medizinische Biometrie und statistische Bioinformatik des Instituts für medizinische Statistik der Universitätsmedizin Göttingen unterstützt. Die Darstellung der Daten erfolgte zusammenfassend mittels absoluter und relativer Häufigkeiten für die kategoriellen Variablen. Die Darstellung der kontinuierlichen Variablen erfolgte anhand des Mittelwertes und der jeweiligen Standardabweichung (standard deviation, SD) (Mittelwert $\pm \mathrm{SD}$ ), sowie des Medians aus minimaler und maximaler Größe (Median, min; max). Ordinal skalierte Variablen wurden ebenfalls mittels Median aus minimaler und maximaler Größe (Median, min; max) dargestellt.

Die Messungen der verschiedenen Okklusions-Registrierverfahren wurden paarweise korreliert. Hierzu fanden der Pearsons Korrelationskoeffizient sowie der Kendalls Rangkorrelationskoeffizient (Kendalls $\tau$ ) Anwendung. Die resultierenden p-Werte wurden mittels Bonferroni-HolmKorrektur für multiples Testen adjustiert.

Für die Modellierung wurden die erhobenen Daten kategorisiert. Ausgehend von der Okklusion zum ersten Zeitpunkt wurde pro Zahn zum nächsten Zeitpunkt eine Erhöhung der Okklusion kodiert, wenn sich die Kontaktpunktanzahl oder die relative prozentuale Beteiligung eines Zahnes an der gesamt applizierten Kaukraft um $\geq 5 \%$ erhöhte. Eine Verringerung der Okklusion wurde bei einer Reduktion der Kontaktpunktanzahl respektive der prozentualen Beteiligung eines Zahnes um $\geq 5 \%$ gesehen. Die unveränderte Okklusion wurde bei gleichbleibender Kontaktpunktanzahl bzw. bei Veränderung der relativen prozentualen Beteiligung eines Zahnes um $<5 \%$ kodiert. Potenzielle Einflussfaktoren auf die Wahrscheinlichkeit, Veränderungen in der Okklusion zu erkennen, wurden anhand der gemischten logistischen Regression unter Berücksichtigung der Messwiederholungen pro Probanden/-in untersucht. Für die Gesamtheit aller statistischen Tests wurde das Signifikanzniveau auf $\alpha=5 \%$ gesetzt. Sämtliche Analysen wurden in der statistischen Programmierumgebung R (Version 3.5.0; R Core Team 2018) durchgeführt. Hierbei fand das Paket Lme4 für die gemischte logistische Regression besondere Anwendung (Bates et al. 2015). 


\section{Ergebnisse}

Zur Vermeidung einer Pseudogenauigkeit erfolgte die Rundung der erhobenen Werte auf eine Dezimalstelle. Im Rahmen der statistischen Auswertung wurden die erhobenen Werte auf bis zu drei Dezimalstellen nach dem Komma angegeben.

Die statistische Auswertung (detailliert dargestellt in den folgenden Kapiteln) lieferte zusammengefasst folgende klinische Erkenntnisse:

Die Verfahren HS-Modellierwachs und Registrado zeigten den größten korrelativen Zusammenhang bei Okklusionsänderungen im Sinne einer $\mathrm{Zu}$ - oder Abnahme von Kontaktpunkten (Abb. 31 und 32). Konnte das Verfahren Registrado eine Okklusionsänderung um $\geq 5 \%$ nachweisen, war dies mit hoher Wahrscheinlichkeit auch bei HS-Modellierwachs der Fall. Kam es in einem Verfahren zu einer Zunahme der Okklusionskontakte, stellte das andere Verfahren ebenfalls eine entsprechende Zunahme fest. Durch den positiv korrelierenden Zusammenhang ergab sich das gleiche Verhalten bei Okklusionsabnahmen. Der zugehörige signifikante p-Wert von $<0,001$ zeigt, dass der beobachtete Zusammenhang nicht zufällig war.

Ob die Ursache für diese Änderungen rein Methodenbedingt war oder durch tageszeitliche Schwankungen der Okklusion ausgelöst wurde, sollte ebenfalls untersucht werden. Geringere aber immer noch starke Korrelationen zu der Wachs- und Silikonregistrierung zeigten die Verfahren Okklusionsfolie $12 \mu \mathrm{m}$ (statisch) und T-Scan III (statisch). Das Shimstock-Verfahren zeigte die geringste Korrelation zu den anderen Methoden der statischen Okklusionsregistrierung (Abb. 31 und 32). Hinsichtlich des Vergleichs der Methoden zur Erhebung der statischen und dynamischen Okklusion, zeigten das Verfahren T-Scan III (dynamische Okklusion nach rechts und links) erwartungsgemäß keine signifikante Korrelation mit den Verfahren zur Erhebung der statischen Okklusion (Abb. 31 und 32). Das Verfahren Okklusionsfolie $12 \mu \mathrm{m}$ zur Erhebung der dynamischen Okklusion nach rechts und links korrelierte positiv und signifikant mit allen Verfahren der statischen Okklusion. Hier konnte bei einer exemplarischen Zunahme von Kontaktpunkten in der statischen Okklusion auch eine Zunahme der Kontakte bei dynamischer Okklusion festgestellt werden. Zusätzlich konnte eine positive signifikante Korrelation, der Verfahren zur Erhebung der dynamischen Okklusion untereinander festgestellt werden. Konnten exemplarisch mittels Okklusionsfolie $12 \mu \mathrm{m}$ zur Erhebung der dynamischen Okklusion nach links mehr Kontaktpunkte nachgewiesen werden, so war dies in der Regel auch mittels T-Scan-III-Registrierung zur Erhebung der dynamischen Okklusion nach links der Fall. Der Vergleich der Verfahren T-Scan-III zur Erhebung der dynamischen Okklusion nach rechts und links konnte eine negative signifikante Korrelation zeigen. 
So konnte exemplarisch bei einer zahngeführten Unterkieferexkursion nach rechts eine Zunahme der Zahnkontakte auf der rechten Seite (Arbeitsseite) und gleichzeitig eine Abnahme der Zahnkontakte auf der linken Seite (Nichtarbeitsseite) festgestellt werden (Abb. 31 und 32). Anders verhielt es sich im Vergleich der Verfahren zur Erhebung der dynamischen Okklusion nach rechts und links mittels Okklusionsfolie $12 \mu \mathrm{m}$. Hier konnte eine positive, signifikante Korrelation der Verfahren festgestellt werden (Abb. 31 und 32). So führte eine Kontaktpunktzunahme der Arbeitsseite ebenfalls zu einer Kontaktpunktzunahme der Nichtarbeitsseite.

Hinsichtlich der erkannten Änderungen der Kontaktpunkte je Zahn, wurde mit dem Verfahren Okklusionsfolie $12 \mu \mathrm{m}$ der größte Änderungsgrad der Okklusion festgestellt. Darauf folgten absteigend die Verfahren T-Scan-III zur Erhebung der dynamischen Okklusion, Registrado Xtra, Okklusionsfolie $12 \mu \mathrm{m}$ zur Erhebung der dynamischen Okklusion, T-Scan-III zur Erhebung der statischen Okklusion sowie HS-Modellierwachs. Mit der Shimstock-Registrierung wurde der geringste Änderungsgrad der Okklusion gemessen. Alle Verfahren, außer Shimstock, zeigten den größten Änderungsgrad der Okklusion im Seitenzahnsegment an, wobei die ersten und zweiten Molaren am stärksten betroffen waren. Das Ausmaß der Änderung nahm, außer in der Shimstock-Registrierung, zum Frontzahnsegment hin ab, wobei die HSModellierwachsregistrierung den geringsten Änderungsgrad im Frontzahnsegment zeigte und die Shimstock-Registrierung die Höchste. Die Verfahren zur Erhebung der dynamischen Okklusion zeigten den höchsten Änderungsgrad, anders als die der statischen Okklusion, an den Eckzähnen. Für jeden Zahn wurde immer dann eine Okklusionsänderung gesehen, wenn die Anzahl der Kontaktpunkte verändert war, bzw. sich die relative Kaukraftverteilung um mehr als $5 \%$ geändert hat.

Die Wahrscheinlichkeit, gegenüber der Messung um 9 Uhr eine Okklusionsänderung an einem Zahn festzustellen, war über alle Zähne hinweg in jedem Verfahren signifikant geringer als $50 \%$. Allerdings unterschieden sich hier die Registrate deutlich. Die Registrierung der statischen Okklusion mittels Okklusionsfolie $12 \mu \mathrm{m}$ zeigte mit einer Wahrscheinlichkeit von 41,6\% am ehesten eine Okklusionsänderung an einem beliebigen Zahn. Die Registrado-Registrierung konnte mit 18,4 \%-iger Wahrscheinlichkeit eine Änderung feststellen. Die Erhebung der dynamischen Okklusion mittels T-Scan III wies mit $15 \%$-iger-, die Okklusionsfolie $12 \mu \mathrm{m}$ (dynamische Okklusion) mit $8 \%$-iger und die Registrierung der statischen Okklusion mittels T-Scan III mit 7,5\%-iger Wahrscheinlichkeit eine Änderung nach. Die Shimstock- und HSModellierwachsregistrierung zeigten mit 3\%-iger Wahrscheinlichkeit eine Änderung an. Auch die Zähne unterschieden sich hinsichtlich der Wahrscheinlichkeit für eine Okklusionsänderung. Bei den Inzisiven und Caninen konnte eine 5-6\%-ige Wahrscheinlichkeit festgestellt werden. 
Die Prämolaren wiesen mit einer 12\%-igen und die Molaren mit einer 15-18\%-igen Wahrscheinlichkeit eine Änderung in einer Okklusionsmessung auf.

Eine Okklusionsänderung pro Kiefer wurde in dieser Studie immer dann gewertet, wenn mindestens zwei Zähne je Messung eine Okklusionsänderung um $\geq 5 \%$ zeigten. Die Wahrscheinlichkeit eine Okklusionsänderung im Kiefer festzustellen, war in allen Verfahren deutlich höher als bei der Bewertung eines einzelnen Zahnes. So bestand bei der Registrierung der statischen Okklusion mittels Okklusionsfolie $12 \mu \mathrm{m}$ eine 98,8\%-ige Wahrscheinlichkeit, in einer Okklusionsmessung eine Änderung festzustellen. Die Registrado-Registrierung zeigte mit 66,3\%-iger Wahrscheinlichkeit eine Änderung in der Okklusionsmessung an. Die übrigen Verfahren zur Erhebung der statischen Okklusion: T-Scan, Shimstock und Wachs zeigten mit 20\%-iger, 14\%iger bzw. 7\%-iger Wahrscheinlichkeit eine Änderung der Okklusion im Kiefer an. Die Verfahren zur Erhebung der dynamischen Okklusion zeigten mit einer Wahrscheinlichkeit von $70 \%$ im Falle des T-Scans und $30 \%$ mittels Okklusionsfolien-Registrierung eine Änderung der Okklusion an. Es konnte also gezeigt werden, dass die Wahrscheinlichkeit eine Okklusionsänderung zu registrieren in allen Verfahren deutlich anstieg, sobald bei Auswertung aller Zähne mindestens zwei Zähne eine Änderung aufwiesen. Die Untersuchungszeitträume von 9- zu 12 Uhr und 9- zu 16 Uhr hatten in keinem Verfahren einen signifikanten Unterschied in der Chance, eine Änderung der Okklusion festzustellen. Es ist daher anzunehmen, dass sich die Okklusion zu jeder Tageszeit anders darstellt (also nicht konstant ist), jedoch die drei festgelegten Messzeitpunkte nicht mit höherer Chance eine Änderung der Okklusion zeigen.

\section{1 Übersicht der Untersuchung}

Tabelle 3: Übersicht der durchgeführten Messungen

\begin{tabular}{|c|c|}
\hline Zahn & $\begin{array}{c}\text { Anzahl der Messungen insgesamt bei al- } \\
\text { len Teilnehmenden in } \mathbf{n} \text { (\%) }\end{array}$ \\
\hline 11 & $114(7,1)$ \\
\hline 12 & $114(7,1)$ \\
\hline 13 & $114(7,1)$ \\
\hline 14 & $114(7,1)$ \\
\hline 15 & $102(6,3)$ \\
\hline 16 & $114(7,1)$ \\
\hline
\end{tabular}




\begin{tabular}{|c|c|}
\hline Zahn & $\begin{array}{c}\text { Anzahl der Messungen insgesamt bei al- } \\
\text { len Teilnehmenden in } n(\%)\end{array}$ \\
\hline 17 & $114(7,1)$ \\
\hline 18 & $18(1,1)$ \\
\hline 21 & $114(7,1)$ \\
\hline 22 & $114(7,1)$ \\
\hline 23 & $114(7,1)$ \\
\hline 24 & $114(7,1)$ \\
\hline 25 & $102(6,3)$ \\
\hline 26 & $114(7,1)$ \\
\hline 27 & $114(7,1)$ \\
\hline 28 & $18(1,1)$ \\
\hline $\begin{array}{c}\text { Messungen je Registrierverfahren bei } 19 \\
\text { Probanden/-innen insgesamt }\end{array}$ & 1608 \\
\hline $\begin{array}{c}\text { Registrierungen aller Verfahren und aller } \\
\text { Zähne }\end{array}$ & 14472 \\
\hline
\end{tabular}

Die angeführte Tabelle 3 zeigt die Gesamtheit der untersuchten Zähne von 19 Probanden/innen an zwei Untersuchungstagen zu jeweils drei Messungen je Verfahren. Die Abweichungen der Zähne 15 und 25 resultieren aus der Nichtanlage dieser Zähne bei zwei Probandinnen. Die Abweichungen der Zähne 18 und 28 resultieren aus der Nichtanlage dieser Zähne bei 16 Probanden/-innen.

Tabelle 4: Messwertverteilung je Quadrant

\begin{tabular}{|c|c|}
\hline Probanden/-innenseite & $\begin{array}{c}\text { Anzahl der Zähne nach sechsmaliger } \\
\text { Messung des gesamten Probandengutes } \\
\text { in n (\%) nach Seite }\end{array}$ \\
\hline links & $804(50,0 \%)$ \\
\hline rechts & $804(50,0 \%)$ \\
\hline
\end{tabular}


Tabelle 4 zeigt, dass sowohl auf der linken als auch rechten Kieferhälfte der Probanden/-innen gleichermaßen viele Registrierungen vorgenommen wurden.

\subsection{Korrelation der Verfahren}

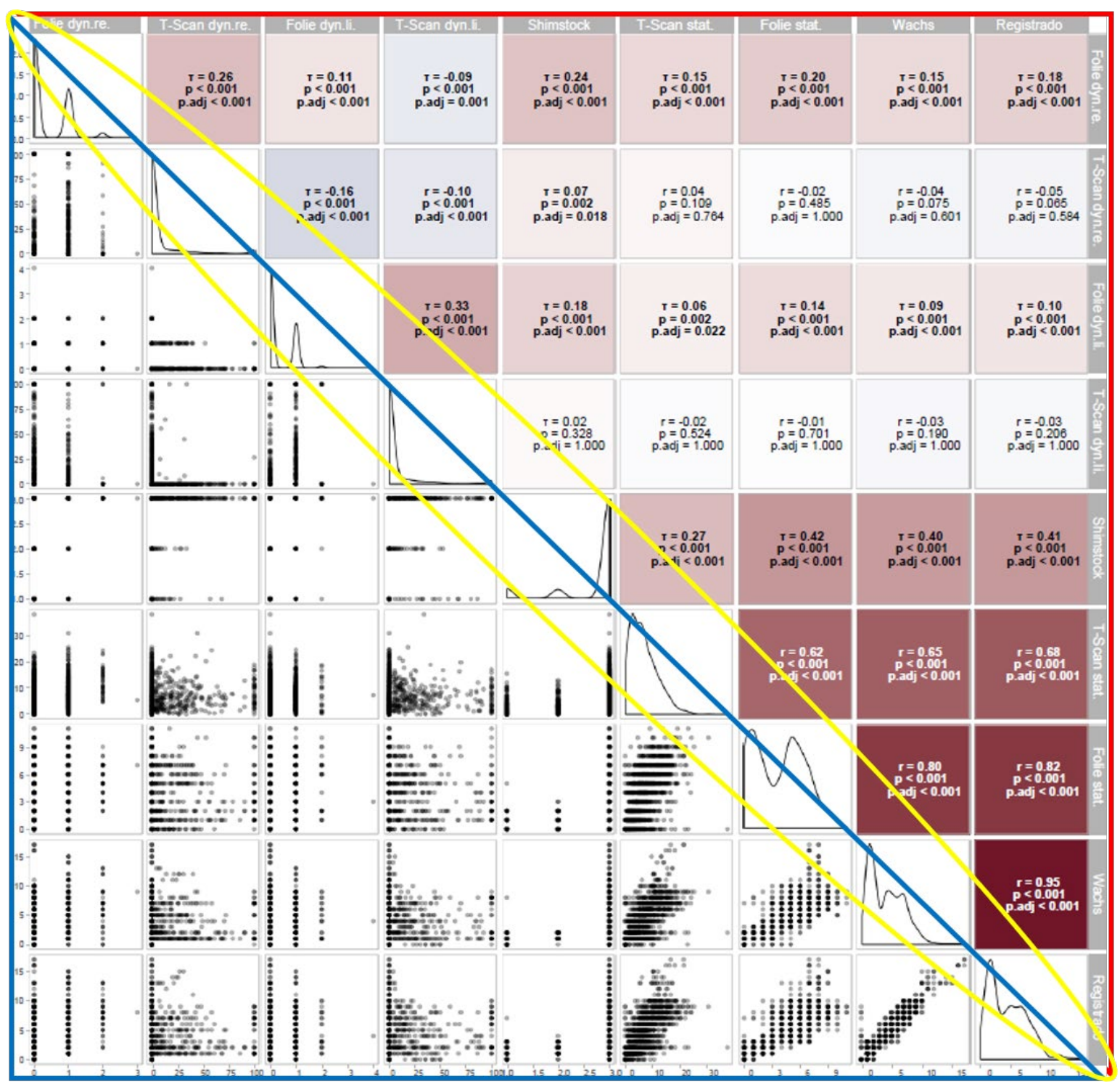

Abbildung 31: Paarweise Korrelation der verschiedenen Okklusionsregistrierungen.

Die Grafik in Abbildung 31 stellt die paarweise Korrelation der durchgeführten Registrierverfahren dar. Hierbei zeigt die untere Dreiecksmatrix (blau) die paarweisen Scatterplots (Punktewolken), also die Beziehung der einen zur anderen Methode. Die Diagonalfelder (gelb) zeigen mit Hilfe der empirischen Dichte die Verteilung der Messungen. Die obere Dreiecksmatrix (rot) führt die Korrelationskoeffizienten, die p-Werte für die Testung gegen 0 sowie die nach Holm adjustierten p-Werte auf. Die blau hinterlegten Felder zeigen eine negative Korrelation der paarweise untersuchten Methoden. 
Rot hinterlegte Tafeln geben eine positive- und fett gedruckte Werte eine signifikante Korrelation an. Die obere rote Dreiecksmatrix drückt also diagonal gespiegelt die korrespondierenden Punktewolken zweier korrelierender oder nicht korrelierender Methoden (blaue Dreiecksmatrix) aus. Starke Methodenkorrelationen werden in dunkel-, schwache in hellroten Tafeln dargestellt. Somit ist augenscheinlich, dass die stärkste Korrelation der durchgeführten Methoden zwischen HS-Modellierwachs und Registrado X-tra bestand. Die Korrelation drückt aus, dass eine Kontaktpunktzu- oder -abnahme sowohl mit HS-Modellierwachs als auch mit Registrado X-tra in enger Übereinstimmung $(r=0,95)$ festgestellt werden konnte. Ein etwas schwächerer, aber immer noch stark korrelierender Zusammenhang konnte mit der Okklusionsfolie $12 \mu \mathrm{m}$ zur Erhebung der statischen Okklusion gesehen werden $(r=0,82)$. Der schwächste Zusammenhang der stark korrelierenden Methoden wurde zwischen der Erhebung der statischen Okklusion mittels T-Scan III zu Registrado X-tra, HS-Modellierwachs und Okklusionsfolie $12 \mu \mathrm{m}$ gesehen. Der Korrelationskoeffizient lag hier bei 0,62 für die Übereinstimmung mit Okklusionsfolie $12 \mu \mathrm{m}$, bei 0,65 für jene des HS-Modellierwachses und bei 0,68 für die Bissnahme mit Registrado X-tra.

Unter Berücksichtigung der dargestellten Zusammenhänge fiel auf, dass jede der dargestellten Methoden immer dann den höchsten Korrelationskoeffizienten erreichte, wenn sie mit Registrado X-tra korreliert wurde.

Für die Korrelation der Untersuchungsmethoden mit Shimstock-Folie wurde der Rangkorrelationskoeffizient Kendalls Tau ermittelt. Bei den Methoden zur Erhebung der statischen Okklusion konnte eine Korrelation der Shimstock-Folie mit dem T-Scan III, der Okklusionsfolie $12 \mu \mathrm{m}$, dem HS-Modellierwachs und dem Registrado X-tra festgestellt werden. Hinsichtlich der Erhebung der dynamischen Okklusion wurde ebenfalls die Korrelation der Methoden durch Kendalls Tau ermittelt. Die Erhebung der dynamischen Okklusion nach links mittels Okklusionsfolie $12 \mu \mathrm{m}$ zeigte eine positive Korrelation $(\tau=0,33)$ mit der Erfassung der Laterotrusion nach links mittels T-Scan III. Zu- und Abnahme der Kontakte konnten also mit beiden Methoden in signifikantem Zusammenhang ermittelt werden. Bei der Laterotrusion nach rechts konnte ebenfalls eine positive Korrelation $(\tau=0,26)$ zwischen T-Scan III und Okklusionsfolie $8 \mu \mathrm{m}$ festgestellt werden. Betrachtet man die paarweise Korrelation der Methode T-Scan III zur Erfassung der Laterotrusion nach rechts mit der Erhebung der dynamischen Okklusion nach links anhand der Okklusionsfolie $12 \mu \mathrm{m}$, so kann eine negative Korrelation $(\tau=-0,10)$ festgestellt werden. So führte in der Mehrheit der Untersuchungen eine Kontaktpunktzunahme der Arbeitsseite zu einer Kontaktpunktabnahme der Gegenseite. 
Der gleiche Zusammenhang war bei der Erhebung der dynamischen Okklusion nach links mittels T-Scan III und der Laterotrusion nach rechts anhand der Okklusionsfolie $12 \mu \mathrm{m}$ zu sehen $(\tau=-0,09)$. Die negative Korrelation der Methode T-Scan III dynamisch links zu rechts unterstützt diese Feststellung. So sind die erhobenen Werte der T-Scan-III-Registrierung der linken Seite negativ zu denen der rechten Seite korreliert $(\tau=-0,16)$. Dies bedeutet im Zusammenhang, dass aus der Kontaktpunktzunahme der Arbeitsseite eine Kontaktpunktabnahme der Nichtarbeitsseite resultiert. Die physiologische Disklusion der Nichtarbeitsseite konnte hingegen mittels Okklusionsfolie $12 \mu \mathrm{m}$ nicht nachgewiesen werden. Bei Registrierung der Unterkieferexkursion mittels Okklusionsfolie $12 \mu \mathrm{m}$ nach links bestand sogar eine positive Korrelation zur rechten Seite $(\tau=0,11)$. Demnach hat die Kontaktpunktzunahme der Arbeitsseite in der Mehrheit der Untersuchungen zur Kontaktpunktzunahme der Nichtarbeitsseite geführt.

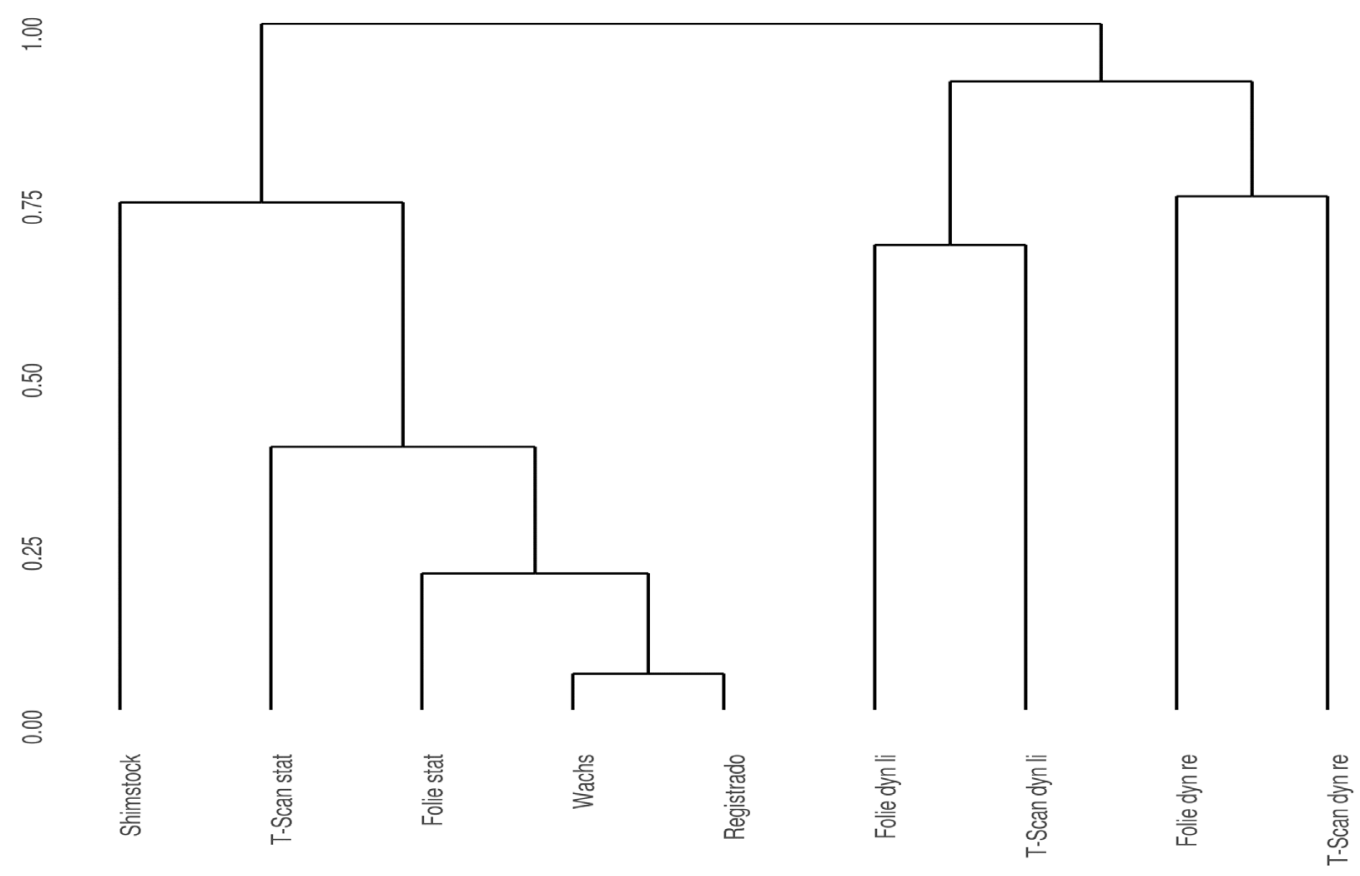

\section{Abbildung 32: Hierarchisches Clustering der Okklusionsregistrierungen nach Korrelation.}

Die Abbildung 32 zeigt das hierarchische Clustering der Okklusionsmessinstrumente. Die Verfahren wurden nach Korrelation hierarchisch gruppiert und in einem Dendrogramm dargestellt. Je kürzer die Äste des Baumes sind, desto höher ist die Korrelation zwischen den durch diese Äste verbundenen Verfahren. Aus Abb. 32 wird deutlich, dass vor allem die Verfahren Registrado X-tra, HS-Modellierwachs, Okklusionsfolie statisch und das T-Scan-IIISystem zur Ermittlung der statischen Okklusion stark korrelieren. 
Die besonders starke Korrelation zwischen Registrado X-tra und HS-Modellierwachs ist hierbei hervorzuheben. Hinsichtlich der Registrierung der dynamischen Okklusion mittels Okklusionsfolie und T-Scan-III-System wurde ein Clustering zur jeweiligen Seite der Bewegung ersichtlich. Aus den relativ hoch erscheinenden Clustern von Okklusionsfolie rechts, T-Scan III rechts sowie Okklusionsfolie links und T-Scan III links lässt sich im Vergleich zu den Verfahren zur Erfassung der statischen Okklusion eine geringere Korrelation ableiten. Im Vergleich der Verfahren T-Scan III rechts zu links und Okklusionsfolie rechts zu links wird deutlich, dass die Verfahren bei der Messung der linken Seite stärker korrelierten, als jene der rechten Seite. Die Ermittlung der statischen Okklusion mittels Shimstock-Folie bildet im Clustering eine eigene Gruppe und kann nicht in direkter Relation zu den anderen Messmethoden betrachtet werden.

\section{3 Übersicht der Verfahren}

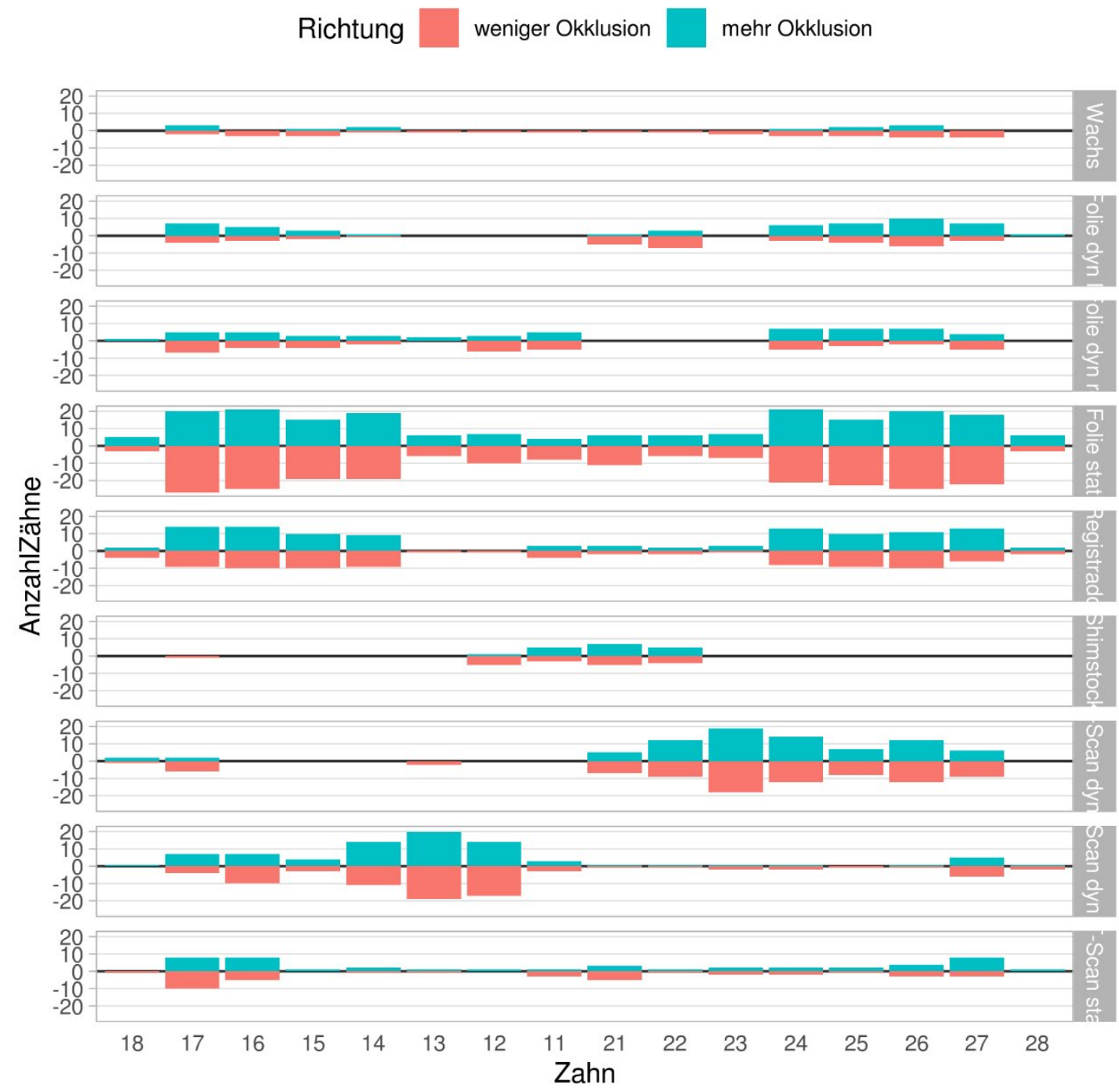

Abbildung 33: Erkannte Okklusionsveränderungen nach Messverfahren und Zahn. 
Die Abbildung 33 visualisiert die Okklusionsveränderungen gegenüber der 9-Uhr-Messung summiert über alle Probanden/-innen und beide Tage, die durch die verschiedenen Messmethoden erkannt wurden. Blaue Säulen deuten auf einen Zuwachs, rote auf eine Abnahme von Kontaktpunkten hin. Eine 5-prozentige Zu- oder Abnahme der Kontaktpunkte eines jeweiligen Zahnes wurde als Veränderung der Okklusion gedeutet. Hierbei ist augenscheinlich, dass die Messungen der dynamischen Okklusion mittels T-Scan-III-System eine Seite bevorzugten, was bei der Erhebung mittels Okklusionsfolie dynamisch weniger ausgeprägt war. Besonders die Schneidezähne zeigten eine geringere Seitenpräferenz. Des Weiteren ist auffällig, dass vor allem die Molaren einen höheren Änderungsgrad hinsichtlich der Kontaktpunktsituation aufwiesen. Die Registrierung mittels Shimstock-Folie bildete auch hier die Ausnahme, da lediglich im Frontzahnsegment Veränderungen festzustellen waren, die allerdings nicht das Signifikanzniveau erreichten. Aus der Abbildung 33 ist ebenfalls ersichtlich, dass die HS-ModellierwachsRegistrierung eher auf Änderungen im Molarensegment hinwies. Vergleichend kann zwischen der Shimstock-Folie und der HS-Modellierwachs-Registrierung festgestellt werden, dass die Shimstock-Folie tendenziell eher Veränderungen im Frontzahnsegment und annähernd keine im Molarensegment registrieren konnte, wohingegen HS-Modellierwachs annähernd keine Veränderungen im Frontzahnsegment tendenziell jedoch eher Änderungen im Molarensegment zeigen konnte. Dieser Zusammenhang wird ebenfalls in nachfolgender Abbildung 34 zur Kontaktpunktänderung deutlich. Für jedes Verfahren wurde die Anzahl der Kontaktpunktänderungen für jeden in der Registrierung beteiligten Zahn dargestellt. Hohe Balken weisen hierbei auf mehr-, niedrigere Balken auf weniger Änderung der jeweiligen Zähne im zugehörigen Messverfahren hin. 


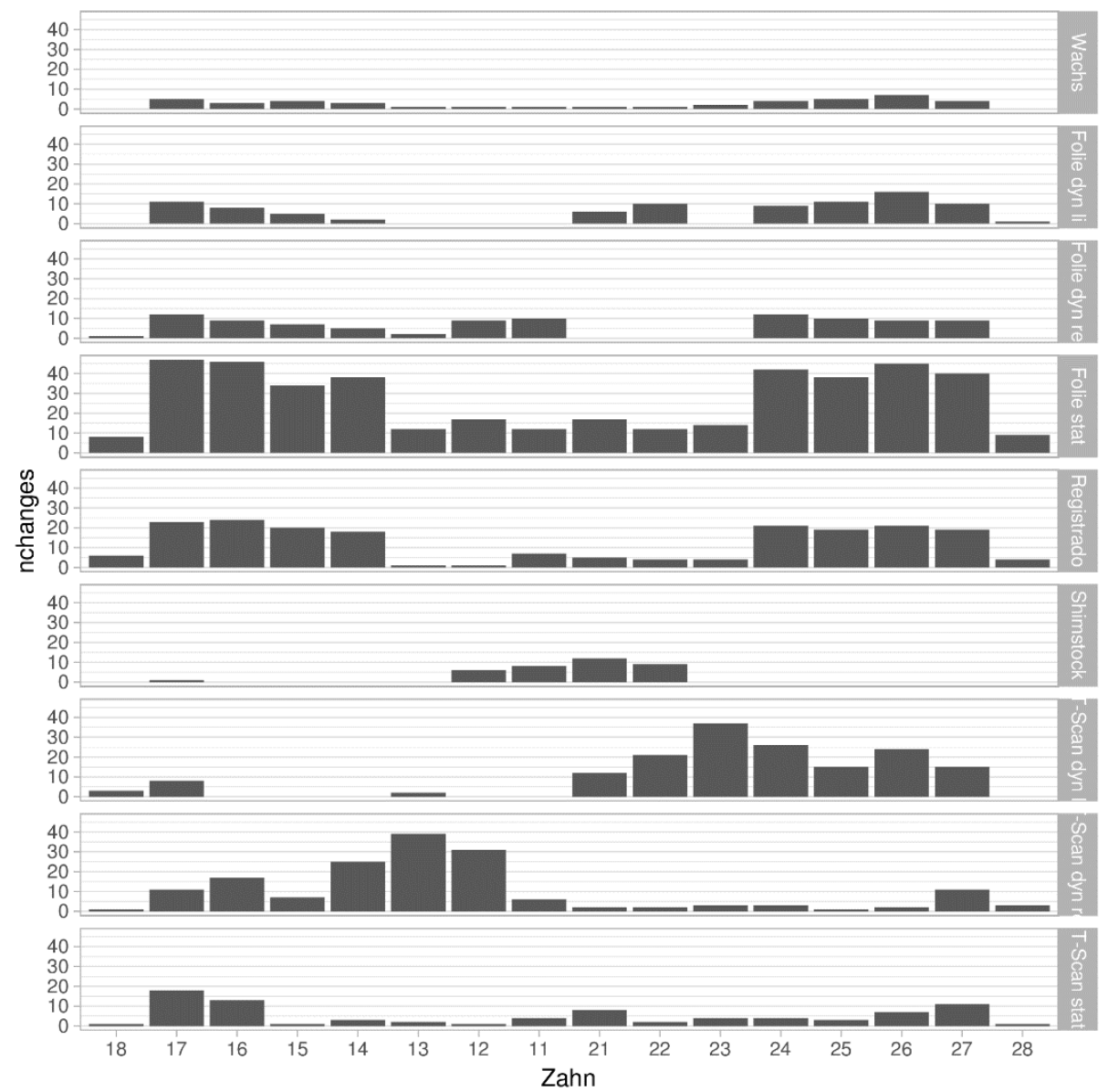

Abbildung 34: Änderungsausmaß nach Messverfahren und Zahn.

Um die deutlichen Unterschiede zwischen den verschiedenen Messmethoden formal statistisch zu testen, erfolgte die Modellierung der Veränderungen mittels logistischer Regression (Tab. A1-A4).

\subsection{Ergebnisse der Verfahren}

\subsubsection{HS-Modellierwachs}

Das Verfahren HS-Modellierwachs konnte im Mittel 4,1 Kontaktpunkte je Zahn mit einer Standardabweichung von $\pm 2,9$ nachweisen. Der Median lag unter Berücksichtigung von minimal 0 und maximal 17 bei 4 Kontaktpunkten (Tab. 5). 
Tabelle 5: Ermittelte Kontaktpunktanzahl je Zahn mittels Wachsregistrierung

\begin{tabular}{|c|c|}
\hline Verfahren HS-Modellierwachs & Kontaktpunktanzahl eines Zahnes \\
\hline Mittelwert \pm SD & $4,1 \pm 2,9$ \\
\hline Median (min; max) & $4(0 ; 17)$ \\
\hline
\end{tabular}

Tabelle 5 zeigt einen Ausschnitt der im Anhang befindlichen Tabelle A1 über die deskriptiven Werte der Studiendaten.

Dieses Verfahren konnte nach Auswertungen aller erhobenen Registrate das Signifikanzniveau von $\mathrm{p}<0,05$ erreichen. Die Wahrscheinlichkeit, an einem beliebigen Zahn keine Änderung zu registrieren, war jedoch signifikant größer, als eine Änderung zu registrieren. Die Randwahrscheinlichkeit eine Okklusionsänderung an einem beliebigen Zahn zu messen, lag bei diesem Verfahren bei 3,8\% (Tab. 6).

Tabelle 6: Prädizierte marginale Wahrscheinlichkeit (Wachsregistrat)

\begin{tabular}{|c|c|c|c|c|}
\hline Verfahren & Wahrscheinlichkeit & Konfidenzintervall & p-Wert & Signifikant \\
\hline Wachsregistrat & $3,8 \%$ & $(2,7 ; 5,2)$ & $<0,001$ & ja \\
\hline
\end{tabular}

Die geschätzte Randwahrscheinlichkeit aus dem im Anhang befindlichen Modell in Tabelle A3, eine Okklusionsänderung am Zahn zu messen (in \%). Die Spalte Konfidenzintervall enthält das zugehörige 95\%-Konfidenzintervall. Der aufgeführte p-Wert gibt den Test gegen $50 \%$ an.

\subsubsection{Okklusionsfolie $12 \mu \mathrm{m}$ zur Erhebung der statischen Okklusion}

Die Erhebung der statischen Okklusion anhand der Okklusionsfolie schwarz ergab einen Mittelwert von 3,7 Kontaktpunkten je Zahn bei einer Standardabweichung von \pm 2,7. Der Median lag hier bei 4 Kontaktpunkten, wobei ein Minimum von 0 und Maximum von 11 Kontakten je Zahn ermittelt wurde (Tab.7).

Tabelle 7: Ermittelte Kontaktpunktanzahl je Zahn in statischer Okklusion mittels Okklusionsfolie

\begin{tabular}{|c|c|}
\hline Okklusionsfolie statische Okklusion & Kontaktpunktzahl eines Zahnes \\
\hline Mittelwert \pm SD & $3,7 \pm 2,7$ \\
\hline Median (min; max) & $4(0 ; 11)$ \\
\hline
\end{tabular}

Tabelle 7 zeigt einen Ausschnitt der im Anhang befindlichen Tabelle A1 über die deskriptiven Werte der Studiendaten. 
Das Verfahren konnte nach Auswertungen aller erhobenen Registrate das Signifikanzniveau von $\mathrm{p}<0,05$ erreichen. Die Chance, an einem beliebigen Zahn keine Änderung zu registrieren, war jedoch signifikant größer, als eine Änderung zu registrieren. Die Randwahrscheinlichkeit eine Okklusionsänderung an einem beliebigen Zahn zu messen, lag bei diesem Verfahren bei $41,6 \%$ (Tab. 8).

Tabelle 8: Prädizierte marginale Wahrscheinlichkeit (Okklusionsfolie stat.)

\begin{tabular}{|c|c|c|c|c|}
\hline Verfahren & Wahrscheinlichkeit & Konfidenzintervall & p-Wert & Signifikant \\
\hline $\begin{array}{c}\text { Okklusionsfolie } \\
\text { stat. }\end{array}$ & $41,6 \%$ & $(37 ; 46,4)$ & 0,001 & ja \\
\hline
\end{tabular}

Die geschätzte Randwahrscheinlichkeit aus dem im Anhang befindlichen Modell in Tabelle A3, eine Okklusionsänderung am Zahn zu messen (in \%). Die Spalte Konfidenzintervall enthält das zugehörige 95\%-Konfidenzintervall. Der aufgeführte p-Wert gibt den Test gegen 50\% an.

\subsubsection{Okklusionsfolie $12 \mu \mathrm{m}$ zur Erhebung der dynamischen Okklusion nach links}

Die Registrierung der dynamischen Okklusion nach links mittels Okklusionsfolie rot ergab einen Mittelwert von 0,34 Kontaktpunkten und eine Standardabweichung von \pm 0,51 je Zahn. Der Median lag bei 0 ausgehend von minimal 0 und maximal 4 Kontaktpunkten (Tab. 9). Die Registrierung konnte front-/eckzahngeführte, unilateral geführte und bilateral geführte dynamische Okklusionen feststellen (Daten nicht ausgewiesen).

Tabelle 9: Ermittelte Kontaktpunktanzahl je Zahn in dynamischer Okklusion nach links mittels Okklusionsfolie

\begin{tabular}{|c|c|}
\hline Okklusionsfolie dyn. Okklusion li. & Kontaktpunktzahl eines Zahnes \\
\hline Mittelwert \pm SD & $0,34 \pm 0,51$ \\
\hline Median (min; max) & $0(0 ; 4)$ \\
\hline
\end{tabular}

Tabelle 9 zeigt einen Ausschnitt der im Anhang befindlichen Tabelle A1 über die deskriptiven Werte der Studiendaten.

Tabelle 10 zeigt, dass das Verfahren nach Auswertungen aller erhobenen Registrate das Signifikanzniveau von $\mathrm{p}<0,05$ erreichen konnte. Die Chance, an einem beliebigen Zahn keine Änderung zu registrieren, war jedoch signifikant größer, als eine Änderung zu registrieren. Die Randwahrscheinlichkeit eine Okklusionsänderung an einem beliebigen Zahn zu messen, lag bei diesem Verfahren bei 8,1 \% (Tab. 10). 
Tabelle 10: Prädizierte marginale Wahrscheinlichkeit (Okklusionsfolie dyn. li.)

\begin{tabular}{|c|c|c|c|c|}
\hline Verfahren & Wahrscheinlichkeit & Konfidenzintervall & p-Wert & Signifikant \\
\hline $\begin{array}{c}\text { Okklusionsfolie } \\
\text { dynamisch li. }\end{array}$ & $8,1 \%$ & $(6,3 ; 10,3)$ & $<0,001$ & ja \\
\hline
\end{tabular}

Die geschätzte Randwahrscheinlichkeit aus dem im Anhang befindlichen Modell in Tabelle A3, eine Okklusionsänderung am Zahn zu messen (in \%). Die Spalte Konfidenzintervall enthält das zugehörige 95\%-Konfidenzintervall. Der aufgeführte p-Wert gibt den Test gegen 50\% an.

\subsubsection{Okklusionsfolie $12 \mu \mathrm{m}$ zur Erhebung der dynamischen Okklusion nach rechts}

Die Registrierung der dynamischen Okklusion nach rechts mittels Okklusionsfolie rot ergab einen Mittelwert von 0,38 Kontaktpunkten mit einer Standardabweichung von \pm 0,54 je Zahn. Der Median lag bei 0 ausgehend von minimal 0 und maximal 4 Kontaktpunkten (Tab. 11). Die Registrierung konnte front-/eckzahngeführte, unilateral geführte und bilateral geführte dynamische Okklusionen feststellen (Daten nicht ausgewiesen).

Tabelle 11: Ermittelte Kontaktpunktanzahl je Zahn in dynamischer Okklusion nach rechts mittels Okklusionsfolie

\begin{tabular}{|c|c|}
\hline Okklusionsfolie dyn. Okklusion re. & Kontaktpunktzahl eines Zahnes \\
\hline Mittelwert \pm SD & $0,38 \pm 0,54$ \\
\hline Median (min; max) & $0(0 ; 3)$ \\
\hline
\end{tabular}

Tabelle 11 zeigt einen Ausschnitt der im Anhang befindlichen Tabelle A1 über die deskriptiven Werte der Studiendaten.

Dieses Verfahren konnte nach Auswertungen aller erhobenen Registrate das Signifikanzniveau von $\mathrm{p}<0,05$ erreichen. Die Chance, an einem beliebigen Zahn keine Änderung zu registrieren, war jedoch signifikant größer, als eine Änderung zu registrieren. Die Randwahrscheinlichkeit eine Okklusionsänderung an einem beliebigen Zahn zu messen, lag bei diesem Verfahren bei $8,6 \%$ (Tab. 12). 
Tabelle 12: Prädizierte marginale Wahrscheinlichkeit (Okklusionsfolie dyn. re)

\begin{tabular}{|c|c|c|c|c|}
\hline Verfahren & Wahrscheinlichkeit & Konfidenzintervall & p-Wert & Signifikant \\
\hline $\begin{array}{c}\text { Okklusionsfolie } \\
\text { dynamisch re. }\end{array}$ & $8,6 \%$ & $(6,8 ; 10,9)$ & $<0,001$ & ja \\
\hline
\end{tabular}

Die geschätzte Randwahrscheinlichkeit aus dem im Anhang befindlichen Modell in Tabelle A3, eine Okklusionsänderung am Zahn zu messen (in \%). Die Spalte Konfidenzintervall enthält das zugehörige 95\%-Konfidenzintervall. Der aufgeführte p-Wert gibt den Test gegen 50\% an.

\subsubsection{Silikonregistrierung Registrado X-tra zur Erhebung der statischen Okklusion}

Das Bissregistrat mit Registrado X-tra zeigte im Mittel 4,5 Kontaktpunkte, bei einer Standardabweichung von $\pm 3,2$. Auch bei diesem Verfahren lag der Median bei 4, wobei minimal 0 und maximal 17 Kontaktpunkte registriert wurden (Tab. 13).

Tabelle 13: Ermittelte Kontaktpunktanzahl je Zahn in statischer Okklusion mittels Silikonregistrat

\begin{tabular}{|c|c|}
\hline Silikonregistrat & Kontaktpunktzahl eines Zahnes \\
\hline Mittelwert \pm SD & $4,5 \pm 3,2$ \\
\hline Median (min; max) & $4(0 ; 17)$ \\
\hline
\end{tabular}

Tabelle 13 zeigt einen Ausschnitt der im Anhang befindlichen Tabelle A1 über die deskriptiven Werte der Studiendaten.

Die Silikonregistrierung konnte nach Auswertungen aller erhobenen Registrate das Signifikanzniveau von $\mathrm{p}<0,05$ erreichen (Tab. 14). Die Chance, an einem beliebigen Zahn keine Änderung zu registrieren, war jedoch signifikant größer, als eine Änderung zu registrieren. Die Randwahrscheinlichkeit eine Okklusionsänderung an einem beliebigen Zahn zu messen, lag bei diesem Verfahren bei $18,4 \%$.

Tabelle 14: Prädizierte marginale Wahrscheinlichkeit (Silikon)

\begin{tabular}{|c|c|c|c|c|}
\hline Verfahren & Wahrscheinlichkeit & Konfidenzintervall & p-Wert & Signifikant \\
\hline Silikonregistrat & $18,4 \%$ & $(15,3 ; 21,8)$ & $<0,001$ & ja \\
\hline
\end{tabular}

Die geschätzte Randwahrscheinlichkeit aus dem im Anhang befindlichen Modell in Tabelle A3, eine Okklusionsänderung am Zahn zu messen (in \%). Die Spalte Konfidenzintervall enthält das zugehörige 95\%-Konfidenzintervall. Der aufgeführte p-Wert gibt den Test gegen 50\% an. 


\subsubsection{Kontaktfolie Hanel Shimstock-Folie $8 \mu \mathrm{m}$ zur Erhebung der statischen Okklusion}

Das Shimstock-Verfahren nimmt eine gesonderte Rolle ein, da hierbei nicht mit numerischen Größen (Anzahl der Kontaktpunkte), sondern der reinen Unterscheidung in + (starker Kontakt), +/- (schwacher Kontakt) und - (kein Kontakt) gearbeitet wurde. Die Okklusionsregistrierung zeigte mit dem Median „Plus“ bei minimaler Erfassung von „minus“ und maximaler von „plus“ eine Orientierung hinsichtlich des Vorhandenseins eines Kontaktes (Tab. 15).

Tabelle 15: Befunde des Zugtests mittels Kontaktfolie

\begin{tabular}{|c|c|}
\hline Kontaktfolie & Befund des Zugtests an 1.608 Zähnen \\
\hline- & $128(8,0 \%)$ \\
\hline \pm & $118(7,3 \%)$ \\
\hline+ & $1362(84,7 \%)$ \\
\hline
\end{tabular}

Tabelle 15 zeigt einen Ausschnitt der im Anhang befindlichen Tabelle A1 über die deskriptiven Werte der Studiendaten.

Die Shimstockregistrierung konnte nach Auswertungen aller erhobenen Registrate das Signifikanzniveau von $\mathrm{p}<0,05$ erreichen. Die Chance, an einem beliebigen Zahn keine Änderung zu registrieren, war jedoch signifikant größer, als eine Änderung zu registrieren. Die Randwahrscheinlichkeit eine Okklusionsänderung an einem beliebigen Zahn zu messen, lag bei diesem Verfahren bei 3,2\% (Tab. 16).

Tabelle 16: Prädizierte marginale Wahrscheinlichkeit (Kontaktfolie)

\begin{tabular}{|c|c|c|c|c|}
\hline Verfahren & Wahrscheinlichkeit & Konfidenzintervall & p-Wert & Signifikant \\
\hline Kontaktfolie & $3,2 \%$ & $(2,3 ; 4,6)$ & $<0,001$ & ja \\
\hline
\end{tabular}

Die geschätzte Randwahrscheinlichkeit aus dem im Anhang befindlichen Modell in Tabelle A3, eine Okklusionsänderung am Zahn zu messen (in \%). Die Spalte Konfidenzintervall enthält das zugehörige 95\%-Konfidenzintervall. Der aufgeführte p-Wert gibt den Test gegen 50\% an.

\subsubsection{T-Scan-III-System zur Erhebung der statischen Okklusion}

Die Erfassung der statischen Okklusion mittels T-Scan-III-System zeigte einen Mittelwert an relativer Kaukraftbeteiligung je Zahn von 6,8 \% mit einer Standardabweichung von $\pm 5 \%$. Der Median dieser Registrate lag bei 5,9\% von minimal 0 und maximal $38 \%$ erfasster Beteiligung je Zahn (Tab 17). 
Tabelle 17: Ermittelte prozentuale Kaukraftbeteiligung der Gesamtkraft je Zahn in statischer Okklusion mittels T-Scan III

\begin{tabular}{|c|c|}
\hline T-Scan statisch & Prozentuale Beteiligung eines Zahnes \\
\hline Mittelwert \pm SD & $6,8 \pm 5$ \\
\hline Median (min; max) & $5,9(0 ; 38)$ \\
\hline
\end{tabular}

Tabelle 17 zeigt einen Ausschnitt der im Anhang befindlichen Tabelle A1 über die deskriptiven Werte der Studiendaten.

Das digitale Verfahren konnte nach Auswertungen aller erhobenen Registrate mit einem p-Wert von $<0,001$ das Signifikanzniveau von $\mathrm{p}<0,05$ erreichen. Die Chance, an einem beliebigen Zahn keine Änderung zu registrieren, war jedoch signifikant größer, als eine Änderung zu registrieren. Die Randwahrscheinlichkeit eine Okklusionsänderung an einem beliebigen Zahn zu messen, lag bei diesem Verfahren bei 7,5\% (Tab. 18).

Tabelle 18: Prädizierte marginale Wahrscheinlichkeit (T-Scan stat.)

\begin{tabular}{|c|c|c|c|c|}
\hline Verfahren & Wahrscheinlichkeit & Konfidenzintervall & p-Wert & Signifikant \\
\hline $\begin{array}{c}\text { T-Scan III } \\
\text { stat. }\end{array}$ & $7,5 \%$ & $(5,8 ; 9,6)$ & $<0,001$ & ja \\
\hline
\end{tabular}

Die geschätzte Randwahrscheinlichkeit aus dem im Anhang befindlichen Modell in Tabelle A3, eine Okklusionsänderung am Zahn zu messen (in \%). Die Spalte Konfidenzintervall enthält das zugehörige 95\%-Konfidenzintervall. Der aufgeführte p-Wert gibt den Test gegen 50\% an.

\subsubsection{T-Scan-III-System zur Erhebung der dynamischen Okklusion nach links}

Das T-Scan-III-System zeigte bei der Registrierung der dynamischen Okklusion nach links einen Mittelwert von $7 \%$ relativer Kaukraftbeteiligung je Zahn, bei einer Standardabweichung von $\pm 20 \%$. Der Median lag bei 0 von minimal $0 \%$ und maximal $100 \%$ (Tab. 19). Im Zuge der Registrierung konnten front-/eckzahngeführte, unilateral geführte und bilateral geführte dynamische Okklusionen festgestellt werden (Daten nicht ausgewiesen). 
Tabelle 19: Ermittelte prozentuale Kaukraftbeteiligung der Gesamtkraft je Zahn in dynamischer Okklusion nach links mittels T-Scan III

\begin{tabular}{|c|c|}
\hline T-Scan III dynamisch links & Prozentuale Beteiligung eines Zahnes \\
\hline Mittelwert \pm SD & $7 \pm 20$ \\
\hline Median (min; max) & $0(0 ; 100)$ \\
\hline
\end{tabular}

Tabelle 19 zeigt einen Ausschnitt der im Anhang befindlichen Tabelle A1 über die deskriptiven Werte der Studiendaten.

Tabelle 20 zeigt, dass mit dem digitalen Verfahren nach Auswertung aller erhobenen Registrate das Signifikanzniveau von $\mathrm{p}<0,05$ erreicht werden konnte. Die Chance, an einem beliebigen Zahn keine Änderung zu registrieren, war jedoch signifikant größer, als eine Änderung zu registrieren. Die Randwahrscheinlichkeit eine Okklusionsänderung an einem beliebigen Zahn zu messen, lag bei diesem Verfahren bei 15,1\% (Tab. 20).

Tabelle 20: Prädizierte marginale Wahrscheinlichkeit (T-Scan dyn. li.)

\begin{tabular}{|c|c|c|c|c|}
\hline Verfahren & Wahrscheinlichkeit & Konfidenzintervall & p-Wert & Signifikant \\
\hline $\begin{array}{c}\text { T-Scan III } \\
\text { links }\end{array}$ & $15,1 \%$ & $(12,4 ; 18,2)$ & $<0,001$ & ja \\
\hline
\end{tabular}

Die geschätzte Randwahrscheinlichkeit aus dem im Anhang befindlichen Modell in Tabelle A3, eine Okklusionsänderung am Zahn zu messen (in \%). Die Spalte Konfidenzintervall enthält das zugehörige 95\%-Konfidenzintervall. Der aufgeführte p-Wert gibt den Test gegen 50\% an.

\subsubsection{T-Scan-III-System zur Erhebung der dynamischen Okklusion nach rechts}

Die Registrierung der dynamischen Okklusion nach rechts zeigte anhand des T-Scan-IIISystems identische Werte (Tab. 21). Die Registrierung konnte wiederum front-/eckzahngeführte, unilateral geführte und bilateral geführte dynamische Okklusionen feststellen (Daten nicht ausgewiesen). 
Tabelle 21: Ermittelte prozentuale Kaukraftbeteiligung der Gesamtkraft je Zahn in dynamischer Okklusion nach rechts mittels T-Scan III

\begin{tabular}{|c|c|}
\hline T-Scan III dynamisch rechts & Prozentuale Beteiligung eines Zahnes \\
\hline Mittelwert \pm SD & $7 \pm 20$ \\
\hline Median (min; max) & $0(0 ; 100)$ \\
\hline
\end{tabular}

Tabelle 21 zeigt einen Ausschnitt der im Anhang befindlichen Tabelle A1 über die deskriptiven Werte der Studiendaten.

Das Verfahren konnte nach Auswertungen aller erhobenen Registrate das Signifikanzniveau von $\mathrm{p}<0,05$ erreichen. Die Chance, an einem beliebigen Zahn keine Änderung zu registrieren, war jedoch signifikant größer, als eine Änderung zu registrieren. Die Randwahrscheinlichkeit eine Okklusionsänderung an einem beliebigen Zahn zu messen, lag bei diesem Verfahren bei $15,2 \%$ (Tab. 22).

Tabelle 22: Prädizierte marginale Wahrscheinlichkeit (T-Scan dyn. re.)

\begin{tabular}{|c|c|c|c|c|}
\hline Verfahren & Wahrscheinlichkeit & Konfidenzintervall & p-Wert & Signifikant \\
\hline T-Scan III re. & $15,2 \%$ & $(12,5 ; 18,3)$ & $<0,001$ & ja \\
\hline
\end{tabular}

Die geschätzte Randwahrscheinlichkeit aus dem im Anhang befindlichen Modell in Tabelle A3, eine Okklusionsänderung am Zahn zu messen (in \%). Die Spalte Konfidenzintervall enthält das zugehörige 95\%-Konfidenzintervall. Der aufgeführte p-Wert gibt den Test gegen $50 \%$ an.

\section{5 Übersicht der wahrscheinlichen Veränderungen nach Verfahren}

Tabelle 23 zeigt die Gesamtübersicht der prädizierten marginalen Wahrscheinlichkeiten der verschiedenen Verfahren für die Okklusionsänderung eines beliebigen Zahnes.

Tabelle 23: Prädizierte marginale Wahrscheinlichkeiten der Verfahren für eine Okklusionsänderung an einem beliebigen Zahn

\begin{tabular}{|c|c|c|c|c|}
\hline Verfahren & Wahrscheinlichkeit & Konfidenzintervall & p-Wert & Signifikant \\
\hline Wachs & $3,8 \%$ & $(2,7 ; 5,2)$ & $<0,001$ & ja \\
\hline Folie stat. & $41,6 \%$ & $(37 ; 46,4)$ & $<0,001$ & ja \\
\hline Folie links & $8,1 \%$ & $(6,3 ; 10,3)$ & $<0,001$ & ja \\
\hline Folie rechts & $8,6 \%$ & $(6,8 ; 10,9)$ & $<0,001$ & ja \\
\hline Silikon & $18,4 \%$ & $(15,3 ; 21,8)$ & $<0,001$ & ja \\
\hline
\end{tabular}




\begin{tabular}{|c|c|c|c|c|}
\hline Verfahren & Wahrscheinlichkeit & Konfidenzintervall & p-Wert & Signifikant \\
\hline Kontaktfolie & $3,2 \%$ & $(2,3 ; 4,6)$ & $<0,001$ & nein \\
\hline $\begin{array}{c}\text { T-Scan III } \\
\text { stat. }\end{array}$ & $7,5 \%$ & $(5,8 ; 9,6)$ & 0,001 & ja \\
\hline $\begin{array}{c}\text { T-Scan III li. } \\
\text { T-Scan III re. }\end{array}$ & $15,1 \%$ & $(12,4 ; 18,2)$ & $<0,001$ & ja \\
\hline
\end{tabular}

Die geschätzten Randwahrscheinlichkeiten aus dem im Anhang befindlichen Modell in Tabelle A3, eine Okklusionsänderung am Zahn zu messen (in \%). Die Spalte Konfidenzintervall“ enthält das zugehörige 95\%-Konfidenzintervall. Der aufgeführte p-Wert gibt den Test gegen $50 \%$ an.

Tabelle 24 stellt die prädizierten marginalen Wahrscheinlichkeiten eine Okklusionsänderung an einem bestimmten Zahn zu sehen, zusammenfassend dar. In allen Verfahren war für jeden Zahn die Wahrscheinlichkeit für eine Veränderung in der Okklusionsmessung kleiner als $50 \%$. Die höchste Wahrscheinlichkeit in einer beliebigen Messung eine Änderung zu registrieren wurde in der Untersuchung an den Zähnen 18 und 28 ermittelt. Die niedrigste Wahrscheinlichkeit für eine Okklusionsänderung in der Registrierung konnte an den Front- und Eckzähnen ermittelt werden.

Tabelle 24: Prädizierte marginale Wahrscheinlichkeit für Okklusionsänderungen pro Zahn

\begin{tabular}{|c|c|c|c|c|}
\hline Zahn & Wahrscheinlichkeit & Konfidenzintervall & p-Wert & Signifikant \\
\hline 1 & $5,7 \%$ & $(4,5 ; 7,3)$ & $<0,001$ & ja \\
\hline 2 & $6,7 \%$ & $(5,3 ; 8,4)$ & $<0,001$ & ja \\
\hline 3 & $6,4 \%$ & $(5,1 ; 8,1)$ & $<0,001$ & ja \\
\hline 4 & $12,3 \%$ & $(10,2 ; 14,8)$ & $<0,001$ & ja \\
\hline
\end{tabular}




\begin{tabular}{|c|c|c|c|c|}
\hline Zahn & Wahrscheinlichkeit & Konfidenzintervall & p-Wert & Signifikant \\
\hline 5 & $11,6 \%$ & $(9,5 ; 14,1)$ & $<0,001$ & ja \\
\hline 6 & $14,8 \%$ & $(12,4 ; 17,6)$ & $<0,001$ & ja \\
\hline 7 & $15,1 \%$ & $(12,6 ; 17,9)$ & $<0,001$ & ja \\
\hline 8 & $18,1 \%$ & $(12,6 ; 25,3)$ & $<0,001$ & ja \\
\hline
\end{tabular}

Die geschätzten Randwahrscheinlichkeiten aus dem im Anhang befindlichen Modell in Tabelle A3, eine Okklusionsänderung am Zahn zu messen (in \%). Die Spalte Konfidenzintervall enthält das zugehörige 95\%-Konfidenzintervall. Der aufgeführte p-Wert gibt den Test gegen $50 \%$ an.

\subsubsection{Wahrscheinlichkeiten für Veränderungen in der Okklusionsmessung pro Kiefer}

Eine Veränderung der Okklusionsmessung pro Kiefer wurde immer dann gesehen, wenn mindestens zwei Zähne mit einer Änderung > 5 \% in einer Okklusionsmessung registriert wurden. Die Veränderungen wurden in Abhängigkeit von der Tageszeit, nämlich von 12 Uhr zu 9 Uhr und von 16 Uhr zu 9 Uhr, sowie zum Registrierverfahren modelliert.

Tabelle 25 verdeutlicht, dass die Wahrscheinlichkeit, eine Okklusionsänderung am Kiefer zu messen, für die Verfahren T-Scan III statisch, Kontaktfolie, Okklusionsfolie dynamisch links und rechts sowie HS-Modellierwachs jeweils signifikant kleiner als $50 \%$ war. Die Wahrscheinlichkeit, eine Okklusionsänderung am Kiefer mit den Verfahren Okklusionsfolie statisch, Registrado-X-tra-Silikonregistrierung, T-Scan III dynamisch links und rechts zu messen, war signifikant größer als $50 \%$.

Tabelle 25: Prädizierte marginale Wahrscheinlichkeit für Okklusionsänderungen pro Kiefer

\begin{tabular}{|c|c|c|c|c|}
\hline Verfahren & Wahrscheinlichkeit & Konfidenzintervall & p-Wert & Signifikant \\
\hline Wachs & $7,4 \%$ & $(3,3 ; 15,9)$ & $<0,001$ & ja \\
\hline Folie stat. & $98,8 \%$ & $(91,7 ; 99,8)$ & $<0,001$ & ja \\
\hline Folie links & $29,6 \%$ & $(19,9 ; 41,6)$ & 0,001 & ja \\
\hline Folie rechts & $29,6 \%$ & $(19,9 ; 41,6)$ & 0,001 & ja \\
\hline Silikon & $66,3 \%$ & $(54,1 ; 76,7)$ & 0,009 & ja \\
\hline Kontaktfolie & $13,8 \%$ & $(7,6 ; 23,8)$ & $<0,001$ & ja \\
\hline
\end{tabular}




\begin{tabular}{|c|c|c|c|c|}
\hline Verfahren & Wahrscheinlichkeit & Konfidenzintervall & p-Wert & Signifikant \\
\hline $\begin{array}{c}\text { T-Scan III } \\
\text { stat. }\end{array}$ & $20,3 \%$ & $(12,4 ; 31,4)$ & $<0,001$ & ja \\
\hline T-Scan III li. & $71,7 \%$ & $(59,8 ; 81,2)$ & 0,001 & ja \\
\hline T-Scan III re. & $67,7 \%$ & $(55,5 ; 77,8)$ & 0,005 & ja \\
\hline
\end{tabular}

Die geschätzten Randwahrscheinlichkeiten aus dem im Anhang befindlichen Modell in Tabelle A4, eine Okklusionsänderung am Kiefer zu messen (in \%). Die Spalte Konfidenzintervall enthält das zugehörige 95\%-Konfidenzintervall. Der aufgeführte p-Wert gibt den Test gegen 50\% an.

\subsubsection{Einfluss der Tageszeit auf die Wahrscheinlichkeit eine Veränderung zu messen}

Tabelle 26 beschreibt die Untersuchung des tageszeitlichen Einflusses der ausgewählten Messzeitpunkte auf die Chance eine Änderung der Okklusion pro Zahn mit einem Verfahren zu messen und basiert auf der logistischen Regression aus Tabelle A3, in der die Verfahren mit der HS-Modellierwachsmessung an Zahn 11 um 9 Uhr verglichen wurden.

Tabelle 26: Marginale Wahrscheinlichkeiten der Messzeitpunkte

\begin{tabular}{|c|c|c|c|}
\hline Verfahren & Zeitpunkt & marginale Ws. & Konfidenzintervall \\
\hline Wachs & 12 & 0,03401 & {$[0,02 ; 0,05]$} \\
\hline Wachs & 16 & 0,0412 & {$[0,03 ; 0,06]$} \\
\hline Folie links & 12 & 0,09098 & {$[0,07 ; 0,12]$} \\
\hline Folie links & 16 & 0,07054 & {$[0,05 ; 0,10]$} \\
\hline Folie rechts & 12 & 0,09659 & {$[0,07 ; 0,13]$} \\
\hline Folie rechts & 16 & 0,07611 & {$[0,05 ; 0,10]$} \\
\hline Folie statisch & 12 & 0,4171 & {$[0,36 ; 0,47]$} \\
\hline Folie statisch & 16 & 0,4154 & {$[0,15 ; 0,23]$} \\
\hline Silikon & 12 & 0,1884 & {$[0,14 ; 0,22]$} \\
\hline Silikon & 16 & 0,1787 & {$[0,02 ; 0,05]$} \\
\hline Kontaktfolie & 12 & 0,03397 & {$[0,02 ; 0,05]$} \\
\hline Kontaktfolie & 16 & 0,03035 & $0.47]$ \\
\hline
\end{tabular}




\begin{tabular}{|c|c|c|c|}
\hline Verfahren & Zeitpunkt & marginale Ws. & Konfidenzintervall \\
\hline T-Scan-III li. & 12 & 0,1363 & {$[0,11 ; 0,17]$} \\
\hline T-Scan-III li. & 16 & 0,1651 & {$[0,13 ; 0,21]$} \\
\hline T-Scan-III re. & 12 & 0,1671 & {$[0,13 ; 0,21]$} \\
\hline T-Scan-III re. & 16 & 0,1363 & {$[0,11 ; 0,17]$} \\
\hline T-Scan-III st. & 12 & 0,0761 & {$[0,05 ; 0,10]$} \\
\hline T-Scan-III st. & 16 & 0,07424 & {$[0,05 ; 0,10]$} \\
\hline
\end{tabular}

Die Spalte marginale Ws. enthält die geschätzte Randwahrscheinlichkeit eine Okklusionsänderung am Zahn zu messen. Die Spalte Konfidenzintervall enthält das zugehörige 95\%-Konfidenzintervall.

Tabelle 26 stellt die Randwahrscheinlichkeiten für eine Änderung in der Okklusionsmessung von $9 \mathrm{zu} 12$ Uhr und 9 zu 16 Uhr vergleichend dar. Unter Betrachtung des Verfahrens Okklusionsfolie $12 \mu \mathrm{m}$ zur Erhebung der statischen Okklusion konnte beispielsweise um 12 Uhr mit einer geschätzten Randwahrscheinlichkeit von 41,71 \% in der Stichprobe eine Änderung in der Okklusionsmessung festgestellt werden. Um 16 Uhr verhielt es sich mit einer Randwahrscheinlichkeit von 41,54 \% fast identisch. Es ist festzustellen, dass auch die anderen Registrierverfahren im Tageszeitvergleich sehr ähnliche Randwahrscheinlichkeiten für die unterschiedlichen Messzeitpunkte aufwiesen. So zeigte sich beispielsweise auch bei den Registrierverfahren für die dynamische Okklusion ein ähnliches Verhalten. Bei der Registrierung der Laterotrusion nach rechts mittels Okklusionsfolie $12 \mu \mathrm{m}$, konnte um $12 \mathrm{Uhr}$ mit einer Randwahrscheinlichkeit von 9,65\% in der Stichprobe eine Veränderung der Okklusionsmessung festgestellt werden. Um 16 Uhr belief sich die Randwahrscheinlichkeit auf 7,61\%. Auch hier war somit, wie in allen übrigen Verfahren, ein sehr ähnliches Verhalten über die Messzeitpunkte feststellbar.

Um einen möglicherweise signifikanten Unterschied der Messverfahren zu den Messzeitpunkten zu untersuchen, wurden adjuvant paarweise Vergleiche der Randwahrscheinlichkeiten aus Tabelle 26 durchgeführt (Tab. 27). 
Tabelle 27: Paarweiser Vergleich

\begin{tabular}{|c|c|c|c|c|}
\hline Verfahren & Kontrast & Relative Chance & Standardfehler & p-Wert \\
\hline Wachs & $12 / 16$ & 0,8191 & 0,2597 & 0,5291 \\
\hline Folie dyn. li. & $12 / 16$ & 1,319 & 0,2967 & 0,2189 \\
\hline Folie dyn. re. & $12 / 16$ & 1,298 & 0,2835 & 0,2325 \\
\hline Folie stat. & $12 / 16$ & 1,008 & 0,1297 & 0,9487 \\
\hline Silikon & $12 / 16$ & 1,067 & 0,1716 & 0,6872 \\
\hline Kontaktfolie & $12 / 16$ & 1,123 & 0,3826 & 0,7327 \\
\hline T-Scan dyn. li. & $12 / 16$ & 0,7982 & 0,1385 & 0,194 \\
\hline T-Scan dyn. re. & $12 / 16$ & 1,271 & 0,2201 & 0,1669 \\
\hline T-Scan stat. & $12 / 16$ & 1,027 & 0,2368 & 0,9076 \\
\hline
\end{tabular}

Paarweiser Vergleich der Randwahrscheinlichkeiten der Tageszeiten aus Tabelle 26.

Unter Berücksichtigung der p-Werte aller Registrierverfahren, konnte in keinem Verfahren das Signifikanzniveau von 5\% erreicht bzw. unterschritten werden (Tab. 26). Somit ist augenscheinlich, dass in keinem Verfahren in Bezug auf die Untersuchungszeitpunkte des Studienprozederes, ein signifikanter Unterschied zwischen den Messzeitpunkten beobachtet wurde. Für die Wahrscheinlichkeit, Okklusionsänderungen zu registrieren, bestand also zwischen den gewählten Messzeitpunkten kein signifikanter Unterschied, wenngleich das Ausmaß an Okklusionsänderungen der Einzelmessungen deutliche, signifikante Unterschiede zeigte.

\subsubsection{Die Relation der Verfahren zueinander}

Um die verwendeten Materialen abschließend auf Basis der statistischen Auswertung miteinander zu vergleichen, wurden die Methoden in Tabelle 28 basierend auf dem Modell der im Anhang befindlichen Tabelle A4 über die logistische Regression zur Okklusionsänderung pro Kiefer dargestellt. Die Wachsregistrierung zeigte zu den übrigen Registrierverfahren der statischen Okklusion signifikant andere Ergebnisse mit Ausnahme der Shimstock- und T-Scan-IIIRegistrierung, wohingegen die Registrierung der statischen Okklusion mittels Okklusionsfolie zu allen anderen Verfahren der statischen Okklusion immer signifikant andere Ergebnisse lieferte. Die Shimstock-Registrierung lieferte signifikant andere Ergebnisse als die Silikonregistrierung, jedoch nicht als die T-Scan-III-Registrierung. 
Ähnlich der Folienregistrierung, lieferte auch die Silikon-Registrierung im Vergleich zu allen anderen Verfahren der statischen Okklusion signifikant andere Ergebnisse. Die Ergebnisse der Verfahren zur Erhebung der dynamischen Okklusion unterschieden sich ebenfalls signifikant zueinander.

Tabelle 28: Paarweise Kontrasttests

\begin{tabular}{|c|c|c|c|}
\hline Verfahren & Relative Chance & Standardfehler & p-Wert \\
\hline Wachs / Folie stat. & 0,00099 & 0,0011 & $<0,001$ \\
\hline Wachs/ Silikon & 0,041 & 0,02 & $<0,001$ \\
\hline Wachs / Shimstock & 0,5 & 0,27 & 0,937 \\
\hline Wachs / T-Scan stat. & 0,32 & 0,16 & 0,374 \\
\hline Folie dyn. li. / Folie stat. & 0,0052 & 0,0054 & $<0,001$ \\
\hline Folie dyn. li. / Folie dyn. re & 1 & 0,36 & 1,000 \\
\hline Folie dyn. li. / T-Scan dyn. li. & 0,17 & 0,06 & $<0,001$ \\
\hline Folie dyn. re. / Folie stat. & 0,0052 & 0,0054 & $<0,001$ \\
\hline Folie dyn. re. / T-Scan dyn. re. & 0,2 & 0,072 & $<0,001$ \\
\hline Folie stat. / Silikon & 41 & 43 & 0,011 \\
\hline Folie stat. / Shimstock & 505 & 537 & $<0,001$ \\
\hline Folie stat. / T-Scan stat. & 317 & 333 & $<0,001$ \\
\hline Silikon / Shimstock & 12 & 5,1 & $<0,001$ \\
\hline Silikon / T-Scan stat. & 7,7 & 2,9 & $<0,001$ \\
\hline Shimstock / T-Scan stat. & 0,63 & 0,27 & 0,979 \\
\hline T-Scan dyn li / T-Scan dyn re & 1,2 & 0,43 & 1,000 \\
\hline T-Scan dyn li / T-Scan stat & 9,9 & 3,8 & $<0,001$ \\
\hline T-Scan dyn re / T-Scan stat & 8,2 & 3,1 & $<0,001$ \\
\hline
\end{tabular}

Paarweise Kontrasttests für die Verfahren der marginalen Effekte aus dem im Anhang befindlichen Modell in Tabelle A4 über die logistische Regression zur Okklusionsänderung pro Kiefer. 


\section{Diskussion}

\subsection{Material und Methode}

\subsubsection{Probanden/-innen}

Im Rahmen der durchgeführten Studie konnten 19 Probandinnen und Probanden, davon 14 weiblich und fünf männlich, untersucht werden. Das Alter des Probandenkollektivs lag im Mittel bei 30,8 \pm 4,8 Jahren. Die Studienpopulation setzte sich damit also mehrheitlich aus jungen erwachsenen Teilnehmerinnen zusammen. Aus Gründen der Homogenität und Vergleichbarkeit der Untersuchungsgruppe, war ein möglichst einheitliches Führungsmuster in der dynamischen Okklusion wünschenswert, das sich im allgemeinen Konsens, vor allem aber basierend auf den Erkenntnissen der umfangreich angelegten Studie von Panek et al. (2008) im Sinne der bilateral eckzahngeführten Okklusion mehrheitlich bei jungen Erwachsenen dieser Altersklasse (26\% der Teilnehmerinnen und Teilnehmer) feststellen ließ (Abduo et al. 2013; Al-Hiyasat und Abu-Alhaija 2004; Francová et al. 2015; Panek et al. 2008; Sreekumar et al. 2012; Wang et al. 2011). Die Ergänzung der Studienpopulation durch ältere Teilnehmerinnen und Teilnehmer wurde unter Berücksichtigung der Ergebnisse von Miyazaki et al. (2005) wie auch Panek et al. (2008) hinsichtlich einer mehrheitlich anzutreffenden Gruppenführung und damit gegebener Homogenität erwogen, aber aufgrund des häufig reduzierten Restzahnbestandes und der steigenden Prävalenz von Funktionsstörungen und anderen Pathologien bei älteren Menschen belassen (Miyazaki et al. 2005; Panek et al. 2008). Die Inhomogenität der Geschlechterverteilung der Untersuchungsgruppe ließ unter Berücksichtigung der Studie von Nadershah (2019), die eine Studienpopulation von 500 Probanden/-innen im Alter von 18 bis 89 Jahren gleicher Geschlechterverteilung aufwies, eine signifikant höhere Prävalenz vorhandener cranio-mandibulärer Dysfunktionen bei Frauen im Vergleich zu den männlichen Probanden vermuten ( 105 bzw. $42 \%$ der Teilnehmerinnen und 70 bzw. $28 \%$ der Teilnehmer). Die Geschlechterverteilung der vorliegenden Studie zeigte jedoch, dass dieser Zusammenhang hier nicht festgestellt werden konnte, was sich wohlmöglich durch die relativ geringe Anzahl der untersuchten Teilnehmerinnen und Teilnehmer erklären lässt. Vorliegende Funktionsstörungen im Sinne einer CMD führten aufgrund der Komplexität des Krankheitsbildes und der unsicheren Ursache sowie mangelnden Vergleichbarkeit mit funktionsgesunden Probandinnen und Probanden zum Studienausschluss (Ciancaglini et al. 2003; Türp et al. 2008). 
Unter Berücksichtigung der Untersuchungen von Aslam et al., Miyazaki et al., Namarig und Panek et al., die keinen signifikanten Zusammenhang zwischen der Art des Führungsmusters bzw. der Verteilung des Kontaktpunktmusters oder der -anzahl in Bezug auf das Geschlecht feststellen konnten, war die Beeinflussung der durchgeführten Untersuchung durch eine ungleiche Geschlechterverteilung ebenfalls nicht anzunehmen (Aslam et al. 2018; Miyazaki et al. 2005; Namarig SH 2015; Panek et al. 2008).

Die Studienteilnehmer wurden an zwei Untersuchungstagen im Abstand von 14 Tagen zu je drei Tageszeiten (9 Uhr, 12 Uhr und 16 Uhr) anhand von neun Registrierverfahren hinsichtlich Ihrer statischen und dynamischen Okklusion untersucht, um mögliche tageszeitabhängige Abweichungen der Okklusion festzustellen. Die Literatur zeigt zu diesem Studienfokus nur sehr wenige vergleichbare Studien, deshalb ist diesbezüglich vor allem die Untersuchung von Berry und Singh (1983) hervorzuheben. Da die habituelle Okklusion im Vergleich zu allen anderen Okklusionsmustern die mengenmäßig größte Anzahl an Kontaktpunkten aufweist (Posselt 1958; Singh 1970), und sich darüber hinaus in dieser Position eine balancierte Aktivität der Kaumuskulatur im Sinne einer Myozentrik feststellen ließe (Jankelson 1979), wurde die habituelle Interkuspidation in Hinblick auf tageszeitabhängige Abweichungen untersucht und die Kontaktpunktmuster mittels 47,5-52,5 $\mu \mathrm{m}$ messenden Okklusionspapieren bei 10 Probandinnen im Altersdurchschnitt von 18,2 Jahren registriert. Die Teilnehmerinnen wurden an drei separaten Untersuchungstagen morgens um 9 Uhr und nachmittags um 16 Uhr registriert. Zusätzlich wurde mittels Elektromyographie im Sinne der biofeedback therapy nach induzierter 30-minütiger Muskelrelaxation eine weitere Registrierung vorgenommen, um die muskelrelaxierte Situation der morgendlichen Messung zu imitieren. Leider wurden die Ergebnisse diesbezüglich nicht näher beschrieben. Berry und Singh kamen zu dem Ergebnis, dass eine signifikante Änderung des Kontaktpunktmusters der morgendlichen- zur nachmittäglichen Messung bei einem Signifikanzniveau von $\geq 2 \%$ festzustellen war. Hierbei zeigten sich in der morgendlichen Messung signifikant mehr Kontaktpunkte als nachmittags. Intensität und Trennschärfe der Kontakte nahm jedoch über den Tagesverlauf ab, was der schlafinduzierten Muskelrelaxation am Morgen zugeschrieben wurde, und seitens der Untersucher die Schlussfolgerung zuließ, anders als Jankelson (1979), einen Unterschied zwischen der myozentrischen und der interkuspidativen Position der Mandibel zu sehen (Berry und Singh 1983). Darüber hinaus wurde ebenfalls die Beeinflussung der Muskelaktivität durch im Tagesablauf üblichen, jedoch variablen physischen (Christensen 1981; Walder 1969) und psychischen (Berry und Wilmot 1977; Yemm 1969) Stress und die damit verbundene Auswirkung auf die Okklusion angeführt (Ai und Ishiwara 1968; Anderson und Picton 1957; Gillings et al. 1973; Graf und Zander 1963; Jankelson 1979; Ricketts 1969). 
Berry und Singh formulierten, dass sich die Okklusion und die okklusalen Kontakte im Laufe des Tages verändern und vom physischen Zustand der Kaumuskulatur und dem psychischen Zustand der Patientinnen und Patienten abhängen. Sie schlussfolgerten, dass die Auswirkungen auf die Philosophien der okklusalen Rehabilitation zu hinterfragen sind und dafür weitere Studien erforderlich sein, um die Bedeutung der Kontaktvariationen bei okklusalen Restaurationen, die zu verschiedenen Zeiten vorgenommen werden, zu bewerten (Berry und Singh 1983).

\subsubsection{Methode}

Nicht zuletzt um der Forderung von Berry und Singh (1983) Rechnung zu tragen, wurde im Rahmen dieser Studie ein Protokoll durchgeführt, an dem die Probandinnen und Probanden zu zwei Untersuchungstagen in 14-tägigem Abstand zu je drei Tageszeiten registriert wurden. Der Untersuchungstag sah hierbei eine Messung am Morgen um 9 Uhr, am Mittag um 12 Uhr und am Nachmittag um 16 Uhr vor, um die durch Berry und Singh beschriebene Kontaktpunktvariation über die Tageszeit genauer verfolgen zu können. Je Proband/-in wurden mittels fünf gängiger qualitativer und einem quantitativen Verfahren, 54 Registrate erstellt. Um eine möglichst optimale Vergleichbarkeit der Messungen zu realisieren, war ein definierter und reproduzierbarer Versuchsaufbau essenziell, dies sollte Variablen wie die Sitzposition während der Registrierung, die Registrierungsprozedur und - Unterweisung bis hin zur Auswertung der Messungen beinhalten. Angefangen bei der Sitzposition der Probanden/-innen, herrscht weitestgehender Konsens über die Beeinflussung der Körperhaltung auf die habituelle und dynamische Okklusion (Coelho et al. 2015; van't Spijker et al. 2011). Da sich die Studien bezüglich ihrer Ergebnisse hinsichtlich einer signifikanten Änderung der Okklusion ab einer Grad-definierten Entfernung von $90^{\circ}$ unterschieden, wurde in der vorliegenden Studie auf eine $90^{\circ}$-Sitzposition geachtet. Anschließend wurde bei der Positionierung der Probanden/-innen auf eine reproduzierbare Kopfhaltung geachtet, da nach Berücksichtigung der Studienlage bei Rotationen des Kopfes eine Veränderung der Kondylenposition und damit eine Veränderung der Okklusion zu erwarten war (Haralur et al. 2014; Katayama et al. 2018). Katayama et al. (2018) beobachteten eine signifikante Änderung der Kondyluslage bei Rotation des Kopfes in der Horizontalebene $\mathrm{ab} \geq 20^{\circ}$ von der Median-Sagittal-Ebene, weshalb die Ebenen-gerechte Ausrichtung gewahrt wurde. Zusätzlich wurde auch auf eine Positionierung des Kopfes in der Vertikalebene geachtet, da signifikante Veränderungen der sagittalen Kondylusposition bei vertikalen Kopfpositionen abseits der Frankfurter-Horizontal-Ebene festgestellt wurden (Ohmure et al. 2008; Preiskel 1965; Visscher et al. 2000). 
Ohmure et al. (2008) stellten bei einer bewussten Vorneigung des Kopfes um $10 \mathrm{~cm}$ Entfernung von der neutralen Kopfposition der Probanden/-innen unter Einhaltung der Frankfurter-Horizontal-Ebene eine signifikante Posteriorverlagerung der Kondylen in der Ruhelage fest, was durch die Dorsalflexion des Nackens zur Einnahme der vorgelehnten Position unter Einhaltung der Referenzebene erklärt wurde (Preiskel 1965). Unter Berücksichtigung dieser Erkenntnisse, wurde bei der Ausrichtung der vertikalen Kopfposition auf eine axiale Kopfhaltung mit Ausrichtung zur Frankfurter-Horizontal-Ebene geachtet. Die Beibehaltung dieser Position wurde bei der Durchführung sämtlicher Registrierungen gewahrt.

Wie bereits vorgestellt, spielten Konzentration (Berry und Wilmot 1977; Yemm 1969), Vigilanz (Berry und Singh 1983), Motivation und Tonus (Gurdsapsri et al. 2000; Hidaka et al. 1999; Imamura et al. 2015; Nishimori et al. 2017; Obara et al. 2014; Riise und Ericsson 1983; Tzakis et al. 1992) der Probanden/-innen bei der Registrierung in vergangenen Studien hinsichtlich der Okklusionsbeeinflussung eine wichtige Rolle. Hierbei handelt es sich jedoch um sehr komplexe und individuelle Parameter, deren Berücksichtigung im Studienprotokoll nicht möglich war. Die Zusammenhänge von Konzentration und Aufmerksamkeit in Bezug auf die Beeinflussung der Okklusion wurden bereits beschrieben, ohne jedoch auf die Zusammenhänge von Muskeltonus und Motivation einzugehen. Diesbezüglich wurden zahlreiche Studien publiziert, die den direkten Effekt erhöhter Kaukräfte auf das Okklusionsmuster mittels elektromyographischer Kontrolle partiell kontrovers aber überwiegend einstimmig beschrieben. Obara et al. (2014) konnten beispielsweise anders als Gurdsapsri et al. (2000) trotz erhöhter Kaumuskelaktivität gleichbleibende okklusale Verhältnisse feststellen und schrieben dies der klaren Anweisung ihren Probanden/-innen gegenüber zu. Die Studie sah die Registrierung des normalen, minimalen, leichten und starken Zubisses mittels Silikon vor. Jede Bissintensität erfolgte hierbei jedoch auf ein spezifisches Kommando, wodurch eine bessere Führung der Probanden/-innen mit stabilen okklusalen Kontaktpunktverhältnissen gegeben war. Die erhobenen Silikonregistrate der 12 Probanden/-innen wurden quantitativ mittels Transillumination (Biteeye), die bereits in einer vorhergegangenen Studie (2013) verwendet wurde, untersucht. Die Untersuchung von Gurdsapsri et al. (2000) verlief grundsätzlich ähnlich, jedoch wurden keine differenzierten Kommandos durchgeführt und 25 Probanden/-innen annähernd gleicher Geschlechterverteilung (12:13) untersucht. Anders als Obara et al, konnten Gurdsapsri et al. eine signifikante Kontaktpunktänderung des Okklusionsmusters bei zunehmender Kaukraft an Prämolaren und Molaren im Sinne einer Flächenvergrößerung feststellen, was von Hidaka et al. (1999) und später Imamura et al. (2015) und Nishimori et al. (2017) ebenfalls gezeigt wurde, wobei jedoch keine Änderung im anterioren Zahnsegment erkannt wurde. 
In der qualitativen Untersuchung durch Riise (1982) wurde ebenfalls eine mit der Bisskraft assoziierte Veränderung des Kontaktpunktmusters festgestellt, wobei jedoch entgegen der Feststellung von Gurdsapsri et al. (2000) und Nishimori et al. (2017) der vornehmliche Kontaktpunktzuwachs im anterioren Zahnsegment gesehen wurde. Die Registrierung erfolgte mit $8 \mu \mathrm{m}$ starken, einseitig färbenden Okklusionsfolien nach vormaliger Instruktion der Probanden/-innen. Unter Berücksichtigung der vorgestellten Studienlage und der betonten Erkenntnis, durch stärkere Kaukräfte veränderte Kontaktpunktverhältnisse vorzufinden, mussten dezidierte Anweisungen in das Studienprotokoll aufgenommen werden. So wurden unter Berücksichtigung der Erkenntnisse von Obara et al. (2014) für die Registrierung in statischer und dynamischer Okklusion zuvor beschriebene differenzierte Kommandos festgelegt und die Probanden/-innen instruiert, den Ausgangspunkt der habituellen Interkuspidation immer nur bis zur individuell empfundenen Maximalverzahnung ohne maximale Kraftentfaltung aufzusuchen. Zur Verifizierung dieser Position wäre eine zusätzliche Oberflächenelektromyographie nach Vorbild der vorgestellten Studien statthaft gewesen. Da dieses Instrument jedoch zur Untersuchung nicht zur Verfügung stand, musste von einer Verwendung abgesehen werden. Andererseits sollte anhand der Studie ein möglichst genaues Abbild des klinischen Prozederes erzeugt werden, um Erkenntnisse für den praktischen Arbeitsablauf zu gewinnen. Riise (1982) konnte im Rahmen der bereits vorgestellten Untersuchung auch ohne Elektromyographie ähnliche Erkenntnisse hinsichtlich der Kontaktpunktvarianz bei unterschiedlichen Kaukräften gewinnen, untersuchte jedoch mit 224 Teilnehmerinnen und Teilnehmern deutlich mehr Individuen. Die unmittelbaren Zusammenhänge von Kopf- und Körperhaltung, Aufmerksamkeit, Konzentration sowie physischer und psychischer Verfassung legen die Vermutung nahe, dass die Einbringung von Fremdmaterialien im Sinne der Prüfmittel selbst eine Beeinflussung des stomatognathen Systems und nicht zuletzt der Okklusion bedeuten. Dezidierte Literaturverweise sollen im Folgenden unter Berücksichtigung der Studienergebnisse der einzelnen Registrierverfahren diskutiert werden.

\subsubsection{Okklusionsfolie}

Zur Okklusionsregistrierung mittels Okklusionsfolie $12 \mu \mathrm{m}$ war eine initiale Trocknung der Zahnreihen nötig (Sharma et al. 2013), da sich die Markierungseigenschaft auf feuchten Zahnoberflächen drastisch verschlechterte. Dieser klinische Aspekt wurde bereits durch Anderson et al. (1993) und Gazit et al. (1986) im Rahmen ihrer Vergleichsuntersuchungen zur Verwendung von Kontakt- und Markierungsfolien beschrieben. Die Literatur lieferte in diesem Zusammenhang keine genauen Empfehlungen zur Durchführung der Trockenlegung. 
Die im klinischen Ablauf übliche Trocknung der Zahnreihen mittels Druckluft erwies sich als unzureichend, da das initial getrocknete Zahnsegment durch Luftverwirbelung und fortschreitende Salivation zur erneuten Benetzung der Oberflächen bei Trocknung der anderen Zahnsegmente führte. Aus diesem Grund wurde der Aufbiss auf ein Vlies-Tuch gewählt, um eine gleichzeitige Trocknung aller Zahnsegmente zu erreichen. Der Benetzungsgrad der Zahnoberfläche steht in direktem Zusammenhang mit der Zeichnungsfähigkeit der Okklusionsfolie $12 \mu \mathrm{m}$ : Je trockener die Zahnoberfläche, desto besser erwies sich das Zeichnungsverhalten der Folie (Reiber et al. 1989). Trotz verbesserter Vlies-Trockenlegung der Zahnoberflächen, konnten selten alle Kontaktpunkte zu einem Zeitpunkt dargestellt werden, was die Aussagekraft der klinischen Anwendung limitierte (Anderson et al. 1993; Gazit et al. 1986; Reiber et al. 1989).

Die fotografisch fixierten Kontaktpunktmuster wurden nach zuvor beschriebenen Kriterien ausgewertet. Hierbei war unbedingt auf das Vorhandensein von Schlifffacetten zu achten, da die markierende Folie vor allem an scharfkantigen Oberflächen zu falsch-positiven Markierungen neigte, was bereits durch Halperin et al. (1982), Gazit et al. (1986) und Anderson et. al (1993) beschrieben wurde. Die Autoren wiesen auf die fragliche Reliabilität der Methode im Vergleich zu anderen Prüfmitteln, wie zum Beispiel der Shimstock-Folie hin. Anders als die Okklusionsfolie, wies die Shimstock-Folie eine deutlich geringere Interrater-Variabilität auf (Anderson et al. 1993). Die markierende Eigenschaft der Artikulationsfolie muss differenziert betrachtet werden, da sie einerseits eine praktikable Methode darstellt klinisch Kontaktpunkte zu visualisieren, zum Anderen jedoch eben diese Markierungspunkte häufig fehlerhaft sind (Anderson et al. 1993; Gazit et al. 1986; Halperin et al. 1982). Anderson et al. wiesen explizit auch auf die Wechselwirkung des Speichels mit dem Farbträger dergestalt hin, dass er häufig zur Bildung eines Schmierfilmes beitrüge und eine korrekte Identifizierung der Kontaktpunkte erschwere (1993). Weiterhin wurde auch auf die Einflussnahme der verschiedenen Dimensionierungen von Okklusions- und Kontaktfolien auf den Biss hingewiesen. Halperin et al. untersuchten diesbezüglich 13 Okklusions- bzw. Kontaktfolien und -papiere in der Dimensionierung von 13-122 $\mu \mathrm{m}$ auf Verformbarkeit sowie Zerreisfestigkeit und kamen zu der Erkenntnis, dass okklusale Prüfmittel dieser Form dünner als $21 \mu \mathrm{m}$ sein sollten, um die Propriozeption des stomatognathen Systems nicht zu beeinflussen (Halperin et al. 1982). Die Studie von Anderson et al. verwendete mit dem Accufilm II (Parkell, Firmingdale, New York) eine beidseitig färbende Okklusionsfolie der Stärke $24 \mu \mathrm{m}$, die damit über der von Halperin et al. (1982) definierten Propriozeptionsgrenze lag, wodurch Anderson et al. schlussfolgerten, deutliche Vorteile in der $8 \mu \mathrm{m}$ messenden Shimstock-Folie zu sehen (1993). Forrester et al. führten eine annähernd identische Untersuchung wie Halperin et al. an 23 Probanden/-innen durch. 
Hierbei wurde neben der bereits erwähnten Okklusionsfolie Accufilm II des Kalibers $24 \mu \mathrm{m}$, Okklusionsseide $(60 \mu \mathrm{m})$, der T-Scan-III-Sensor $(100 \mu \mathrm{m})$ und Artikulationspapier $(202 \mu \mathrm{m}) \mathrm{mit}$ einer zusätzlichen Oberflächenelektromyographie untersucht, um den Effekt der verwendeten Prüfmittel auf die Muskelaktivität zu untersuchen (Forrester et al. 2011). Die Arbeitsgruppe kam hierbei zu dem Schluss, dass sowohl die Okklusionsfolie als auch die $60 \mu \mathrm{m}$ messende Okklusionsseide keinen signifikanten Einfluss auf die Muskelaktivität hatten und somit eher ein Fokus auf die Steifigkeit des verwendeten Registriermaterials gelenkt werden sollte, da der bedeutend dünnere T-Scan-III-Sensor im Vergleich zum $202 \mu \mathrm{m}$ messenden, weniger steifen Artikulationspapier, fast identische Muskelaktivitäten zeigte (Forrester et al. 2011). Zusammenfassend ist festzustellen, dass neben der Dimensionierung der Okklusionsfolie auch die Rigidität des Materials berücksichtigt werden muss, um Irritationen bei der Registrierung zu vermeiden.

Nach statistischer Auswertung der in der vorliegenden Studie durchgeführten Okklusionsmessungen konnte für die Registrierung der statischen wie auch dynamischen Okklusion ein signifikanter Unterschied der Ergebnisse zu den übrigen Verfahren der statischen und dynamischen Okklusion festgestellt werden. Die Wahrscheinlichkeit, keine Okklusionsveränderung pro Zahn festzustellen, war jedoch bei allen drei Messungen (statische Okklusion, dynamische Okklusion nach links und rechts) signifikant höher, als eine Änderung zu sehen. Hierbei ist jedoch hervorzuheben, dass die Wahrscheinlichkeit, in der Registrierung der statischen Okklusion eine Änderung zu sehen, deutlich höher war als in der Registrierung der dynamischen Okklusion. Die deutlich höhere prädizierte Randwahrscheinlichkeit, mittels Okklusionsfolie statisch eine Veränderung in der Okklusionsmessung pro Zahn von $>40 \%$ festzustellen verdeutlicht dieses Ergebnis. Die Registrierung der dynamischen Okklusion nach links und rechts belegte hierbei nur Wahrscheinlichkeiten von $<10 \%$. Hierbei ist anzumerken, dass Plasmans et al. (1988) die Befunde in der Einzelzahnuntersuchung mittels Okklusionsfolie deutlich besser reproduzieren konnten, als in der Befundung des gesamten Zahnbogens. Bei der Detektion möglicher Veränderungen in der Okklusion des gesamten Zahnbogens, konnte die Registrierung der statischen Okklusion mit einer deutlich höheren prädizierten Wahrscheinlichkeit von 98,8 \% eine Änderung feststellen, wohingegen dies bei der dynamischen Registrierung nach rechts und links nur mit einer 29,6 \%-igen Wahrscheinlichkeit der Fall war. In diesem Zusammenhang gilt es zu hinterfragen, ob die statische Okklusion per se variabler als die dynamische Okklusion ist, die mangelnde Reproduzierbarkeit der Registrierung hierfür verantwortlich ist (Anderson et al. 1993; Gazit et al. 1986; Plasmans et al. 1988) oder ein tageszeitabhängiger Zusammenhang zugrunde zu legen ist (Berry und Singh 1983). 
Ein primärer Unterschied der statischen und dynamischen Okklusion ist in der Biomechanik zu sehen. Wird die statische Okklusion im Sinne der habituellen Interkuspidation aufgesucht, bewegen sich die Kiefer initial ohne Kontakt zu den Zähnen aufeinander zu. Im Zeitraum der kontaktlosen Bewegung übernehmen die Kiefergelenke und vornehmlich der Musculus masseter und -temporalis die Führung und Koordination des Unterkiefers zum Oberkiefer (Okeson 2019). Diese Betrachtungsweise fordert den dringenden Hinweis, dass die Gelenkführung und Zahnführung unabhängig voneinander zu sehen sind und einander nicht direkt beeinflussen (Angel 1948; Okeson 2019; Ricketts 1950). Die Muskulatur spielt nachvollziehbarer Weise, aber initial in Bezug auf die Variabilität des Kontaktpunktmusters wohlmöglich eine entscheidende Rolle. Hierbei sei abermals auf die Zusammenhänge von, Aufmerksamkeit, Konzentration, Müdigkeit, Beanspruchung und Motivation hingewiesen (Ai und Ishiwara 1968; Anderson und Picton 1957; Berry und Wilmot 1977; Christensen 1981; Gillings et al. 1973; Graf und Zander 1963; Jankelson 1979; Ricketts 1969; Walder 1969; Yemm 1969). Unter Berücksichtigung der Variabilität der Muskelaktivität aufgrund verschiedenster Einflüsse, und der Tatsache, dass trotz der physiologischen Zusammenhänge des stomatognathen Apparates, Kiefergelenke und Zähne nicht immer in unmittelbaren Zusammenhang gesetzt werden müssen, erscheint die festgestellte Variabilität der statischen Okklusion nachvollziehbar. So wird der/die Proband/-in nach einer erholsamen Nachtruhe mit entspanntem Muskeltonus, wie durch Berry und Singh (1983) beschrieben, in der morgendlichen Registrierung einen anderen initialen Zahnkontakt aufweisen, als nach einem physisch und psychisch belastenden Arbeitstag mit eventuell verändertem Muskeltonus. Die Zahnreihen werden in beiden Fällen aus verschiedenen Initialpositionen in die Interkuspidation geführt, was einer „Rutschbewegung“ gleich kommt, die ein in der Ausprägung variables Kontaktpunktmuster erzeugt (Woda et al. 1979).

Zusätzlich muss auch die individuell applizierte Kaukraft der Probanden/-innen berücksichtigt werden, die trotz des definierten Kommandos nicht als konstant vorausgesetzt werden konnte. Zahlreiche bereits angeführte Studien belegten die Beeinflussung der Okklusion durch verschiedene Kaukräfte mittels Elektromyographie (Ferrario et al. 2002; Gurdsapsri et al. 2000; Hidaka et al. 1999; Imamura et al. 2015; Nishimori et al. 2017), die aus genannten Gründen nicht durchgeführt werden konnte. Andererseits konnten die Studien ohne elektromyographische Kontrolle ähnliche Aussagen bezüglich des Zusammenhangs von Kaukraft und Okklusion formulieren (McDevitt und Warreth 1997; Reiber und Müller 1994; Riise 1982; Riise und Ericsson 1983). Riise et al. konnten zusätzlich feststellen, dass junge Probanden/-innen unter 30 Jahren bei leichtem Kaudruck mehr Kontaktpunkte aufwiesen als ältere Probanden/-innen. Bei Ausübung höherer Kaukräfte waren diese Unterschiede jedoch nicht mehr feststellbar (Riise 1982). 
Die Arbeiten lieferten teilweise kontroverse Aussagen hinsichtlich der beteiligten Zahnsegmente (Frontzähne, Prämolaren und Molaren). Reiber und Müller (1994) stellten diesbezüglich ein Verhältnis von $1: 1,3: 2,1$ bei leichtem Zahnreihenschluss und zusätzliche Molarenkontakte bei deutlicher Kaukraftzunahme im Sinne des Pressens fest, was in der Tendenz ebenfalls später durch elektromyographisch-unterstützte Untersuchungen belegt wurde (Hidaka et al. 1999; Imamura et al. 2015; Nishimori et al. 2017) und den Ergebnissen von Ferrario et al. (2002), keine Unterschiede zu sehen, entgegen stand. Riise et al. (1983) erkannten bei deutlicher Kraftzunahme zusätzliche Frontzahnkontakte, was wiederum in sämtlichen vorgestellten Studien nicht gezeigt werden konnte (Ferrario et al. 2002; Gurdsapsri et al. 2000; Hidaka et al. 1999; Imamura et al. 2015; Nishimori et al. 2017). Es ist also festzustellen, dass eine Variabilität des Kontaktpunktmusters bei verschieden starken Zubissen beobachtet wird, jedoch vom Molarenzum Frontzahnsegment hin abnimmt. Ebenfalls muss in diesem Zusammenhang die teilweise widersprüchliche Studienlage betont werden (Kordaß und Lovrov 2013).

Die Ausgangssituation der dynamischen Okklusion war in der vorliegenden Studie bereits die Initialsituation der Bewegung und damit von Beginn an zahngeführt, was durchaus eine per se stabilere Ausgangssituation als die Ruheschwebelage ohne Zahnkontakt darstellte. Williamson und Lundquist (1983) untersuchten den Effekt verschiedener Kauoberflächen auf den Musculus masseter und -temporalis und kamen zu der Erkenntnis, dass bei den verschiedenen Okklusionskonzepten der Probanden/-innen ebenso verschiedene aber für das Okklusionskonzept typische Muskelaktivitäten zu beobachten waren. So wiesen Probanden/-innen mit einer bilateral balancierten Okklusion bei der dynamischen Okklusion Aktivitäten aller vier Muskeln auf. Wurden die Führungskontakte der Mediotrusionsseite beseitigt, also die bilateral balancierte Okklusion in eine unilateral balancierte Okklusion umgewandelt, übernahm das Muskelpaar (Musculus masseter, Musculus temporalis) der ipsilateralen Seite die Aktivität. Im Falle einer isolierten Eckzahnführung konnte darüber hinaus die solitäre Aktivität des Musculus temporalis der Laterotrusionsseite festgestellt werden. Okeson (2019) folgerte hieraus eine schonende Beanspruchung der Kaumuskulatur durch diesen okklusalen Parameter und ferner die Zusammenhänge zwischen Okklusion und Muskulatur (Belser und Hannam 1985; Manns et al. 1989; Manns et al. 1987; Miralles et al. 1988; Riise und Sheikholeslam 1984; Shupe et al. 1984). Unter Berücksichtigung dieser Ergebnisse muss die Inhomogenität der Probanden/-innen in Bezug auf das individuelle Führungsmuster herausgestellt werden, da neben der eckzahngeschützten Okklusion auch alle andern Führungsmuster, ausgenommen der bilateral balancierten Okklusion, festgestellt werden konnten. 
Setzt man die Erkenntnisse von Williamson und Lundquist mit den Ergebnissen der vorgestellten Studien über die variable Aktivität der Muskulatur durch verschiedenste Effekte in Zusammenhang, so ist per se immer dann eine höhere Varianz des Okklusionsmusters zu erwarten, wenn mehrere Muskeln verschiedener Aktivität an der Bewegung beteiligt sind. Interessanterweise konnte dies trotz der verschiedenen Führungsmuster in der vorliegenden Studie nicht gezeigt werden, da die dynamische Okklusion in der Folienregistrierung eine geringere Wahrscheinlichkeit für eine Okklusionsänderung im Vergleich zur Messung der statischen Okklusion erkennen ließ und dieser Zusammenhang auch nach Durchführung der vorgestellten Kontrasttests signifikant war. Somit ist die Ursache für stabilere okklusale Verhältnisse in der dynamischen Okklusion eventuell vornehmlich der Zahnführung zuzuschreiben, die den Muskelzug reguliert. Andererseits muss auch berücksichtigt werden, dass die dynamische Okklusion im Vergleich zum statischen Zubiss eine Bewegung höherer koordinativer Leistung darstellt, da die involvierten Muskelpaare anders als bei der statischen Okklusion unterschiedliche Aktivitäten zeigen müssen, um diese Grenzbewegung zu realisieren. In Zusammenhang mit den definierten Kommandos kann der/die Proband/-in also ganz bewusst immer wieder versucht haben die gleiche Bewegung zu vollführen, was eine bessere Reproduzierbarkeit des Bewegungsablaufes annehmen lässt (Obara et al. 2014).

In Bezug auf die gewählten Messzeitpunkte konnte kein signifikanter Unterschied festgestellt werden, was nicht bedeutet, dass kein tageszeitabhängiger Zusammenhang vorlag, sondern keine der gewählten Uhrzeiten mit einer signifikant höheren Wahrscheinlichkeit eine veränderte Okklusion zeigte. Dies unterstreicht die Ergebnisse von Berry und Singh (1983) hinsichtlich der Variabilität der Okklusion über den Tag hinweg, nicht aber in Bezug auf einen definierten Zeitraum. Unter Berücksichtigung der dargestellten Ergebnisse anderer Studien, muss die Okklusion im multikausalen Zusammenhang betrachtet werden. Es muss auch darauf hingewiesen werden, dass mittels eines qualitativen Verfahrens wie der Okklusionsfolie, trotz nicht gegebener Interraterdiskrepanzen, aufgrund der technischen Schwierigkeiten und der damit verbundenen erschwerten Realisierung reliabler und reproduzierbarer Ergebnisse (Kordaß und Lovrov 2013), eine tageszeitabhängige Abweichung der statischen und dynamischen Okklusion nicht sicher festgestellt werden konnte. 


\subsubsection{Shimstock-Folie}

Die Registrierung der statischen Okklusion mittels Shimstock-Folie sah die Untersuchung jedes einzelnen antagonistischen Zahnpaares bei Zubiss auf das vorab instruierte Kommando und dem damit verbundenen Erreichen der individuell-habituellen Interkuspidation (Qadeer 2017), nach dem vorgestellten Beispiel von Anderson et al. (1993) und Solberg (1986), vor. Die vorhandene Literatur befasst sich vornehmlich mit der Reliabilität der Untersuchung selbst (Anderson et al. 1993; Gazit et al. 1986), mit der Bisssperrung (Halperin et al. 1982) und dem Einfluss verschiedener Kaukräfte auf die Registrierung (Harper und Setchell 2002). Aufgrund der nicht vorhandenen Visualisierung der Kontaktpunktmuster, findet sich in der Literatur wenig in Bezug auf die Variabilität der Okklusion (Qadeer 2017). Lediglich Anderson et al. (1993) formulierten im Zusammenhang mit der Reliabilität der Ergebnisse von verschiedenen Untersuchern bei den einzelnen Zahnsegmenten eine mögliche Begründung ihrer Beobachtung vor allem im Front- und Eckzahnsegment deutliche Interratervariabilitäten zu sehen. Sie begründeten diesen Zusammenhang mit dem singulären und punktuellen Kontakt der Front- und Eckzähne, der wiederum durch die variable Unterkieferposition im Sinne einer bereits durch Woda et al. (1979) formulierten Rutschbewegung, Änderungen sowohl in der lateralen also auch antero-posterioren Richtung aufweisen kann (Anderson et al. 1993). Vor allem die Variabilität in der antero-posterioren Richtung ist hierbei von ausgesprochener Wichtigkeit, da so wohlmöglich die Erkenntnisse von Gurdsapsri et al. (2000), bei 25,3 \% der untersuchten Frontzähne von 25 Probanden/-innen keinen Frontzahnantagonismus festzustellen, erklärt werden können. Frühere Erkenntnisse von Beyron (1964), der bei 75 \% der untersuchten 46 Probanden/-innen im Alter von 15-24 Jahren (11 weiblich, 35 männlich) und McNamara und Henry (1974) die bei $40 \%$ der untersuchten 15 Probanden im Alter von 16-17 Jahren keinen Frontzahnantagonismus feststellen konnte/-en, unterstreichen diese Erkenntnis.

Harper und Setchell (2002) untersuchten anhand eines Laborversuchs den Einfluss verschiedener simulierter Kaukräfte von 0-400 Gramm in Zusammenhang mit einer schrittweise simulierten Bisssperrung von 0-8 $\mu \mathrm{m}$ und ermittelten die aufzubringenden Zugkräfte, um eine $8 \mu \mathrm{m}$ messende Shimstockfolie aus dem Antagonismus zwischen einer polierten Amalgamfüllung und einem intakten Molarenhöcker herauszuziehen. Sie kamen zu der Erkenntnis, dass die Zugkräfte linear mit Steigerung der simulierten Kaukraft und parallel simulierter Bisssperrung von 0-6 $\mu \mathrm{m}$ anstiegen und sich die Zugkräfte im Bereich von 0-4 $\mu \mathrm{m}$ Sperrung nicht signifikant unterschiedlich auf die Zugkräfte auswirkten. Ab einer Bisssperrung von $6 \mu \mathrm{m}$ sank die aufzuwendende Zugkraft, allerdings nicht in hohem Ausmaß, wohingegen die Zugkräfte bei $8 \mu \mathrm{m}$ unter 12 Gramm betrugen. 
Es wurde geschlussfolgert, dass Bisssperrungen bis $6 \mu \mathrm{m}$ nicht unterschieden werden konnten und eine Sperrung von $8 \mu \mathrm{m}$ immer noch einen leichten Kontakt bestimmen ließ. Das Vorhandensein bzw. die Abwesenheit eines Kontaktes ließ sich auch in der vorliegenden Studie klar nachweisen, dennoch konnte keine Aussage über die Lokalisation und Anzahl der Punkte getroffen werden, was sich für den dargestellten Untersuchungsfokus als ungünstig erwies. Zusätzlich musste der Untersucher selbst entscheiden, unter welchem Kraftaufwand die Folie unter Zugspannung versetzt wurde, was hinsichtlich der Genauigkeit des Prüfmittels zu kritisieren ist (Qadeer 2017). Da die Registrierung nur dichotome Aussagen erlaubte, bildete die Kontaktfolienregistrierung in der statistischen Auswertung eine separate Gruppe. Die schlichte Feststellung eines vorhandenen oder nicht vorhandenen Kontaktes zeigte sich in der vorliegenden klinischen Studie mit dem Ziel, tageszeitabhängige Änderungen oder einfache Änderungen festzustellen, als unzureichend präzise. Von 1608 untersuchten antagonistischen Zahnpaaren konnte bei $8 \%$ bzw. 128 Zahnpaaren ein Kontakt ausgeschlossen und bei 7,3 \% bzw. 118 Zahnpaaren ein unsicherer Befund festgestellt werden. Bei 1362 untersuchten Zahnpaaren also 84,7 \% konnte ein antagonistischer Kontakt festgestellt werden, wobei auch in dieser Registriermethode eine Variabilität der applizierten Kaukraft nicht ausgeschlossen werden konnte (Qadeer 2017). Legt man die Erkenntnisse von Harper und Setchell (2002) sowie Schelb und Kaiser (1985) zugrunde, so müssten die registrierten unsicheren Kontakte ebenfalls zu den Nicht-Vorhandenen gezählt werden, da sie im Bereich von $8 \mu \mathrm{m}$, also der Dimensionierung des Prüfmittels lagen und damit definitiv keine Kontaktpunktbeziehung belegten. Die Verteilung der positiven und negativen Kontaktrelationen wurde nicht ausgewertet, dennoch fanden sich im Rahmen der Registrierung negative Kontakte in konzentrierter Form im Frontzahnsegment, was prinzipiell die Erkenntnisse der Studien von Gurdsapsri et al. (2000), Anderson et al. (1993), Beyron (1954) und McNamara und Henry (1974) unterstreicht. Hinsichtlich der Wahrscheinlichkeit, sowohl Änderungen der Okklusion pro Zahn als auch pro Kiefer festzustellen, erwies sich die Kontaktfolienregistrierung mit einem Wert von $<5 \%$ pro Zahn und 13,8 \% pro Kiefer zusammen mit der Wachsregistrierung als Methode mit geringster Wahrscheinlichkeit zur Detektion von eventuellen Änderungen, was als deutliche Limitation in diesem Zusammenhang zu bewerten ist. Abschließend ist jedoch zu betonen, dass die Shimstock-Folie neben der Silikonregistrierung das Prüfmittel mit der geringsten Bisssperrung abbildete und nach Berücksichtigung der Studienlage damit die geringste Beeinflussung auf die Kaumuskulatur ausübte (Forrester et al. 2011; Halperin et al. 1982). 
Anders als sämtliche in dieser Untersuchung verwendeten Prüfmittel, die eine vornehmliche Änderung im Prämolaren und Molarensegment registrierten, zeigte die Shimstock-Registrierung den höchsten Änderungsgrad im Frontzahnbereich, was unter Berücksichtigung der Studien von Gurdsapsri et al. (2000), Beyron (1964) und McNamara und Henry (1974) und der damit zugrunde gelegten Begründung als nachvollziehbar erscheint. Die Tatsache, vornehmlich keine Änderungen im Seitenzahnsegment erkannt zu haben, macht jedoch unter Berücksichtigung der Studien von Hidaka et al. (1999), Imamura et al. (2015) und Nishimori et al. (2017) die Limitation des Prüfmittels deutlich.

\subsubsection{HS-Modellierwachs}

Versuchsaufbau, Durchführung und Auswertung der Bissregistrate mit HS-Modellierwachs glichen der bereits von Ehrlich und Taicher (1981) beschriebenen Methode, die maximale Interkuspidation klinisch zu untersuchen. Die erhobenen Wachsregistrate zeigten vor allem im Molarensegment im Vergleich zu den Prämolaren und Frontzähnen zahlreiche Kontaktpunkte im Sinne von annähernden und totalen Perforationen. Ehrlich und Taicher (1981) wie auch Millstein (1985) befundeten die erhobenen Registrate mittels Transillumination dergestalt, als dass sie totale Perforationen des Wachses als „Suprakontakt“, durchscheinende Areale mit intakter Wachsoberfläche als „Kontakt“ und einfache Zahnimpressionen als „annähernden Kontakt“ klassifizierten. Um einer genaueren Unterscheidung in „Kontakte“ und „keine Kontakte“ Rechnung zu tragen, wurden die anhand der Transillumination erkannten Kontaktpunkte in dieser Studie auf ihre Schichtdicke hin untersucht. Hierzu wurde ein Grenzwert für einen vorhandenen Kontakt bei einer maximalen Schichtstärke des Wachses von $\leq 20 \mu \mathrm{m}$ festgelegt. Die Festlegung dieser Grenze bezog sich auf die seitens Hützen et al. (2006) beschriebene und etablierte Methode zur Auswertung von Silikonregistraten.

Bei der kritischen Bewertung der Wachsregistrate ist die Untersuchung von Anup und Ahila (2011) zu berücksichtigen, die auf Aluminium- oder Kupferzusätze zur Modifikation der Materialeigenschaft von Registrierwachsen hinweist. Hierbei wurden verschiedene Fließtendenzen der Partikel von 2,5-22\% bei 37,5\% Mundtemperatur festgestellt, die eine hohe Anfälligkeit der Registrate bei Entnahme aus dem Mund hinsichtlich ungewünschter Verzerrungen und Verformungen bedeuten (Murray et al. 1999). Aus diesem Grund wurde im Rahmen der Materialauswahl auf ein Modellierwachs ohne metallische Zusätze zurückgegriffen, was jedoch zu Lasten der Grundstabilität des Registrates ging. Der hauptsächliche Vorteil des Wachses lag in der einfachen klinischen Anwendbarkeit und der Eigenschaft, bei Abkühlung schnell zu erhärten (Michalakis et al. 2004; Qadeer 2017). 
Umgekehrt muss jedoch berücksichtigt werden, dass ein Registrat dieser Materialeigenschaften ebenfalls einen spürbaren Widerstand bei Einnahme der habituellen Okklusion darstellt, obwohl die durchgeführte Erwärmung zu einer Erweichung des Materials führt (Michalakis et al. 2004; Murray et al. 1999). Damit nimmt es nach zuvor beschriebenen Studienergebnissen ebenfalls Einfluss auf die Okklusion, indem es den/die Probanden/-in trotz gegebener Kommandos zu verschieden starken Zubissen und damit verbundenen Muskelaktivitäten und Okklusionsmustern verleitet (Ferrario et al. 2002; Gurdsapsri et al. 2000; Hidaka et al. 1999; Imamura et al. 2015; Michalakis et al. 2004; Murray et al. 1999; Nishimori et al. 2017). Ferner ist bei der relativen Festigkeit des Wachses auch eine ungleichmäßige Auslenkung der Zähne im faserreichen Parodontalspalt und darüber hinaus eine veränderte Beanspruchung des Kiefergelenkes anzunehmen, die alleine durch Aufbiss eine Okklusionsänderung erzeugt (Millstein 1985; Murray et al. 1999). Aus dieser Erkenntnis wurde die Limitation des Registrates für Patientinnen und Patienten mit mobileren Zähnen oder reduzierter Kaukraft geschlussfolgert. Das in nur sehr geringem Umfang anhand der Wachsregistrierung festgestellte Änderungsausmaß des Frontzahnsegmentes in der vorliegenden Studie, lässt sich wohlmöglich aus genau diesem Zusammenhang erklären. Die vergleichsweise festen Wachsbisse könnten so durch den zusätzlichen Bedarf an Kaukraft zum Erreichen der habituellen Interkuspidation eine vollständige Autorotation des Unterkiefers verhindert haben, die wiederum für einen adäquaten Frontzahnantagonismus, sofern vorhanden, von Nöten gewesen wäre. Unter Berücksichtigung der Ergebnisse aus der Shimstockregistrierung, die genau gegensätzliche Ergebnisse lieferte, ist daher anzunehmen, dass die sperrige Wachregistrierung die Frontzahnsituation klinisch nicht korrekt abbildete. Weiterhin wurde auch die mangelnde Dimensionstreue des Registrates durch Schwindung, also Schrumpfung bei Abkühlung beschrieben, die eine zuverlässige Darstellung der okklusalen Beziehung nicht ermöglicht (Michalakis et al. 2004; Millstein 1985; Millstein et al. 1973; Yu und Lee 2010).

In Ergänzung an die fragliche Reliabilität der Materialeigenschaft, wurde die tatsächliche Befähigung des Materials hinterfragt, die feinen Kontaktpunktmuster über dünne Wachssepten adäquat abzubilden (Murray et al. 1999; Qadeer 2017). In diesem Zusammenhang wurde ebenfalls die Anfälligkeit der Registrierung, auf leichte Aufbisskräfte mit fehlender Perforation des Materials zu reagieren, beschrieben (Millstein 1985; Millstein et al. 1973). Zusätzlich beschrieben Millstein (1985) und Michalakis et al. (2004), dass sich neben der einwirkenden Kaukraft auch die Dauer der Okklusion auf die Komprimierbarkeit des Materials verzerrend auswirkte. 
Unter Berücksichtigung der Studienlage erweis sich die Wachsregistrierung auch in der vorliegenden Untersuchung, die sich der Analyse der statischen Okklusion im Zusammenhang mit der Tageszeit widmete, wenig dienlich. Hinsichtlich der Wahrscheinlichkeit, eine Okklusionsänderung pro Zahn zu messen, lag die Wachsregistrierung zwar - wie alle anderen Methoden auch - unter $50 \%$, zeigte aber mit einer Wahrscheinlichkeit von < $5 \%$ am wenigsten Kontaktpunktveränderungen.

Auch die Wahrscheinlichkeit, Okklusionsveränderungen in einer Messung im gesamten Zahnbogen zu sehen, war für die Wachsregistrierung mit 7,4\% am geringsten. Durch diese geringgradig festgestellten Veränderungen und des Nichtvorhandenseins eines Referenzwertes für okklusale Messungen, wurde die HS-Modellierwachs-Registrierung in diesem Versuch als Referenz für sämtliche andere Methoden herangezogen, um die Unterschiede der Messverfahren abzubilden. Es ist zu betonen, dass die qualitative Methode der Wachsregistrierung einer subjektiven Befundung des Untersuchenden unterliegt (Qadeer 2017). Quantifizierbare Methoden der computerunterstützten Transilluminationsauswertung, wie sie aus der Silikonregistrierung bekannt sind (Hützen et al. 2006; Obara et al. 2014; Obara et al. 2013) hätten zur Objektivierbarkeit der Befunde beigetragen, jedoch nicht die fraglichen und untersuchungsabhängigen Materialeigenschaften der Registrierung kompensieren können. Die Ergebnisse der durchgeführten Wachsregistrierung sind damit zusammenfassend kritisch zu hinterfragen und vor allem unter dem Aspekt eine Tageszeitabhängigkeit nachzuweisen, als wenig reliabel einzuordnen (Kordaß und Lovrov 2013; Michalakis et al. 2004; Millstein 1985; Millstein et al. 1973; Qadeer 2017; Yu und Lee 2010).

\subsubsection{Silikonregistrierung}

Versuchsaufbau, Durchführung und partiell die Auswertung der Silikonregistrate dieses Versuches, glich zahlreichen bereits durchgeführten Silikonuntersuchungen zur Erhebung der statischen Okklusion (Baba et al. 2000; DeLong et al. 2007; Durbin und Sadowsky 1986; Millstein 1984; Woda et al. 1987; Ziebert und Donegan 1979). Sämtliche angeführte Studien wiesen in den vergangenen Jahrzehnten bis heute auf die ausgezeichnete Registrierungseigenschaft der Silikone hin. DeLong et al. (2007) formulierten für dieses Material sogar eine höhere Genauigkeit bei Fragestellungen, die sonst der Kontaktfolie vorbehalten waren. Hinsichtlich des Anspruches an ein Registriermaterial, formulierten Michalakis et al. (2004) und Tejo et al. (2012), dass ein möglichst geringer Aufbisswiderstand vor dem Erreichen der maximalen Interkuspidation vorliegen solle, um keinen Einfluss auf die Zahnstellung bzw. die Okklusion auszuüben. Zusätzlich müsse die Dimensionsstabilität auch bei Kompression nach stattgefundener Abbindereaktion gegeben sein, um die tatsächliche Kontaktpunktrelation abzubilden. 
Des Weiteren müssen die Kontaktoberflächen präzise abgebildet werden, um sämtliche Kontaktareale genau registrieren zu können. Neben einer einfachen Handhabung und Befundung, müsse auch die Biokompatibilität gewährleistet sein. Unter Berücksichtigung dieser Attribute, untersuchten Wieckiewicz et al. (2016) 13 additionsvernetzende Silikone zur Bissregistrierung. Nach Applikation in einem standardisierten Versuchsaufbau und Erstellung gleichdimensionierter Probekörper wurden alle Proben in $23^{\circ} \mathrm{C}$ temperierter Umgebung nach 24 Stunden untersucht. Hierzu wurden die Probekörper der verschiedenen Silikone in eine Spannvorrichtung mit fünf Zentimeter Abstand zwischen den Einspannpunkten eingebracht. Die Apparatur versetzte die Probe mit einer konstanten Geschwindigkeit von 50 mm/Minute unter Zugspannung. Die aufzuwendende Kraft, wie auch der E-Modul und die maximale Elongation bis zum Zerreißen der Probe wurde computerunterstütz erfasst. Wieckiewicz et al. (2016) schlussfolgerten, dass auf Basis der aktuellen Studienlage die Höhe des E-Moduls und der Zerreißfestigkeit maßgeblich für die Qualität der okklusalen Registrierung seien. Die Silikone Registrado X-tra und Stonebite zeigten unter allen getesteten gängigen Materialien die besten E-Moduli und die höchste Zerreißfestigkeit, wie auch die höchste Übereinstimmung mit den seitens Tejo et al. (2012) und Michalakis et al. (2004) formulierten Anforderungen an okklusale Prüfmittel. Die exzellente Materialeigenschaft der Silikone wurde bereits in zahlreichen Studien bezüglich kraftinduzierter Veränderungen der Okklusionsmuster betont (Gurdsapsri et al. 2000; Hidaka et al. 1999; Imamura et al. 2015; Nishimori et al. 2017; Obara et al. 2014; Obara et al. 2013; Woda et al. 1987). Im Vergleich zu den anderen in dieser Studie verwendeten Registriermaterialien zur Erhebung der statischen Okklusion, nahm das Silikon durch die plastisch-verformbare Initialphase so gut wie keinen Einfluss auf die Okklusion selbst (Millstein 1985; Murray et al. 1999) oder die Muskelaktivität (Hidaka et al. 1999; Imamura et al. 2015; Nishimori et al. 2017). Auch gegenüber speichelbenetzter Zahnoberflächen zeigte sich das Material unempfindlich (Wieckiewicz et al. 2016). Nach abgeschlossener Abbindereaktion konnte das Registrat sicher und ohne Deformationen aus der Mundhöhle entnommen und analysiert werden. Hinsichtlich der Analyse von Silikonregistraten stehen sich zwei etablierte aber in Hinblick auf die Reliabilität deutlich unterscheidende Methoden gegenüber. Beiden Methoden liegt die Transillumination zu Grunde, wobei die Durchführung der Befundung den wichtigen Unterschied darstellt. Die etablierte Praxis stellt hierbei die optische und subjektive Beurteilung des Registrates vor einer Lichtquelle durch den Behandelnden dar (Dinçer et al. 2003; Ehrlich und Taicher 1981; Korioth 1990; Millstein 1985; Woda et al. 1987). 
Obwohl die Befundung anhand der bloßen Begutachtung klinisch einfach, schnell und sicher umzusetzen ist, wird ihr seit jeher durch den subjektiven und dadurch qualitativen Charakter mangelnde Reliabilität und Validität testiert (Kordaß und Lovrov 2013), was per se keine Kritik an der tatsächlichen Registrierung der individuellen Okklusion zulässt. Dieser Schwierigkeit wurde im Rahmen der computerunterstützten Transilluminationsverfahren begegnet, die es erlauben quantitativ und damit reliabel die Informationen der Silikonregistrate auszuwerten (Gurdsapsri et al. 2000; Hidaka et al. 1999; Hützen et al. 2006; Imamura et al. 2015; Nishimori et al. 2017; Obara et al. 2014; Obara et al. 2013). Ein weiterer Kritikpunkt der Registriermethode lag in der fraglichen Reproduzierbarkeit der Messungen auf Grund der individuell applizierten Kaukraft, der man mit der Verwendung einer Oberflächenelektromyographie begegnete und zugleich den Zusammenhang von Muskelaktivität und Änderungsgrad des Kontaktpunktmusters beschreiben konnte (Hidaka et al. 1999; Imamura et al. 2015; Nishimori et al. 2017). In diesem Zusammenhang beschrieben Obara et al. (2014) die Auswirkung definierter Kommandos bei der Registrierung in Bezug auf die festgestellte Muskelaktivität und konnten eine gute Reproduzierbarkeit ermitteln. Da in der vorliegenden Studie weder eine computergestützte Transillumination noch eine Oberflächenelektromyographie zur Verfügung standen, wurde die Registrierung nach dem Vorschlag von Obara et al. (2014) unter strengster Einhaltung der definierten Kommandos durchgeführt und anschließend qualitativ durch einen Behandler ausgewertet. Um den qualitativen Charakter der Befundung zu reduzieren, wurden intakte Silikonoberflächen nach dem Vorschlag von Hützen et al. (2006) vermessen und ab einer Schichtstärke von $\leq 20 \mu \mathrm{m}$ als Kontakt gewertet. Nach statistischer Auswertung der Silikonregistrierung konnte mit einer marginalen Wahrscheinlichkeit von 18,4 \% eine Veränderung in der Okklusionsmessung pro Zahn registriert werden. Damit zeigte diese Methode nach der statischen Okklusionsfolienregistrierung mit vergleichsweise hoher Wahrscheinlichkeit etwaige Änderungen an. Hinsichtlich der Wahrscheinlichkeit, eine Änderung der Okklusion im gesamten Zahnbogen festzustellen, konnte die Silikonregistrierung mit 66,3 \%-iger Wahrscheinlichkeit eine Änderung anzeigen. Unter Berücksichtigung der Ergebnisse von Gurdsapsri et al. (2000), Hidaka et al. (1999), Imamura et al. (2015) und Nishimori et al. (2017), vornehmliche Änderungsgrade an Prämolaren und Molaren festzustellen, konnte dies in der vorliegenden Studie ebenfalls erkannt werden. Von allen im Rahmen der vorliegenden Studie verwendeten Materialien, zeigte sich das Silikon am zuverlässigsten, da es sich weder negativ auf die Muskelaktivität, noch die Okklusion, noch die Qualität des Registrates in Wechselwirkung mit der oralen Umgebung auswirkte. 
In Hinblick auf die tageszeitabhängigen Abweichungen der Okklusion, konnte auch die Silikonregistrierung keinen signifikanten Zusammenhang zwischen den gewählten Untersuchungszeitpunkten und der okklusalen Änderung feststellen. Die marginale Wahrscheinlichkeit zur Registrierung einer Okklusionsänderung war damit zu jeder Uhrzeit gleichermaßen niedrig oder hoch. In diesem Zusammenhang wäre eine Ergänzung des Verfahrens mit quantitativen Hilfsmitteln wie der computergestützten Transillumination und einer Oberflächenmyographie dienlich gewesen, um die angenommenen Einflüsse der Tageszeit reliabler untersuchen zu können.

\subsubsection{T-Scan III}

Die vorhandene Literatur zur Anwendung des quantitativen Registrierverfahrens T-Scan III widmet sich vornehmlich der klinischen Anwendbarkeit gegenüber den konventionellen qualitativen Verfahren und besonders der Reliabilität, da sich bislang noch kein Registrierverfahren zur klinischen Anwendung unter diesem Anspruch bewährt hat (Gazit et al. 1986; Koos et al. 2010; Kordaß und Lovrov 2013; Millstein 1983; Reiber et al. 1989). Besonders im Vergleich quantitativer und qualitativer Verfahren wird der dringende Bedarf an reproduzierbaren und reliablen Prüfmitteln deutlich (Kerstein 2008a; Koos et al. 2010), die darüber hinaus durch ihre Materialbeschaffenheit keine nennenswerten Einflüsse auf die Okklusion selbst ausüben sollen (Forrester et al. 2011; Halperin et al. 1982; Kerstein 2001; Koos et al. 2010). Eine Vielzahl von Publikationen beschrieben in der Vergangenheit die deutliche Überlegenheit des T-Scan-Verfahrens im Vergleich zu konventionellen qualitativen Methoden, da vor allem die subjektive Befundung des Behandelnden vermieden werden konnte. Zusätzlich wurde die vorteilhafte und zuverlässige Möglichkeit beschrieben, Stör- und Vorkontakte sicher in der klinischen Anwendung detektieren zu können, den Aufbau der maximalen Interkuspidation im Bereich von einer hundertstel Sekunde nachvollziehen zu können, die Kraftverteilung beider Quadranten, wie auch des anterioren und posterioren Zahnsegmentes in Relation beurteilen zu können, eine evidente Auskunft über okklusale Kraftdiskrepanzen im Zahnbogen zu erhalten und die Möglichkeit, die Kraftverteilung und sichere Lokalisation der applizierten Kontaktfläche eines jeden Zahnes im Zahnbogen feststellen zu können (Afrashtehfar und Qadeer 2016; Carey et al. 2007; Kerstein 2010; Kerstein 2008a, 2008b; Kerstein et al. 2006; Kerstein 2004; Kerstein 1993; Kerstein und Grundset 2001; Kerstein und Radke 2012; Kerstein und Wilkerson 2001; Kerstein und Wright 1991; Mahony 2005; Qadeer et al. 2012). Koos et al. (2010) untersuchten den TScan III unter dem Aspekt der Messgenauigkeit und Reproduzierbarkeit, indem sie eine Studienpopulation bestehend aus 19 Teilnehmerinnen und 23 Teilnehmern im Alter zwischen 20 und 30 Jahren der Okklusionsregistrierung unterzogen. Jeder Teilnehmende wurde anhand von 5 Kauzyklen zu je 6 Messungen mittels zwei Sensorfolien untersucht. 
Jede Untersuchung sah also einen fünfmaligen Zubiss auf die Sensorfolie in habitueller Position vor. Zwischen den Untersuchungen wurde das Gerät aus der Mundhöhle entfernt und neu positioniert. Der Folienwechsel wurde nach drei Aufzeichnungen, also 15 Aufbissen vorgenommen. Das Studiendesign berücksichtigte neben der Reproduzierbarkeit der Messungen im gleichen Registrierungszeitraum, die möglichen Auswirkungen eines Sensorwechsels, der Sensorverformung und der Neupositionierung auf die Reliabilität der Ergebnisse. Zusätzlich untersuchte die Arbeitsgruppe 65 Folien mit einer Mikrometerschraube bezüglich ihrer tatsächlichen Dimension von $100 \mu \mathrm{m}$.

Ähnliche Versuche von Garrido Garcia et al. (1997) und Gonzalez Sequeros et al. (1997) zeigten bereits anhand des T-Scan II bei der Analyse von vier aufeinanderfolgenden Messungen eine Übereinstimmung von 90,3 \% und hinsichtlich der Kontaktpunktvarianz eine Abweichung von maximal drei Zähnen. Maness und Podoloff konnten anhand der Registrierung von 93 adulten Patientinnen und Patienten mittels T-Scan II gleichwertige Ergebnisse in Bezug auf qualitative Verfahren feststellen (Maness et al. 1987; Maness und Podoloff 1989). Vor dem Aspekt der zuvor von verschiedenen Autoren publizierten Schwachpunkte in Bezug auf Genauigkeit und Reproduzierbarkeit der T-Scan-Generationen I und II (Dees et al. 1992; Scholz et al. 1991; Setz und Geis-Gerstorfer 1990a; Tschernitschek et al. 1990), untersuchten Koos et al. (2010) damit die dritte Generation auch in Hinblick auf die Beseitigung der bekannten Mängel. Hinsichtlich der prozentualen Kraftverteilung pro Zahn in Relation zur applizierten Gesamtkraft, konnte eine Verteilung von 0 bis $41 \%$ über alle Teilnehmerinnen und Teilnehmer festgestellt werden. Ähnlich zeigte sich die im Rahmen der vorliegenden Studie erhobene relative Kaukraft je Zahn, mit einem ermittelten Bereich von 0 bis 38 \%. Zusätzlich konnte in der Studie von Koos et al. die häufige Abwesenheit von Kontakten im Bereich der seitlichen Schneidezähne, gefolgt von den mittleren Schneidzähnen und Eckzähnen festgestellt werden. Die vorliegende Studie erkannte für die betroffenen Zähne lediglich mit einer Wahrscheinlichkeit von 5,7 bis 6,7 \% eine Änderung der Kontaktpunkte zwischen den Messungen, was jedoch keine Aussage über die Quantität der vorhandenen- und nicht vorhandenen Kontakte zuließ. Ein ähnlicher Zusammenhang ließ sich in Bezug auf die Beobachtung seitens Koos et al. über die vornehmliche Kraftkonzentration auf das Molaren- und Prämolarensegment von 30 bis $41 \%$ ziehen. Die vorliegende Studie stellte im beschriebenen Areal unter Berücksichtigung aller Verfahren mit 12,3 bis 18,1 \% zu einer deutlich höheren Wahrscheinlichkeit Änderungen fest, was sich mit den Erkenntnissen von Hidaka et al. (1999), Imamura et al. (2015) und Nishimori et al. (2017) vereinbaren lässt. Koos et al. stellten einen Mittelwert von 6,9\% der maximalen Kaukraft mit einer Standardabweichung von 6,3 \% aller gemessenen Werte pro Zahn fest. 
Die Vorliegende Studie konnte mit einem Mittelwert von 6,8 \% und einer Standardabweichung von $5 \%$ zu sehr ähnlichen Ergebnissen kommen. Koos et al. (2010) konnten über die durchgeführten 30 Messungen keine statistisch signifikanten Abweichungen der erhobenen Werte zueinander feststellen und somit Messungenauigkeiten durch Sensorverformungen bei mehrmaliger Verwendung ausschließen. Zusätzlich hatte auch der vorgenommene Folienwechsel keinen signifikanten Einfluss auf die Messergebnisse. Bei der Untersuchung der tatsächlichen Sensorfolienstärke, konnte bei $34 \%$ also 22 Folien eine Dimension von $90 \mu \mathrm{m}$ und bei $65 \%$ also 42 Folien die tatsächliche Dimension von $100 \mu \mathrm{m}$ festgestellt werden. Eine Folie zeigte mit 110 $\mu \mathrm{m}$ eine über der Herstellerangabe liegende Dimensionierung. Die Ergebnisse der vorliegenden Studie lassen sich in Bezug auf die T-Scan Registrierung in engen Zusammenhang mit den seitens Koos et al. (2010) formulierten Erkenntnissen bringen. Somit ist die Annahme zulässig, dass weder der Sensorfolienwechsel, noch die neunmalige Messung mit dem gleichen Sensor oder die Repositionierung des Sensors zwischen den Messungen, einen signifikanten Einfluss auf die Ergebnisse hatten. Ferner wurde betont, dass bei der Anwendung eine hinreichende Routine und Sorgfalt gegeben sein müsse, um reproduzierbare Ergebnisse mit akzeptabler Streubreite zu erhalten (Afrashtehfar und Qadeer 2016; Koos et al. 2010; Tschernitschek et al. 1990).

Unter Berücksichtigung des Studienprotokolls der vorliegenden Untersuchung, wurde auf die reproduzierbare Kopf- und Körperhaltung der Probanden/-innen sowie das genaue Befolgen der definierten Kommandos geachtet. Die Untersuchungen wurden immer von ein und demselben Behandler durchgeführt, wodurch die von Koos et al. und Tschernitschek et al. formulierte Voraussetzung erfüllt war. Die zuvor bemängelte Ungenauigkeit und mangelnde Reproduzierbarkeit der Vorläufergenerationen (Dees et al. 1992; Scholz et al. 1991; Setz und GeisGerstorfer 1990a; Tschernitschek et al. 1990) konnte in der Untersuchung von Koos et al. (2010) mit dem T-Scan III nicht wiedergefunden werden. Bozhkova (2016) untersuchte 30 Studentinnen und Studenten im Alter zwischen 19 und 22 Jahren hinsichtlich ihrer statischen Okklusion und kam zu dem Entschluss, dass die T-Scan-III-Registrierung die einzige reliable Möglichkeit sei, den zeitlichen Ablauf der Okklusion und die relative Kraft der okklusalen Kontakte quantitativ zu ermitteln. Kalachev et al. (2001) untersuchten 15 Studentinnen und Studenten im Alter von 19 bis 24 Jahren anhand von 7-10 Registrierungen je Proband/-in bezüglich der dynamischen Okklusion und kamen zu dem Ergebnis, dass die T-Scan-Registrierung valide und reliable Informationen hinsichtlich der Lokalisation und Verteilung der Kontaktpunkte während der zahngeführten Unterkieferexkursion liefert. 
Cerna et al. (2015) untersuchten 31 zufällig ausgewählte Sensoren an zwei verschiedenen Sensorfolien unter Laborbedingungen, um die Reliabilität und Validität des Messinstrumentes bei gleichbleibender Kraftapplikation zu bestimmen. Sowohl die Reliabilität als auch die Validität in Bezug auf die relative Kraftmessung vielen schwach aus, wohingegen die Ermittlung der applizierten Kraftdauer über alle Sensormessungen sehr valide zu bewerten war. Die Validität und Reliabilität der Kontaktpunktverteilung wurden bei dieser Untersuchung nicht berücksichtig.

Afrashtehfar und Qadeer (2016) wiesen auf die Eigenschaft der Sensorfolie hin, sich währen der Okklusion von $100 \mu \mathrm{m}$ auf $60 \mu \mathrm{m}$ komprimieren zu lassen, was zu Interferenzen im Okklusionsaufbau führen könne. Ferner wurde beschrieben, dass die Sensorfolie zwar auch während der Unterkieferbewegung Interferenzen erzeuge, diese aber ebenso bei konventionellen Okklusionsfolien zu beobachten seien und der T-Scan im Verglich dazu deutlich bessere Auskünfte liefern könne (Afrashtehfar und Qadeer 2016). Unter Berücksichtigung der Studienlage ist augenscheinlich, dass sich der Großteil der Untersuchungen der Reliabilität und Validität des TScan-Verfahrens widmete. In diesem Zusammenhang wird deutlich, wie dringend der Bedarf an zuverlässigen okklusalen Prüfmitteln für die Okklusionsanalyse ist (Kordaß und Lovrov 2013). Die vorliegende Studie befasste sich mit tageszeitabhängigen Abweichungen der statischen und dynamischen Okklusion, in der Annahme diese mit etablierten okklusalen Prüfmitteln belegen zu können. Zieht man in diesem Zusammenhang die Studie von Koos et al. (2010) heran, so wäre auf Basis der Erkenntnisse hinsichtlich der Reliabilität und Validität bei der Verwendung des Systems in vivo von einem präzisen und zuverlässigen Verfahren auszugehen. Signifikante Änderungen in den anhand der T-Scan-III-Registrierung durchgeführten Messungen, könnten damit der Tageszeit bzw. den tageszeitassoziierten Einflüssen auf die Probanden/-innen zugeschrieben werden (Berry und Singh 1983). Andererseits konnte in keinem Verfahren eine signifikante Änderung der Messungen in Bezug auf die im Studiendesign festgelegten Registrierungszeitpunkte gesehen werden. In diesem Zusammenhang ist zu hinterfragen, ob die mangelnde Reliabilität und Validität der untersuchten Messmethoden hierfür verantwortlich waren, oder lediglich in den festgelegten Messzeitpunkten kein Unterschied festzustellen war, was eine Tageszeitabhängigkeit per se nicht ausschließt. Koos et al. (2010) stellten in ihrer Untersuchung einen Messfehler von $1 \%$ und einen nach Bland und Altmann bestimmten 1,96-fachen bzw. 2,77-fachen Messfehler von 2- bzw. 2,8 \% fest (Bland und Altman 1996a, 1996b). Bei dieser statistischen Untersuchung werden die Messgenauigkeit und Variabilität eines Messverfahrens bei jeder Versuchsperson durch mehrfache Messung des Messwertes bestimmt. Die multiplen Messungen werden einem intra- und interindividuellen Vergleich unterzogen und ein Messfehler bestimmt. 
Dieser Messfehler wird dann mit 1,96 und 2,77 multipliziert (Koos et al. 2010). Anschließend kann beurteilt werden, inwieweit der erhobene Messwert vom tatsächlichen Messwert abweicht und so die Messgenauigkeit und Variabilität untersucht werden.

Koos et al. (2010) konnten feststellen, dass $95 \%$ der erhobenen Messungen um weniger als das 1,96-fache vom tatsächlichen Messwert abwichen. Auch bei der Analyse zwischen zwei Wiederholungsmessungen, wichen 95\% der Messungen um weniger als das 2,77-fache vom tatsächlichen Messwert ab, was die Reliabilität und Validität des Verfahrens unterstreiche (Koos et al. 2010).

Unter Berücksichtigung dieser Ergebnisse ist im Zusammenhang mit der vorliegenden Untersuchung zwar von der Verwendung einer hinreichend zuverlässigen Methode auszugehen, jedoch nicht auszuschließen, dass die ermittelte Wahrscheinlichkeit in der Erhebung der statischen Okklusion mittels T-Scan eine Okklusionsänderung pro Zahn von 7,5 \% und pro Kiefer von 20,3\% zu registrieren, letztlich die Restungenauigkeit des Verfahrens abbildete. Diese Annahme kann anhand des Vergleiches der beiden Verfahren zur Erhebung der statischen und dynamischen Okklusion, also der T-Scan-III-Registrierung und der Okklusionsfolien-Registrierung unterstütz werden, da in der vorliegenden Studie sehr konträre Ergebnisse ermittelt wurden. Während die Okklusionsfolienregistrierung in der Erhebung der dynamischen Okklusion nach rechts und links mit einer marginalen Wahrscheinlichkeit von 8,6 bzw. 8,1 \% eine Änderung der Okklusion eines Zahnes messen konnte, war dies im Rahmen der T-Scan-IIIRegistrierung mit einer Wahrscheinlichkeit von 15,2 bzw. 15,1 \% der Fall. Setzt man hierzu die Registrierung der statischen Okklusion in Vergleich, so war die Wahrscheinlichkeit eine Okklusionsänderung eines Zahnes anhand der Okklusionsfolienregistrierung zu erheben, mit $41 \% \mathrm{zu}$ 7,5 \% der T-Scan-III-Registrierung deutlich erhöht. Die Feststellung, dass ein Verfahren zwischen einer statischen und dynamischen Registrierung mit einer geringeren bzw. höheren marginalen Wahrscheinlichkeit Änderungen der Okklusion als das andere Verfahren erkennt, lässt die Zuverlässigkeit der tatsächlichen Abbildung der in vivo Situation hinterfragen. So wäre die TScan-III-Registrierung zur Erhebung der dynamischen Okklusion eventuell besser geeignet, da sie mit deutlich höherer Wahrscheinlichkeit eine Änderung der Okklusion feststellt und die Okklusionsfolienregistrierung für die Erhebung der statischen Okklusion besser geeignet, da sie hierbei mit einer deutlich höheren Wahrscheinlichkeit Änderungen der Okklusion ermittelte. Eine ähnliche nur potenzierte Relation ließ sich bei der Analyse einer Okklusionsänderung pro Kiefer, also von mindestens zwei Zähnen erkennen. Hierbei stellte die Okklusionsfolienregistrierung mit einer marginalen Wahrscheinlichkeit von 98,8 \% eine Okklusionsänderung fest, die T-Scan-III-Registrierung lediglich mit einer Wahrscheinlichkeit von 20,3\%. 
Registrierung der dynamischen Okklusion mittels T-Scan-III konnte hinsichtlich einer Okklusionsänderung pro Kiefer, diese mit einer Wahrscheinlichkeit von 67,7 bzw. 71,7 \% feststellen, wohingegen die Okklusionsfolienregistrierung nur mit einer Wahrscheinlichkeit von 29,6 \%, Änderungen der Okklusion registrierte.

Die Einordnung dieser Ergebnisse ist, besonders unter Berücksichtigung der kontroversen Studienlage (Kordaß und Lovrov 2013), differenziert vorzunehmen. Neben den bereits erläuterten zahlreichen Unterschieden der qualitativen und quantitativen Methoden, lag zwischen der Okklusionsfolienregistrierung und der T-Scan-III-Registrierung vor allem ein enormer Unterschied in der Dimensionierung des Prüfmittels. Obwohl sich die T-ScanSensorfolie, wie durch Afrashtehfar und Qadeer (2016) beschrieben, währen der Okklusion auf $60 \mu \mathrm{m}$ komprimieren ließe, lag sie damit immer noch deutlich über der Dicke der Okklusionsfolie mit $12 \mu \mathrm{m}$. Hinzu kommt, dass sich durch die höhere Materialstärke eine zusätzliche Rigidität des Prüfmittels ergibt. Somit beeinflusste die Sensorfolie die Okklusion nicht nur im Rahmen einer größeren Bisssperrung sondern auch hinsichtlich der Bewegungsfreiheit in der Okklusion, die sich vor allem anhand von Studien mit deutlich dünneren Prüfmitteln wie der Shimstockfolie im Front- und Eckzahnbereich feststellen ließ (Anderson et al. 1993; Woda et al. 1979). Die Vorgängergenerationen des T-Scans wurden vor allem aufgrund ihrer rigiden Sensorfolien kritisiert, die zum Abgleiten der Mandibel während der Registrierung führten (Harvey et al. 1991; Lyons et al. 1992a; Patyk et al. 1989a; Patyk et al. 1989b; Setz und Geis-Gerstorfer 1990b; Walter und Tomomatsu 1990; Yamamura et al. 1990). Koos et al. (2010) stellte zwar mit einer dezidierten Untersuchung fest, dass diese Mängel im TScan III beseitigt seien, dennoch war der Aspekt besonders im Vergleich mit dünneren Prüfmitteln immer noch vorhanden. Eine immer noch gegebene Förderung der Mandibula, abzugleiten, könnte die erhöhte Wahrscheinlichkeit, Änderungen in der dynamischen Okklusion zu registrieren, erklären.

Unter Berücksichtigung der von Williamson und Lundquist (1983) formulierten Zusammenhänge zwischen der Okklusion und den beteiligten Muskelpaaren, könnte durch die größere materialinduzierte Bisssperrung zusätzlich eine unphysiologische Entkopplung der normalerweise führenden Okklusion erzeugt worden sein, die wiederum eine für den/die Probanden/-in atypische Muskelaktivität und damit veränderte dynamische Okklusion bewirkt hat. Über die Auswirkung der Bisssperrung auf die Registrierung wurden bereits die Arbeiten von Halperin et al. (1982) und Forrester et al. (2011) angeführt. 
Halperin et al. (1982) legten hierbei eine Propriozeptionsgrenze für nicht beeinflussende Prüfmittel bis zu einer Stärke von $21 \mu \mathrm{m}$ fest, Forrester et al. (2011) konnten hingegen zeigen, dass trotz stärkerer Prüfmittel wie der $60 \mu \mathrm{m}$ starken Okklusionsseide, ähnliche Muskelaktivitäten mittels Elektromyographie im Vergleich zur Okklusionsfolie $24 \mu \mathrm{m}$ feststellbar waren. Forrester et al. (2011) folgerten aus der geringgradigen Differenz der Muskelaktivitäten bei der Artikulationspapierregistrierung $(202 \mu \mathrm{m})$ und der T-Scan-IIIRegistrierung $(100 \mu \mathrm{m})$, dass vor allem die Steifigkeit des Prüfmittels ausschlaggebend für die Beeinflussung der Registrierung sei.

Unter diesem Aspekt kann abermals die Annahme unterstütz werden, dass die Materialeigenschaft der T-Scan-III-Registrierung einen höheren Einfluss als die Okklusionsfolie auf die Messung hatte. Ferner ist die Annahme zulässig, dass der durch Woda et al. (1979) und Anderson et al. (1993) beschriebene Freiheitsgrad in der maximalen Interkuspidation, durch die verhältnismäßig dicke und steife T-Scan-Sensorfolie während der Messung verlegt wurde, die Probanden/-innen somit immer in die gleiche statische Situation geführt wurden und die okklusale Änderung der Zähne lediglich in der folieninduzierten Auslenkung der Zähne im Parodontalspalt, wie es Millstein (1985) und Murray et al. (1999) bei der Wachsregistrierung beobachteten, begründet lag.

Ein weiterer Aspekt der Okklusionsfolienregistrierung zur Erhebung der dynamischen Okklusion, eine bessere Abbildung der tatsächlichen Situation zuzusprechen, liegt in der Erkenntnis von Plasmans et al. (1988) begründet, deutlich genauere Auskünfte bei Einzelzahnuntersuchungen zu liefern. Da in aller Regel bei der Gesamtheit aller erkannten Führungsmuster an der dynamischen Okklusion weniger Zähne involviert sind, ist anzunehmen, dass die Registrierung in diesem Zusammenhang reliabler als die Erhebung der statischen Okklusion einzustufen ist. Unbedingt muss jedoch auch hierbei die Anfälligkeit gegenüber scharfkantiger Okklusalflächen, wie auch besonders der Speichelbenetzung betont werden (Anderson et al. 1993), der die T-Scan-Registrierung überlegen gegenüber stand. Eine weitere Ursache für höhere Änderungswahrscheinlichkeiten in der T-Scan-Registrierung der dynamischen Okklusion mag ebenfalls in der Dimensionierung begründet sein. So konnten häufiger Balancekontakte festgestellt werden, die sich anhand der Folienregistrierung nicht wiederfinden ließen. Diese Beobachtung ließe sich entweder aufgrund der mangelnden Zeichnungseigenschaft im Bereich der Speichelausführungsgänge erklären, oder aber dadurch, dass die Distanz der Molarenhöcker im Bereich der erkannten Balancekontakte unter $100 \mu \mathrm{m}$ gelegen und die T-Scan-Folie somit falsch positiv registriert hat. Hierbei ist die beeinträchtigte Markierungseigenschaft im posterioren Zahnsegment klar hervorzuheben, dennoch ist ein vergleichsweise steifes, hochdimensioniertes Prüfmittel gerade in sehr knapp-diskludierten 
Zahnrelationen wie im posterioren Zahnsegment bei der Seitwärtsführung des Unterkiefers begünstigt, falsch-positive Ergebnisse zu liefern. Es ist also festzuhalten, dass die Okklusionsfolienregistrierung weniger Interferenzen bei der Seitwärtsbewegung des Unterkiefers auf die Okklusion ausübte und die Anfälligkeit gegenüber feuchten Kontaktoberflächen und die damit verbundene limitierte Zeichnungseigenschaft, vor allem in der Beurteilung aller Zähne in der statischen Okklusion, unbedingt berücksichtigt werden muss.

Die T-Scan-Registrierung übte durch die Materialeigenschaften der Sensorfolie mehr Interferenzen auf die Okklusion aus, lieferte aber einen deutlich höheren Informationsgehalt. Dieser ist jedoch unter Berücksichtigung der Okklusionsbeeinflussung kritisch zu hinterfragen. Hinzu kommt, dass bei allen Prüfverfahren keine quantifizierbare und damit vergleichbare Situation der Muskelaktivität vorausgesetzt werden konnte und somit eine Vergleichbarkeit trotz definierter Kommandos schwierig erscheint. Trotz der vielfach beschriebenen, hohen Reliabilität der T-Scan-III-Registrierung, muss unter Berücksichtigung der Ergebnisse und der bekannten Materialeigenschaften des Verfahrens geschlussfolgert werden, dass sich auch dieses Verfahren nicht als hinreichend präzise erwies, die multifaktoriellen Einflüsse des Tagesverlaufs auf die Okklusion sicher abzubilden.

\subsubsection{Schlussfolgerung}

Nach zusammenhängender Diskussion der Studienergebnisse und vor dem Hintergrund der vorhandenen Literatur kann festgestellt werden, dass sich die Erkenntnisse der vorliegenden Studie im mehrheitlichen wissenschaftlichen Konsens wiederfanden. Es ist hierbei zu betonen, dass die Untersuchung eine möglichst genaue Abbildung der klinischen Behandlungsabläufe gewährleisten sollte, um eine möglichst praxisrelevante Projektion der Erkenntnisse zuzulassen. Es ist hervorzuheben, dass unter Berücksichtigung der Literatur, mehrheitlich die Reliabilität und Validität der Registrierverfahren Gegenstand der Untersuchungen waren. Diese Feststellung scheint besonders vor dem Aspekt nachvollziehbar, dass die Zuverlässigkeit einer Methode grundlegend für sämtliche weitere Untersuchungen ist und erklärt wohlmöglich die schwache Studienlage über die Ermittlung anderer Einflussfaktoren, wie zum Beispiel der Tageszeit.

Da die Literatur vor allem bezüglich neuer quantitativer Verfahren wie beispielsweise der TScan-III-Registrierung ausgesprochen reliable und valide Auskünfte testierte, schien der Versuch, tageszeitabhängige Abweichungen der statischen und dynamischen Okklusion nachzuweisen und diese vergleichend mit herkömmlichen qualitativen Verfahren zu untersuchen lohnend. Die Verfahren der qualitativen Okklusionsanalyse zeigten teils kontroverse Ergebnisse, waren aber besonders vor dem Hintergrund der im Rahmen anderer Studien beschriebenen Schwächen der einzelnen Prüfmittel aufgrund mangelnder Reliabilität und Validität nicht geeignet, 
eine tageszeitassoziierte Änderung der Okklusion sicher zu belegen. Obwohl in nahezu allen Verfahren signifikant andere Ergebnisse erkennbar waren, kann aufgrund des nicht vorhandenen Goldstandards im Sinne einer absolut präzisen Analyse der tatsächlichen Okklusion der Patientinnen und Patienten, keine Aussage darüber getroffen werden, ob die Änderungen auf Messfehlern oder tatsächlich der Tageszeit beruhten.

Darüber hinaus ist zu betonen, dass in der vorliegenden Studie keine quantitative Ermittlung der tatsächlich applizierten Kaukraft im Sinne einer elektromyographischen Überwachung während der Registrierung durchgeführt wurde, was bei einer Vergleichsanalyse nach gegenwärtigem Kenntnisstand statthaft gewesen wäre, da die beträchtliche Einflussnahme auf die Okklusion in diesem Zusammenhang mehrheitlich konsentiert beschrieben wurde. Zusätzlich ist hervorzuheben, dass ebenfalls keine quantitative Auswertung im Sinne der computergestützten Transillumination der Wachs- und Silikonregistrierung angewandt wurde, die eventuell - vor allem im Falle der Silikonregistrierung - zu deutlich verlässlicheren Auskünften hätte führen können. Hinsichtlich des quantitativen Verfahrens T-Scan III, ist vor allem die Beeinflussung der Okklusion im Rahmen der Messung kritisch zu bewerten, obwohl das Verfahren im Rahmen zahlreicher Untersuchungen als ausreichend reliabel eingestuft wurde. Ähnlich der angeführten Problematik qualitativer Messmethoden, ist auch bei der T-Scan-III-Registrierung von einem geringen aber immer noch gegebenen Messfehler auszugehen, der eine klare Einordnung der Ergebnisse in Abhängigkeit zu tageszeitassoziierten Einflüssen nicht zulässt. Eine adjuvante elektromyographische Überwachung hätte zwar zur Reliabilität der erhobenen Messungen beigetragen, eine klare Bestätigung des Untersuchungsfokus jedoch ebenfalls nicht zugelassen. Sofern diesem Verfahren eine definitive Messgenauigkeit zugesprochen werden könnte, wäre letztlich immer noch die Abbildung der tatsächlichen Situation der Patientinnen und Patienten zu hinterfragen, da das Messinstrument eine zu deutliche Einflussnahme auf die Okklusion ausübte.

Es konnte festgestellt werden, dass die durchgeführte Studie mit dem Ziel tageszeitabhängige Abweichungen der Okklusion festzustellen eine ausgesprochen hohe Präzision verlangte. Keine der untersuchten Registrierungsmethoden konnte unter praxisüblicher Anwendung der Materialien diesem Bedarf hinreichend gerecht werden. Nach intensiver Beleuchtung der individuellen Vor- und Nachteile der untersuchten Okklusionsanalysen ist der Silikonregistrierung eine im Vergleich hohe Aussagekraft zu testieren, da es über die am besten geeigneten Materialeigenschaften zur klinischen Okklusionsanalyse verfügte. Trotz der, aus bereits formulierten Gründen, nicht durchgeführten Quantifizierung bzw. Befundung der Bisskraft und der dadurch nicht gegeben Reliabilität der Methode, konnte das Verfahren glaubhaft auf okklusale Änderungen der statischen Okklusion hinweisen, die es in Zukunft in einem optimierten Versuchsaufbau zu 
belegen gilt. Vor allem unter dem in zahlreichen Studien betonten Aspekt der prüfmittelinduzierten Beeinflussung der Okklusion, ist in Zukunft die Kapazität volldigitaler Okklusionsscanner hinsichtlich ihrer Reliabilität und Validität zu prüfen, da sie außerhalb des zu untersuchenden Kontaktpunktareals registrieren. Die Beeinflussung der Okklusion durch variable Muskelaktivitäten muss jedoch auch hierbei Berücksichtigung finden, um sich der Zielsetzung der vollkommenen Reliabilität, sofern diese überhaupt realisierbar ist, anzunähern. 


\section{Zusammenfassung}

Die vorliegende Studie untersuchte tageszeitabhängige Abweichungen der statischen und dynamischen Okklusion anhand neun verschiedener Registrierverfahren zu drei Tageszeiten an je zwei Untersuchungstagen in 14-tägigem Abstand. Zusätzlich wurden die Ergebnisse der einzelnen Verfahren ausgewertet und in vergleichenden Zusammenhang gesetzt. Alle verwendeten okklusalen Prüfmittel sind den gängigen Verfahren zur Okklusionsüberprüfung zuzuordnen und in ihrer klinischen Verwendung erprobt und angesehen. Zielsetzung der vorliegenden Studie war es, die Variabilität des menschlichen Bisses zu untersuchen und darüber hinaus die etablierten Verfahren zur Okklusionsanalyse auf Ihre Aussagekraft in diesem Zusammenhang zu untersuchen.

Die Auswertung der Tageszeitabhängigkeit ergab zwar in Bezug auf die ausgewählten Messzeitpunkte keine signifikanten Unterschiede, dennoch konnte in vier von neun Verfahren mit einer Wahrscheinlichkeit von mehr als 50 \% eine Änderung der Okklusionsmessung pro Kiefer registriert werden. Die Wahrscheinlichkeit in aufeinanderfolgenden Messungen einer beliebigen Tageszeit Okklusionsänderungen zur registrieren, war somit höher als keine Änderungen zu sehen. Dieser Beobachtung ist eine klinische Relevanz beizumessen, da in der zahnärztlich-prothetischen, wie auch -konservierenden Patientenversorgung in aller Regel von einer konstanten Okklusion ausgegangen wird. Hieraus resultiert sowohl aus Sicht der Patienten als auch der Behandelnden eine entsprechende Erwartungshaltung, die selten auf Anhieb erfüllt werden kann, da Nachjustierungen vorgenommen werden müssen. Die Beobachtung von inkonstanten okklusalen Verhältnissen sollte daher bei einer umfassenden Patientenaufklärung berücksichtigt werden, um darüber hinaus auch für die Herausforderung der zahnärztlichen Versorgung zu sensibilisieren.

Um dieser Herausforderung angemessen zu begegnen, hält die zahnärztliche Behandlung eine Vielzahl okklusaler Prüfmittel vor, deren gängigste Vertreter in dieser Studie ebenfalls untersucht wurden. Es konnte festgestellt werden, dass nicht alle Registrierverfahren gleichermaßen für sämtliche Anforderungsbereiche der zahnärztlichen Behandlung geeignet sind. Somit sollte das okklusale Prüfmittel immer anwendungsbezogen ausgewählt bzw. durch andere Verfahren ergänzt werden. Von der Formulierung eines Goldstandards für die zahnärztliche Okklusionsregistrierung sollte daher abgesehen werden, da alle untersuchten Prüfmittel neben ihren geschätzten Eigenschaften auch Mängel aufwiesen. Besonders sei hierbei auf die Affinität der Okklusionsfolie $12 \mu \mathrm{m}$ hingewiesen, mit überdurchschnittlich hoher Wahrscheinlichkeit Okklusionsveränderungen zu erfassen. 
Ihr gegenüber standen die Verfahren der Wachs- und Shimstockregistrierung, die mit einer Wahrscheinlichkeit von 7,4 bzw. 13,8 \% nur selten Okklusionsveränderungen registrieren konnten. Die Silikon- wie auch T-Scan-Registrierung der statischen Okklusion bildeten, mit einer Wahrscheinlichkeit von 66,3 bzw. 20,3\% eine Okklusionsänderung pro Kiefer anzuzeigen, das Mittelfeld ab. Umgekehrt verhielt es sich mit der Erfassung der dynamischen Okklusion, wobei anhand der Folienregistrierung geringere Änderungswahrscheinlichkeiten als bei der digitalen T-Scan-Analyse festgestellt werden konnten. Die Zusammenfassung aller dargestellten Erkenntnisse fordert eine sorgfältige Auswahl bzw. Kombination der okklusalen Prüfmittel unter besonderer Berücksichtigung der inkonstanten individuellen Patientenokklusion. 


\section{Anhang}

\subsection{Deskriptive Werte der erhobenen Daten}

Tabelle A1: Deskriptive Werte

\begin{tabular}{|c|c|c|}
\hline Parameter & Niveau & Wert \\
\hline $\mathrm{n}$ & & 1608 \\
\hline \multicolumn{3}{|l|}{ Zahn $[11 / . . / 28]$} \\
\hline & 11 & $114(7,1 \%)$ \\
\hline & 12 & $114(7,1 \%)$ \\
\hline & 13 & $114(7,1 \%)$ \\
\hline & 14 & $114(7,1 \%)$ \\
\hline & 15 & $102(6,3 \%)$ \\
\hline & 16 & $114(7,1 \%)$ \\
\hline & 17 & $114(7,1 \%)$ \\
\hline & 18 & $18(1,1 \%)$ \\
\hline & 21 & $114(7,1 \%)$ \\
\hline & 22 & $114(7,1 \%)$ \\
\hline & 23 & $114(7,1 \%)$ \\
\hline & 24 & $114(7,1 \%)$ \\
\hline & 25 & $102(6,3 \%)$ \\
\hline & 26 & $114(7,1 \%)$ \\
\hline & 27 & $114(7,1 \%)$ \\
\hline & 28 & $18(1,1 \%)$ \\
\hline \multicolumn{3}{|l|}{ Seite } \\
\hline & links & $804(50,0 \%)$ \\
\hline & rechts & $804(50,0 \%)$ \\
\hline
\end{tabular}




\begin{tabular}{|c|c|c|}
\hline Parameter & Niveau & Wert \\
\hline \multicolumn{3}{|l|}{ Wachs } \\
\hline & Mittelwert \pm SD & $4,1 \pm 2,9$ \\
\hline & Median (min; max) & $4(0 ; 17)$ \\
\hline \multicolumn{3}{|l|}{ Folie dyn li } \\
\hline & Mittelwert \pm SD & $0,34 \pm 0,51$ \\
\hline & Median (min; max) & $0(0 ; 4)$ \\
\hline \multicolumn{3}{|l|}{ Folie dyn re } \\
\hline & Mittelwert \pm SD & $0,38 \pm 0,54$ \\
\hline & Median (min; max) & $0(0 ; 3)$ \\
\hline \multicolumn{3}{|l|}{ Folie stat } \\
\hline & Mittelwert \pm SD & $3,7 \pm 2,7$ \\
\hline & Median (min; max) & $4(0 ; 11)$ \\
\hline \multicolumn{3}{|l|}{ Registrado } \\
\hline & Mittelwert \pm SD & $4,5 \pm 3,2$ \\
\hline & Median (min; max) & $4(0 ; 17)$ \\
\hline \multicolumn{3}{|l|}{ Shimstock } \\
\hline & Minus & $128(8,0 \%)$ \\
\hline & PlusMinus & $118(7,3 \%)$ \\
\hline & Plus & $1362(84,7 \%)$ \\
\hline \multicolumn{3}{|l|}{ T-Scan dyn li } \\
\hline & Mittelwert \pm SD & $7 \pm 20$ \\
\hline & Median (min; max) & $0(0 ; 100)$ \\
\hline \multicolumn{3}{|l|}{ T-Scan dyn re } \\
\hline & Mittelwert \pm SD & $7 \pm 20$ \\
\hline & Median (min; max) & $0(0 ; 100)$ \\
\hline
\end{tabular}




\begin{tabular}{|c|c|c|}
\hline Parameter & Niveau & Wert \\
\hline T-Scan stat & & $6,8 \pm 5$ \\
\hline & Mittelwert \pm SD & $5,9(0 ; 38)$ \\
\hline
\end{tabular}

Absolute und relative Häufigkeiten für kategorielle Variablen; Mittelwert \pm SD und Median (min;max) für kontinuierliche Variablen; Median(min;max) für ordinal skalierte Variablen.

\subsection{Logistische Regression}

Tabelle A2: Logistische Regression

\begin{tabular}{|c|c|c|c|c|c|c|c|}
\hline $\begin{array}{l}\text { Untersu- } \\
\text { chung }\end{array}$ & Niveau & U.-2 & N.-2 & $\begin{array}{c}\text { Schätz- } \\
\text { wert }\end{array}$ & $\begin{array}{l}\text { Kon- } \\
\text { fidenzin- } \\
\text { tervall }\end{array}$ & p-Wert & $\begin{array}{l}\text { Zei- } \\
\text { chen }\end{array}$ \\
\hline & & & & 0,019 & $\begin{array}{c}{[0,011 ;} \\
0,032]\end{array}$ & $<0,001$ & $*$ \\
\hline Seite & links & & & & & & \\
\hline Seite & rechts & & & 0,888 & $\begin{array}{c}{[0,783} \\
1,007]\end{array}$ & 0,063 & . \\
\hline time & 12 & & & & & & \\
\hline time & 16 & & & 1,221 & $\begin{array}{c}{[0,656} \\
2,272]\end{array}$ & 0,529 & \\
\hline Verfahren & Wachs & & & & & & \\
\hline Verfahren & Folie dyn li & & & 2,843 & $\begin{array}{c}{[1,649 ;} \\
4,902]\end{array}$ & $<0,001$ & $*$ \\
\hline Verfahren & Folie dyn re & & & 3,037 & $\begin{array}{c}{[1,768} \\
5,217]\end{array}$ & $<0,001$ & $*$ \\
\hline Verfahren & Folie stat & & & 20,350 & $\begin{array}{l}{[12,435} \\
33,304]\end{array}$ & $<0,001$ & $*$ \\
\hline Verfahren & Registrado & & & 6,594 & $\begin{array}{l}{[3,963 ;} \\
10,970]\end{array}$ & $<0,001$ & $*$ \\
\hline
\end{tabular}




\begin{tabular}{|c|c|c|c|c|c|c|c|}
\hline $\begin{array}{l}\text { Untersu- } \\
\text { chung }\end{array}$ & Niveau & U.-2 & N.-2 & $\begin{array}{c}\text { Schätz- } \\
\text { wert }\end{array}$ & $\begin{array}{c}\text { Kon- } \\
\text { fidenzin- } \\
\text { tervall }\end{array}$ & p-Wert & $\begin{array}{l}\text { Zei- } \\
\text { chen }\end{array}$ \\
\hline Verfahren & Shimstock & & & 0,999 & $\begin{array}{c}{[0,522} \\
1,911]\end{array}$ & 0,997 & \\
\hline Verfahren & T-Scan dyn li & & & 4,485 & $\begin{array}{c}{[2,661} \\
7,557]\end{array}$ & $<0,001$ & $*$ \\
\hline Verfahren & $\begin{array}{c}\text { T-Scan dyn } \\
\text { re }\end{array}$ & & & 5,698 & $\begin{array}{c}{[3,410} \\
9,520]\end{array}$ & $<0,001$ & $*$ \\
\hline Verfahren & T-Scan stat & & & 2,340 & $\begin{array}{c}{[1,339 ;} \\
4,088]\end{array}$ & 0,003 & $*$ \\
\hline Zahns & 1 & & & & & & \\
\hline Zahns & 2 & & & 1,175 & $\begin{array}{c}{[0,889} \\
1,553]\end{array}$ & 0,256 & \\
\hline Zahns & 3 & & & 1,131 & $\begin{array}{c}{[0,854} \\
1,497]\end{array}$ & 0,391 & \\
\hline Zahns & 4 & & & 2,306 & $\begin{array}{c}{[1,786} \\
2,979]\end{array}$ & $<0,001$ & * \\
\hline Zahns & 5 & & & 2,164 & $\begin{array}{c}{[1,660} \\
2,820]\end{array}$ & $<0,001$ & * \\
\hline Zahns & 6 & & & 2,858 & $\begin{array}{c}{[2,224} \\
3,674]\end{array}$ & $<0,001$ & $*$ \\
\hline Zahns & 7 & & & 2,924 & $\begin{array}{c}{[2,276} \\
3,756]\end{array}$ & $<0,001$ & $*$ \\
\hline Zahns & 8 & & & 3,640 & $\begin{array}{c}{[2,315} \\
5,722]\end{array}$ & $<0,001$ & $*$ \\
\hline Verfahren & Wachs & $*$ & $*$ & & & & \\
\hline Verfahren & Folie dyn li & time & 12 & & & & \\
\hline Verfahren & Folie dyn li & time & 16 & 0,621 & $\begin{array}{c}{[0,290} \\
1,331]\end{array}$ & 0,221 & \\
\hline
\end{tabular}




\begin{tabular}{|c|c|c|c|c|c|c|c|}
\hline $\begin{array}{l}\text { Untersu- } \\
\text { chung }\end{array}$ & Niveau & U.-2 & N.-2 & $\begin{array}{c}\text { Schätz- } \\
\text { wert }\end{array}$ & $\begin{array}{l}\text { Kon- } \\
\text { fidenzin- } \\
\text { tervall }\end{array}$ & p-Wert & $\begin{array}{l}\text { Zei- } \\
\text { chen }\end{array}$ \\
\hline Verfahren & Folie dyn re & time & 12 & & & & \\
\hline Verfahren & Folie dyn re & time & 16 & 0,631 & $\begin{array}{c}{[0,297 ;} \\
1,342]\end{array}$ & 0,232 & \\
\hline Verfahren & Folie stat & time & 12 & & & & \\
\hline Verfahren & Folie stat & time & 16 & 0,812 & $\begin{array}{c}{[0,416} \\
1,588]\end{array}$ & 0,544 & \\
\hline Verfahren & Registrado & time & 12 & & & & \\
\hline Verfahren & Registrado & time & 16 & 0,768 & $\begin{array}{c}{[0,383} \\
1,541]\end{array}$ & 0,457 & \\
\hline Verfahren & Shimstock & time & 12 & & & & \\
\hline Verfahren & Shimstock & time & 16 & 0,729 & $\begin{array}{c}{[0,293} \\
1,815]\end{array}$ & 0,497 & \\
\hline Verfahren & T-Scan dyn li & time & 12 & & & & \\
\hline Verfahren & T-Scan dyn li & time & 16 & 1,026 & $\begin{array}{c}{[0,505} \\
2,084]\end{array}$ & 0,943 & \\
\hline Verfahren & $\begin{array}{c}\text { T-Scan dyn } \\
\text { re }\end{array}$ & time & 12 & & & & \\
\hline Verfahren & $\begin{array}{c}\text { T-Scan dyn } \\
\text { re }\end{array}$ & time & 16 & 0,645 & $\begin{array}{c}{[0,318 ;} \\
1,309]\end{array}$ & 0,224 & \\
\hline Verfahren & T-Scan stat & time & 12 & & & & \\
\hline Verfahren & T-Scan stat & time & 16 & 0,798 & $\begin{array}{c}{[0,370} \\
1,719]\end{array}$ & 0,564 & \\
\hline
\end{tabular}

In den Spalten Schätzwert und Konfidenzintervall sind die Oddsratios mit dem 95\% Konfidenzintervall angegeben. Die Spalte p-Wert zeigt den zugehörigen p-Wert. In der letzten Spalte sind alle Koeffizienten mit einem $\mathrm{p}-\mathrm{W}$ ert $<0,05$ mit einem Stern markiert. 


\subsection{Logistische Regression ohne Tageszeitinteraktion}

Tabelle A3: Logistische Regression ohne Interaktion mit der Tageszeit

\begin{tabular}{|c|c|c|c|c|c|}
\hline $\begin{array}{l}\text { Untersu- } \\
\text { chung }\end{array}$ & Niveau & Schätzwert & $\begin{array}{l}\text { Konfidenzinter- } \\
\text { vall }\end{array}$ & p-Wert & $\begin{array}{l}\text { Zei- } \\
\text { chen }\end{array}$ \\
\hline & & 0,022 & {$[0,015 ; 0,033]$} & $<0,001$ & $*$ \\
\hline Seite & links & & & & \\
\hline Seite & rechts & 0,888 & {$[0,783 ; 1,007]$} & 0,063 & . \\
\hline time & 12 & & & & \\
\hline time & 16 & 0,944 & {$[0,833 ; 1,070]$} & 0,370 & \\
\hline Verfahren & Wachs & & & & \\
\hline Verfahren & Folie dyn li & 2,249 & {$[1,539 ; 3,286]$} & $<0,001$ & $*$ \\
\hline Verfahren & Folie dyn re & 2,419 & {$[1,661 ; 3,522]$} & $<0,001$ & $*$ \\
\hline Verfahren & Folie stat & 18,269 & {$[13,064 ; 25,549]$} & $<0,001$ & * \\
\hline Verfahren & Registrado & 5,756 & {$[4,065 ; 8,149]$} & $<0,001$ & * \\
\hline Verfahren & Shimstock & 0,851 & {$[0,540 ; 1,341]$} & 0,486 & \\
\hline Verfahren & T-Scan dyn li & 4,543 & {$[3,191 ; 6,467]$} & $<0,001$ & $*$ \\
\hline Verfahren & T-Scan dyn re & 4,577 & {$[3,216 ; 6,515]$} & $<0,001$ & $*$ \\
\hline Verfahren & T-Scan stat & 2,081 & {$[1,418 ; 3,053]$} & $<0,001$ & $*$ \\
\hline Zahns & 1 & & & & \\
\hline Zahns & 2 & 1,176 & {$[0,890 ; 1,554]$} & 0,255 & \\
\hline Zahns & 3 & 1,131 & {$[0,854 ; 1,497]$} & 0,390 & \\
\hline Zahns & 4 & 2,306 & {$[1,786 ; 2,978]$} & $<0,001$ & * \\
\hline Zahns & 5 & 2,164 & {$[1,660 ; 2,820]$} & $<0,001$ & $*$ \\
\hline Zahns & 6 & 2,857 & {$[2,223 ; 3,672]$} & $<0,001$ & $*$ \\
\hline Zahns & 7 & 2,922 & {$[2,275 ; 3,754]$} & $<0,001$ & $*$ \\
\hline
\end{tabular}




\begin{tabular}{|c|c|c|c|c|c|}
\hline $\begin{array}{c}\text { Untersu- } \\
\text { chung }\end{array}$ & Niveau & Schätzwert & $\begin{array}{c}\text { Konfidenzinter- } \\
\text { vall }\end{array}$ & p-Wert & $\begin{array}{c}\text { Zei- } \\
\text { chen }\end{array}$ \\
\hline Zahns & 8 & 3,638 & {$[2,314 ; 5,719]$} & $<0,001$ & $*$ \\
\hline
\end{tabular}

In den Spalten Schätzwert und Konfidenzintervall sind die Oddsratios mit dem 95\% Konfidenzintervall angegeben. Die Spalte p-Wert zeigt den zugehörigen p-Wert. In der letzten Spalte sind alle Koeffizienten mit einem $\mathrm{p}-\mathrm{W}$ ert $<0,05$ mit einem Stern markiert.

\subsection{Logistische Regression zur Okklusionsänderung pro Kiefer}

Tabelle A4: Logistische Regression zur Okklusionsänderung pro Kiefer

\begin{tabular}{|c|c|c|c|c|c|}
\hline $\begin{array}{c}\text { Untersu- } \\
\text { chung }\end{array}$ & Niveau & $\begin{array}{c}\text { Schätz- } \\
\text { wert }\end{array}$ & Konfidenzintervall & p-Wert & $\begin{array}{c}\text { Zei- } \\
\text { chen }\end{array}$ \\
\hline time & 12 & 0,091 & {$[0,038 ; 0,218]$} & $<0,001$ & $*$ \\
\hline time & 16 & 0,784 & {$[0,537 ; 1,147]$} & 0,210 & \\
\hline Verfahren & Wachs & & & & \\
\hline Verfahren & Folie dyn li & 5,236 & {$[1,975 ; 13,878]$} & 0,001 & $*$ \\
\hline Verfahren & Folie dyn re & 5,236 & {$[1,975 ; 13,879]$} & 0,001 & $*$ \\
\hline Verfahren & Folie stat & 1006,064 & {$[116,688 ; 8674,096]$} & $<0,001$ & $*$ \\
\hline Verfahren & Registrado & 24,498 & {$[9,263 ; 64,789]$} & $<0,001$ & $*$ \\
\hline Verfahren & Shimstock & 1,994 & {$[0,693 ; 5,738]$} & 0,201 & \\
\hline Verfahren & T-Scan dyn li & 31,525 & {$[11,777 ; 84,384]$} & $<0,001$ & $*$ \\
\hline Verfahren & T-Scan dyn re & 26,039 & {$[9,821 ; 69,040]$} & $<0,001$ & $*$ \\
\hline Verfahren & T-Scan stat & 3,173 & {$[1,159 ; 8,683]$} & 0,025 & $*$ \\
\hline
\end{tabular}

Ein Veränderungsevent pro Kiefer (mindestens zwei Zähne mit einer Änderung in der Okklusionsmessung) wurde in Abhängigkeit von der Tageszeit (12 Uhr gegen 9 Uhr und 16 Uhr gegen 9 Uhr) und vom Messverfahren modelliert. In den Spalten Schätzwert und Konfidenzintervall sind die Oddsratios mit dem 95\% Konfidenzintervall angegeben. Die Spalte p-Wert zeigt den zugehörigen p-Wert. In der letzten Spalte sind alle Koeffizienten mit einem p-Wert $<0,05$ mit einem Stern markiert. 


\section{$7 \quad$ Literaturverzeichnis}

Abduo J, Tennant M, McGeachie J (2013): Lateral occlusion schemes in natural and minimally restored permanent dentition: a systematic review. J Oral Rehabil $\underline{40}, 788-802$

Afrashtehfar KI, Qadeer S (2016): Computerized occlusal analysis as an alternative occlusal indicator. Cranio $\underline{34}, 52-57$

Ahlers MO, Biffar R, Freesmeyer W, Hugger A, Koeck B, Kordaß B, Meyer G, Ottl P, Seeher WD, Fuchs WB et al. (2006): Terminologieliste der Deutschen Gesellschaft für Funktionsdiagnostik und Therapie (DGFDT) und der Deutschen Gesellschaft für zahnärztliche Prothetik und Werkstoffkunde (DGzPW). Dtsch Zahnarztl Z $\underline{61}, 1-7$

Ahlers MO, Jakstat H (2015): CMD-Screening mit dem „CMD-Kurzbefund“. ZWR $\underline{124}$, 102106

Ai M, Ishiwara T (1968): A study of masticatory movementat the incision inferius. Bull Tokyo Med Dent Univ 15, 371-386

Alexander SA (1996): Levels of root resorption associated with continuous arch and sectional arch mechanics. Am J Orthod Dentofacial Orthop 110, 321-324

Al-Hiyasat AS, Abu-Alhaija ES (2004): The relationship between static and dynamic occlusion in 14-17-year-old school children. J Oral Rehabil 31, 628-633

Alkan A, Arici S, Sato S (2006): Bite force and occlusal contact area changes following mandibular widening using distraction osteogenesis. Oral Surg Oral Med Oral Pathol Oral Radiol En$\operatorname{dod} \underline{101}, 432-436$

Alkan A, Bulut E, Arici S, Sato S (2008): Evaluation of treatments in patients with nocturnal bruxism on bite force and occlusal contact area. Eur J Dent 2, 276-282

Alt KW, Kullmer O, Türp JC (2017): Okklusion - Kultur versus Natur. Zahnarztl Mitt 10, 5864

Anderson DJ, Picton DC (1957): Tooth contact during chewing. J Dent Res $\underline{36}$, 21-26

Anderson GC, Schulte JK, Aeppli DM (1993): Reliability of the evaluation of occlusal contacts in the intercuspal position. J Prosthet Dent $\underline{70}, 320-323$

Andrews LF (1972): The six keys to normal occlusion. Am J Orthod 62, 296-309

Angel JL (1948): Factors in temporomandibular joint form. Am J Anat 모, 223-246

Angle EH: The angle system of regulation and retention of the teeth, 1. Auflage; S.S. White Dental Manufacturing Philadelphia 1887

Anup G, Ahila SC, Vasanthakumar M (2011): Evaluation of dimensional stability, accuracy and surface hardness of interocclusal recording materials at various time intervals: an in vitro study. J Indian Prosthodont Soc 11, 26-31

Aras K, Hasanreisoğlu U, Shinogaya T (2009): Masticatory performance, maximum occlusal force, and occlusal contact area in patients with bilaterally missing molars and distal extension removable partial dentures. Int J Prosthodont 22, 204-209 
Aslam K, Nidam R, Ali Syed S (2018): Dependence of canine-guided and group guided occlusion on Angle's classification and gender. J Pak Dent Assoc 27, 67-70

Baba K, Tsukiyama Y, Clark GT (2000): Reliability, validity, and utility of various occlusal measurement methods and techniques. J Prosthet Dent $\underline{83}$, 83-89

Baldini A, Nota A, Tripodi D, Longoni S, Cozza P (2013): Evaluation of the correlation between dental occlusion and posture using a force platform. Clinics (Sao Paulo) $\underline{68}$, 45-49

Bates D, Mächler M, Bolker B, Walker S (2015): Fitting linear mixed-effects models using lme4. J Stat Softw $\underline{67}, 1-51$

Becker CM, Kaiser DA (1993): Evolution of occlusion and occlusal instruments. J Prosthodont $\underline{2}, 33-43$

Belser UC, Hannam AG (1985): The influence of altered working-side occlusal guidance on masticatory muscles and related jaw movement. J Prosthet Dent $\underline{53}$, 406-413

Bernhardt O, Meyer G, Schülein H (2017): Anterior/canine guidance - prevalence, clinical parameters, and value for prosthetic restorations. An update for clinical practice. J CranioMand Func $2,123-133$

Berry DC, Singh BP (1983): Daily variations in occlusal contacts. J Prosthet Dent $\underline{50}$, 386-391

Berry DC, Wilmot G (1977): The use of a biofeedback technique in the treatment of mandibular dysfunction pain. A preliminary report on the Myotron 220. J Oral Rehabil 4, 255-260

Beyron H (1954): Characteristics of functionally optimal occlusion and principles of occlusal rehabilitation. J Am Dent Assoc $\underline{6}$, 648-656

Beyron H (1964): Occlusal relations and mastication in australian aboriginies. Acta Odontol Scand 22, 597-678

Beyron H (1969): Optimal occlusion. Dent Clin North Am 13, 537-554

Bland JM, Altman DG (1996a): Measurement error. Br Med J 312, 1654

Bland JM, Altman DG (1996b): Measurement error. Br Med J $\underline{313}, 744$

Boening KW, Walter MH (1992): Computer-aided evaluation of occlusal load in complete dentures. J Prosthet Dent 67, 339-344

Böhm A, Rammelsberg P, Pho Duc JM, May HC, Pospiech P, Gernet W (1995a): Direkte dreidimensionale elektronische Kondylenpositionsanalysen zur Bestimmung von RKP-IKPDiskrepanzen. Dtsch Zahnarztl Z 7, 35-39

Böhm A, Rammelsberg P, Pho Duc JM, May HC, Pospiech P, Gernet W (1995b): Elektronische Kondylenpositionsanalyse bei Gesunden und Patienten mit anteriorer Diskusverlagerung im Kiefergelenk. Dtsch Zahnarztl Z 7, 553-557

Bozhkova TP (2016): The T-SCAN System in Evaluating Occlusal Contacts. Folia Med $\underline{58}$, 122-130 
Carey JP, Craig M, Kerstein RB, Radke J (2007): Determining a relationship between applied occlusal load and articulating paper mark area. Open Dent J $1,1-7$

Cerna M, Ferreira R, Zaror C, Navarro P, Sandoval P (2015): In vitro evaluation of T-Scan ${ }^{\circledR I I I}$ through study of the sensels. Cranio $\underline{33}, 299-305$

Christensen LV (1981): Jaw muscle fatigue and pains induced by experimental tooth clenching: a review. J Oral Rehabil $\underline{8}, 27-36$

Ciancaglini R, Gherlone EF, Radaelli G (2003): Unilateral temporomandibular disorder and

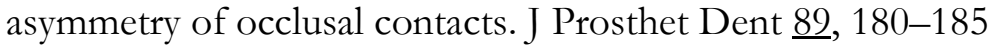

Ciancaglini R, Gherlone EF, Redaelli S, Radaelli G (2002): The distribution of occlusal contacts in the intercuspal position and temporomandibular disorder. J Oral Rehabil $\underline{29}, 1082-1090$

Clark JR, Evans RD (2001): Functional occlusion. J Orthod 28, 76-81

Coelho MF, Cavalcanti Bd, Claro Neves AC, Jóias RP, Rode Sd (2015): Influence of dental chair backrest inclination on the registration of the mandibular position. J Prosthet Dent 114 , 693695

D'Amico A (1961): Functional occlusion of the natural teeth of man. J Prosthet Dent 11 , 899_ 915

Davies S, Al-Ani Z, Jeremiah H, Winston D, Smith P (2005): Reliability of recording static and dynamic occlusal contact marks using transparent acetate sheet. J Prosthet Dent $\underline{94}, 458-461$

Dees A, Kess K, Proff P (1992): Der Einsatz des T-Scan-Systems in der okklusalen Diagnostik. Dtsch Zahn Mund Kieferheilkd Zentralbl, 145-151

DeLong R, Knorr S, Anderson GC, Hodges J, Pintado MR (2007): Accuracy of contacts calculated from 3D images of occlusal surfaces. J Dent $\underline{35}, 528-534$

Dinçer M, Meral O, Tümer N (2003): The investigation of occlusal contacts during the retention period. Angle Orthod 73, 640-646

Durbin DS, Sadowsky C (1986): Changes in tooth contacts following orthodontic treatment. Am J Orthod Dentofacial Orthop 무, 375-382

Ehrlich J, Taicher S (1981): Intercuspal contacts of the natural dentition in centric occlusion. J Prosthet Dent $\underline{45}$, 419-421

Engelhardt JP, Hugger A, Jakstat H, Kordaß B (2002): Instrumentelle Funktionsanalyse - Prinzipien und Anwendung. Dtsch Zahnarztl Z

Ferrario VF, Serrao G, Dellavia C, Caruso E, Sforza C (2002): Relationship between the number of occlusal contacts and masticatory muscle activity in healthy young adults. Cranio $\underline{20}$, 91-98

Forrester SE, Presswood RG, Toy AC, Pain MT (2011): Occlusal measurement method can affect SEMG activity during occlusion. J Oral Rehabil $\underline{38}$, 655-660

Francová K, Eber M, Zapletalová J (2015): Functional occlusal patterns during lateral excursions in young adults. J Prosthet Dent $\underline{113}, 571-577$ 
Freesmeyer WB (1991): Elektronische Okklusionsdiagnostik mit dem T-Scanner, Möglichkeiten und Grenzen. Quintessenz J 9, 1443-1454

Friel S (1954): The development of ideal occlusion of the gum pads and teeth. Am J Orthod $\underline{40}$, $196-227$

Frömder B (1989): Untersuchungen über Zusammenhänge zwischen der Zahnführung bei Laterotrusion und der Bisslage. Dtsch Zahnarztl Z $\underline{44}, 70-72$

Fukushima S (2016): A controversy with respect to occlusion. Jpn Dent Sci Rev $\underline{52}$, 49-53

Garg AK (2007): Analyzing dental occlusion for implants. Dent Implantol Update 18, 65-70

Garrido Garcia VC, Garcia Cartagena A, Gonzalez Sequeros O (1997): Evaluation of occlusal contacts in maximum intercuspation using the T-Scan system. J Oral Rehabil 24, 899-903

Gauder M: Computerunterstützte Okklusionsanalyse zur Symmetrie der okklusalen Kontaktpunktverteilung. Med. Diss. Düsseldorf 2010

Gazit E, Fitzig S, Lieberman MA (1986): Reproducibility of occlusal marking techniques. J Prosthet Dent $\underline{55}, 505-509$

Ghazal M, Ludwig K, Kern M (2008): Evaluation of vertical accuracy of interocclusal recording materials. Quintessenz J 39, 727-732

Gillings BRD, Graham CH, Duckmanton NA (1973): Jaw movements' in young adult men during chewing. J Prosthet Dent 29, 616-627

González Sequeros O, Garrido García VC, García Cartagena A (1997): Study of occlusal contact variability within individuals in a position of maximum intercuspation using the T-SCAN system. J Oral Rehabil 24, 287-290

Graber TM, Vanarsdall RL: Kieferorthopädie; Urban und Fischer München 1999

Graf H, Zander HA (1963): Tooth contact patterns in mastication. J Prosthet Dent $\underline{13}$, 10551066

Gregory WK, Broadbent BH, Hellman M: Development of occlusion; University of Pennsylvania press Philadelphia 1941

Gurdsapsri W, Ai M, Baba K, Fueki K (2000): Influence of clenching level on intercuspal contact area in various regions of the dental arch. J Oral Rehabil 27, 239-244

Halperin GC, Halperin AR, Norling BK (1982): Thickness, strength, and plastic deformation of occlusal registration strips. J Prosthet Dent $\underline{48}, 575-578$

Haralur S, Al-Gadhaan S, Al-Qahtani A, Mossa A, Al-Shehri W, Addas M (2014): Influence of functional head postures on the dynamic functional occlusal parameters. Ann Med Health Sci Res $\underline{4}, 562-566$

Harper KA, Setchell DJ (2002): The use of shimstock to assess occlusal contacts: a laboratory study. Int J Prosthodont 15, 347-352 
Harvey WL, Hatch RA, Osborne JW (1991): Computerized occlusal analysis. J Prosthet Dent $\underline{65}, 89-92$

Hidaka O, Iwasaki M, Saito M, Morimoto T (1999): Influence of clenching intensity on bite force balance, occlusal contact area, and average bite pressure. J Dent Res $\underline{78}, 1336-1344$

Hugger A, Türp JC, Kerschbaum T: Curriculum orale Physiologie; Quintessenz Verl. Berlin 2006

Hützen D, Proff P, Gedrange T, Biffar R, Bernhard O, Kocher T, Kordass B (2007): Occlusal contact patterns -- population-based data. Ann Anat 189, 407-411

Hützen D, Rebau M, Kordass B (2006): Clinical reproducibility of GEDAS--"Greifswald Digital Analyzing System" for displaying occlusal contact patterns. Int J Comput Dent $\underline{9}, 137-142$

Imamura Y, Sato Y, Kitagawa N, Uchida K, Osawa T, Omori M, Okada Y (2015): Influence of occlusal loading force on occlusal contacts in natural dentition. J Prosthodont Res $\underline{59}, 113-120$

Iwase M, Ohashi M, Tachibana H, Toyoshima T, Nagumo M (2006): Bite force, occlusal contact area and masticatory efficiency before and after orthognathic surgical correction of mandibular prognathism. Int J Oral Maxillofac Surg $\underline{35}$, 1102-1107

Jankelson B (1979): Neuromuscular aspects of occlusion. Effects of occlusal position on the physiology and dysfunction of the mandibular musculature. Dent Clin North Am 23, 157-168

Kalachev YS, Michailov TA, Iordanov PI (2001): Study of occlusal-articulation relationships with the help of T-SCAN apparatus. Folia Med $\underline{43}, 88-91$

Katayama N, Koide K, Koide K, Mizuhashi F (2018): The influence of horizontal cephalic rotation on the deviation of mandibular position. J Adv Prosthodont 10, 401-407

Keith JF (2017): The Glossary of Prosthodontic Terms: Ninth Edition. J Prosthet Dent $\underline{94}$, 1105

Kerstein RB (1993): A comparison of traditional occlusal equilibration and immediate complete anterior guidance development. Cranio $\underline{11}, 126-140$

Kerstein RB (2001): Current applications of computerized occlusal analysis in dental medicine. Gen Dent $\underline{49}, 521-530$

Kerstein RB (2004): Combining technologies: a computerized occlusal analysis system synchronized with a computerized electromyography system. Cranio 22, 96-109

Kerstein RB (2008a): Articulating paper mark misconceptions and computerized occlusal analysis technology. Dent Implantol Update $\underline{19}, 41-46$

Kerstein RB (2008b): Computerized occlusal analysis technology and Cerec case finishing. Int J Comput Dent 11, 51-63

Kerstein RB (2008c): T-scan III applications in mixed arch and complete arch, implant -supported prosthodontics. Dent Implantol Update 19, 49-53

Kerstein RB (2010): Reducing chronic masseter and temporalis muscular hyperactivity with computer-guided occlusal adjustments. Compend Contin Educ Dent $\underline{31}$, 530-538 
Kerstein RB, Grundset K (2001): Obtaining measurable bilateral simultaneous occlusal contacts with computer-analyzed and guided occlusal adjustments. Int J Oral Maxillofac Implants $\underline{32}$, 718

Kerstein RB, Lowe M, Harty M, Radke J (2006): A force reproduction analysis of two recording sensors of a computerized occlusal analysis system. Cranio $\underline{24}, 15-24$

Kerstein RB, Radke J (2012): Masseter and temporalis excursive hyperactivity decreased by measured anterior guidance development. Cranio $\underline{30}$, 243-254

Kerstein RB, Wilkerson DW (2001): Locating the centric relation prematurity with a computerized occlusal analysis system. Compend Contin Educ Dent 22, 525-536

Kerstein RB, Wright NR (1991): Electromyographic and computer analyses of patients suffering from chronic myofascial pain-dysfunction syndrome: before and after treatment with immediate complete anterior guidance development. J Prosthet Dent 66, 677-686

Kijak E, Margielewicz J, Lietz-Kijak D, Wilemska-Kucharzewska K, Kucharzewski M, Śliwiński Z (2017): Model identification of stomatognathic muscle system activity during mastication. Exp Ther Med 13, 135-145

Kinoshita S, Taguchi S, Fukuda M (1982): A new method for occlusal examination and record by using Occlusal Prescale (R) (author's transl). Shikai Tenbo 모, 311-320

Kirveskari P, Alanen P, Jämsä T (1989): Association between craniomandibular disorders and occlusal interferences. J Prosthet Dent 62, 66-69

Koeck B, Engelhardt JP, Haunfelder D, Diedrich P, Horch HH (Hrsg.): Funktionsstörungen des Kauorgans, 3. Aufl; Urban \& Schwarzenberg München 1995

Koos B, Godt A, Schille C, Goz G (2010): Precision of an instrumentation-based method of analyzing occlusion and its resulting distribution of forces in the dental arch. J Orofac Orthop 71, 403-410

Koos B, Holler J, Schille C, Godt A (2012): Time-dependent analysis and representation of force distribution and occlusion contact in the masticatory cycle. J Orofac Orthop $\underline{73}$, 204-214

Kordaß B, Lovrov S (2013): Methods for evaluation of occlusal contacts. J CranioMand Func $\underline{5}, 247-264$

Korioth TW (1990): Number and location of occlusal contacts in intercuspal position. J Prosthet Dent $\underline{64}, 206-210$

Kumagai H, Suzuki T, Hamada T, Sondang P, Fujitani M, Nikawa H (1999): Occlusal force distribution on the dental arch during various levels of clenching. J Oral Rehabil 26, 932-935

Lentner E, Rammelsberg P, Böhm A, Pospiech A, Gernet W (1997): Zum Untersuchereinfluss auf Lage und Reproduzierbarkeit der zentrischen Kondylenposition. Dtsch Zahnarztl Z 06, $411-415$

Lucia VO (1962): The gnathological concept of articulation. Dent Clin North Am $\underline{6}, 183$

Lyons MF, Sharkey SW, Lamey PJ (1992a): An evaluation of the T-Scan computerised occlusal analysis system. Int J Prosthodont $\underline{2}, 166-172$ 
Lyons MF, Sharkey SW, Lamey PJ (1992b): An evaluation of the T-Scan computerised occlusal analysis system. Int J Prosthodont $\underline{5}, 166-172$

Mahony D (2005): Refining occlusion with muscle balance to enhance long-term orthodontic stability. J Clin Pediatr Dent 29, 93-98

Malta Barbosa J, Brian Urtula A, Hirata R, Caramês J (2018): Thickness evaluation of articulating papers and foils. J Esthet Restor Dent $\underline{30}, 70-72$

Maness WL, Benjamin M, Podoloff R, Bobick A, Golden RF (1987): Computerized occlusal analysis. Quintessenz J 18, 287-292

Maness WL, Podoloff R (1989): Distribution of occlusal contacts in maximum intercuspation. J Prosthet Dent $\underline{62}, 238-242$

Manns A, Chan C, Miralles R (1987): Influence of group function and canine guidance on electromyographic activity of elevator muscles. J Prosthet Dent $\underline{57}$, 494-501

Manns A, Miralles R, Valdivia J, Bull R (1989): Influence of variation in anteroposterior occlusal contacts on electromyographic activity. J Prosthet Dent 61, 617-623

Matsui Y, Ohno K, Michi K, Suzuki Y, Yamagata K (1996): A computerized method for evaluating balance of occlusal load. J Oral Rehabil 23, 530-535

McDevitt WE, Warreth AA (1997): Occlusal contacts in maximum intercuspation in normal dentitions. J Oral Rehabil 24, 725-734

McNamara DC, Henry PJ (1974): Terminal hinge contact in dentitions. J Prosthet Dent $\underline{32}$, 405-411

Michalakis KX, Pissiotis A, Anastasiadou V, Kapari D (2004): An experimental study on particular physical properties of several interocclusal recording media. Part II: Linear dimensional change and accompanying weight change. J Prosthodont $\underline{13}$, 150-159

Millstein PL (1983): An evaluation of occlusal contact marking indicators. Quintessenz J $\underline{14}$, 813-836

Millstein PL (1984): A method to determine occlusal contact and noncontact areas. J Prosthet Dent $\underline{52}, 106-110$

Millstein PL (1985): An evaluation of occlusal indicator wax. J Prosthet Dent $\underline{53}, 570-572$

Millstein PL (2008): Know your indicator. J Mass Dent Soc 므, 30-31

Millstein PL, Clark RE, Kronman JH (1973): Determination of the accuracy of wax interocclusal registrations. Part II. J Prosthet Dent 29, $40-45$

Miralles R, Manns A, Pasini C (1988): Influence of different centric functions on electromyographic activity of elevator muscles. Cranio $\underline{6}, 26-33$

Miyazaki H, Motegi E, Yatabe K, Yamaguchi H, Maki Y (2005): A study of occlusion in elderly Japanese over 80 years with at least 20 teeth. Gerodontology 22, 206-210 
Murray MC, Smith PW, Watts DC, Wilson NF (1999): Occlusal registration:. Int Dent J $\underline{49}$, 4146

Myers GE, Anderson JR (1971): Nature of contacts in centric occlusion in 32 adults. J Dent Res $\underline{50}, 7-13$

Nadershah M (2019): Prevalence of temporomandibular joint disorders in adults in Jeddah, Kingdom of Saudi Arabia: A cross-sectional study. J Contemp Dent Pract 20, 1009-1013

Namarig SH KN (2015): The relationship of lateral excursive contact schemes with occlusal tooth wear among medical campus students in University of Khartoum. J Dent Oral Health $\underline{1}$, $1-6$

Nayar S, Babu R (2007): Occlusion indicators. J Indian Prosthodont Soc $\underline{7}, 170$

Nelson SJ: Wheeler's dental anatomy, physiology, and occlusion, 10. Auflage; Elsevier Saunders St. Louis, Mo. 2015

Nishimori H, Iida T, Kamiyama H, Komoda Y, Obara R, Uchida T, Kawara M, Komiyama O (2017): Comparing the occlusal contact area of individual teeth during low-level clenching. J Oral Sci $\underline{59}, 337-342$

Obara R, Komiyama O, Iida T, Asano T, Laat A de, Kawara M (2014): Influence of different narrative instructions to record the occlusal contact with silicone registration materials. J Oral Rehabil 41, 218-225

Obara R, Komiyama O, Iida T, Laat A de, Kawara M (2013): Influence of the thickness of silicone registration material as a means for occlusal contact examination--an explorative study with different tooth clenching intensities. J Oral Rehabil $\underline{40}, 834-843$

Ogawa T, Ogimoto T, Koyano K (1998): Pattern of occlusal contacts in lateral positions. J Prosthet Dent $\underline{80}, 67-74$

Ogawa T, Ogimoto T, Koyano K (2001): The relationship between non-working-side occlusal contacts and mandibular position. J Oral Rehabil 28, 976-981

Ohmure H, Miyawaki S, Nagata J, Ikeda K, Yamasaki K, Al-Kalaly A (2008): Influence of forward head posture on condylar position. J Oral Rehabil $\underline{35}$, 795-800

Okeson JP (1995): Occlusion and functional disorders of the masticatory system. Dent Clin North Am $\underline{39}$, 285-300

Okeson JP: Management of temporomandibular disorders and occlusion, 8. Auflage; Mosby St. Louis 2019

Ottl P, Ahlers MO, Lange M, Utz KH, Reiber T (2018): Untersuchungsbogen Klinischer Funktionsstatus. ZWR 127, 227-236

Palaskar JN, Murali R, Bansal S (2013): Centric relation definition. J Indian Prosthodont Soc $\underline{13}$, 149-154

Panek H, Matthews-Brzozowska T, Nowakowska D, Panek B, Bielicki G, Makacewicz S, Mankiewicz M (2008): Dynamic occlusions in natural permanent dentition. Quintessence Int 39, 337-342 
Parker MW (1993): The significance of occlusion in restorative dentistry. Dent Clin North Am 37, 341-351

Pasricha N, Sidana V, Bhasin S, Makkar M (2012): Canine protected occlusion. Indian J Oral Sci $\underline{3}, 13$

Patel M, Alani A (2015): Clinical issues in occlusion - Part II. Singapore Dent J $\underline{36}, 2-11$

Patyk A, Lotzmann U, Paula JM, Kobes LW (1989a): Ist das T-Scan-System eine diagnostisch relevante Methode zur Okklusionskontrolle? ZWR 98, 686, 688, 693-94

Patyk A, Lotzmann U, Scherer C, Kobes LW (1989b): Vergleichende okklusionsanalytische Studie zur klinischen Anwendbarkeit des T-Scan Systems. ZWR 98, 752-755

Plasmans PJ, van Eil FA, Vrijhoef MM, van't Hof MA (1988): The occlusal topographic contact method for the assessment of occlusal contacts. J Dent $\underline{16}, 18-21$

Posselt U (1958): Range of movement of the mandible. J Am Dent Assoc 56, 10-13

Preiskel HW (1965): Some observations on the postural position of the mandible. J Prosthet Dent $\underline{15}, 625-633$

Qadeer S: The limitations of traditional non-digital occlusal indicators when compared to the T-Scan computerized occlusal analysis technology. In: Management Association IR (Hrsg.): The limitations of traditional non-digital occlusal indicators when compared to the T-Scan computerized occlusal analysis technology: IGI Global. Hershey, Pennsylvania, USA 2017, 1528-1555

Qadeer S, Kerstein R, Kim RJ, Huh J-B, Shin S-W (2012): Relationship between articulation paper mark size and percentage of force measured with computerized occlusal analysis. J Adv Prosthodont $\underline{4}, 7-12$

Ramfjord SP, Ash MM: Occlusion; W.B. Saunders Philadelphia 1967

Rateitschak KH, Wolf F (Hrsg.): Funktionsdiagnostik und Therapieprinzipien, 8. Auflage; Thieme Stuttgart 2015

Reiber T, Fuhr K, Hartmann H, Leicher D (1989): Das Zeichnungsverhalten von Okklusionsindikatoren. I. Einfluss der Indikatorstärke, des Druckes und der Oberflächenmorphologie. Dtsch Zahnarztl Z 44, 90-93

Reiber T, Müller F (1994): Klinische Untersuchungen zur statischen Okklusion. Dtsch Zahnarztl Z $\underline{49}, 363-366$

Ricketts RM (1950): Variations of the temporomandibular joint as revealed by cephalometric laminagraphy. Am J Orthod $\underline{36}, 877-898$

Ricketts RM (1969): Occlusion—the medium of dentistry. J Prosthet Dent 21, 39-60

Riise C (1982): A clinical study of the number of occlusal tooth contacts in the intercuspal position at light and hard pressure in adults. J Oral Rehabil $\underline{9}$, 469-477

Riise C, Ericsson SG (1983): A clinical study of the distribution of occlusal tooth contacts in the intercuspal position at light and hard pressure in adults. J Oral Rehabil $\underline{10}, 473-480$ 
Riise C, Sheikholeslam A (1984): Influence of experimental interfering occlusal contacts on the activity of the anterior temporal and masseter muscles during mastication. J Oral Rehabil 11 , $325-333$

Rinchuse DJ, Kandasamy S, Sciote J (2007): A contemporary and evidence-based view of canine protected occlusion. Am J Orthod Dentofacial Orthop 132, 90-102

Sander FM, Schwenzer N, Ehrenfeld M (Hrsg.): Kieferorthopädie, 2. Auflage; Thieme München 2011

Saraçoğlu A, Ozpinar B (2002): In vivo and in vitro evaluation of occlusal indicator sensitivity. J Prosthet Dent $\underline{88}, 522-526$

Schelb E, Kaiser DA, Brukl CE (1985): Thickness and marking characteristics of occlusal registration strips. J Prosthet Dent $\underline{54}, 122-126$

Schmitter M, Rammelsberg P, Hugger A, Freesmeyer WB, Morneburg T, Türp JC, Utz KH (2010): Wissenschaftliche Mitteilung der Deutschen Gesellschaft für Prothetische Zahnmedizin und Biomaterialien e.V. (vormals DGZPW): Kieferrelationsbestimmung. Dtsch Zahnarztl Z, 2010

Scholz W, Pancherz H, Reichel R (1991): Beurteilung des T-SCAN-Systems zur Registrierung der okklusalen Kontaktverhältnisse. Zahnärztl Prax, 6-9

Schuyler CH (1929): Principles employed in full denture prostheses which may be applied to other fields of dentistry. J Am Dent Assoc 16, 2045-2054

Sessle BJ (2005): Biological adaptation and normative values. Int J Prosthodont $\underline{18}$, 280-282

Setz J, Geis-Gerstorfer J (1990a): Messeigenschaften eines Systems zur digitalen Okklusionsdiagnostik. Dtsch Zahnarztl Z, 65-66

Setz J, Geis-Gerstorfer J (1990b): Messeigenschaften eines Systems zur digitalen Okklusionsdiagnostik. Dtsch Zahnarztl Z, 65-66

Sharma A, Rahul GR, Poduval ST, Shetty K, Gupta B, Rajora V (2013): History of materials used for recording static and dynamic occlusal contact marks. J Clin Exp Dent $\underline{5}, 48-53$

Shupe RJ, Mohamed SE, Christensen LV, Finger IM, Weinberg R (1984): Effects of occlusal guidance on jaw muscle activity. J Prosthet Dent $\underline{51}, 811-818$

Sidana V, Bhasin S, Makkar M, Pasricha N (2012): Group function occlusion. Indian J Oral Sci $\underline{3}, 124$

Singh A, Sangur R, Rao BL, Mahajan T (2013): A clinical study to determine the pattern of occlusal contacts in lateral positions and its validity in classifying guidance patterns. J Indian Prosthodont Soc 13, 101-107

Singh BP (1970): Centric occlusion and centric relation (retruded) positions of the mandible. J Indian Dent Assoc 42, 131-134

Sodeyama A, Shinogaya T, Matsumoto M (1998): Reproducibility of maximal bite force distribution over dentition. Kokubyo Gakkai zasshi 65, 339-343 
Solberg WK (1986): Temporomandibular disorders. Br Dent J 160, 317-322

Spee F (1890): Die Verschiebungsbahn des Unterkiefers am Schädel. Archiv für Anatomie und Entwicklungsgeschichte, 285-294

Sreekumar AV, Rupesh PL, Pradeep N (2012): Nature of occlusion during eccentric mandibular movements in young adults. J Contemp Dent Pract $\underline{13}, 612-617$

Stallard H, Stuart C (1963): Concepts of occlusion. Dent Clin North Am 7, 591-600

Strub JR, Kern M, Türp JC, Witkowski S, Heydecke G, Wolfart S (Hrsg.): Artikulatoren, Ästhetik, Werkstoffkunde, festsitzende Prothetik, 4. Auflage; Quintessenz-Verl. Berlin 2011a

Strub JR, Kern M, Türp JC, Witkowski S, Heydecke G, Wolfart S (Hrsg.): Geschichte, Grundlagen, Behandlungskonzept, Vorbehandlung, 4. Auflage; Quintessenz-Verl. Berlin 2011b

Suzuki T, Kumagai H, Watanabe T, Uchida T, Nagao M (1997): Evaluation of complete denture occlusal contacts using pressure-sensitive sheets. Int J Prosthodont 10, 386-391

Suzuki T, Kumagai H, Yoshitomi N, Minakuchi S, Watanabe T, Uchida T, Ishinabe S, Sekita T, Kobayashi K (1994): Clinical evaluation of measuring system of occlusal force. Kokubyo Gakkai zasshi $\underline{61}$, 437-445

Takai A, Nakano M, Bando E, Hewlett ER (1993): Evaluation of three occlusal examination methods used to record tooth contacts in lateral excursive movements. J Prosthet Dent $\underline{70}, 500$ 505

Takai A, Nakano M, Bando E, Hewlett ER (1995): Influence of occlusal force and mandibular position on tooth contacts in lateral excursive movements. J Prosthet Dent $\underline{73}, 44-48$

Tejo SK, Kumar AG, Kattimani VS, Desai PD, Nalla S, Chaitanya K K (2012): A comparative evaluation of dimensional stability of three types of interocclusal recording materials-an in-vitro multi-centre study. Head Face Med $\underline{8}, 27$

Throckmorton GS, Rasmussen J, Caloss R (2009): Calibration of 'T-Scan sensors for recording bite forces in denture patients. J Oral Rehabil $\underline{36}$, 636-643

Thumati P (2015): Clinical outcome of subjective symptoms in myofascial pain patients treated by immediate complete anterior guidance development technique using digital analysis of occlusion (Tek-scan) and electromyography. J Interdiscip Dentistry $\underline{5}, 12$

Tschernitschek H, Handel G, Gunay H (1990): Möglichkeiten und Grenzen eines neuen okklusionsdiagnostischen Verfahrens. Zahnärztl Prax, 54-56

Türp JC, Greene CS, Strub JR (2008): Dental occlusion. J Oral Rehabil 35, 446-453

Tzakis MG, Karlsson S, Carlsson GE (1992): Effects of intense chewing on some parameters of masticatory function. J Prosthet Dent $\underline{67}$, 405-409

Utz KH: Die taktile Feinsensibilität natürlicher Zähne: Eine klinisch-experimentelle Untersuchung. Me. Diss. Bonn 1982 
Utz KH, Duvenbeck H, Oettershagen K (1990): Distanz zwischen terminaler Scharnierachsenposition und Interkuspidationsposition im Kiefergelenkbereich. SSO Schweiz Monatsschr Zahnheilkd 100, 42-49

van't Spijker A, Creugers NH, Bronkhorst EM, Kreulen CM (2011): Body position and occlusal contacts in lateral excursions. Int J Prosthodont 24, 133-136

Visscher CM, Huddleston Slater JJ, Lobbezoo F, Naeije M (2000): Kinematics of the human mandible for different head postures. J Oral Rehabil 27, 299-305

Walder DN (1969): Claudication. Bristol Med Chir J ㅁ4, 153-157

Walter M, Tomomatsu M (1990): Beurteilungen des T-Scan-Systems zur Registrierung der okklusalen Kontaktverhältnisse. ZWR, 966-968

Wang YL, Cheng J, Chen YM, Yip KH, Smales RJ, Yin XM (2011): Patterns and forces of occlusal contacts during lateral excursions recorded by the T-Scan II system in young Chinese adults with normal occlusions. J Oral Rehabil $\underline{38}, 571-578$

Weber T (Hrsg.): Zahnmedizin; Georg Thieme Verlag Stuttgart 2016

Wieckiewicz M, Grychowska N, Zietek M, Wieckiewicz W (2016): Evaluation of the Elastic Properties of Thirteen Silicone Interocclusal Recording Materials. Biomed Res Int 2016, 1-8

Wiegelmann S, Bernhardt O, Meyer G (2015): The associations between occlusal parameters in static and dynamic occlusion and the signs and symptoms of temporomandibular disorders. J CranioMand Func 7, 27-38

Williamson EH, Lundquist DO (1983): Anterior guidance: its effect on electromyographic activity of the temporal and masseter muscles. J Prosthet Dent $\underline{49}$, 816-823

Wiskott HA, Belser UC (1995): A rationale for a simplified occlusal design in restorative dentistry. J Prosthet Dent $\underline{73}, 169-183$

Woda A, Gourdon AM, Faraj M (1987): Occlusal contacts and tooth wear. J Prosthet Dent $\underline{57}$, 85-93

Woda A, Vigneron P, Kay D (1979): Nonfunctional and functional occlusal contacts: a review of the literature. J Prosthet Dent $\underline{42}, 335-341$

Yamamura M, Takahashi A, Aoki H, Takeuchi N, Endo Y, Tamaki K, Sano Y, Fujiwara S, Fujita T, Kusunoki S (1990): A study on display and accuracy of occlusal contacts by means of T-Scan System. Kanagawa Shigaku 25, 236-241

Yamamura Y, Kurachi M, Yokoya R, Murata T, Nonogaki R (2005): Relationship of the movement of mandibular condyle and occlusal force balance. Nihon Hotetsu Shika Gakkai Zasshi $\underline{49}, 56-64$

Yemm R (1969): Variations in the electrical activity of the human masseter muscle occurring in association with emotional stress. Arch Oral Biol 14, 873-878

Yu A, Lee H (2010): A wax guide to measure the amount of occlusal reduction during tooth preparation in fixed prosthodontics. J Prosthet Dent $\underline{103}$, 256-257 
Ziebert GJ, Donegan SJ (1979): Tooth contacts and stability before and after occlusal adjustment. J Prosthet Dent $\underline{42}$, 276-281 


\section{Danksagung}

Herzlich danke ich meinem Doktorvater Herrn Prof. Dr. Ralf Bürgers für die intensive Begleitung im Rahmen meines Forschungsprojektes und die Möglichkeit in der Poliklinik für Zahnärztliche Prothetik promovieren zu dürfen. Herrn Prof. Dr. Philipp Meyer-Marcotty danke ich für die Ko-Betreuung und Anleitung während des gesamten Studienprozesses. Dankbar darf ich auf ein exzellentes Arbeitsklima bei hervorragender wissenschaftlicher Begleitung zurückblicken.

Für die geduldige und engagierte Erstellung der statistischen Modelle zur Unterstützung meiner wissenschaftlichen Erkenntnisse danke ich Herrn Dr. Andreas Leha aus dem Institut für Medizinische Statistik der Universitätsmedizin Göttingen. 